\title{
Cross-Layer Media Access Control in Wireless Body Area Networks Using Fuzzy Logic and Evolutionary Computation
}

\author{
by \\ Seyed Mohammad Nekooei
}

\author{
A thesis \\ submitted to the Victoria University of Wellington \\ in fulfilment of the \\ requirements for the degree of \\ Doctor of Philosophy \\ in Computer Science.
}

Victoria University of Wellington 2017 



\begin{abstract}
Over the past decade, advances in electronics, computer science, and wireless technologies have facilitated the rapid development of Wireless Body Area Networks (WBANs). WBANs consist of various sensors that are attached on or even implanted in the human body to improve health care and the quality of life. WBANs must provide high-quality communication in terms of both reliability and performance, in order to bring timely medical help to patients. Commonly used communication standard in WBANs is IEEE 802.15.4. However, due to poor channel quality in WBANs, this standard is limited in reliability and performance. To address this issue, cross-layer techniques for Media Access Control (MAC) have attracted substantial research attention in recent years.

Aimed at developing cross-layer MAC technologies, Fuzzy Logic Controllers (FLCs) have been widely utilised to effectively and efficiently process information from different layers in WBANs. However, existing FLCs have mostly focused on improving communication reliability while ignoring the importance of network performance.

To improve both the reliability and performance of MAC protocols in WBANs, this thesis introduces a new design of cross-layer FLC, called Cross-Layer Fuzzy logic based Backoff system (CLFB), to improve reliability by reducing the collision rate and increasing the packet delivery ratio. CLFB can also enhance the network performance in terms of throughput in WBANs while maintaining packet delays at a reasonable level by considering both channel condition and application requirements. Through the proper use of FLCs as an extension of the standard exponential backoff algorithms, CLFB is experimentally shown to achieve a high level of adaptability.
\end{abstract}


This thesis also proposes an evolutionary approach to automate the design of FLCs for CLFB in WBANs. With the goal of improving network reliability while keeping the communication delay at a low level, we have particularly studied the usefulness of three coding schemes with different levels of flexibility, which enables us to represent alternative design of FLCs as candidate solutions in evolutionary algorithms. The influence of fitness functions that measure the effectiveness of each possible FLC design has also been examined carefully in order to identify useful FLCs. Moreover, we have utilised surrogate models to improve the efficiency of the design process. In consideration of practical usefulness, we have further identified two main design targets. The first target is to design effective FLCs for a specific network configuration. The second target covers a wide range of network settings. In order to examine the usefulness of our design approach, we have utilised and experimentally evaluated two popularly used evolutionary algorithms, i.e. Particle Swarm Optimisation (PSO) and Differential Evolution (DE).

This thesis finally proposes a two-level control scheme at both the sensor level and the coordinator level to further improve communication quality in WBANs. The sensor-level FLC controls contention based channel access and the coordinator-level FLC controls contention free channel access. This two-level FLC architecture can effectively enhance the cooperation between sensors and the coordinator such that both the reliability and performance of the network can be significantly improved. We also studied the use of cooperative coevolutionary approach to automate the design of our twolevel control scheme. With the goal of effectively designing useful FLCs, we have particularly investigated different collaborator selection methods for our cooperative coevolutionary approach, which enable us to effectively select collaborators while evaluating the candidate FLC design in each sub-population. Specifically, we show that network knowledge can help our evolutionary design approach to select collaborators more effectively. 


\section{Dedication}

To my parents for their endless support. 


\section{Acknowledgements}

This thesis has only been possible with the support of numerous people. Firstly, I would like to express my sincere gratitude to my supervisors Dr Aaron Chen and Dr Ramesh Rayudu for the continuous support of my Ph.D study and related research. Specially, I am grateful to Dr Aaron Chen for his patience, motivation, and immense knowledge.

Besides my supervisors, I would like to thank the rest of my thesis committee: Prof. Brijesh Verma, Dr Ian Welch, and Dr Xuejun Li, for letting my defence be an enjoyable moment, and their insightful comments and encouragement.

I thank Prof Dale Carnegie, Dr Peter Andreae, Dr Will Browne, Mrs Patricia Stein and Mrs Suzan Hall for their kindness and support through all these years. I would also like to thank all of student Learning Support, specially Dr. Deborah Laurs and Mrs. Madeleine Collinge, who supported me in writing this thesis. I also thank to those unnamed, but essential at Victoria University of Wellington.

I would especially like to thank NeSI high-performance computing facilities and their technical support to the results of my Ph.D. thesis.

Last but not the least, I would like to thank my family and friends for supporting me spiritually throughout writing this thesis and my life in general.

Seyed Mohammad Nekooei

Victoria University of Wellington 


\section{List of publications}

- Seyed Mohammad Nekooei, Gang Chen and Ramesh Kumar Rayudu, "A fuzzy logic based cross-layer mechanism for medium access control in WBAN," 2015 IEEE 26th Annual International Symposium on Personal, Indoor, and Mobile Radio Communications (PIMRC), Hong Kong, 2015, Pages 1094-1099.

- Seyed Mohammad Nekooei, Gang Chen and Ramesh Kumar Rayudu, "Evolutionary design of fuzzy logic controllers for medium access control in WBAN," 2016 IEEE Congress on Evolutionary Computation (CEC), Vancouver, BC, 2016, Pages 2821-2828.

- Seyed Mohammad Nekooei, Gang Chen and Ramesh Kumar Rayudu, "Automatic Design of Fuzzy Logic Controllers for Medium Access Control in Wireless Body Area Networks - an Evolutionary Approach," Applied Soft Computing, Volume 56, 2017, Pages 245-261.

- Seyed Mohammad Nekooei, Gang Chen and Ramesh Kumar Rayudu, "Cooperative Design of Two Level Fuzzy Logic Controllers for Medium Access Control in Wireless Body Area Networks," 11th International Conference on Simulated Evolution and Learning (SEAL2017), Shenzhen, China, 2017. 


\section{Contents}

1 Introduction $\quad 1$

1.1 Problem Statement . . . . . . . . . . . . . . . 1

1.2 Motivation . . . . . . . . . . . . . . . . 6

1.3 Research Goals . . . . . . . . . . . . . . . . . . . 9 9

1.3.1 Research Questions . . . . . . . . . . . . . . 99

1.3.2 Research Objectives ... . . . . . . . . . . . . . 11

1.3.3 Major Contributions ... . . . . . . . . . . . . . 13

1.4 Organisation of the Thesis . . . . . . . . . . 16

2 Technical Background and Literature Review 19

2.1 Technical Background ... . . . . . . . . . . . . . . . . 19

2.1.1 Wireless Body Area Networks (WBANs) . . . . . . . 20

2.1.2 Medium Access Control (MAC) . . . . . . . . . . 22

2.1 .3 IEEE $802.15 .4 \ldots \ldots \ldots \ldots$

2.1.4 Fuzzy Logic Controller (FLC) . . . . . . . . . . . . 33

2.1.5 Evolutionary Computation (EC) . . . . . . . . . . 39

2.1.6 Differential Evolution (DE) . . . . . . . . . . . . 42

2.1.7 Particle Swarm Optimization (PSO) . . . . . . . . . . 43

2.1.8 Cooperative Particle Swarm Optimisation (CPSO) . . 44

2.2 Literature review . . . . . . . . . . . . . . . . . . . 46

2.2.1 Medium Access Control (MAC) . . . . . . . . . 46

2.2.2 Cross-layer Fuzzy Logic MAC . . . . . . . . . . . . 51

2.2.3 Evolutionary design of Fuzzy Logic Controllers . . . 53 
2.3 Chapter Summary . . . . . . . . . . . . . . . . . . 60

3 Cross-Layer Fuzzy Logic based Backoff 63

3.1 Introduction . . . . . . . . . . . . . . . 63

3.1 .1 Chapter Goals . . . . . . . . . . . . . . . 65

3.1 .2 Chapter Organisation . . . . . . . . . . 65

3.2 A Cross-Layer Backoff Mechanism Using Fuzzy Logic Controller . . . . . . . . . . . . . . . . 66

3.2.1 Fuzzy Input and Output Variables . . . . . . . . . . 69

3.2.2 Fuzzy Logic Rules . . . . . . . . . . . . . . . . 71

3.3 Manual Design of Fuzzy Logic Controllers . . . . . . . . . . 72

3.4 Simulation Implementation and Results . . . . . . . . . . . 74

3.4.1 Simulation Environment . . . . . . . . . . . 76

3.4 .2 Simulation Results . . . . . . . . . . . . . 77

3.5 Chapter Summary . . . . . . . . . . . . . . . . . . 81

4 Automatic Design of Fuzzy Logic Controller in Wireless Body Area Networks $\quad 85$

4.1 Introduction . . . . . . . . . . . . . 85

4.1 .1 Chapter Goals . . . . . . . . . . . . . . 87

4.1.2 Chapter Organisation . . . . . . . . . . . . . 89

4.2 Evolutionary Design of FLCs in WBANs . . . . . . . . . . . 89

4.2.1 Coding Scheme . . . . . . . . . . . . . . 89

4.2 .2 Fitness Function . . . . . . . . . . . . . 93

4.2.3 Fitness Evaluation Method . . . . . . . . . . . . 96

4.2.4 Surrogate Assisted ECs for Designing FLCs . . . . . . 97

4.3 Simulation Implementation and Results . . . . . . . . . . 100

4.3.1 Simulation Environment for WBANs . . . . . . . 101

4.3.2 Parameter Settings for Evolutionary Algorithms . . . 102

4.3 .3 Evaluation Metrics . . . . . . . . . . . . . . . . . 102

4.3.4 Automatic Design of FLCs for Specific Network Settings $\left(\right.$ Target $\mathrm{T}_{1}$ ) . . . . . . . . . . . . . . . 104 
4.3.5 Automatic Design of FLCs for Multiple Network Settings $\left(\right.$ Target $\left.\mathrm{T}_{2}\right) \ldots \ldots \ldots \ldots \ldots \ldots$

4.4 Chapter Summary . . . . . . . . . . . . . . . . . . . . . . . 124

5 Cooperative Design of Two Level Fuzzy Logic Controllers for Medium Access Control

5.1 Introduction . . . . . . . . . . . . . . . . . . . . 127

5.1.1 Chapter Goals . . . . . . . . . . . . . . . . . . . . . 130

5.1.2 Chapter Organisation . . . . . . . . . . . . . . . 130

5.2 Cross-Layer GTS Allocation using FLC . . . . . . . . . . . . 131

5.2.1 Fuzzy Input and Output Variables . . . . . . . . . . 133

5.2.2 Fuzzy Logic Rules . . . . . . . . . . . . . . . . . . . 135

5.3 Evolutionary Design of Multilevel FLCs . . . . . . . . . . 136

5.3.1 Multilevel FLC Design based on CPSO . . . . . . . 138

5.3.2 Collaborator Selection Method . . . . . . . . . . . . 140

5.3.3 Fitness Evaluation Method . . . . . . . . . . . . . . 146

5.4 Simulation Implementation and Results . . . . . . . . . . 146

5.4.1 Simulation Environment for WBAN . . . . . . . . . . 147

5.4.2 Parameter Settings for Evolutionary Algorithms . . . 147

5.4.3 Evaluation Metrics . . . . . . . . . . . . . . . . . . . 148

5.4.4 Automatic Design of Multilevel FLCs for Specific Network Settings (Target $\mathrm{T}_{1}$ ) _ . . . . . . . . 148

5.4.5 Automatic Design of Multilevel FLCs for Multiple Network Settings $\left(\right.$ Target $\mathrm{T}_{2}$ ) _ . . . . . . . . 152

5.5 Chapter Summary . . . . . . . . . . . . . . . . . . . . . . . 157

6 Conclusions 163

6.1 Achieved Research Objectives . . . . . . . . . . . . . . . . . 164

6.2 Main Conclusions . . . . . . . . . . . . . . . . . . . . . . . . 166

6.2.1 Cross-Layer Fuzzy Logic based Backoff . . . . . . . . 166

6.2.2 Automatic Design of Fuzzy Logic Controllers . . . 167 
6.2.3 Cooperative Design of Two Level Fuzzy Logic Controllers . . . . . . . . . . . . . . 170

6.3 Future Works . . . . . . . . . . . . . . . . . . . 171

6.3 .1 IEEE $802.15 .6 \ldots \ldots \ldots \ldots 17 \ldots \ldots \ldots$

6.3.2 Multi-Objective EC Techniques . . . . . . . . . . . 171

6.3.3 Surrogate-assisted Cooperative Coevolution . . . . . 172

6.3.4 Real Life Experiments . . . . . . . . . . . . . . 173

6.4 Final Remarks . . . . . . . . . . . . . . . . . . . . . . . 173

$\begin{array}{lr}\text { A High performance Computation } & 199\end{array}$ 


\section{List of Figures}

1.1 Wireless Body Area Network ... . . . . . . . . . 2

2.1 Examples of WBAN topologies . . . . . . . . . . . . 23

2.2 A sending an RTS to the coordinator and the coordinator responding with a CTS. . . . . . . . . . . . . . . . 25

2.3 IEEE 802.15.4 frame structure . . . . . . . . . . . . . . . 27

2.4 Example of the IEEE 80215.4 superframe structure . . . . . . 28

2.5 Slotted CSMA/CA algorithm . . . . . . . . . . . . . . 29

2.6 IEEE 802.15.4 GTS allocation . . . . . . . . . . . . . . . . 32

2.7 Basic diagram of the Fuzzy Logic Control System . . . . . . 37

2.8 State transition scheme to adjust macMinBE in IEEE 802.15.4, proposed by Ko et al. [51] . . . . . . . . . . . . . . . 48

3.1 The flow chart of CSMA/CA and CLFB algorithms where differences between the standard CSMA/CA and CrossLayer Fuzzy logic Backoff (CLFB) are highlighted. . . . . . . 67

3.2 Example of Wireless Body Area Network which uses IEEE 802.15.4 modified by Cross-Layer Fuzzy Logic based Backoff (CLFB) controller . . . . . . . . . . . . . . . 68

3.3 Architecture of fuzzy logic controller for CLFB. . . . . . . . . 69

3.4 An example of triangular membership functions and tuning by adjusting the membership function parameters . . . . . 71

3.5 The tuned membership functions . . . . . . . . . . . 75 
4.1 The design process of CLFB, where an evolutionary process adjusts the rule base obtained by an expert and the initial data base. . . . . . . . . . . . . . . . . 90 90

4.2 Unrestricted coding scheme (URCS) in which $T$ is the linguistic of each membership function. . . . . . . . . . . . 91

4.3 Strictly restricted coding scheme (SRCS) in which $T$ is the linguistic of each membership function. . . . . . . . . . 92

4.4 Moderately restricted coding scheme (MRCS) in which $T$ is the linguistic of each membership function. $k$ is the number of overlapping control factors (OCFs) which are represented

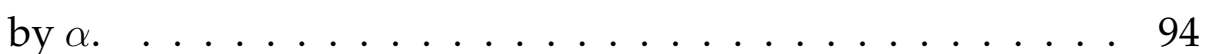

4.5 An example of surrogate model to calculate the fitness function. 99

4.6 An example of surrogate model to calculate the fitness function.100

4.7 The PSO-based process for FLC design will converge after 100 generations. The results for all coding schemes are obtained by averaging over 30 independent tests. . . . . . . . 103

4.8 Example of designed FLC through UnRestricted Coding Scheme $($ URCS) . . . . . . . . . . . . . . . . . 108

4.9 Example of designed FLC through Strictly Restricted Coding Scheme $($ SRCS) . . . . . . . . . . . . . . . 108

4.10 Example of designed FLC through Moderately Restricted Coding Scheme (MRCS) . . . . . . . . . . . . . . . . . 109

5.1 Architecture of Cooperative Cross-Layer Fuzzy Medium Access Control (CoCLF-MAC) which includes Cross-Layer Fuzzy logic Backoff (CLFB) at the sensor level and CrossLayer Fuzzy logic GTS Allocation (CLFGA) at the coordinator level . . . . . . . . . . . . . . . . . . 132

5.2 Architecture of fuzzy logic controller for CLFGA. . . . . . . 134

5.3 Coevolutionary model of two populations . . . . . . . . . . . 140

5.4 An example of similarity measurements . . . . . . . . . . . 142 
5.5 The CPSO-based process for FLC design after 100 generations. The results for all collaborator selection methods are obtained by averaging over 30 independent tests in a four-sensor-node scenario. . . . . . . . . . . . . . . . . . 151 


\section{List of Tables}

2.1 Related works of EA-based FLCs design. . . . . . . . . . . 55

3.1 Fuzzy Logic rules for CLFB. . . . . . . . . . . . . . . . 73

3.2 Communication specifications of Sensor nodes used in the simulation $[2,119] . \ldots \ldots \ldots \ldots$

3.3 Packet Delivery Ratio (PDR) from the average of 30 independent runs, the improvement of CLFB over IEEE 802.15.4 is highlighted . . . . . . . . . . . . . . 80

3.4 Collision rate from the average of 30 independent runs, the improvement of CLFB over IEEE 802.15.4 is highlighted . . . 80

3.5 MAC Throughput in bit per seconds from the average of 30 independent runs, the improvement of CLFB over other algorithms is highlighted . . . . . . . . . . . 82

3.6 Packet end-to-end delay in seconds from the average of 30 independent runs, the improvement of CLFB over IEEE 802.15 .4 is highlighted . . . . . . . . . . . . . . . 82

4.1 Communication specifications of Sensor nodes used in the simulation $[2,119] . \ldots \ldots \ldots$

4.2 Results obtained by PSO in terms of PDR and packet delay in seconds $\left(\right.$ target $\left.T_{1}\right) \ldots \ldots \ldots$. . . . . . . . 106

4.3 Results obtained by DE in terms of PDR and packet delay (seconds). . . . . . . . . . . . . . . . . . 111

xvii 
4.4 Results obtained by surrogate model for PSO and DE in terms of PDR and packet delay (seconds). . . . . . . . . . 113

4.5 Average computation time of PSO and DE with our HPC facilities over 30 independent runs. . . . . . . . . . . . . . . 114

4.6 Results obtained by competing algorithms, i.e. IEEE 802.15.4, $\mathrm{D}^{2} \mathrm{MAC}$ and NB-step. . . . . . . . . . . . . . . 115

4.7 WBAN settings for designing FLCs regarding target $\mathrm{T}_{2}$. . . 117

4.8 WBAN settings for testing FLCs designed regarding target $\mathrm{T}_{2} \ldots \ldots \ldots \ldots \ldots \ldots \ldots 117$

4.9 Training results obtained by PSO and surrogate-assisted PSO in terms of PDR and packet delay in seconds $(\lambda=2.5) . \quad \ldots 119$

4.10 Results obtained by competing algorithms, i.e. IEEE 802.15.4, $\mathrm{D}^{2} \mathrm{MAC}$ and NB-step, in term of PDR. . . . . . . . . . . 121

4.11 Results obtained by competing algorithms, i.e. IEEE 802.15.4, $\mathrm{D}^{2} \mathrm{MAC}$ and NB-step, in term of collision rate. . . . . . . . 122

4.12 Results obtained by competing algorithms, i.e. IEEE 802.15.4, $\mathrm{D}^{2} \mathrm{MAC}$ and NB-step, in term of throughput (bps). . . . . . . 123

4.13 Results obtained competing algorithms, i.e. IEEE 802.15.4, $\mathrm{D}^{2} \mathrm{MAC}$ and NB-step, in term of delay (seconds). . . . . . . 125

5.1 Fuzzy Logic rules for CLFGA. . . . . . . . . . . . . . . . 136

5.2 Collaborator selection criteria for sensor-level FLC design . . 143

5.3 Collaborator selection criteria for coordinator-level FLC design143

5.4 Communication specifications of Sensor nodes used in the simulation $[2,119] . \ldots \ldots 149$

5.5 Comparison of CoCLF-MAC ${ }_{T_{1}}$ and competing algorithms, in terms of PDR and packet delay (seconds), throughput (bps) and Collision rate. . . . . . . . . . . . . . . . . 152

5.6 WBAN settings for designing Multilevel FLCs regarding target $\mathrm{T}_{2} \ldots \ldots \ldots \ldots \ldots \ldots \ldots \ldots$

5.7 WBAN settings for testing Multilevel FLCs designed regarding target $\mathrm{T}_{2} \ldots \ldots \ldots \ldots \ldots \ldots \ldots \ldots$ 
5.8 Training results obtained by CPSO in terms of PDR and packet delay in seconds $(\lambda=2.5) \ldots \ldots$. . . . . . . . 155

5.9 Results obtained by competing algorithms, i.e. IEEE 802.15.4, AGA+ IEEE 802.15.4, AGA+CLFB and FCMA, in term of PDR.158

5.10 Results obtained by competing algorithms, i.e. IEEE 802.15.4, AGA+ IEEE 802.15.4, AGA+CLFB and FCMA, in term of collision rate. . . . . . . . . . . . . . . . . 159

5.11 Results obtained by competing algorithms, i.e. IEEE 802.15.4, AGA+ IEEE 802.15.4, AGA+CLFB and FCMA, in term of throughput (bit/s). . . . . . . . . . . . . . . . . 160

5.12 Results obtained by competing algorithms, i.e. IEEE 802.15.4, AGA+ IEEE 802.15.4, AGA+CLFB and FCMA, in term of delay (seconds). . . . . . . . . . . . . . . . . 161

A.1 The specifications of High Performance Computing (HPC) platforms . . . . . . . . . . . . . . . . 199 


\section{List of Acronyms and Abbreviations}

$\begin{array}{ll}\text { Abbreviation } & \text { Description } \\ \text { ACS } & \text { Additional Carrier Sensing } \\ \text { AGA } & \text { Adaptive GTS Allocation } \\ \text { ANOVA } & \text { Analysis of Variance } \\ \text { BE } & \text { Backoff Exponent } \\ \text { BP } & \text { Backoff Period } \\ \text { CAP } & \text { Contention Access Period } \\ \text { CC } & \text { Cooperative Coevolution } \\ \text { CCA } & \text { Clear Channel Assessment } \\ \text { CCEA } & \text { Cooperative Co-Evolutionary Algorithms } \\ \text { CCGA } & \text { Cooperative Coevolutionary Genetic Algorithm } \\ \text { CCPSO } & \text { Cooperative Coevolving Particle Swarm Optimization } \\ \text { CFP } & \text { Contention Free Period } \\ \text { CLFB } & \text { Cross-Layer Fuzzy logic based Backoff } \\ \text { CLFGA } & \text { Cross-Layer Fuzzy logic based GTS Allocation } \\ \text { CoCLF-MAC } & \text { Cooperative Cross Layer Fuzzy MAC } \\ \text { CPSO } & \text { Cooperative Particle Swarm Optimization } \\ \text { Cr } & \text { Crossover Rate } \\ \text { CSMA/CA } & \text { Carrier Sense Multiple Access with Collision Avoidance } \\ \text { CW } & \text { Contention Window }\end{array}$




$\begin{array}{ll}\text { Abbreviation } & \text { Description } \\ \text { D }{ }^{2} \text { AC } & \text { Dynamic delayed Medium Access Control } \\ \text { DACE } & \text { Design and Analysis of Computer Experiments } \\ \text { DE } & \text { Differential Evolutionary } \\ \text { EA } & \text { Evolutionary Algorithm } \\ \text { EB } & \text { Enhanced Backoff } \\ \text { ECR } & \text { Enhanced Collision Resolution } \\ \text { EC } & \text { Evolutionary Computation } \\ \text { ECG } & \text { Electrocardiogram } \\ \text { ECR } & \text { Enhanced Collision Resolution } \\ \text { EEG } & \text { Electroencephalogram } \\ \text { EMO } & \text { Evolutionary Multi-Objective } \\ \text { EP } & \text { Evolutionary Programming } \\ \text { ES } & \text { Evolutionary Strategy } \\ \text { FCFS } & \text { First-Come-First-Served } \\ \text { FCMA } & \text { Fuzzy Control Medium Access } \\ \text { FFD } & \text { Full Function Devices } \\ \text { FLC } & \text { Fuzzy Logic Controller } \\ \text { FSM } & \text { Finite-State Machine } \\ \text { GA } & \text { Genetic Algorithm } \\ \text { GP } & \text { Genetic programming } \\ \text { GSA } & \text { GTS Scheduling Algorithm } \\ \text { GTS } & \text { Guaranteed Time Slot } \\ \text { i-GAME } & \text { Implicit GTS Allocation MEchanism } \\ \text { LOS } & \text { Line-Of-Sight } \\ \text { MAC } & \text { Media Access Control } \\ \text { MBWA } & \text { Mobile Broadband Wireless Access } \\ \text { MF } & \text { Membership Function } \\ \text { MLP } & \text { Multi-Layer Perceptron } \\ \text { MRCS } & \text { Moderately Restricted Coding Scheme } \\ \text { NB } & \text { Number of Backoffs } \\ \text { NFC } & \text { Neuro-Fuzzy Control } \\ & \end{array}$




\begin{tabular}{ll} 
Abbreviation & Description \\
NiCS & Network Inspired Collaborator Selection \\
NLOS & Non-Line-Of-Sight \\
OCF & Overlapping Control Factor \\
PDR & Packet Delivery Ratio \\
PHY & Physical layer \\
PID & Proportional-Integral-Derivative \\
PSO & Particle Swarm Optimization \\
RFD & Reduced Function Devices \\
RBF & Radial Basis Function \\
SI & Swarm Intelligence \\
SpO2 & Peripheral capillary Oxygen Saturation \\
SRCS & Strictly Restricted Coding Scheme \\
SD & Superframe Duration \\
URCS & UnRestricted Coding Scheme \\
WBAN & Wireless Body Area Network \\
WMAN & Wireless Metropolitan Area Network \\
WPAN & Wireless Personal Area Networks \\
WSAN & Wireless Sensor and Actuator Network \\
WSN & Wireless Sensor Network \\
& \\
\hline &
\end{tabular}




\section{List of Symbols}

\begin{tabular}{|c|c|}
\hline Symbols & Description \\
\hline $\overrightarrow{\vec{X}}$ & Candidate solution vector \\
\hline$F$ & Scaling factor \\
\hline pbest & Personal best \\
\hline gbest & Global best \\
\hline$\vec{V}$ & Velocity vector \\
\hline$P_{j} . x_{i}$ & $\begin{array}{l}\text { Current position of the } i \text { th candidate solution of } \\
\text { the } j \text { th swarm }\end{array}$ \\
\hline$P_{j} \cdot$ pbest $_{i}$ & $\begin{array}{l}\text { Personal best of the } i \text { th candidate solution of the } \\
j \text { th swarm }\end{array}$ \\
\hline$P_{j} . g b e s t$ & Global best of $j$ th swarm \\
\hline$\lambda$ & $\begin{array}{l}\text { Coefficient to control balance PDR and packet delay } \\
\text { in the fitness function }\end{array}$ \\
\hline$\delta$ & Discount factor in moving average \\
\hline$\eta$ & Number of controlled individuals \\
\hline$P_{j} \cdot \tilde{\mathrm{y}}$ & the selected collaborator in the $j$ th sub-population \\
\hline
\end{tabular}




\section{Chapter 1}

\section{Introduction}

This chapter provides an introduction to this thesis and its motivations, goals, contributions, and organisation. The problem statement is provided first, followed by a discussion on the motivations and challenges. The research goals and major contributions of this thesis are then discussed. This chapter finally presents the thesis organisation.

\subsection{Problem Statement}

A Wireless Body Area Network (WBAN) is an emerging technology which deploys intelligent, low-power sensor nodes on, around or even implanted in, the human body. As shown in Figure 1.1, each WBAN will have one special node that is the coordinator, or the hub. This could be a smart phone or another more powerful device, which receives bio-information from the sensors and actuators. Often the coordinator is equipped with a larger battery. It provides an interface with other networks or central motioning station such as medical staff. In other words, the WBAN architecture can be divided into two levels: sensor level and coordinator level.

WBAN connects the patient to the external medical health system in order to transfer the vital signs of the patient to medical staff working in the hospital. WBAN has the potential to make medical equipment movement 


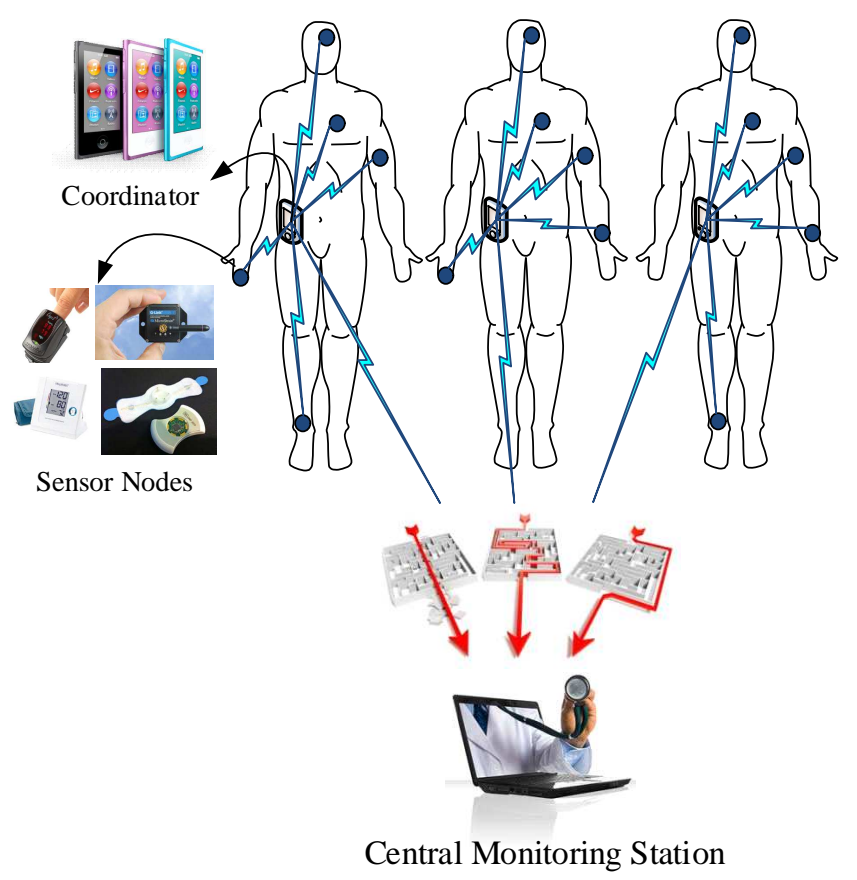

Figure 1.1: Wireless Body Area Network

easier where medical devices can be positioned without any worry about re-establishing the patient's connection to medical devices by reducing the reliance on conventional cords and cables. In addition, it provides more freedom in real-time monitoring especially for ambulatory patients within the hospital, and home or mobile-health monitoring situations. It has a great potential for monitoring chronically ill patients continuously over long periods. Moreover, comprehensive real-time patient information is available for mobile staff and server-based intelligent software algorithms to monitor patients and make initial decisions. For example, WBAN can monitor the heartbeat using Electrocardiography (ECG) sensors on an ongoing basis. Then, the information will go from sensors to a coordinator for transmitting the information to a point to integrate data. This would create more realistic observations for medical staff to analyse.

The WBAN must provide high-quality communication support, i.e. reliability and performance, in order to bring timely medical help to a 
patient. In practice, reliability is an important requirement, because WBAN is responsible for carrying critical medical data and signalling emergencies such as vital organ failures. In case of an emergency, communication failure could cause death when a life-threatening event has been left unattended. It must also provide high performance communication to support sensors with high transmission rate, such as ECG sensors. Consequently, as a critical component for intelligent health and medical monitoring, one of the major concerns of the WBAN is to meet the performance and reliability requirements of various WBAN applications, specially in healthcare.

In WBANs, Media Access Control (MAC) plays an important role for providing reliability and performance [169]. WBANs, similar to other wireless networks, must share a communication channel among multiple nodes to transmit information. Therefore, WBANs utilise a mechanism called MAC protocol to control channel (or medium) access. Since MAC protocols control the channel access among nodes, their efficiency and effectiveness can affect the reliability and performance of the network through determining when a node can access the channel and resolving any potential conflicts among competing nodes.

IEEE 802.15 family of wireless communication standards, in particular IEEE 802.15.4, outline the basic elements needed to provide interoperability among different devices [139] and defined MAC protocols for low power devices in WBANs [3, 2]. However, they do not consider a complete MAC protocol for WBANs. For example, they provide a packet format and relevant message exchange protocols including allocation interval assignment and the procedure to acknowledge the reception of packets. But, they do not support communication control policies in consideration of application requirements, channel condition, prioritising and retransmission $[4,3]$. Moreover, past research has evaluated the efficiency of IEEE 802.15.4, showing that due to poor channel quality in WBANs, this standard can be very limited in terms of its reliability (measured by Packet Delivery Ratio (PDR)), and performance (measured by throughput and packet delay) 
$[152,120,53,24,113,25,54]$. Therefore, these MAC protocols need to be improved to cope with this poor channel and stay reliable. It means when the channel is available it should be used efficiently.

Several researchers $[151,91]$ have tried to tackle these challenges by considering WBANs as a special type of Wireless Sensor Network (WSN) or Wireless Sensor and Actuator Network (WSAN). Nevertheless, the provided solutions for these types of networks cannot solve the challenges associated with monitoring of human bodies specifically [151,91]. In terms of human bodies, we face a complex interaction between the internal and external environment of the body. Whenever wireless sensor networks are used to monitor human health and behaviours, these sensors may need to be attached to or implanted in the body tissues. In essence, in addition to different challenges faced by WSNs, the human body environment is different with respect to scale, type, and frequency of monitoring. In a WBAN, limited number of nodes are deployed in, on or around a single person in a small-scale area, which is smaller than general WSNs. Another important difference is communication reliability between each WBAN node, which is overcome by redundancy in WSN. The redundancy could not be a solution for WBANs, because, for each typical medical application of WBANs, there is usually a single sensor for each vital sign. Homogeneous sensors networks are major assumptions for most WSN protocols, but are not applicable in heterogeneous WBAN.

To cope with these challenges, MAC protocols can exploit information from other layers to efficiently use the current channel capacity. For example, channel access can be scheduled based on channel condition. Particularly, when the channel quality is good, MAC protocol should efficiently utilise the channel. Meanwhile, when channel becomes available, MAC protocol should prioritise channel access based on the known application data rate. Specifically, MAC protocol can increase the network reliability and performance by allowing high data rate sensor nodes to access the channel with higher priorities and improve the utilisation of the channel 
capacity by sending as many packets as possible. This design technique is called cross layering which has attracted much research attention in WBANs because of the possible performance gain. Cross-layering is expected to effectively deal with multiple sources of information uncertainty and make complicated decisions in an efficient and effective manner. Therefore, joint cooperation across layers demands complex algorithms.

Soft computing techniques, in particular fuzzy logic, have been popularly used in cross-layering systems [120, 107, 31]. Existing studies have also clearly shown that fuzzy logic is particularly useful for cross-layer control of channel access because of its flexibility and simplicity [120, 107, 31]. Due to its ability to handle uncertainty and imprecision, fuzzy logic has also been characterised as one of the most effective technologies for successfully controlling sophisticated processes. It enables the use of simple controllers to satisfy complex design requirements, particularly when the model of the system to be controlled has uncertainties [13]. This approach is particularly suitable for dealing with nonlinear systems, such as WBANs, with multiple inputs of a diverse nature, defining rules to connect these inputs with the output.

However, FLCs have not been used effectively in WBANs (refer to Section 1.2). For example, researchers often focus on designing FLC to improve only one WBAN's requirement (e.g. reliability) rather than balancing between different requirements (e.g. reliability and performance) $[107,177,31]$. Moreover, researchers in the literature have mostly used FLCs at sensor level only, neglecting the importance of using FLCs at the coordinator level to improve the quality of communication [107, 115, 31]. Furthermore, although it is possible to improve MAC protocols by using FLCs, high-quality communication in WBANs relies heavily on carefully designed FLCs and poses new challenges for research. [120, 31, 107, 113]. 


\subsection{Motivation}

The research in this thesis is motived by two requirements: (1) network requirements and (2) design requirements. To address the first requirement, FLCs have been incorporated into cross-layer based MAC protocols. Many researchers have specifically used FLCs to process cross-layer information and improve the reliability or performance of IEEE 802.15.4 [115, 120, 107, 31]. Instead of tweaking parameters of existing communication standard, they substituted FLCs for part of the communication standard [107, 177, 31] in hope of improving communication quality which is often measured based on a single application requirement. For example, they could manage to achieve high reliability by sacrificing communication delays $[115,107,31]$. Furthermore, existing works have considered mainly one level of channel access control [107, 115, 31]. Particularly, cross-layer FLCs have often been applied to the sensor level control and the opportunity of multilevel FLCs control in WBANs is frequently ignored [107, 115, 31]. However. it has been shown that IEEE 802.15.4 is able to improve its reliability and performance when both sensor and coordinator level control of media access can effectively work together [135, 64].

The second requirement is closely associated with the fact that, in practice, the effectiveness of FLCs depends heavily on their control rules and Membership Functions (MFs). Therefore, careful design of FLCs becomes an important research issue. However, existing research works have not addressed this issue in depth [120, 31, 107, 113, 108].

Motivated by the two requirements above, we summarise the challenges we have investigated in this thesis below:

- In practice, reliability is an important requirement. Based on this understanding, some existing research works have been conducted to enhance the level of reliability $[119,107,31]$. However, most of these works failed to use used FLCs in an effective manner. For example, instead of using FLCs to tune the parameters of the exponential back- 
off algorithm and in response to changing channel conditions and application requirements, most researchers [107, 177, 31] focused on utilising FLCs to increase backoff delays for the purpose of improving the reliability and performance at the cost of significantly increasing the packet delay [113]. However, it is very important to note that, the average packet delay must be kept at a reasonably low level in time-critical WBAN applications. Driven by this understanding, aimed at achieving a desirable balance between reliability and performance and supporting application requirements, a new approach must be developed in this thesis to enable independent use of FLCs by multiple sensor nodes for distributed cross-layer MAC.

- Designing multi-level MAC based on cross-layer FLCs is a challenging task in WBANs. Many researchers have utilised FLCs for sensor level (i.e distributed) MAC to improve the communication reliability and performance $[107,115]$. Meanwhile, it has been shown that the coordinator level control (i.e centralised) can also play an important role to improve the reliability and performance of the network [135, 64]. In fact, some research efforts have been performed to optimise the coordinator-level resource allocation, in particular bandwidth allocation, among sensor nodes. Nevertheless, to the best of our knowledge, few research efforts have exploited the possibilities of using both distributed and centralised communication methods. Therefore, it is of particular significance to develop an effective multi-level crosslayer FLC system that simultaneously supports both sensor level and coordinator level MAC in IEEE 802.15.4.

- Although FLCs have potential to improve the communication quality, it has been shown that their effectiveness highly depends on their design. Building an effective FLC involves designing and tuning several parameters such as the position, shape and distribution of MFs, rule base construction, selection of logical operations and conse- 
quences of the rules. Obviously, careful design of FLCs is essential for successful communications in WBANs [120, 31, 107, 113]. However, a review of the literature suggests that this important design issue in WBANs has not been fully addressed. Very often, researchers rely purely on manual design of FLCs that requires huge human efforts, yet cannot provide any performance guarantees [120, 107, 31, 113]. Various automatic techniques have also been utilised to design FLCs $[159,168,96,23,52,59,47]$. Among these techniques, Evolutionary Computation (EC) techniques enjoy a clear advantage since they are capable of automatically designing different types of fuzzy logic rules, various forms of MFs, and the structure of the rules base [45]. Meanwhile, two FLC design requirements should be satisfied: (1) effectiveness and (2) interpretability. The former refers to the capability of FLCs to provide high reliability and performance without having a negative impact on packet delays in WBANs. The latter requires the behaviour of WBAN MAC protocols to be expressed in an easily understandable way.

- Designing two FLCs, which can be cooperatively used at both the sensor level and coordinator level, is also challenging, as there are large number of parameters to be designed and tuned. Clearly, manual approaches are not desirable because they are laborious and error-prone. Conventional EC techniques are not desirable either, because when the dimension of each candidate solution increases, conventional EC techniques may quickly lose their effectiveness [143]. Therefore, utilising cooperative coevolutionary computation techniques could be a promising and suitable solution to design FLCs effectively and cooperatively. However, one major challenge of using cooperative coevolutionary computation techniques is the choice of collaborators while evaluating the candidate FLC design in each sub-population. Many research works recommend to choose the best fit or random solution from alternative sub-populations [126, 156, 161]. However, 
these methods are designed to tackle general optimisation problems. It remains as an important and open question to develop a new collaborator selection method that can perform more effectively for our specific design problems.

\subsection{Research Goals}

The overall goal of this thesis is to study fuzzy logic based cross-layer approaches to improve reliability and performance of MAC protocols in WBANs. For this purpose, this research aims to investigate the use of cross-layer based FLCs to improve the reliability and performance of IEEE 802.15.4 based MAC protocols. This research also aims to investigate EC techniques for automatic and effective design of FLCs for cross-layer MAC in WBANs.

\subsubsection{Research Questions}

The research in this thesis will help to answer the research questions below:

(i) How can cross-layer FLCs be effectively used by distributed sensor nodes to improve both reliability and performance of WBANs compared to existing cutting-edge algorithms?

Reliability and performance are essential, yet challenging, requirements in WBANs. The cross-layer approach is a promising approach to address this issue by sharing information across multiple communication layers. The use of FLCs for cross-layer MACs in WBANs is motivated by their flexibility and simplicity. However, current state-of-the-art research focuses on either reliability or performance, therefore has limited practical usefulness. Consequently, we have the goal to design an effective cross-layer FLC for distributed MAC among sensor nodes in WBANs to improve both communication reliability and performance. 
(ii) How can cross-layer FLCs be employed both at the sensor level and the coordinator level to control channel access and significantly improve the reliability and performance of WBANs?

Both contention based (i.e distributed) and contention free (i.e. centralised) methods must be supported for effective MAC in WBANs. Although each method is often studied individually, the reliability and performance of WBANs can be further improved by using both of them cooperatively and simultaneously. However, few research works have ever investigated such two-level design of cross-layer FLCs in WBANs where the first level focuses on controlling contention based channel access and the second level controls contention free channel access. The key challenge is how to balance these two cross-layer FLC levels to significantly improve both reliability and performance in WBANs compared to other cutting-edge algorithms.

(iii) How can EC techniques be applied to automatically design effective and interpretable FLCs for sensor nodes to control channel access in the context of WBANs?

In practice careful design of FLCs can significantly improve their effectiveness. EC techniques have been widely shown to be highly effective at automatically designing various of fuzzy logic rules, Membership Functions (MFs), and the structure of rules base [45]. In this thesis, EC techniques will be developed to design FLCs to provide desirable balance between reliability and performance in WBANs. Due to high computation complexity of EC techniques, the overall efficiency of the design process must be significantly enhanced.

(iv) How can EC techniques be applied to automatically design effective two-level FLCs both at the sensor and the coordinator level to cooperatively control channel access in WBANs?

To answer research question (ii), a two-level cross-layer FLC system will be developed where FLCs at the sensor level must collaborate 
effectively with the FLC at the coordinator level. This requires joint design of two FLCs, one for sensor nodes to control contention based channel access and another one for the coordinator to control contention free channel access. This design problem motivates the use of Cooperative Coevolutionary Algorithms (CCEAs) to reduce search spaces and improve design effectiveness. While CCEA has shown definite promise on various problems, it still faces challenging research questions. One major question is regarding choice of collaborators based on problem characteristics.

\subsubsection{Research Objectives}

The following set of research objectives have been defined in order to fulfil the overall goal and research questions.

1. Propose a distributed cross-layer FLC architecture for IEEE 802.15.4 based MAC protocols to enhance both reliability and performance of WBANs.

This objective aims to propose a new cross-layer approach using FLCs to control contention based channel access. In the literature, most of the existing research works on using FLCs in WBANs focus on improving either the communication reliability or performance $[113,107,31]$. However, they mainly focus on either reliability or performance [113, 107, 31]. Different from these works, this research aims to provide a desirable balance between reliability and performance by introducing a distributed FLC architecture for cross-layer MAC. This goal will be achieved by enhancing the performance of a standard exponential backoff algorithm by effectively utilising cross-layer information including channel condition and application requirements. This objective will be explored in Chapter 3 .

2. Propose an automatic approach for effectively and efficiently designing FLCs using EC techniques in the context of WBANs. 
Despite the promising results of using FLCs, careful design of FLCs is required for effective MAC in WBANs. However, existing research works mostly rely on a manual approach to design FLC, which is laborious and error-prone. Despite huge human efforts, the resulting FLC may have poor quality in practice. To address this problem, this research proposes an evolutionary approach for automated design of FLCs. In addition to improving network reliability and performance, this research also aims to apply surrogate models to improve the efficiency and practicality of the design approach. This objective will be covered in Chapter 4.

3. Propose a two-level cross-layer FLC scheme for IEEE 802.15.4 based MAC protocols to take advantages of both distributed and centralised mechanisms, i.e at both sensor level and coordinator level, to cooperatively enhance reliability and performance of WBANs.

Following the first objective, which proposes to use a distributed cross-layer FLC architecture for enhancing contention based channel access, this objective aims at proposing a two-level cross-layer FLC architecture to improve the reliability and performance of WBANs further by exploiting both distributed and centralised mechanisms. The first cross-layer FLC will control the contention based channel access at the sensor level and the second one will control contention free channel access at the coordinator level. This two level FLC architecture is expected to effectively enhance the cooperation between sensors and the coordinator such that both the reliability and performance of the network can be significantly improved.

To improve the effectiveness of our multilevel FLCs, through this objective we aim to adopt a Cooperative Co-evolutionary approach to automatically design multi-level FLCs. This research also aims to propose a new network-inspired collaborator selection mechanism by exploiting the specific characteristics of the design problem. This 
objective will be addressed in Chapter 5 .

A summary of the major contributions of this thesis is presented in Section 1.3.3.

\subsubsection{Major Contributions}

This section presents the major contribution of this thesis, whereas each of the Chapters 3 to 5 is dedicated to in-depth discussions of respective contributions.

1. This thesis introduces a cross-layer FLC for IEEE 802.14.4 based MACs, called Cross-Layer Fuzzy logic based Backoff system (CLFB), to improve both network reliability (measured by PDR) and performance (measured by throughput and packet delays). Contrary to existing algorithms, which increase either reliability or performance, the new approach explicitly considers both of the essential requirements. In particular, based on channel condition and application requirements, CLFB can improve reliability and performance without increasing packet delay, therefore achieving a higher level of adaptability in comparison to IEEE 802.15.4. We also present a manual approach to design the fuzzy membership functions in CLFB in order to enhance its effectiveness. By integrating our CLFB into the IEEE 802.15.4 MAC sub-layer, we successfully enhance the competence of this IEEE standard for various WBAN-based applications. Moreover, backward compatibility is ensured since this integration does not change the underlying structure of IEEE 802.15.4. The results clearly show that our CLFB achieved noticeable improvement over several cutting-edge algorithms in terms of both network reliability and performance.

Parts of this contribution have been published in:

Seyed Mohammad Nekooei, Gang Chen and Ramesh Kumar Rayudu, "A fuzzy logic based cross-layer mechanism for medium access control 
in WBAN," In IEEE 26th Annual International Symposium on Personal, Indoor, and Mobile Radio Communications (PIMRC), (Aug 2015), pp. 1094-1099.

2. This thesis introduces a new EA-based approach for fully automated design of FLCs for CLFB in WBANs. To ensure the practicality of our approach, we have defined three major requirements: effectiveness, efficiency and general applicability. (1) Effectiveness: We have demonstrated the effectiveness of our approach through experimental results. We have successfully shown that our approach can design FLCs effectively to provide both reliability and performance in WBANs. Particularly, we have compared CLFB using our automated designed FLCs to other state-of-art algorithms. The comparison shows that FLCs design through our approach can outperform other competing algorithms in terms of both reliability and performance. (2) Efficiency: In this research, the computation time of designing FLCs is used to examine the efficiency of the approach. We have enhanced the efficiency of our approach by using surrogate models. We have experimentally shown that our automatic design approach based on surrogate models has significantly improved the efficiency of the design process. (3) General applicability: We have introduced two design targets. The first target is to improve communication quality for patients who have a specific WBAN configuration. The second target focuses on MAC under multiple WBAN configurations. We found that the designed rules can provide a reasonably good balance between PDR and delay for a wide range of WBAN settings. That means the effectiveness of designed FLCs can be preserved with respect to different network settings.

Parts of this contribution have been published in:

Seyed Mohammad Nekooei, Gang Chen and Ramesh Kumar Rayudu, "Evolutionary design of fuzzy logic controllers for medium access 
control in WBAN," In IEEE Congress on Evolutionary Computation (IEEE CEC) (July 2016), pp. 2821-2828.

Seyed Mohammad Nekooei, Gang Chen, Ramesh Kumar Rayudu, "Automatic design of fuzzy logic controllers for medium access control in wireless body area networks - An evolutionary approach", Applied Soft Computing 56 (2017), 245-261.

3. This thesis introduces Cooperative Cross Layer Fuzzy MAC controller (CoCLF-MAC) which employs a two-level cross-layer FLC, i.e. CrossLayer Fuzzy logic based GTS Allocation controller (CLFGA) and CLFB, to improve reliability and performance of the IEEE 802.15.4-based MAC in the context of WBANs. Specifically, while CLFB is utilised at sensor level, CLFGA at the coordinator level employs FLCs for prioritising GTS allocation by using cross-layer information, including channel condition and application data rate. In contrast to many related algorithms, which use cross-layer optimisation only at the sensor level, the new approach uses cross-layer FLC at both sensor level and coordinator level, to cooperatively schedule the channel access and improve communication quality.

This thesis also introduces a CCEA-approach for fully automated design of FLCs at both the sensor level and coordinator level. In order to improve effectiveness of the design approach, we introduced two collaborator selection methods that employ the network knowledge to judge the suitability of potential collaborator designs. We found that our new collaborator selection methods can improve the effectiveness of the design process. We investigated the practicality of our approach based on two aspects: effectiveness and general applicability. (1) Effectiveness: We have shown experimentally that, based on FLCs automatically designed by CCEAs, our CoCLF-MAC achieved significant improvement over other cutting-edge algorithms in terms of both network reliability and performance. (2) General applicability: 
We have designed our CoCLF-MAC based on two design targets. The first target is to improve communication quality based on a specific WBAN configuration. The second target aims to improve communication quality over multiple WBAN configurations. Both targets have been achieved successfully, clearly demonstrating the practical usefulness of our approach.

Parts of this contribution have been published in:

Seyed Mohammad Nekooei, Gang Chen and Ramesh Kumar Rayudu, “ Cooperative Design of Two Level Fuzzy Logic Controllers for Medium Access Control in Wireless Body Area Networks," 11th International Conference on Simulated Evolution and Learning (SEAL2017), Shenzhen, China, 2017.

\subsection{Organisation of the Thesis}

This thesis is divided into six chapters followed by the Appendix and References section.

Chapter 1 presents an introduction to the thesis. It provides an overview of the research field, thesis objectives, its original contribution to scientific knowledge, and thesis organisation.

Chapter 2 presents background knowledge and literature review.

Chapter 3 presents Cross-Layer Fuzzy logic based Backoff system (CLFB). In this chapter, we present the usefulness of utilising FLCs for crosslayer MAC in WBANs. We also study FLCs design to improve reliability and performance of WBANs.

Chapter 4 studies the use of evolutionary algorithms (EAs) to fully automate the design of FLCs for cross-layer medium access control in WBANs. It studies three coding schemes and their usefulness during the proposed evolutionary design process. It also examines the 
influence of fitness functions that measure the effectiveness of each possible FLC design in order to achieve a good balance between network reliability and performance. Moreover, we utilise surrogate models to improve the efficiency of the design process.

Chapter 5 presents a new two-levelled MAC scheme called Cooperative Cross Layer Fuzzy MAC (CoCLF-MAC). CoCLF-MAC is designed to support effective MAC at both the sensor level and the coordinator level in WBANs. This chapter also presents comprehensive investigations over the use of cooperative coevolutionary algorithms to fully automate the design of our two-level control scheme. With the goal of improving network reliability while keeping the communication delay at a low level, this chapter studies and experimentally evaluates five different collaborator selection methods for our design problem.

Chapter 6 presents conclusions and discussion of results and limitations. Future research directions are also highlighted. 


\section{Chapter 2}

\section{Technical Background and Literature Review}

This chapter provides technical background and related works for this thesis. This chapter in Section 2.1 covers important background and provides definitions of the basic concepts and terminologies in Wireless Body Area Networks (WBANs) such as Media Access Control (MAC) protocols and IEEE 802.15.4 standard. Section 2.1 also provides a brief introduction to Fuzzy Logic Controls (FLCs) and Evolutionary Computation (EC) techniques. Then in Section 2.2, we review related work and summary the research topics related to WBANs. Section 2.2 also reviews related work to FLC design problems. Finally, this chapter in Section 2.3 presents a summary of challenges.

\subsection{Technical Background}

This section first covers basic concepts of WBANs in Subsection 2.1.1. Subsection 2.1.2 presents technical background of MACs in WBANs. Subsection 2.1.3 gives general overview on IEEE 802.15.4. Then Subsection 2.1.4 briefly presents FLCs. Subsections 2.1 .5 covers EC techniques in general. Finally Subsection 2.1.6, 2.1.7 and 2.1.8 cover detailed EC techniques used in 
this thesis which are Differential Evolution (DE), Particle Swarm Optimisation (PSO) and Cooperative PSO (CPSO) respectively.

\subsubsection{Wireless Body Area Networks (WBANs)}

\section{Applications}

Today, most images of organs, medical tests, and information about patients are hours or even days old by the time doctors see them. However, in hospitals facilitated by WBANs, physicians can check images, medical tests, and medical history in real time even as they operate. In addition, wireless actuators in WBANs deliver drugs more accurately. Accurate drug delivery can lower doses and reduce side effects, such as a spinal cord stimulator implanted in the body for long-term pain relief [89].

When the patient becomes unconscious, the patients' WBAN will automatically identify the coordinates, alert the authorities, and upload their entire medical history through an external medical health system before nurses and doctors arrive. WBANs also can be used to help patients, such as asthmatics who are allergic to some environmental factors. For example, Chu et al. [33] utilised WBANs to monitor environmental factors. In an environment which the asthmatic patient is allergic, an alarm is triggered.

As healthcare costs increase and the world population age [153], there is also a need to monitor a patient's health status while he/she is out of the hospital in his/her personal environment. To address this demand, WBANs can be used to monitor patients' health. This also gives patients as much autonomy and even responsibility for themselves and their environment as possible [141, 121]. Computer-assisted rehabilitation and therapy can also become accessible for patients at their homes with the help of WBANs [162].

WBANs can also serve in other widespread domains. For example, athletes training for the Olympics or any particular sport can be monitored by WBAN to improve their performance. WBAN can collect information 
such as the athlete's heart rate and activity. Coaches use such information to improve training as the performance of each muscle can be measured much more accurately $[79,72]$. In the battle field, the moment a soldier is hit, WBAN can transmit the exact location of the wound and his vital signs. WBANs can also monitor carbon monoxide levels for fire fighters. WBANs can even be utilised in advanced animal care. A more comprehensive list of potential applications of WBANs can be found in [109, 151, 72].

\section{Data Communication Characteristics}

WBAN is made up of sensors and actuators located in, on, or very close to the human body. The position of sensors on the body may have an impact on channel quality, i.e. the quality of physical transmission medium. Research shows that the quality of the channel near the human body is very poor and path loss is very high [130]. While the path loss exponent in regular Line-Of-Sight (LOS) situations is about 2, the path loss exponent of the channel near the human body ranges from 2.18 to 3.3 and higher. When two sensors do not have a direct LOS, i.e. Non-Line-Of-Sight (NLOS), the wireless signal absorption of the tissue should be added. Consequently, even more path loss will occur, with path loss exponents of up to 5.8 [130, 132].

The body posture or shape can also influence the channel quality significantly. Due to NLOS and significant change in channel qualities, small movement or a slightly different posture may result in a communication outage. This means that different people in different conditions do not have the same channel quality. Therefore, one of the major challenges for WBANs is the poor channel quality, as it will limit possible solutions. It might be impossible for a patient to have hundreds of sensors attached to her/his body. This is interesting, as it is different from other networks, such as WSNs.

It is worth mentioning that the scope of this thesis is to improve reliability and performance of current commonly used standard in WBANs 
(i.e. IEEE 802.15.4) and it does not intend to either optimise the channel or calculate the position of sensor nodes. Throughout this thesis, we focus on random sensor node locations and the implications of the given channel.

\section{Network Topologies}

WBANs are often considered as convergecast networks. It means that all data packets generated by sensor nodes end up at a single coordinator. Meanwhile control packets (for configuring sensors or handling communication protocol details) are sent by the coordinator towards sensor nodes. The amount of control packets is often significantly smaller than the amount of data packets.

As shown in Figure 2.1, there are two common topologies, namely mesh and star topologies. In mesh topologies, each sensor node is able to relay data packets for the network. In other words, all sensor nodes cooperate to transmit data packets to the coordinator or vice versa. Mesh topology has potential to cope with link outages between sensor nodes and the coordinator. However, it increases traffic load and complexity of the network. Therefore, in WBANs, star topologies are mostly preferred because of their simplicity to manage the network [61, 151]. In this network topology, sensor nodes send data packets directly to the coordinator. In this thesis, star topology is used to set up WBANs.

\subsubsection{Medium Access Control (MAC)}

There are many algorithms for allocating channel access, such as aloha, slotted aloha, Carrier-Sense Multiple Access with Collision Avoidance (CSMA/CA), CSMA with Collision Detection (CSMA/CD) and Time Division Multiple Access (TDMA). Basically they can be divided into two main categories: (1) contention-free MACs (2) and contention-based MACs. In contention-free $M A C$, the protocol ensures that every node in the network access the channel at a given time and interference with other node will never happen. On 


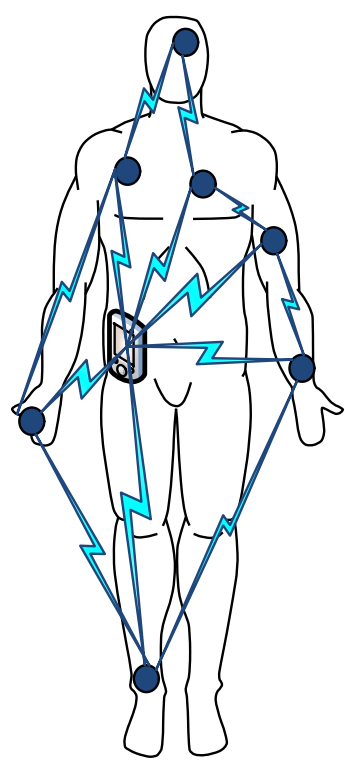

(a) Mesh Topology

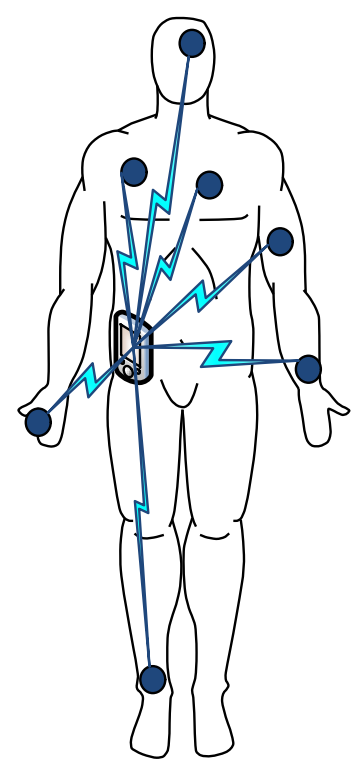

(b) Star Topology

Figure 2.1: Examples of WBAN topologies

the other hand, contention-based MAC protocols allow nodes to compete for accessing the channel and transmitting their data packets.

Contention-free MAC protocols avoid collisions by allocating resources exclusively to nodes. One of the most common approaches to provide contention-free MAC protocols is Time Division Multiple Access (TDMA). TDMA protocols allow multiple devices to use the same frequency band, but they use periodic time windows (called frames), consisting of a fixed number of transmission slots, to separate the medium accesses of different devices. A time schedule indicates which node may transmit data during a certain slot, that is, each slot is assigned to at most one node. The disadvantage of TDMA is that, when the network topology is changed, the slot allocations must also be changed. Further, TDMA protocols can be inefficient in their bandwidth utilisation when slots are of fixed size whereas packet sizes vary and when allocated slots to a node are not used in each iteration. 
Compared with contention-free techniques, contention-based protocols allow nodes to access the medium simultaneously with the possibility of contention. However, they utilise techniques to reduce the possibility of collisions and to recover from them. Commonly used contention-based MAC scheme is the Carrier Sense Multiple Access (CSMA) approach which can be divided as Collision Detection (CSMA/CD) and Collision Avoidance (CSMA/CA).

In CSMA/CD-based schemes, the sender firstly senses the medium to determine whether it is free or busy. If the medium is busy, the sender does not transmit packets. If the medium is idle, the sender can initiate data transmission. In wired networks, the sender is able to keep listening to the medium to detect collisions. It can stop sending data packets whenever it detects collisions. However, in wireless networks, collisions may happen at the receiver, and the sender will not know a collision has occurred. Another problem in wireless networks is hidden-terminal. As shown in Figure 2.2, there are two sensor nodes $A$ and $B$ to reach the coordinator. However, they cannot overhear each other's signals. In case $A$ and $B$ decide to send data packets to the coordinator simultaneously, collision will happen at the coordinator, without $A$ and $B$ directly knowing it. Therefore, CSMA/CA algorithm is often used in wireless networks.

In CSMA/CA, the Collision Avoidance (CA) mechanism is achieved by introducing a random backoff delay before assessing the channel through carrier sensing. Particularly, when the channel is sensed as busy, the sensor node must stop transmitting data packets for a random period of time before listening again for a free communications channel. CSMA/CA also uses Request to Send/Clear to Send (RTS/CTS) to avoid collisions caused by hidden-terminals. In particular, as shown in Figure 2.2, when sensor node $A$ wants to send a data packet to the coordinator, $A$ sends RTS to the coordinator. Then the coordinator broadcasts a CTS. In this way, if a sensor node can hear RTS, it must remain silent for the CTS to be transmitted back to $A$ without conflict. Furthermore, any sensor node, which can hear CTS, 


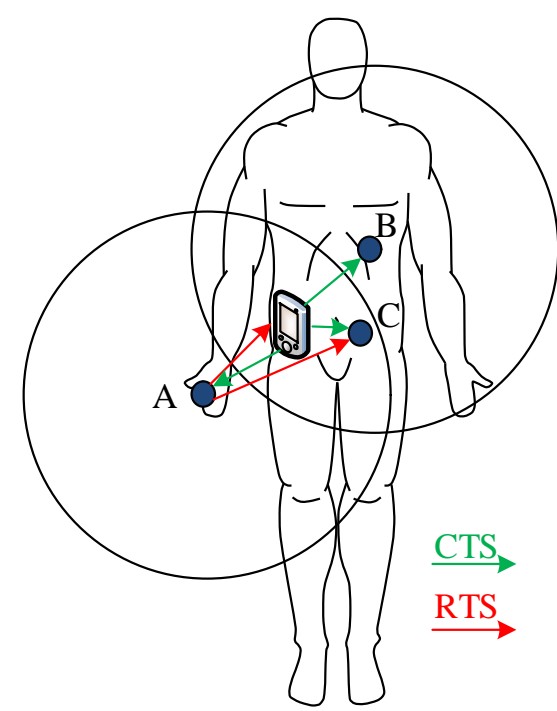

Figure 2.2: $A$ sending an RTS to the coordinator and the coordinator responding with a CTS.

also must remain silent during the upcoming data transmission.

The IEEE 802 Standard comprises a family of networking standards that cover the physical layer and MAC sub-layer specifications of technologies from Ethernet to wireless. For wireless communication the specifications include 802.11 for Wi-Fi, 802.15 for Wireless Personal Area Networks (WPANs), 802.16 for Wireless Metropolitan Area Networks (WMANs) and 802.20 for Mobile Broadband Wireless Access (MBWA). A popular standard for building WBANs is the IEEE 802.15.4 [30]. In the following subsection, we will overview IEEE 802.15.4.

\subsubsection{IEEE 802.15.4}

The IEEE 802.15.4 [3] specification was formed to provide low data rate communications. Low cost, extremely low duty cycle (even below $0.1 \%$ ) and long battery lifetime can be taken into account for this standard as features, too. However, it is yet flexible enough to enable the delivery of periodic data, intermittent data (such as occasional measurements), and 
repetitive low-latency data (for instance, real-time biohazard information). A total of 27 individual channels are dedicated to IEEE 802.15.4 specification. They operate in three different frequency bands: $2.4 \mathrm{GHz}$ ISM band, 868 $\mathrm{MHz}$, and $915 \mathrm{MHz}$. The number of channels dedicated to each frequency band is as follows: one channel for $868 \mathrm{MHz}, 10$ for $915 \mathrm{MHz}$ and 16 for 2.4 $\mathrm{GHz}$. The maximum speed of communication for each frequency band is approximately $20 \mathrm{Kbps}$ per channel (868 MHz band), $40 \mathrm{Kbps}$ per channel (915 MHz band) and $250 \mathrm{Kbps}$ per channel (2.4 GHZ ISM band) [3].

The two types of devices used in IEEE 802.15.4 are specified as Full Function Devices (FFD) and Reduced Function Devices (RFD) [3]. FFD devices are capable of making networks, and have the ability of acting as the coordinator. Communication between these devices is peer to peer (mesh) or through a star configuration. When the network has a star topology, the data packets are directly sent to the coordinator. Different from FFD, RFD devices are only capable of communicating with FFDs [3].

IEEE 802.15.4 supports either of two topologies: the star topology or the mesh topology as shown in Figure 2.1. Star topology will be used in this thesis. In the star topology, a FFD device establishes a wireless network and becomes the coordinator. The coordinator chooses a unique network identifier and then it allows other sensor nodes to join the network.

\section{Beacon-enabled IEEE 802.15.4}

The MAC sub-layer in IEEE 802.15.4 provides beacon management, channel access, Guaranteed Time Slot (GTS) management, frame validation, acknowledged frame delivery, association, and disassociation. IEEE 802.15.4 uses four types of MAC frames: beacon frame, data frame, acknowledgement frame, and MAC command frame. The general MAC frame format is depicted in Figure 2.3, together with the Physical layer (PHY) frame format. The maximum payload for a MAC frame in IEEE 802.15.4 is 102 or 118 bytes, which depends on the addressing scheme and the network topology. In principle, medical data directly could be encapsulated in the payload. 


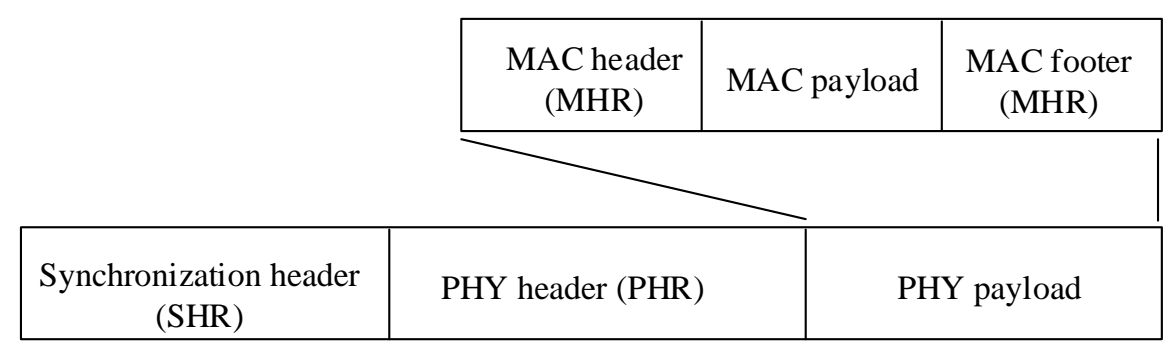

Figure 2.3: IEEE 802.15.4 frame structure

The MAC layer optionally supports a superframe structure, the format of which is defined by the coordinator. Network beacons, which are sent periodically by the coordinator, define the boundary of the superframe, as shown in Figure 2.4. The superframe is divided into two portions, which are active or the Superframe Duration (SD) and an inactive portion. The active portion is divided into sixteen equally sized time slots. Specifically, two values, i.e. macBeaconOrder (BO) and macSuperframeOrder (SO), describes the superframe. $\mathrm{BO}$ describes the interval between two beacons which are sent by the coordinator. This interval is also called the beacon interval (BI). Furthermore, SO describes the length of SD, which is utilised by sensor nodes to communicate with the coordinator. BI and SD are calculated respectively as follows:

$$
B I=\text { aBaseSuperframeDuration } \times 2^{B O} \text { for } 0 \leqslant B O \leqslant 14
$$

$$
S D=\text { aBaseSuperframeDuration } \times 2^{S O} \text { for } 0 \leqslant S O \leqslant B O \leqslant 14
$$

where aBaseSuperframeDuration $(=960)$ is the number of symbols forming a superframe when $\mathrm{SO}$ order is equal to zero. The active portion is composed of three parts: (1) beacon, (2) Contention Access Period (CAP), and (3) Contention Free Period (CFP). The beacon is transmitted, without the use of CSMA, at the start of slot 0 , and the CAP starts immediately following the beacon. 


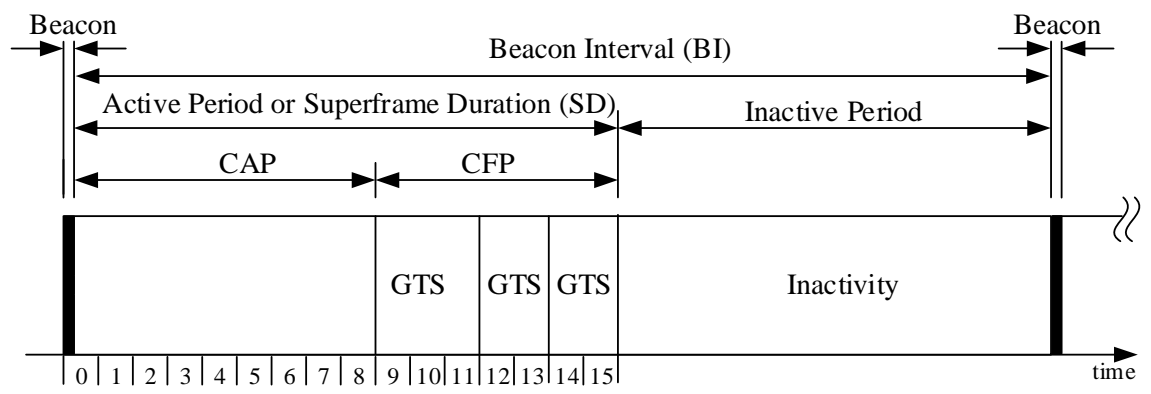

Figure 2.4: Example of the IEEE 80215.4 superframe structure

Each sensor node needs to compete with other sensor nodes using slotted CSMA/CA to access the channel during CAP between two beacons. The coordinator could allocate portions of the active superframe, which are called Guaranteed Time Slots (GTS), to low-latency applications. The GTSs form CFP which always starts at a slot boundary immediately following CAP. Another feature of this standard is unslotted CSMA/CA, which is provided for non-beacon enabled networks, in which there is no superframe structure or GTS. In this thesis, we investigate slotted CSMA/CA and GTS, which are commonly used in WBANs [108, 177].

For a general view, when a sensor node wants to transmit data packets, it first listens for the beacon frame from the coordinator. Sensor node uses the beacon for synchronising with the superframe. At the appropriate time, the sensor node starts transmitting data packets to the coordinator. Finally, when the coordinator receives the data packets properly, it sends back acknowledgement frame to the sensor node.

\section{Carrier Sense Multiple Access with Collision Avoidance (CSMA/CA)}

As mentioned before, CAP starts right after the beacon and finishes before the CFP begins. If the CFP is disabled, the whole active portion will be the CAP. Otherwise, the CAP must be at least aMinCAPLength, which is the minimum number of symbols $(=440)$ forming the CAP. During the CAP, slotted CSMA/CA mechanism is utilised to transfer all frames, except 


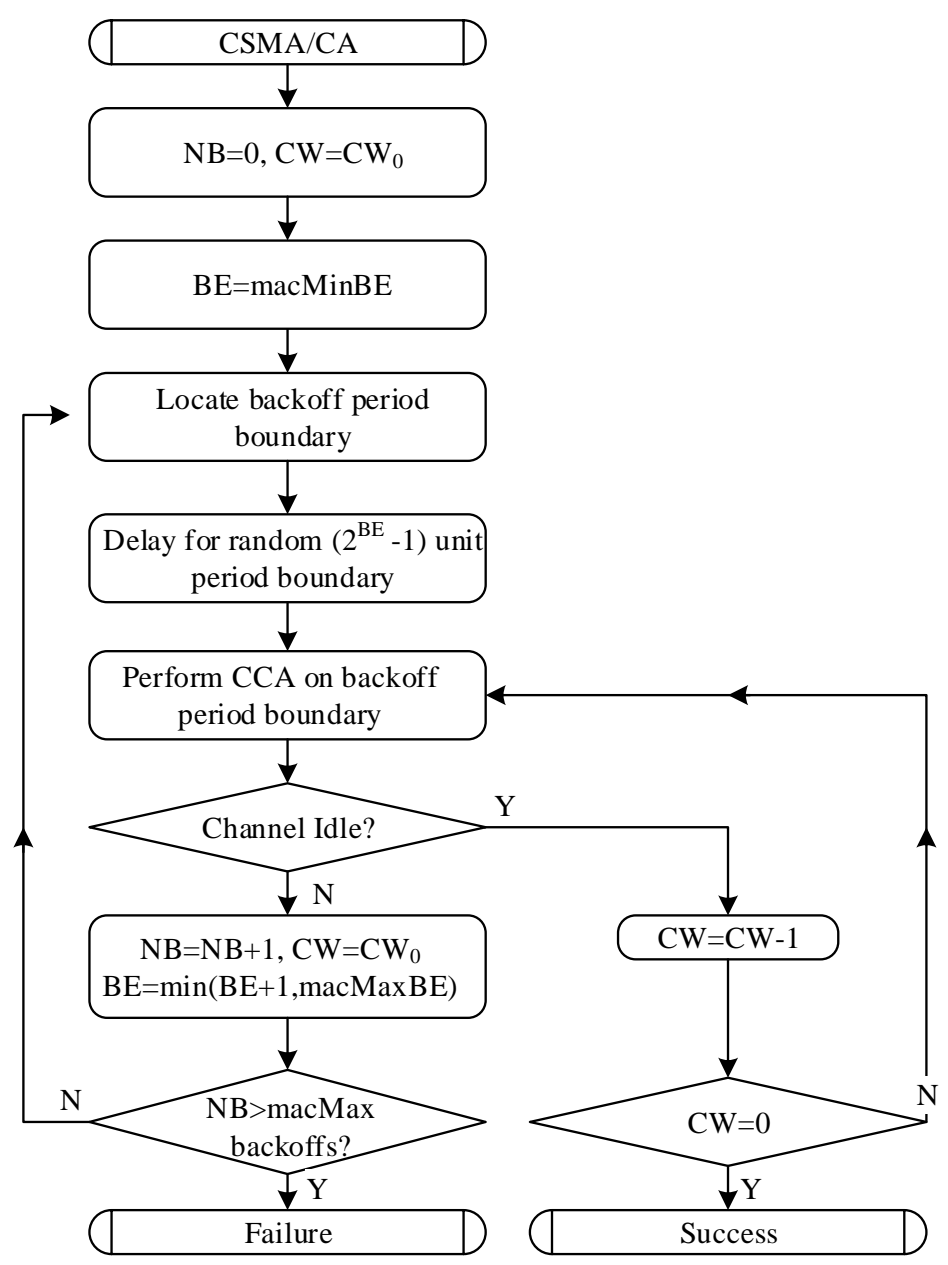

Figure 2.5: Slotted CSMA/CA algorithm in IEEE 802.15.4 [4]

acknowledgement frames, as well as any data frame that quickly follows the acknowledgement of a data request command.

CSMA/CA uses a unit of time called Backoff Period (BP). According to this mode, a node should assess the channel before each transmission (listen before talk). If the channel is idle, the transmission starts. Otherwise transmission will be postponed for a random interval called the backoff delay. Figure 2.5 illustrates slotted CSMA/CA. If CSMA/CA reaches "success", the data packet will be sent; otherwise, it terminates with a channel access 
failure. In the slotted CSMA/CA, there are three key variables for media access control:

- Backoff Exponent (BE): BE holds the number of BPs that a node has to wait before each channel assessment. At the beginning of the CSMA/CA process, BE is initialised to $\operatorname{macMinBE}(=3)$. It is worth to mention that if macMinBE $(=0)$, collision avoidance will be disabled during the first iteration of this algorithm. When a packet is ready for transmission at a node, CSMA/CA forces the node to back off for a random waiting delay to avoid collisions. The random delay is chosen uniformly at random from the range $\left[0,2^{B E}-1\right]$, as shown below:

$$
\text { backofftime }=\operatorname{rnd}\left(0,2^{B E}-1\right) \times B P
$$

After the backoff time is over, if the channel is still busy, BE is incremented by one until macMaxBE (default value of 5 from a valid range of [2...8]) is reached. Afterwards, the value of $\mathrm{BE}$ will be capped at $\operatorname{macMaxBE}$.

- Contention Window (CW): CW defines the number of backoff periods that the channel is required to be clear before starting each transmission. $\mathrm{CW}$ is initialised to $\mathrm{CW}_{0}(=2)$. It will be reset to $\mathrm{CW}_{0}$ whenever the channel is sensed as busy. On the other hand, if the channel is sensed as clear, CW is decremented by one and this is repeated until $\mathrm{CW}$ reaches zero. After that, data transmission shall start.

- Number of Backoffs (NB): NB counts the number of backoff attempts experienced for the current transmission attempt. It is initialised to zero $(\mathrm{NB}=0)$ for any new transmission. Every time the channel is assessed as busy, NB is incremented by one. The algorithm will not allow NB to exceed macMaxBackof $f s(=4)$. If $\mathrm{NB}=$ macMaxBackof $f s$, a transmission failure is declared. Otherwise, the backoff procedure will continue. 
IEEE 802.15.4 ensures that the whole procedure of CSMA/CA and transactions can finish before the end of the CAP. For example, IEEE 802.15.4 stops the CSMA/CA procedure, whenever the number of backoff periods is greater than the remaining number of backoff periods in the CAP. In this case, IEEE 802.15.4 will proceed to check whether the rest of the CSMA/CA procedure, such as two Clear Channel Assessment (CCA) analyses, data packet transmission and any acknowledgement, can be completed before the end of the CAP. If so, it can proceed; otherwise, it needs to wait until the start of the CAP in the next superframe.

\section{Guaranteed Time Slot (GTS) Allocation and Management}

In IEEE 802.15.4, the coordinator is able to allocate a portion of the superframe, called GTS, to a sensor node. If there is sufficient capacity in the superframe, a maximum of seven GTSs is allocated by the coordinator to sensor nodes within the CFP. The coordinator must store all the information necessary to manage seven GTSs such as the starting slot, length, direction, and associated device address.

In IEEE 802.15.4, a sensor node sends a GTS allocation request to the coordinator using CSMA/CA during CAP. As shown in Fig. 2.6, the sensor node indicates the required bandwidth in the request. On receipt of the GTS allocation request, the coordinator determines whether the requested GTS can be allocated based on the current available capacity in the superframe so that the length of CAP must not be shorter than aMinCAPLength. Furthermore, the coordinator is only allowed to allocate a maximum of seven GTSs within a superframe. By default, the coordinator assigns GTSs to sensor nodes in a First-Come-First-Served (FCFS) fashion, provided there is sufficient bandwidth available.

In the case that there is sufficient capacity for the requested GTS, the coordinator creates a GTS descriptor. The GTS descriptor contains the requested specifications, the short address of the requesting sensor node, the start slot and the length. 


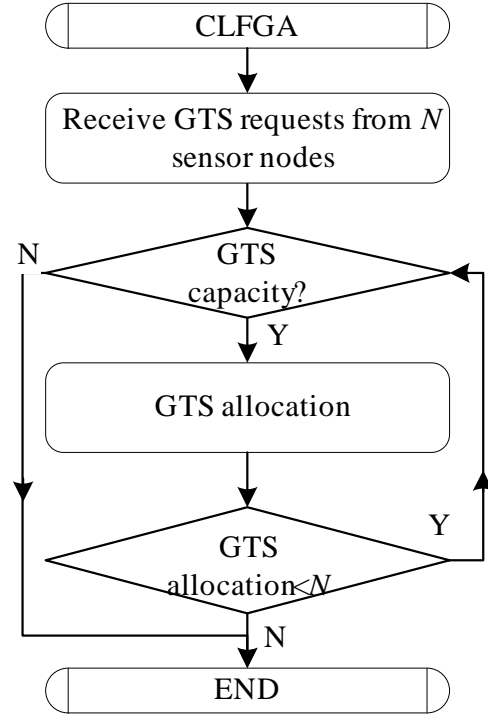

(a) The flow chart of GTS allocation algorithms

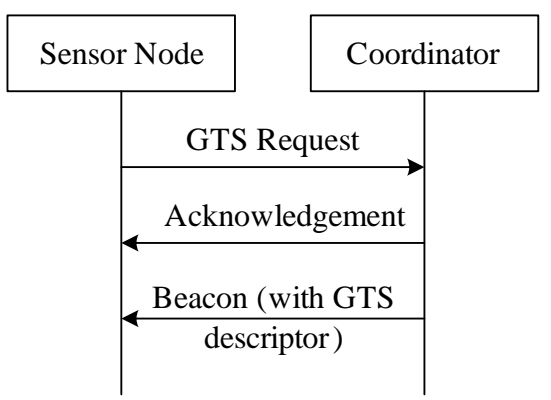

(b) Sequence chart of GTS allocation initiated by a sensor node

Figure 2.6: IEEE 802.15.4 GTS allocation

On receipt of the acknowledgement, the sensor node waits for the upcoming beacon from the coordinator. Once it is received, the sensor node will proceed to transmit data based on the GTS descriptor generated by the coordinator. In the case that sensor node realises the transmission cannot be completed before the end of the current GTS, it postpones the transmission to the specified GTS in the next superframe. If the sensor node misses the beacon at the beginning of a superframe, it cannot use its GTSs and must wait for the next beacon.

GTS deallocation can be performed by either the coordinator or the sensor node. For the sensor node initiated deallocation, sensor nodes will send GTS deallocation request to the coordinator using CSMA/CA during CAP. For the coordinator initiated deallocation, the coordinator should first detect sensor nodes that have stopped using GTS. If the GTSs allocated to a sensor node has not been utilised for $2 \times n$ superframes, the coordinator deallocates the GTSs from the sensor node. Here $n$ is defined as: 


$$
n= \begin{cases}2^{8-B O}, & \text { if } 0 \leqslant B O \leqslant 8 \\ 1, & \text { if } 9 \leqslant B O \leqslant 14\end{cases}
$$

\subsubsection{Fuzzy Logic Controller (FLC)}

Designing controller for complex and dynamic system is a challenging task [1, 112]. To design effective controllers, soft computing techniques such as fuzzy logic has been proven to be highly useful by imitating how human operators control complex systems based on rudimentary system knowledge.

For the first time, Lofti A. Zadeh introduced fuzzy logic and fuzzy set in 1965 [174] and 1973 [175], respectively. Since its invention, fuzzy logic has attracted substantial research attention and become a successful alternative to classical control techniques. The key characteristics of this success can be listed as follows:

- Flexibility with information sources. FLCs can use both sensor data and human experts, or just one if the other is not available. This feature brings flexibility to FLCs with wide range of applications.

- Model-free: FLCs are model-free controllers, which are independent of the physical system model. This characteristic enables FLCs to cope with systems without mathematical models.

- Universal approximation: Fuzzy systems are universal approximators, which make them suitable for non-linear control system design.

- Desirable compromise between performance and cost: When FLCs are implemented in general-purpose processors, they usually need low computational resources. Another advantage of using FLCs is easy understandability, which is important for non-experts. 
Fuzzy rule-based models, which are applications of fuzzy set theory, are commonly used to provide frameworks for engineering applications. There are three types of rule-based models:

- Linguistic fuzzy model which is the original Zadeh's model of fuzzy rule base. Linguistic fuzzy model contains both the antecedent and consequent part of IF-THEN rule [175]. This model is still popularly used because of its flexibility in handling various practical applications.

- Fuzzy relational model proposed by Pedrycz [123]. This model is able to associate a particular antecedent proposition with several different consequent propositions through a fuzzy relation.

- Takagi-Sugeno fuzzy model, proposed by Takagi et al. [146]. The consequent in this model is a crisp function of antecedent variables.

Six classifications of FLCs based on differences in fuzzy rules and methods of their generation can be defined as follows: [46]:

- Fuzzy Proportional-Integral-Derivative (PID) control.

- Hybrid techniques encompassing fuzzy logic, neural networks, evolutionary algorithms, etc.

- Fuzzy-sliding mode control.

- Adaptive fuzzy control.

- TakagiSugeno model-based fuzzy control.

- Conventional (Mamdani) fuzzy control.

\section{Fuzzy Proportional-Integral-Derivative (PID) control}

Due to simplicity and low cost of implementation, Proportional-IntegralDerivative (PID) controllers are commonly used in industrial applications. 
As PID control might not cope with highly nonlinear and uncertain systems, they are combined with FLCs which are able to handle non-linearities and uncertainties through use of fuzzy set theory [46]. In general, fuzzy PID controllers outperform conventional PID controllers [112].

\section{Hybrid Techniques}

Hybrid techniques use two or more different soft-computing techniques simultaneously to improve the control effectiveness. For example, Neural Network (NN) controls and FLCs are both model-free controls. NN controls acquire knowledge mainly through data training (or learning). On the other hand, FLCs acquire qualitative and imprecise knowledge mainly through the expert. Consequently a combination of them, which is called Neuro-Fuzzy Control (NFC), enables both (1) high learning capabilities and computation efficiency and (2) a powerful framework for expert knowledge representation. Consequently, NFCs have been widely used to design the membership functions in the fuzzy control system [70, 117, 104].

\section{Fuzzy sliding mode control}

It has been shown that sliding-mode control can provide robust control on nonlinear application with uncertainties, such as robotic applications, and many Multiple-Input Multiple-Output (MIMO) systems [154, 179]. However, it suffers from the chattering phenomena because of its sharp switching criterion around the sliding surface. This phenomena is undesirable because it can excite the high frequency dynamics of the system. Chattering in sliding mode control can be compensated by defining fuzzy boundary layers that replace sharp (crisp) switching surfaces [60, 46]. Fuzzy sliding mode control can also use EC techniques to design membership functions $[28,95]$. 


\section{Adaptive fuzzy control}

Adaptive control aims at controlling initially uncertain systems. Mostly researchers try to design adaptive controllers based on the assumption of linear or simplified non-linear mathematical models [71, 90]. Since mathematical models might not be available for many complex systems, adaptive control systems might lose their effectiveness. As mentioned before, FLCs are particularly effective on non-linear systems [160]. In adaptive fuzzy controllers, FLCs are utilised to model those unknown nonlinear functions. The parameters of FLC, such as membership functions, can be updated based on some adaptive laws which are derived from the Lyapunov stability theory [46].

\section{TakagiSugeno model-based fuzzy control}

Engineering applications often have numerical values for both input and output. Accordingly, FLCs utilise fuzzifiers to transfer crisps into linguistic variables and defuzzifiers to translate a quantifiable linguistic variables back into crisps. In contrast, Takagi and Sugeno [146] proposed FLCs that only use fuzzifiers to process input variables. The output variables in Takagi-Sugeno fuzzy controllers are a linear combination of input variables. In other words, the antecedent in IF-THEN rules is fuzzy but the consequent part is a linear combination of input variables, as follows:

$$
\begin{aligned}
& \text { IF } x_{1} \text { is } a_{1}^{i} \text { and } x_{2} \text { is } a_{2}^{i} \text { and } \ldots \text { and } x_{n} \text { is } a_{n}^{i} \\
& \text { THEN y is } a_{0}+a_{1} x_{1}+a_{2} x_{2}+\ldots+a_{n} x_{n}
\end{aligned}
$$

where $x$ and $y$ are input and output variables, respectively. The major advantage of using Takagi-Sugeno fuzzy controllers is reduced number of fuzzy rules $[78,145,46]$.

\section{Conventional (Mamdani type) fuzzy logic control}

Conventional (Mamdani type) fuzzy logic control has been widely used in many practical applications such as robots $[14,149,166,170]$, geomag- 


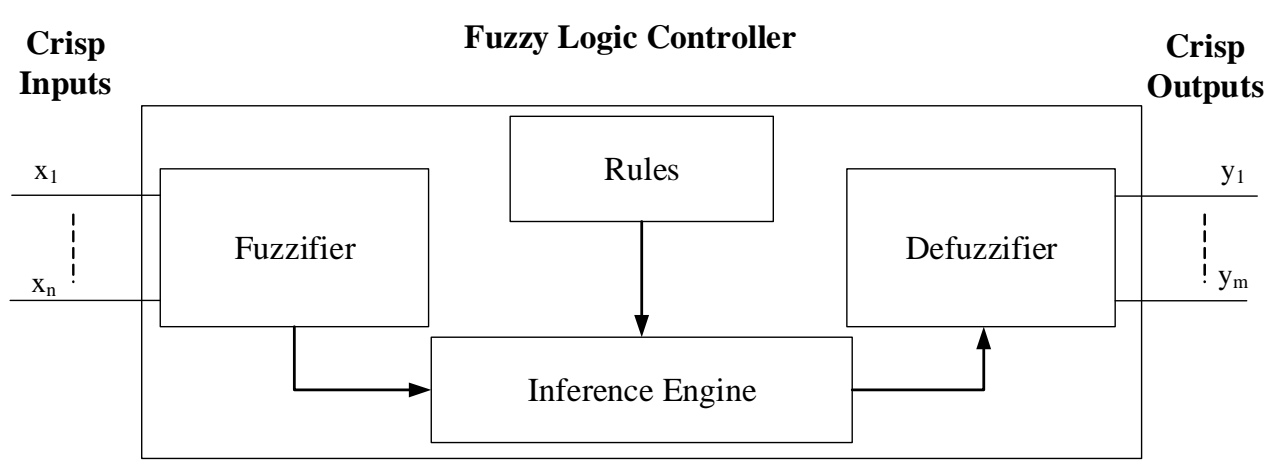

Figure 2.7: Basic diagram of the Fuzzy Logic Control System

netically induced currents [68], stirred tank reactors [85], Hybrid Electric Vehicles [172, 15], smart power management [148] and localisation in WSNs [117]. Although Takagi-Sugeno fuzzy controls can be simple in structure and number of fuzzy rules, the lack of fuzziness in the consequent might make it difficult to interpret for human experts. On the other hand, conventional FLCs overcome this challenge by introducing linguistic variables for both input and output variables. Another advantage of conventional FLCs is regarding their flexibility in using different fuzzy operators to perform fuzzy inference [34]. These advantages explain the popularity of this type of FLCs in many practical applications [34].

As show in Figure 2.7, the basic components of a conventional FLC can be listed as follows [34]:

- Fuzzification: This component uses a fuzzifier to translate crisp input values into fuzzy linguistic values used in the fuzzy inference engine.

- Knowledge base: This component contains a collection of rules acquired by expert knowledge.

- Fuzzy inference engine: This component is the heart of FLCs. The fuzzy inference engine uses fuzzy set theory involving control fuzzy logic operations to combine the fuzzy rules in the rule base and map inputs to outputs. 
- Defuzzification: This component translates fuzzy output linguistic values into the crisp values. The common defuzzifiers are Centre Of Gravity (COG), Centre Of Average (COF) and maximum.

In contrast with Takagi-Sugeno FLCs, in Mamdani FLCs both antecedent and consequent of IF-THEN rules are fuzzy as follows:

$$
\begin{aligned}
& \text { IF } x_{1} \text { is } a_{1}^{i} \text { and } x_{2} \text { is } a_{2}^{i} \text { and } \ldots \text { and } x_{n} \text { is } a_{n}^{i} \\
& \text { THEN } y_{1} \text { is } b_{1}^{i} \text { else } y_{2} \text { is } b_{2}^{i} \text { else ... else } y_{m} \text { is } b_{m}^{i}
\end{aligned}
$$

where $a_{j}^{i}(j=1,2, \ldots, n)$ and $b_{j}^{i}(j=1,2, \ldots, m)$ denotes the fuzzy sets for input $\left(x_{k}, k=1,2, \ldots, n\right)$ and output $\left(y_{k}, k=1,2, \ldots, m\right)$ variables respectively.

This thesis utilises Mamdani FLCs for media access control in WBANs. FLCs have potential to improve communication quality, however, they still face two main problems: (1) designing an adequate knowledge base for the controller, (2) selecting and adjusting key control parameters. The knowledge base is often derived from expert knowledge [1]. There are two main methods to design FLCs:

- Manual design by human experts. In this approach, FLC specifications such as the linguistic labels associated with linguistic variables, structure of the rule base, and meaning of each label, are designed by domain experts.

- Automatic design by learning methods. In this approach, automated learning methods such as Evolutionary Computation (EC) techniques, ad-hoc data driven generation methods, variants of the least squares method, gradient descent method, neural networks, and clustering techniques, are used to design FLCs. Among these methods, the most commonly used approach to automatically designing FLCs is based on EC techniques [159, 47]. 


\subsubsection{Evolutionary Computation (EC)}

EC is a soft computing technique which is inspired by biological evolution. There are three main classes of EC algorithms [42]: Evolutionary Algorithms (EAs) [42], Swarm Intelligence (SI) [41, 82] and Cooperative Coevolution (CC) [42].

\section{Evolutionary Algorithms (EAs)}

EAs are heuristic and population based. Each individual of the population is considered as a potential candidate solution. To measure the quality of each individual, a fitness value will be assigned. Then new population of candidate solutions is produced by stochastically removing less desired solutions and introducing random new candidate solutions by the use of techniques inspired by biological evolution: reproduction, mutation, crossover, and selection [42]. The well-known EAs, namely genetic algorithms, genetic programming, evolutionary strategies, evolutionary programming differential evolution, are briefly explained as follows:

\section{- Genetic Algorithms (GAs)}

GA is the most widely known EAs and was initially introduced by John Holland [62, 63, 12]. In GA, each candidate solution consists of a fixed-length array of bits, called chromosomes. GAs evolve the population of candidate solution by the mean of genetic operators to search for the optimal solution for a given problem. These genetic operators include crossover, mutation, and reproduction.

\section{- Genetic programming (GP)}

GP [87] is different from standard EAs in representation. As mentioned before, GA is represented as a fixed-length array of chromosomes. However, GP is represented using trees as chromosomes. Specifically, GP contains a population of computer programs, which 
is optimised according to a fitness landscape [42,88]. Due to its flexibility, GP has been successfully used to solve many optimisation problems such as classification, regression and scheduling [50,44].

\section{- Evolutionary Strategies (ESs)}

ESs were introduced by Rechenberg and Schwefel for shape optimisation problems [42]. They utilise natural problem-dependent representations with a fixed-length real-valued vector to represent each candidate solution. Particularly, both the genetic building blocks and strategy parameters (i.e. to simulate the behaviour of that individual) jointly form such a vector. ESs evolve the population based on both the genetic characteristics and the strategy parameters [16].

\section{- Evolutionary Programming}

EP was introduced by Fogel et al. [12, 48] to simulate evolution as a learning process with the aim of evolving Finite-State Machines (FSMs). The main difference between GP and EP is the structure of the program, which is fixed in EP. The main operations of EP are mutation and selection operators. In contrast with GAs, EP does not originally include crossover operators.

\section{- Differential evolution}

DE was introduced by Storn and Price [144] as a powerful EA to optimise nonlinear and non-differentiable continuous space functions. DE, similar to GAs, evolves generations of candidate solutions. The major difference between GAs and DE lies in the mutation operators. $\mathrm{DE}$ uses the difference between a pair of chosen vector and a scaling factor to move through the search space $[150,128]$.

\section{Swarm Intelligence}

Swarm Intelligence (SI) algorithms are inspired by the collective intelligence of social behaviour of simple agents such as bird flocks, ant colonies, and 
bacterial growth $[7,82,103]$. Generally, SI uses each agent for parallel local search in hope of finding optimum solutions. In the meantime, each agent interacts with other agents to reach global behaviour. Two well-known SI techniques are ant colony optimisation and particle swarm optimisation.

\section{- Ant Colony Optimisation}

Ant colony optimization (ACO) [40,39] was introduced by Dorigo [42] to mimic the behaviour of ants looking for the shortest path from their colony to food [38]. In ACO, every ant can produce a candidate solution. These ants leave pheromones as footprint on the ground. Therefore, other ants in the colony can follow the path and reach the food. In ACO, the best solution is the "path" that has the most pheromones. In this way, ACO can take advantage of the elements of previous solutions.

\section{- Particle Swarm Optimisation}

Although Particle Swarm Optimisation (PSO) and the technical terminology are grounded in physical particles, it is inspired by social behaviour of bird flocking or fish schooling [41, 81, 158, 125]. Specifically, each candidate solution is represented as a particle. In PSO, particles move in the search space to find the optimum solution. In contrast to EAs, PSO does not support crossover and its mutation is defined through a vector addition. Particularly, PSO updates the position of each particle based on the best experience of its own and its neighbouring particles to move the whole swarm towards the optimal solution. PSO has been applied to solving problems in a variety of areas $[42,57,10,6]$, because of its simplicity and effectiveness $[158,125]$.

\section{Cooperative Coevolution}

Cooperative Coevolutionary (CC) techniques use multiple sub-populations, each representing part of a problem, to cooperatively solve a large problem. 
CC has been successfully applied to solving high-dimensional function optimisation [126] and job shop scheduling problems [66]. Specifically, CC adopts a divide and conquer strategy to decompose any given problem into sub-populations, each of which can be solved effectively. When individuals from one sub-population work well with individuals from other sub-populations, they will be rewarded. Penalties will apply otherwise. One of the well-know CC techniques is Cooperative Particle Swarm Optimisation (CPSO) [156, 92].

In this thesis, we have utilised DE, PSO and CPSO, which are explained in subsections 2.1.6, 2.1.7 and 2.1.8, respectively, to automatically design FLCs in WBANs.

\subsubsection{Differential Evolution (DE)}

The DE algorithm [144] is similar to other traditional evolutionary algorithms, such as GAs. It supports the commonly used genetic operators including crossover, mutation and selection. The major difference between $\mathrm{DE}$ and GA is the mutation operator.

Like nearly all EAs, DE randomly initiates a population of NP Ddimensional candidate solutions. The $i$ th candidate solution of the population at time $t$ is denoted as follows:

$$
\vec{X}_{i}(t)=\left[x_{i, 1}(t), x_{i, 2}(t), \ldots, x_{i, D}(t)\right], i \in 1,2, \ldots N P
$$

A mutated candidate solution in DE is created according to the following:

$$
\left.\overrightarrow{M_{i}}(t+1)=\overrightarrow{X_{r 1}}(t)+F \times \overrightarrow{X_{r 2}}(t)-\overrightarrow{X_{r 3}}(t)\right)
$$

where $r 1, r 2$ and $r 3$ are different (non-identical) indexes randomly selected from $\{1, \ldots, \mathrm{NP}\}$, and $F$ is a scaling factor where $F \in[0,2] . \vec{M}_{i}(t+1)$ and $\overrightarrow{X_{r 1}}$ are called the target and base candidate solutions, respectively. "Binomial" 
crossover is used to increase the potential diversity of the population. In the crossover operation, the trial vector is first determined as below:

$$
\begin{gathered}
\vec{U}_{i}(t)=\left[u_{i, 1}(t), u_{i, 2}(t), \ldots, u_{i, D}(t)\right] \\
l=\operatorname{rand}(1, D) \\
u_{i, j}(t+1)= \begin{cases}m_{i, j}(t+1), & \text { if } \operatorname{rnd}_{j}(0,1) \leq C r \text { or } j=l \\
x_{i, j}(t), & \text { if } \operatorname{rnd}_{j}(0,1)>C \text { or } j \neq l\end{cases}
\end{gathered}
$$

for each $j \in\{1,2, \ldots, D\}$. $\mathrm{Cr}$ is the crossover constant, which is [0...1]. Selection as the final step decides whether the target vector or the trial vector should become a member of the next generation, as below:

$$
\vec{X}_{i}(t+1)= \begin{cases}\vec{X}_{i}(t), & \text { if } f\left(\vec{U}_{i}(t+1)\right) \leq f\left(\vec{X}_{i}(t)\right) \\ \vec{U}_{i}(t+1), & \text { if } f\left(\vec{U}_{i}(t+1)\right)>f\left(\vec{X}_{i}(t)\right)\end{cases}
$$

where $f()$ is a fitness function to be minimised.

\subsubsection{Particle Swarm Optimization (PSO)}

PSO is an evolutionary algorithm based on the social behaviour of birds within a flock [81]. It is composed of candidate solutions called particles where the whole population of particles is a "swarm". Each particle occupies a position vector $\vec{X}_{i}$ and a velocity vector $\vec{V}_{i}$ in $D$-dimensional hyperspace. The position vector represents a candidate solution in the search space, which is initialised randomly at the beginning. The particle possesses its current position $\vec{X}_{i}$ and velocity $\vec{V}_{i}$. In addition, it memorises its previous best position called personal best, pbest, and the position of the best particle in the whole swarm called gbest. In each iteration $t$, PSO updates the velocity of the $i$ th particle in the $d$ th dimension as follows: 
$v_{i d}(t+1)=\omega \times v_{i d}(t)+c_{1} \times r_{1}(t)\left(\right.$ pbest $\left._{i d}(t)-x_{i d}(t)\right)+c_{2} \times r_{2}(t)\left(g b e s t_{d}(t)-x_{i d}(t)\right)$

where $c_{1}$ and $c_{2}$ are "self-confidence" and "swarm confidence" constants respectively. $r_{1}(t)$ and $r_{2}(t)$ are random numbers uniformly distributed in [0...1]. Accordingly, the position of the $i$ th particle in the $d$ th dimension is updated as follows:

$$
x_{i d}(t+1)=x_{i d}(t)+v_{i d}(t+1)
$$

The personal best position of each particle is updated as follows:

$$
\text { pbest }_{i d}(t+1)= \begin{cases}\text { pbest }_{i d}(t), & \text { if } f\left(x_{i d}(t+1)\right) \leq f\left(\text { pbest }_{i d}(t)\right) \\ x_{i d}(t+1), & \text { if } f\left(x_{i d}(t+1)\right)>f\left(\text { pbest }_{i d}(t)\right)\end{cases}
$$

where $f()$ is a fitness function to be minimised. The update process is iteratively repeated until either an acceptable gbest is achieved or a fixed number of iterations $t_{\max }$ is reached.

\subsubsection{Cooperative Particle Swarm Optimisation (CPSO)}

CPSO [156], which is explained in Algorithm 1, decomposes a position vector into $K$ s-dimension subcomponents (where $D=K \times s$ ). In order to evaluate the fitness value of a particle, CPSO concatenates all gbest particles from $K$ different sub-populations. Function $b\left(j, P_{j} . x_{i}\right)$ is called to evaluate the $i$ th particle in the $j$ th sub-population. This function returns an $D$-dimensional vector as the whole solution containing gbest with its $j$ th component replaced by $P_{j} . x_{i}$. The idea is to evaluate how well $P_{j} . x_{i}$ "cooperate" with another sub-population. 
define $: b(j, z) \equiv\left(P_{1} . g b e s t, \ldots, P_{j-1}\right.$. gbest $\left., z, P_{j+1} . g b e s t, \ldots, P_{K} . g b e s t\right)$

Create and initialise $K$ swarms, each with $s$ dimensions (where $D=K \times s)$;

The $j$ th swarm is denoted as $P_{j}, j \in[1 . . K]$;

repeat

for each swarm $P_{j} \in[1 . . K]$ do

for each candidate $F L C i \in[1 . . s]$ do

if $f\left(b\left(j, P_{j} . x_{i}\right)\right)<f\left(b\left(j, P_{j}\right.\right.$. pbest $\left.\left._{i}\right)\right)$ then

$P_{j}$.pbest $t_{i} \leftarrow P_{j} . x_{i} ;$

end

if $f\left(b\left(j, P_{j}\right.\right.$. pbest $\left.\left.j\right)\right)<f\left(b\left(j, P_{j}\right.\right.$. gbest $\left.)\right)$ then $P_{j}$. gbest $\leftarrow P_{j}$.pbest ${ }_{j} ;$

end

end

Perform PSO updates, i.e. velocity and position, on $P_{j}$;

end

until termination condition is true;

Algorithm 1: The pseudocode of the CPSO algorithm. $P_{j} . x_{i}$ denotes the current position of the $i$ th candidate solution of the $j$ th sub-population. $P_{j}$.pbest $t_{i}$ is the personal best of the $i$ th candidate solution of the $j$ th subpopulation. The $j$ th of the $K$ sub-populations has a global best particle $P_{j}$. gbest. 


\subsection{Literature review}

This section reviews the literature related to different methods proposed in this thesis. Subsection 2.2.1 first presents related work about MAC protocols in WBANs. Subsection 2.2.2 reviews cross-layer FLCs in WBANs. Subsections 2.2.3 and 2.2.3 discuss FLC design and its application to WBANs.

\subsubsection{Medium Access Control (MAC)}

MAC protocols that are specifically developed for WBANs are fairly limited. Since WBANs and WSNs are similar in many aspects, it is useful to study WSNs MAC as well. Researchers often try to optimise the energy consumptions in WSN MAC protocols. For example, Ye et al. introduced S-MAC [171] which enables sensor nodes to transmit data packets within divided time slots through virtual clusters. The authors tried to balance between energy and packet delay. Dam et al. [155] improved S-MAC by introducing T-MAC which adaptively changes the duty cycle based on different traffic patterns. Although this adaptability removes a conservative slot schedule for the worst traffic pattern in S-MAC, the aggressive sleep schedule reduces the throughput and increases packet delay. Krishnamachari et al. [98] also used the adaptive duty cycle approaches while designing D-MAC. However, all these MAC protocols, i.e. S-MAC, T-MAC, and D-MAC, introduce traffic load for synchronising and scheduling sleep periods. Moreover, WSNs MAC protocols are typically designed for multi hop ad hoc networks, while this thesis focuses on star-topology WBANs.

IEEE 802.15.4 [3] is the most commonly used standard to implement WBANs $[26,91,83]$. Since most WBANs are built based on IEEE 802.15.4 compliant chip set [91,83], the IEEE 802.15.4 MAC protocol is often used in WBANs. However, previous research showed that IEEE 802.15.4 can be very limited in terms of its reliability (measured by Packet Delivery Ratio (PDR)), and performance (measured by throughput and packet delay) $[152,120,53,24,113,25,54]$. Therefore, efforts have been made to improve 
reliability and performance of IEEE 802.15.4. They can be divided largely into two categories: (1) improving the MAC protocol during only CAP and (2) improving the MAC protocol while CFP is enabled. The former focuses on enhancing the effectiveness of CSMA/CA in IEEE 802.15.4 and the latter tries to improve GTS allocation in IEEE 802.15.4.

\section{IEEE 802.15.4 CSMA/CA}

IEEE 802.15.4 recommends a popular technology known as Carrier Sense Multiple Access with Collision Avoidance (CSMA/CA) to schedule concurrent channel access [3]. In CSMA/CA, the Collision Avoidance (CA) mechanism is achieved by introducing a random backoff delay before assessing the channel through carrier sensing. Particularly, when the channel is assessed as busy, the maximum backoff delay will be increased exponentially. For each new transmission, CSMA/CA in IEEE 802.15.4 ignores the most recent channel condition. It blindly restarts the whole backoff process instead of adjusting backoff delay based on the recent channel condition. In other words, exponential backoff algorithm in IEEE 802.15.4 is memory-less [8] and cannot consider the channel condition history. As a result, while the channel is still busy, the collision rate might increase and accordingly throughput would decline.

To address this issue, several amendments have been proposed to update the maximum backoff delay in a more adaptive manner [58]. For example, the Enhanced Collision Resolution (ECR) and the Enhanced Backoff (EB) mechanisms are proposed in [58] to improve throughput of CSMA/CA. The authors suggested that two consecutive CCAs are not a good indication of channel condition. Consequently, ECR increases BE after a number of consecutive CCAs which is set to macMaxBackoffs. Moreover, instead of resetting the maximum backoff delay after a transmission failure, the maximum backoff delay remains high and will only be reduced after a successful transmission. In this way, ECR tries to preserve the channel condition history by slowly decreasing BE. On the other hand, EB shifts 


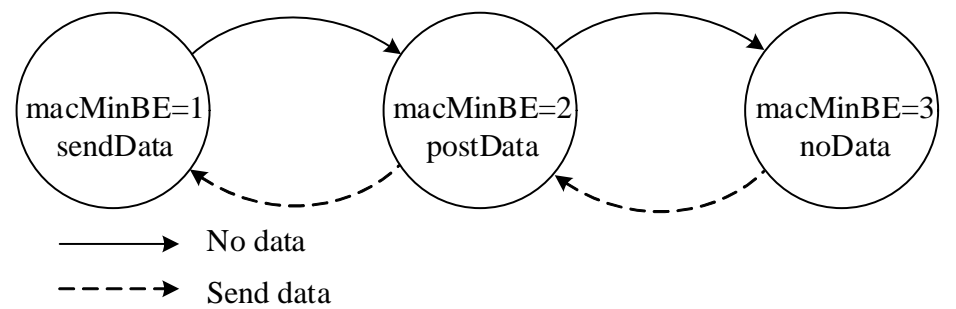

Figure 2.8: State transition scheme to adjust macMinBE in IEEE 802.15.4, proposed by Ko et al. [51]

the range of backoff counters based on the expected number of busy BPs to avoid overlaps among different backoffs and CCAs. Although this approach makes CSMA/CA more effective in busy channels, the performance of the network might be poor, especially if the channel condition fluctuates frequently. Furthermore, average delay may also increase subject to the shifting range of BP [163].

Ko et al. [51] proposed a state transmission scheme to dynamically adjust macMinBE based on the transmission conditions. In this scheme, the macMinBE value changes in the range of [1..3], as the condition of the sensor node changes. As shown in Figure 2.8, each sensor node has three different states with different macMinBE values. This scheme calculates the channel access frequency by keeping the records of the number of idle beacon frames (with no transmission) and the number of successful transmissions within a beacon frame in each sensor node. This frequency is then used to prioritise each sensor node to access the channel. Although this scheme tried to consider the frequency of channel access with respect to each sensor node, it cannot quickly adapt itself to the current channel condition.

In order to compensate for the injected delay, Wong et al. [163] proposed an Additional Carrier Sensing (ACS) method. They used extra channel assessments before increasing backoff delay to collect more information regarding the channel condition. Specifically, the authors suggested that 
there are two reasons for the second CCA failure in IEEE 802.15.4. (1) when sensor node $A$ performs the first CCA, sensor node $B$ in the network also successfully performs its second CCA in the same time. In this case, when sensor node $A$ tries to perform CCA for the second time, sensor node $B$ is in middle of sending its data packets and the channel is busy. Therefore, the second CCA for sensor $A$ fails. (2) Sensor node $A$ performs CCA for the first time, while sensor node $B$ is waiting for the acknowledgement after finishing its transmission. Therefore, the first CCA shows a clear channel and then sensor node $A$ performs its second CCA. In the meantime, if the transmission for sensor $B$ is successful, the coordinator sends back acknowledgement and the channel is busy. In view of the two reasons, ACS requires sensor nodes to perform the third CCA to reduce the likelihood of CCA failure. In this way, sensor node $A$ has another opportunity to access the channel after sensor node $B$ receives it acknowledgement. However, these studies tend to ignore application layer information. Thus, backoff delay might not be adapted based on the specific application requirements of each sensor node.

\section{IEEE 802.15.4 GTS}

As mentioned in Subsection 2.1.3, IEEE 802.15.4 also supports TDMA based on the GTS mechanism. GTS can guarantee the reliability and performance of data delivery by allocating a specific time slot within a superframe for data transmissions. However, existing scheme in IEEE 802.15.4 faces some limitations as follows:

- In IEEE 802.15.4 only up to seven GTSs can be allocated during each superframe.

- GTS allocation is based on simple First-Come, First-Served (FCFS) mechanism.

Specifically, in IEEE 802.15.4, sensor nodes send GTS requests to the coordinator and the coordinator allocates GTS to a limited number of sensor 
nodes in a simple FCFS fashion. This strategy might lead to underutilisation of the GTS bandwidth resources, therefore, limiting the reliability and performance of WBANs. Several research works tried to address the limitation related to CFP [86, 177, 135, 64]. For example, Koubaa et al. [86] proposed an Implicit GTS Allocation MEchanism (i-GAME) to improve the GTS utilization efficiency by accepting multiple flows sharing the same GTS. Particularly, in this algorithm, each sensor node requests a guaranteed service by sending its traffic specification and delay requirement. Then the coordinator tries to allocate bandwidth through GTS based on the traffic specification of the requesting nodes, their delay requirements, and the available GTS resources. The $i$-GAME shares multiple traffic flows within the same GTS to minimise the amount of wasted bandwidth, while still trying to meet their delay requirements. However, in the $i$-GAME, since the algorithm starts the GTS allocation from the last time slot in a round-robin manner, it may fail to serve a flow with hard real-time deadline, which needs to be assigned to the first GTS in the CFP [165]. Additionally, it requires a control packet for flow specification at the higher layer [165]. Moreover, the information of delay requirements must to be exchanged with the controller, which will incur extra traffic overhead [135].

$\mathrm{Na}$ et al. [110] proposed GTS Scheduling Algorithm (GSA) that tries to optimise GTS allocation based on the payload size and the delay constraint for data transmissions. Specifically, GSA checks schedulability of the requested requirements by estimating the delay of each transaction and analysing the relationship in between the delay and the number of allocated GTSs. Based on that, GSA tries to spread out the payload through several superframe by allocating the minimum number of GTSs to each transaction within each superframe. In this way GSA can ensure a smooth traffic flow between the coordinator and sensor nodes. However, this algorithm needs to exchange delay requirements between sensor nodes and the coordinator which increase traffic overhead [135].

Shrestha et al. [135] proposed a new scheme to prioritise GTS allocation 
based on the application data rate of each sensor node. It tries to utilise the radio bandwidth in the GTS in an optimal manner. In this algorithm, if the GTS request is unsuccessful, a sensor node needs to wait until the next superframe and sends the request again. Due to this delay, the sensor node might discard the data packet if its waiting time exceeds the delay limit. Moreover, the authors did not provide a detailed priority differentiation mechanism.

Huang et al. [64] proposed Adaptive GTS Allocation (AGA) which utilises the idea of assigning priorities in a dynamic fashion based on recent GTS usage feedbacks to provide low latency and fairness. Particularly, AGA is divided into two phases: (1) The classification phase, that assigns priorities to devices based on recent GTS usage feedbacks in a multilevel AdditiveIncrease/Multiplicative-Decrease (AIMD) manner. They suggest that recent GTS usage feedbacks give long-term transmission characteristics. (2) The scheduling phase, that allocates GTSs based on given priorities. However, the AGA scheme suffers firm some implementation overhead since extra information for devices must be recorded to allocate GTS resources. Besides, channel condition can play an important role in balancing traffic between CFP and CAP and none of the mentioned solutions has taken it into account [164].

To address IEEE 802.15.4 limitations in both CSMA/CA and GTS, crosslayer control provides a promising new direction for research [83, 49]. In a cross-layer approach, information from different layers of the protocol stack is collaboratively utilised to enhance the effectiveness of MAC layer. Soft computing techniques, in particular fuzzy logic, have been popularly used in cross-layering systems [120, 107, 31].

\subsubsection{Cross-layer Fuzzy Logic MAC}

FLCs have become one of the major control techniques because of their capability of solving previously intractable or very difficult control problems and their successful real-life applications. Due to their capability for 
dealing with nonlinear systems with multiple inputs and defining some rules to connect these inputs with the outputs, FLCs have been vastly used in cross-layering technologies such as localisation in WSNs [117], modelling and analysing the lifetime of WSNs [137], clustering in WSNs [101], event detection [94], coding scheme [136] and deployment in WSNs [176].

Mouzehkesh et al. $[107,108]$ proposed Dynamic delayed Medium Access Control ( $\mathrm{D}^{2} \mathrm{MAC}$ ) to improve the network reliability and performance of IEEE 802.15.4. They have introduced FuzzyMAxDelay to decide the upper limit of backoff delay instead of exponential backoff delay (see (2.3)). The backoff delay is calculated as follows:

$$
\begin{aligned}
& \text { backoffTime }=\operatorname{rnd}(0, \text { FuzzyMAxDelay }) \times B P, \\
& \text { where } 8 \leqslant \text { FuzzyMAxDelay } \leqslant 127
\end{aligned}
$$

where FuzzyMAxDelay is calculated by a FLC based on sensor node's data rate and the rate of successful channel access calculated as follows:

$$
\text { Channel }_{\text {clear }} \text { Rate }=\frac{\sum_{i=0}^{n} C C A_{2}}{n}
$$

where $C C A_{2}$ is the second successful CCA in IEEE 802.15.4 and $n(=20)$ is the number of superframes. $\mathrm{D}^{2} \mathrm{MAC}$ calculates Channel $_{\text {clear }}$ Rate for every $n$ superframes.

In $\mathrm{D}^{2} \mathrm{MAC}$, as the channel becomes busy, FuzzyMaxDelay will increase accordingly. Although, $\mathrm{D}^{2} \mathrm{MAC}$ managed to show improvements over IEEE 802.15.4 in term of reliability, it achieves this reliability by sacrificing packet delays.

A similar work was also conducted by Chen et al. in [31]. They proposed a soft-computing technique to improve both network reliability and performance. In their algorithm, a FLC is employed to calculate: (1) the upper limit of backoff delay by adding FuzzyBEField to the exponential backoff value and (2) the increase step size of NB in IEEE 802.15.4 through 
FuzzyNBStep respectively as follows:

$$
\begin{gathered}
\text { backoffTime }=\operatorname{rnd}\left(0,2^{B E}-1+\text { FuzzyBEField }\right) \times B P, \\
\text { where } 8 \leqslant \text { FuzzyBEField } \leqslant 127 \\
N B=N B+\text { FuzzyNBStep, where } 0<\text { FuzzyNBStep } \leqslant 4
\end{gathered}
$$

where both FuzzyBEField and FuzzyNBStep are produced by the FLC based on data rate of each sensor node and Channel $_{\text {clear }}$ Rate in (2.17). In contrast with $D^{2} \mathrm{MAC}$, the authors in [31] did not change the structure of exponential backoff algorithm. Furthermore, they tried to control packet delay by controlling NB. However, they still impose long packet delay to improve the reliability of the network, which is undesirable for time-sensitive applications in WBANs.

Although these approaches have improved IEEE 802.15.4 significantly, they have not taken GTS allocations into account. To address this limitation, Jing et al. [177] proposed Fuzzy Control Medium Access (FCMA) to prioritise GTS allocation and to control backoff delay in sensor nodes. Similar to $\mathrm{D}^{2} \mathrm{MAC}, \mathrm{FCMA}$ calculates the upper limit of backoff delay, which is $C W$, in IEEE 802.15.4. FCMA also changes the structure of IEEE 802.15.4 to accommodate more than seven GTS slots and increase channel utilisation. They claimed that they improved both reliability and performance of WBANs. However, their proposed method is centralised and even $C W$ size is controlled by the coordinator, which causes traffic overhead. Moreover, FCMA does not take channel condition into account, which might have negative impact on the reliability and performance of the network.

\subsubsection{Evolutionary design of Fuzzy Logic Controllers}

A major problem for FLCs is that their design is crucial for the cross-layer approaches discussed in Subsection 2.2.2 to be effective. In designing FLCs, we need to specify fuzzy set membership, the fuzzy operators and the Knowledge Base. However, a review of the literature suggests that this 
important design issue in WBANs has not been fully addressed. Very often, researchers rely purely on manual design of FLCs that requires huge human efforts, yet cannot provide any performance guarantees [120, 107, 31, 113].

Various automatic techniques have been utilised to design FLCs such as the table lookup schemes [159], gradient descent [159], clustering and gradient descent [168], and EC techniques [96, 23, 52, 59, 47]. In comparison with other techniques, EC techniques enjoy a clear advantage since they are capable of automatically designing different types of fuzzy logic rules, various forms of MFs, and the structure of rules base [45]. Moreover, EC techniques are gradient-free optimisation techniques [45] that are widely applicable specifically on noisy and multimodal problems. Because the design problem in WBANs can be generally considered to be noisy and multimodal [131], we believe EC techniques are suitable for our design problem.

In the literature, many EC techniques, including Genetic Algorithm (GA) $[23,84]$, PSO [17, 11, 111, 18] and DE [124, 59, 138, 118], have become popular tools for designing FLCs. Some representative and recent research works have been summarised in Table 2.1. For example, Bouarroudj et al. [19] successfully designed Gaussian MFs through PSO. Pishkenari et al. [124] used both GA and DE to design trapezoidal MFs of a FLC for controlling a rescue robot and showed that DE can outperform GA. Similarly, Yun et al. [173] used GA to design FLC for localisation in WSNs. Martinez et al. [99] also proposed designing MFs by using two popular EC techniques, i.e. GA and PSO. They tested their solution on a benchmark problem and suggested that PSO can converge more quickly than GA.

The design complexity to a large extent can also be affected by the coding scheme and its dimension in use. Bingül et al. [17] successfully designed Gaussian MFs through PSO for two controllers for a planar robot. They proposed a coding scheme which is divided into two parts. The first part is to encode the centre and deviation of the Gaussian MFs for the first controller and the second part is to encode parameters of MFs for the 
Table 2.1: Related works of EA-based FLCs design.

\begin{tabular}{|c|c|c|c|c|}
\hline & EA & Type of MF & Tuning Parameters & FLC Application \\
\hline Bingül et al. [17] & PSO & Gaussian & Centre and deviation & Planar robot \\
\hline Kim et al. [84] & GA & Triangular & $\begin{array}{l}\text { Three control } \\
\text { parameters of a } \\
\text { triangular MF }\end{array}$ & $\begin{array}{l}\text { OPS and FNC } \\
\text { for the inverted } \\
\text { pendulum }\end{array}$ \\
\hline Hachicha et al. [59] & $\mathrm{DE}$ & Triangular & $\begin{array}{l}\text { Three control } \\
\text { parameters of a } \\
\text { triangular MF }\end{array}$ & $\begin{array}{l}\text { Financial market } \\
\text { dynamics }\end{array}$ \\
\hline Nasseer et al. [11] & PSO & Triangular & $\begin{array}{l}\text { Centre and width } \\
\text { (Symmetric triangular) }\end{array}$ & $\begin{array}{l}\text { Speed of wind } \\
\text { turbines }\end{array}$ \\
\hline Chang et al. [32] & PSO & Triangular & $\begin{array}{l}\text { Centre value } \\
\text { (Symmetric triangular) }\end{array}$ & $\begin{array}{l}\text { Two wheeled } \\
\text { mobile robot }\end{array}$ \\
\hline Pishkenari et al. [124] & $\begin{array}{l}\text { GA } \\
\text { vs DE }\end{array}$ & Trapezoidal & $\begin{array}{l}\text { Four control } \\
\text { parameters }\end{array}$ & Rescue robot \\
\hline Martinez et al. [99] & $\begin{array}{l}\text { GA } \\
\text { vs PSO }\end{array}$ & $\begin{array}{l}\text { Combination of } \\
\text { Gaussian and } \\
\text { triangular }\end{array}$ & $\begin{array}{l}\text { Three control } \\
\text { parameters of a } \\
\text { triangular MF, } \\
\text { Centre and } \\
\text { deviation } \\
\text { Gaussian MF }\end{array}$ & $\begin{array}{l}\text { Benchmark } \\
\text { problem }\end{array}$ \\
\hline Bouarroudj et al. [19] & $\begin{array}{l}\text { PSO } \\
\text { vs GA }\end{array}$ & Gaussian & Centre and deviation & $\begin{array}{l}\text { Maximum power } \\
\text { point tracking } \\
\text { of photovoltaic } \\
\text { system }\end{array}$ \\
\hline Yun et al. [173] & GA & Trapezoidal & $\begin{array}{l}\text { Four control } \\
\text { parameters }\end{array}$ & $\begin{array}{l}\text { Localisation in } \\
\text { WSNs }\end{array}$ \\
\hline Liu et al. [96] & GA & Triangular & Linguistic Hedge & $\begin{array}{l}\text { Cart-pole balance } \\
\text { system }\end{array}$ \\
\hline Casillas et al. [23] & GA & Triangular & $\begin{array}{l}\text { Linguistic Hedge } \\
\text { and Three } \\
\text { control parameters } \\
\text { of a triangular MF }\end{array}$ & $\begin{array}{l}\text { The rice and } \\
\text { electrical } \\
\text { benchmark } \\
\text { problems }\end{array}$ \\
\hline
\end{tabular}


second controller. Hachicha et al. [59] utilised DE to design triangular MFs to model the financial market dynamics. They proposed a two part coding scheme which encodes centre and linguistic of each MF respectively. Furthermore, Nasseer et al. [11] focused on reducing the search dimension in designing a FLC through PSO for wind turbine. To reduce the search dimension, the authors used triangular MFs with only two control parameters, i.e. centre and width. Similarly, Chang et al. [32] used symmetric triangular MFs to further reduce the search space.

Liu et al. [96] studied a similar problem and suggested only searching for a collection of linguistic modifiers with respect to a fixed collection of fuzzy rules for efficient design of FLCs. These linguistic modifiers are utilised to dynamically tune the shape of MFs and increase the convergence. Following this work, Casillas et al. [23] suggested a deep structure for designing FLC, which uses both the linguistic modifiers and the control parameters of MFs. They studied this deep structure and claimed that the linguistic modifiers will not change the overall structure of FLCs and hence maintain their interpretability. Accordingly, the authors proposed a three fold coding scheme for their proposed Fuzzy Rule-Based Systems (FRBSs) genetic tuning. The first part is 3-tuple of real values for each triangular MF. The second and third part are to encode linguistic modifiers. Although, these research works [23, 124, 17, 59, 32, 96] have focused on examining domain-specific coding schemes, there is a lack of comparison between different coding schemes in terms of their flexibility, effectiveness and interpretability.

The effectiveness of EC techniques in designing FLCs has been widely demonstrated. However, they could easily become unaffordable in practice due to (1) high computational cost and (2) the curse of dimensionality. EC techniques mostly must evaluate a large number of candidate solutions before a satisfying result can be achieved. The evaluation can be a major source of high computational cost. Moreover, increasing the number of input and output variables in FLCs results in the curse of dimensional- 
ity. Therefore, in this thesis, we study surrogate models and cooperative coevolutionary techniques to address these issues, respectively.

\section{Surrogate-assisted EC techniques}

The major computational cost for designing FLC in WBANs comes from evaluating the fitness function. An effective way to address this issue is to use surrogate models of fitness functions which have been used widely in many optimisation problems such as aerodynamic design [127] and circuit design [142]. For example, Praveen et al. [127] utilised a Radial Basis Function (RBF) based surrogate to assist PSO in optimising aerodynamic shapes. A new system called Design and Analysis of Computer Experiments (DACE) [122] has been also exploited to build a surrogate model to reduce the computation cost of EAs in standard benchmarking problems and a simulation-based hydrology application. Jin et al. [76] utilised feed-forward MultiLayer Perceptrons (MLP) to build a surrogate model for optimisation problem such as aerodynamic design. A comprehensive survey of different surrogate related research is provided in [74].

To build an effective and efficient surrogate model, two requirements should be addressed [75]: (1) accuracy of the model and (2) selection of candidate solutions for re-evaluations. The accuracy of surrogate models is essential, because an inaccurate model can result in incorrect convergence. To improve the accuracy of surrogate models both off-line $[67,77]$ and online training $[74,76,127,129]$ can be used. The former focuses on training the model before using EC and the latter updates the model during the evolutionary design process. It has been shown that, because of the lack of data and the high dimensionality of many problems, online training can perform better than off-line training.

In the online training, surrogate models and real fitness functions have been mostly used together to improve the accuracy of surrogate models $[74,76,127,129]$. This cooperation can improve the surrogate model during the evolutionary process by re-training the model with respect to new 
samples. In other words, in every generation some candidate solutions are selected and re-evaluated with the real fitness function. The new reevaluated candidate solutions are added to the data set to re-build a more accurate model. Nevertheless, building the surrogate model from scratch in every generation might be inefficient $[21,97]$. Therefore, many research efforts have utilised online learning to improve the accuracy of the model with respect to new samples during evolutionary process.

The second requirement to build an effective and efficient surrogate model is to choose the candidate solutions for re-evaluations. Several strategies have been suggested to select candidate solution for re-evaluations. For example, some research efforts have tried to select the candidate solution for re-evaluation by clustering the populations. They select the candidate solution that is closest to each cluster centre $[55,105]$ or the fittest in the cluster $[55,56]$. The most straightforward and popular strategy is to evaluate the number of candidate solutions with higher fitness in the population $[75,74,127,129,77]$.

\section{Coevolutionary design of fuzzy logic control}

Extending the number of input and output variables in FLCs results in increased difficulty of designing FLCs. To address this issue, a divide-andconquer strategy could be a natural approach to tackle this complexity. For example, Potter and Jong [126] proposed a Cooperative Co-Evolutionary Algorithm (CCEA) to cope with high complexity in optimisation problems. In their solution, a problem is decomposed into several smaller sub-components, each of which can be evolved through a separate GA sub-population. While each sub-population is evolved, the remaining subpopulations can be held fixed. Recently CCEAs have been applied to many optimisation problems [126, 143, 133, 69, 156, 92]. For example, Stonier and Young [143] used CCEAs to optimise a hierarchical fuzzy controller for the inverted pendulum. Roberts et al. [133] utilised Cooperative Coevolutionary Genetic Algorithm (CCGA) for image feature construction and 
object detection. Ibáñez et al. [69] proposed a cooperative coevolutionary approach in craniofacial superimposition which deals with the use of imprecise cephalometric landmarks in the skullface overlay process.

Bergh and Engelbrecht [156] proposed Cooperative Particle Swarm Optimiser (CPSO) that can perform much better than the original PSO on several benchmark optimisation problems. This is achieved by using multiple sub-populations to optimise different components of the solution vector cooperatively. $\mathrm{Li}$ and Yao proposed [92] Cooperative Coevolving PSO (CCPSO), which is an improvement over CPSO, to tackle high dimensional non-separable optimisation problems. They used random grouping and adaptive weighting to handle high dimensional non-separable problems. $\mathrm{Li}$ and Yao [93] also tried to improve CPSO further by proposing CCPSO, which enhanced the likelihood of optimum grouping by increasing the frequency of random grouping and introducing the dynamic subcomponent sizes for random grouping.

The most straightforward method to construct a candidate solution as a whole is to make it interact with all possible collaborators from the other sub-populations. This method, which is also called complete pairwise interaction, is highly computationally expensive [161]. An alternative solution is to select a limited number of collaborators from other sub-populations. Choosing collaborators is connected to problem characteristics and could be among the most important design decisions for the successful application of CCEAs [161]. Specifically, CCEAs evaluate the fitness function based on how well the candidate solution cooperates with the other candidate solutions with which it interacts. Candidate solutions succeed when they find collaborators effectively.

Commonly researchers have chosen the best fit solution from alternative sub-populations $[69,92,161]$. However, this method could be greedy and CCEAs could fall into local optima $[156,161]$. Therefore, some researchers have selected an alternative method which involves two candidate solutions, i.e. the best and a random candidate solution [92, 161]. In this 
method, both selected collaborators are evaluated with the current candidate solution and the higher fitness determines the fitness of the current candidate solution $[156,161]$. Although these methods are commonly used by researchers, they are designed to tackle general optimisation problems. We believe that, considering the unique characteristic of design problems can improve the effectiveness of collaborator selections.

\subsection{Chapter Summary}

This chapter presented important technical background. First, it provided an introduction to Media Access Control (MAC) protocols in Wireless Body Area Networks (WBANs) and IEEE 802.15.4 standard. Then, it over-viewed Fuzzy Logic Controls (FLCs) and Evolutionary Computation (EC) techniques. This chapter also reviewed and discussed works related to MAC protocols and cross-layer FLC approaches to improve the quality of communication in WBANs. Next, it reviewed evolutionary approaches to effectively design FLCs.

This chapter also discussed the limitations of the existing work which form the motivations of this research. The research aims to utilise crosslayer FLCs to improve reliability and performance of MAC protocols in WBANs. However, the works in the literature mostly rely on a manual approach to design FLCs, which is laborious and error-prone. Despite huge human efforts, the resulting FLCs may have poor quality in practice. Consequently, this research aims to address this limitation by introducing an evolutionary approach to design FLCs in the context of WBANs. To achieve these aims we need to address the limitations of existing work as follows:

- Some existing research efforts have already utilised FLCs to improve the reliability and performance in WBANs [119, 107, 31]. However, we believe that they have not used FLCs in an effective manner. They have mostly achieved high levels of reliability and performance at the 
cost of significantly increasing the packet delay [113]. As a matter of fact, the average packet delay must be kept at a reasonably low level in time-critical WBAN applications.

- Existing research works mostly have employed FLCs for only sensor level MAC [107, 115, 31]. However, it hs been shown that using the coordinator level control could also improve the reliability and performance of the network $[135,64]$. Although, some research efforts have been performed to optimise the coordinator-level bandwidth allocation to sensor nodes, few have exploited the advantages of using both distributed and centralised communication methods. There is a need for an effective multilevel cross-layer FLC and a balance between these levels to significantly enhance reliability and performance in the context of WBANs.

- It has been shown that FLCs have potential to improve the communication quality. However, the effectiveness of FLCs depends on their design. Nevertheless, a review of the literature suggests that this important design issue in WBANs has not been fully addressed. Very often, researchers rely purely on manual design of FLCs that requires huge human efforts, yet cannot provide any performance guarantees [120,107, 31, 113]. It also been shown that EC techniques are desirable to be used to design FLCs [159, 168, 96, 23, 52, 59, 47]. In order to design FLCs in the context of WBANs, we need to address the following requirements:

- Encoding the candidate solutions: In the literature, most of the research works only have studied domain-specific coding schemes and some have studied general coding schemes. However, it is not clear which coding scheme is particularly useful for our design problem. Thus, it is necessary to discover which coding scheme can be effective in our design approach. 
- Fitness function: There is a need to design a meaningful fitness function to guide the evolutionary design process. Our main design goal is to optimise the reliability of WBANs. On the other hand, the performance of the network has to be competitive to IEEE 802.15.4. However, increasing reliability may lead to an increase in packet delay. Consequently, reliability should be constrained to packet delay.

- Fitness evaluation method: To the best of our knowledge, there is no evaluation method in the literature review for different WBAN's settings. Therefore, to improve the general applicability of our approach, we need to define the fitness evaluation method based on different WBAN's settings.

- Efficiency of design process: Since evaluating the fitness of any candidate FLC design is time-consuming, which has impact on the practicality of the design approach, the efficiency of the design process has to be improved. Therefore, we need to rely on surrogate models to boost the efficiency of the design process.

- Utilising cooperative coevolutionary computation techniques could be a promising and suitable solution to effectively and cooperatively design multilevel FLCs. However, one major challenge is collaborator selection methods which can be crucial in the design process. Many research works recommend to choose the best fit or random solution from alternative sub-populations [126, 156, 161]. However, these methods are designed to tackle general optimisation problems and may not be effective enough in our specific design problem.

The following three chapters of this thesis will address and discuss the above mentioned issues. 


\section{Chapter 3}

\section{Cross-Layer Fuzzy Logic based Backoff}

\subsection{Introduction}

Past research has evaluated the efficiency of commonly used IEEE 802.15.4 in WBANs [30,3], showing that this standard can be very limited in terms of its reliability (measured by PDR), and performance (measured by throughput) $[120,53,24,113]$.

In practice, reliability is an important requirement. For instance, in healthcare monitoring applications, reliable communication is essential in order to bring dependable medical care to patients. Since WBANs are used to carry medical data and signal emergencies like vital organ failures, in worst-case scenarios, communication failure may cause death when a lifethreatening event has been left unattended. Driven by this understanding, some exciting research has already been conducted to enhance the level of PDR $[119,107,31]$. Accordingly, the aim of this chapter is to improve the reliability of the current standard.

In addition to reliability, performance in terms of throughput poses another issue for IEEE 802.15.4 [119]. Particularly for the healthcare monitoring applications, WBANs are required to achieve high throughput in order 
to support sensors with high transmission rate such ECG sensors. For such applications to be successful, Chen et al. [31] proposed a soft-computing technique to improve both network reliability and performance. A similar work was also conducted by Mouzehkesh et al. [107] by proposing Dynamic delayed Medium Access Control ( $\mathrm{D}^{2} \mathrm{MAC}$ ) to improve the network reliability and performance of IEEE 802.15.4.

However, reliability and performance improvement should not be realised at the cost of significantly increasing the packet delay. As a matter of fact, the average packet delay must be kept at a reasonably low level. This requirement can be easily understood in medical applications where timely communication is highly desirable. In order to decrease the network delay, Additional Carrier Sensing (ACS) method [163] has been proposed to use the third Channel Clear Assessment (CCA) to reduce unnecessary delay introduced by the shifted backoff period.

In Carrier Sense Multiple Access with Collision Avoidance (CSMA/CA), the Collision Avoidance (CA) mechanism is achieved by introducing a random backoff delay before assessing the channel through carrier sensing. Particularly, when the channel is assessed as busy, the maximum backoff delay will be increased at an exponential speed. For each new transmission, CSMA/CA in IEEE 802.15.4 ignores the most recent channel condition. It blindly restarts the whole backoff process instead of adjusting backoff delay based on the recent channel condition. As a result, while the channel is still busy, the collision rate might increase and accordingly throughput would decline. Different from this standard approach, in this chapter, backoff delay is dynamically adjusted through a new cross-layer approach. The adjustment is undertaken by a fuzzy logic system designed by us. We have also conducted a manual process to fine-tune fuzzy rules for the purpose of making it more suitable for healthcare applications. 


\subsubsection{Chapter Goals}

This chapter targets the problem of inflexible backoff delays in IEEE 802.15.4. This chapter aims to address this issue by proposing a new cross-layer approach to adaptively produce backoff delays. This approach utilises soft computing techniques, in particular fuzzy logic, which have been popularly used in cross-layering systems [120, 107, 31]. We demonstrate the effectiveness of using FLCs for adaptively producing backoff delays to improve reliability and performance in WBANs. The following are the chapter goals briefly:

1. Extending IEEE 802.15.4 to facilitate fuzzy logic based MAC and make it more competent for various WBAN-based applications.

2. Manually designing the FLC used to improve reliability and performance of WBANs.

3. Preserving backward compatibility with IEEE 802.15 .4 by using FLC and not changing the fundamental structure of the IEEE 802.15.4.

4. Finally, experimentally demonstrating the potential usefulness of the new scheme with comparison to several recently proposed algorithms such as CSMA/CA in IEEE 802.15.4, ACS [163], D²MAC [108] and a recent algorithm proposed by Chen et al. [31]. For the sake of simplicity, in this research, "IEEE 802.15.4" refers to the standard CSMA/CA algorithm.

\subsubsection{Chapter Organisation}

The remainder of this chapter is organised as follows. Section 3.2 proposes the cross-layer fuzzy logic based backoff algorithm. Section 3.3 presents manual FLC design process. Section 3.4 describes the simulation implementation and results in detail. Finally, we conclude this chapter in section 3.5 . 


\subsection{A Cross-Layer Backoff Mechanism Using Fu- zzy Logic Controller}

Many research work show that the quality of wireless channels near the human body is very poor and path loss is very high [131]. The poor channel condition in WBANs may result in transmission failures and decreased reliability and performance which are the major concerns especially in healthcare applications [113]. Moreover, IEEE 802.15.4 does not consider application-specific requirements, such as frequency of channel access, when adjusting the backoff delay. Moreover, whenever the channel is assessed as busy, IEEE 802.15.4 will blindly increase BE incrementally. The work flow in Figure 3.1 implies that every node will always restart the whole backoff process for each new transmission, regardless of applicationspecific requirements and recent channel condition. Consequently, nodes might fail to adjust the backoff delay sufficiently quickly and this would significantly increase the collision rate in WBANs. Accordingly, reliability and channel utilisation are negatively affected.

In order to tackle this issue, we have introduced Cross-Layer Fuzzy Logic based Backoff (CLFB) [113] to adapt backoff delay by adjusting Backoff Exponent (BE) based on different channel conditions and application requirements as shown in Figure 3.2. Figure 3.1 illustrates both slotted CSMA/CA and CLFB. Whenever the fuzzy Enable=true, CLFB will be used for determining backoff delay. Otherwise, the standard slotted CSMA/CA in IEEE 802.15.4 will be adopted. CLFB uses a FLC to adaptively tune $\mathrm{BE}$ depending on both recent network conditions and application requirements.

To distinguish BE determined by FLC in CLFB from BE in IEEE 802.15.4, the former will be called fuzzyBE in this research. Consequently, the random backoff delay followed by each node before accessing the channel in CLFB 


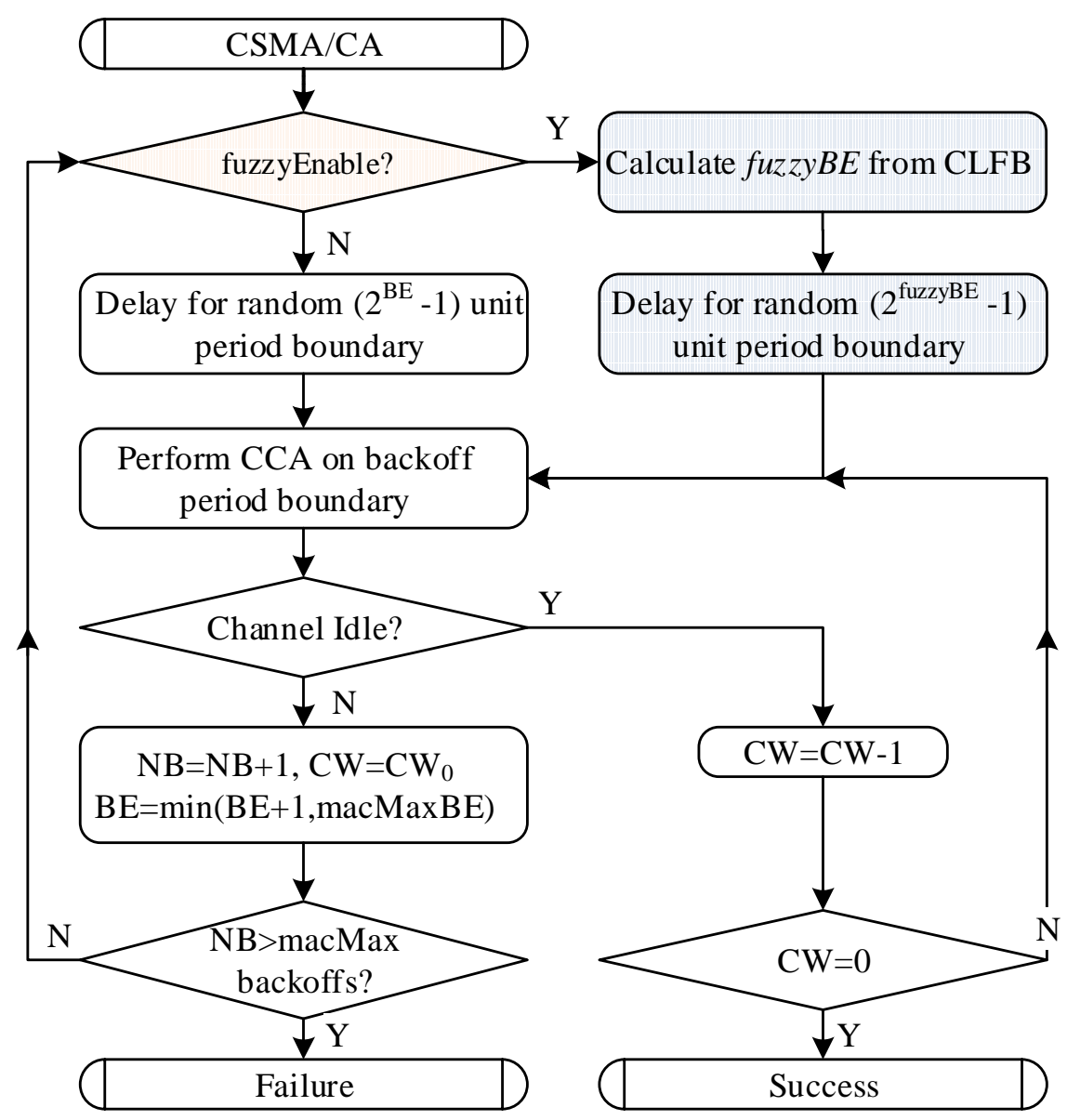

Figure 3.1: The flow chart of CSMA/CA and CLFB algorithms where differences between the standard CSMA/CA and Cross-Layer Fuzzy logic Backoff (CLFB) are highlighted. 


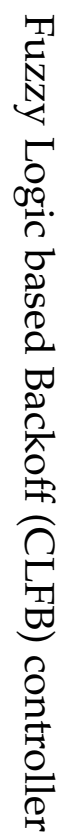

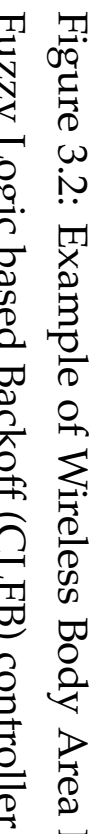

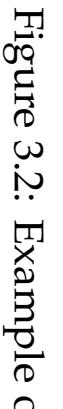

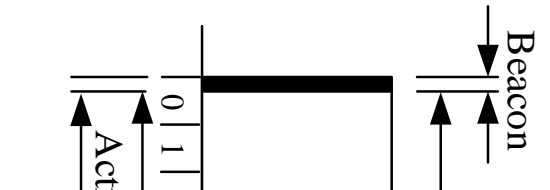

$41 ㅇ$

$\frac{D}{0}$

2

$\sum_{0}^{+}$

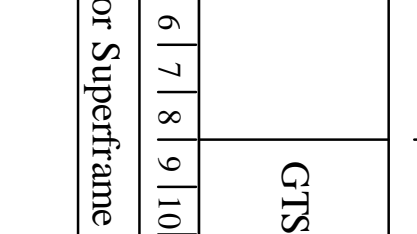

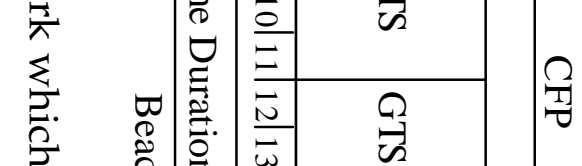

覀

界

TI

$\stackrel{\infty}{\stackrel{0}{N}}$

जั

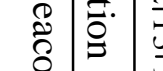

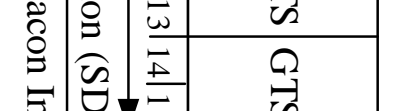

क

$\checkmark$

24

$+$

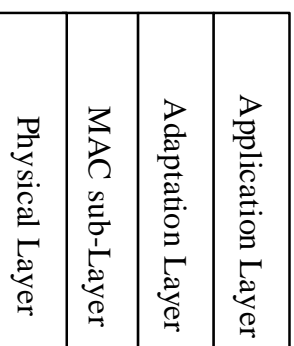

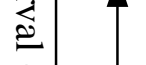

ש़

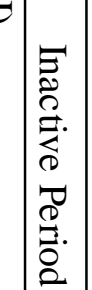

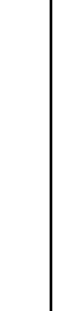

हี

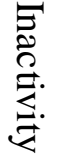

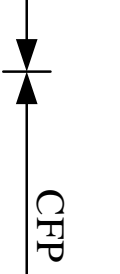

$\gamma$

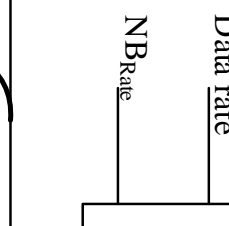

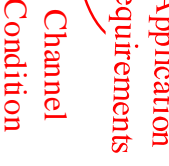

告

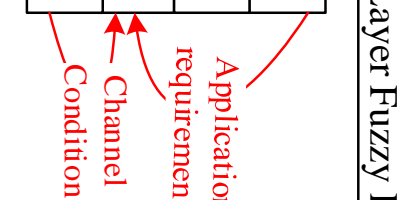

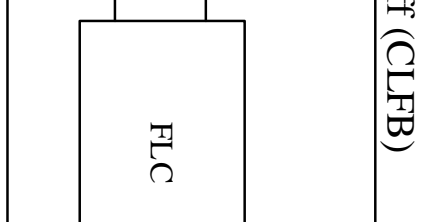

च

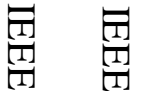

용

ur ur

in

क

1
3
0
0
0
0
0
0
4
4
0
0
0
0
0
2
0
0

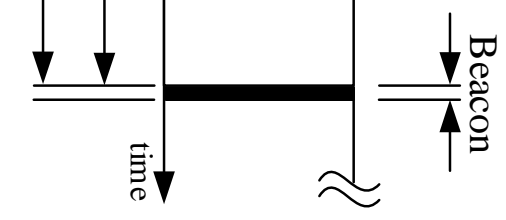


is obtained as below:

$$
\begin{aligned}
\text { backoffTime }=\operatorname{rnd}\left(0,2^{\text {fuzzyBE}}-1\right) \times & B P, \\
& \text { where } f u z z y B E \in\{2, . ., 8\}
\end{aligned}
$$

As illustrated in Figure 3.3, the FLC in CLFB accepts two input variables, i.e. $N B_{\text {Rate }}$ and data rate, which are described in more detail in Subsection 3.2.1. Moreover, it produces fuzzyBE as an output. Standard Mamdani fuzzy system will be used for inferencing in our FLC [100].

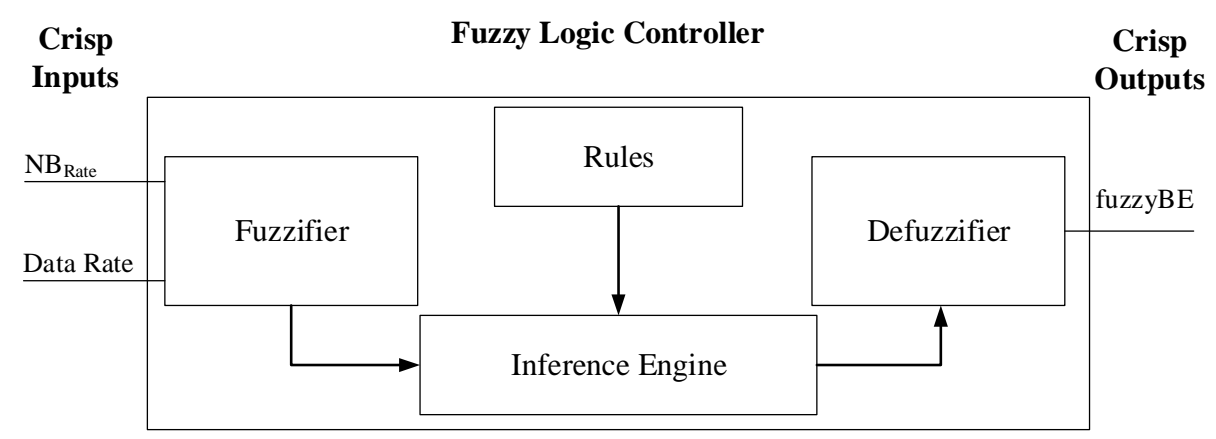

Figure 3.3: Architecture of fuzzy logic controller for CLFB.

\subsubsection{Fuzzy Input and Output Variables}

The first input variable of the FLC in CLFB is $\mathbf{N B}_{\text {Rate }} . \mathrm{NB}_{\text {Rate }}$ is measured through the moving average of NB value over time as below:

$N B_{\text {Rate }}(t+1)= \begin{cases}\delta \times N B+(1-\delta) \times N B_{\text {Rate }}(t), & \text { if } N B \leq \text { macMaxBackoffs } \\ \delta \times N B_{\text {penalty }}+(1-\delta) \times N B_{\text {Rate }}(t), & \text { if } N B>\text { macMaxBackoffs }\end{cases}$

where $\delta, 0 \leqslant \delta \leqslant 1$, is a discount factor and $N B_{\text {penalty }}$ is a penalty for every transmission failure. In particular, NB will increase whenever the channel is in use. When it exceeds macMaxBackof $f s$, the corresponding transmission effort will fail. Consequently, $N B_{\text {penalty }}$ must be strictly higher than macMaxBackoffs. 
Since NB serves as a direct indication of the channel condition in the recent past, whenever channel condition deteriorates, $\mathrm{NB}_{\text {Rate }}$ starts to increase quickly. Similarly, it decreases when the channel condition is improved. The $\mathrm{NB}_{\text {Rate }}$ in our FLC is categorised into four fuzzy levels, i.e. low, medium, medium-high and high. We choose to use four different fuzzy levels because key related research $[119,107,31]$ prefers to use at most four fuzzy levels for any types of input to a fuzzy system. However, this is not a technical restriction. In the future, we will study the possible benefits of using more fine-grained fuzzy levels. In the calculation of $\mathrm{NB}_{\text {Rate }}$ (Eq. 3.2), a penalty will also be applied. Specifically, when a transmission attempt fails because NB exceeds macMaxBackof $f s$, our algorithm introduces the penalty to clearly distinguish between failures during CCAs and a complete transmission failure. The penalty is represented by the NB value of six, which is clearly higher than the maximum possible NB value during any CCAs. Due to this penalty method, the full possible range of NB-rate is from zero to six.

The second input variable to our FLC in CLFB is data-rate. It is an important variable since it helps to balance between the waiting time and the channel condition. To cope with various network arrangements, we decided to normalise the data-rate. Upon joining the WBAN, a sensor node will send its application data-rate to the coordinator, which will then determine the maximum data-rate among all individual sensors in the network. The coordinator will subsequently inform the whole network about the maximum data-rate. Each sensor node will normalise its own data-rate within the range of 1 to 100 accordingly. One potential benefit of using normalised data-rate is to improve the utilisation of the network capacity. The normalised data rate is further partitioned into four different fuzzy levels, i.e. low, medium, medium-high and high.

The output from our FLC is the fuzzyBE. In order to have a reasonable level of accuracy, fuzzyBE is decomposed into four separate fuzzy levels, which are $B 1, B 2, B 3$ and $B 4$. The centre of gravity is used in our FLC to 
defuzzify fuzzyBE and produce a crisp value in (3.1).

For simplicity and efficiency, following other researchers [107, 31], we use the triangular shaped membership function for all input and output variables. Examples of such membership functions are illustrated in Figure 3.4. In subsection 3.3, we will explain how the membership functions will be manually designed to improve reliability and performance of WBANs.

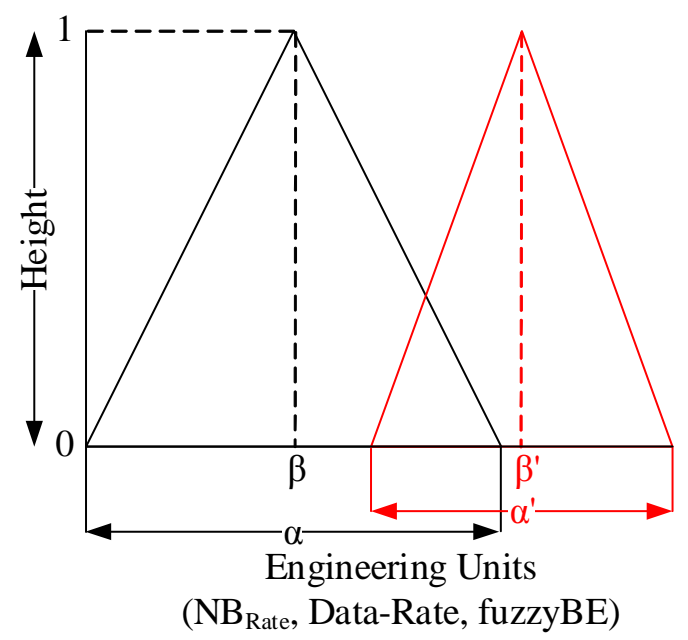

Figure 3.4: An example of triangular membership functions and tuning by adjusting the membership function parameters

\subsubsection{Fuzzy Logic Rules}

In this study, there are 16 different rule antecedents, (i.e. 4 fuzzy levels of $\mathrm{NB}_{\text {Rate }} \times 4$ fuzzy levels of data rate). Consequently, our FLC in CLFB comprises 16 separate fuzzy rules. Each rule follows a basic structure as shown below:

$$
\begin{aligned}
& R^{(n)}: \text { IF NB- } \text { Rate }_{n} \text { is }_{1}^{i} \\
& \text { and Data - Rate } \text { is }_{2}^{i} \\
& \text { THEN fuzzyBE } E_{n} \text { is } c^{i}
\end{aligned}
$$

A summary of all the fuzzy rules in CLFB is also presented in Table 3.1. 
According to this table, whenever $\mathrm{NB}_{\text {Rate }}$ is Low, meaning that channel is not busy, our FLC will suggest short delays by setting fuzzyBE to B1, e.g. fuzzy rules $\mathrm{R}^{(1)}, \mathrm{R}^{(2)}$ and $\mathrm{R}^{(3)}$ in Table 3.1. However, the node with High data rate tends to receive longer delays to prevent it from blocking low data rate nodes, e.g. $\mathrm{R}^{(4)}$. When the channel is more congested, i.e. $\mathrm{NB}_{\text {Rate }}$ is at a Medium level, our FLC will produce comparatively larger fuzzyBE, e.g. $\mathrm{R}^{(5)}, \mathrm{R}^{(6)}$ and $\mathrm{R}^{(7)}$. Meanwhile nodes with High data rates will receive even longer delays, e.g. $R^{(8)}$. This happens because the node with higher data rate produces more packets and tries to access the channel more frequently. By receiving a longer delay, the probability of packet collisions can be reduced.

Furthermore, if the channel becomes even more congested, i.e. MediumHigh, to reduce the chances of collision the backoff delays recommended by corresponding rules, i.e. $\mathrm{R}^{(9)}, \mathrm{R}^{(10)}$ and $\mathrm{R}^{(11)}$, will become B3. Moreover, the node with High data rate will have the longest delay, i.e. B4. Finally when channel usage is High, our FLC will produce the longest delay B4, regardless of the data rate input. The only exception is the nodes with Low data rate which will still receive a shorter delay, i.e. B3.

As an example for the above mentioned rules, when we have two low data rate sensor nodes in the network, the channel is idle most of the time. Consequently, based on $\mathrm{R}^{(1)}$, fuzzyBE will be short and every sensor node will experience less delay. On the other hand, when there are several high data rate sensor nodes in the network, the channel is mostly busy. According to $\mathrm{R}^{(12)}$ and $\mathrm{R}^{(16)}$, fuzzyBE will be sufficiently high to avoid unnecessary collisions for the overall reliability of WBANs.

\subsection{Manual Design of Fuzzy Logic Controllers}

In practice, the effectiveness of FLC depends heavily on its control rules and membership functions. Therefore, the tuning and adjustment of membership functions become an important part of FLC design. At this stage of 
Table 3.1: Fuzzy Logic rules for CLFB.

\begin{tabular}{|l|c|c|c|c|}
\hline \multirow{2}{*}{ NB $_{\text {Rate }}$} & \multicolumn{4}{|c|}{ Data Rate } \\
\cline { 2 - 5 } & Low & Medium & Medium High & High \\
\hline Low & $R^{(1)}: B 1$ & $R^{(2)}: B 1$ & $R^{(3)}: B 1$ & $R^{(4)}: B 2$ \\
\hline Medium & $R^{(5)}: B 2$ & $R^{(6)}: B 2$ & $R^{(7)}: B 2$ & $R^{(8)}: B 3$ \\
\hline Medium High & $R^{(9)}: B 3$ & $R^{(10)}: B 3$ & $R^{(11)}: B 3$ & $R^{(12)}: B 4$ \\
\hline High & $R^{(13)}: B 3$ & $R^{(14)}: B 4$ & $R^{(15)}: B 4$ & $R^{(16)}: B 4$ \\
\hline
\end{tabular}

the research, because the total rule set is reasonably small, we designed our FLC manually as explained below.

One of the most common ways of tuning membership functions is to adjust the basic parameters defining them. For this purpose, two tuning parameters, i.e. $\alpha$ and $\beta$ as shown in Fig. 3.4, are considered, . In fact, $\alpha$ controls the shortening and widening of the membership function and $\beta$ moves the membership functions to the left and right. The aim of tuning is to adjust the two parameters in a direction of improving WBAN reliability and performance.

In this research, we have three steps in the tuning process. They are detailed below,

1. Tuning the output membership functions: because the output membership functions have more influence on the performance of FLC, the adjustment is carried out on them first. Particularly the input membership functions are held steady while tuning the output membership functions. Upon adjusting a membership function, we first adjust parameter $\beta$. After each adjustment 30 independent evaluations are performed to confirm its usefulness, i.e. improving both reliability and performance of IEEE 802.15.4. After three local adjustments, we then proceed to adjust $\alpha$ by following an identical process. After changing $\alpha$ and $\beta$ individually, we have a good approximation of $\alpha$ and $\beta$. Then we use them to perform several steps of random local 
search to finally determine the values for $\alpha$ and $\beta$. This process is applied on all B1, B2, B3, and B4 membership functions.

2. Tuning the input membership functions: In order to investigate suitable settings for input membership function and further improve performance of FLC, the input membership functions are tuned and the output membership functions remain unchanged. For each membership function, tuning of $\alpha$ and $\beta$ follows basically the procedure described in step 1 .

3. Tuning both the input and output membership functions: the previous two steps follow a greedy search strategy to find out the suitable settings for each membership function. In this final step, in order to improve FLC performance further, a local search is performed to tune both inputs and output membership functions simultaneously.

By following this process, we finally determine the suitable membership functions for each fuzzy level of every input and output variable. These functions are presented in Fig. 3.5 to make the simulation results reproducible.

\subsection{Simulation Implementation and Results}

In order to evaluate the reliability and performance of CLFB and compare it with many competing algorithms in the literature, including IEEE 802.15.4, ACS, and $D^{2} \mathrm{MAC}$, as well as a recently proposed algorithm [31] termed "NB-Step", we have performed simulation study in this chapter. In this section, detailed information and discussion related to the simulation environment, including important simulation settings, will be described in subsection 3.4.1. The simulation results and discussions will be presented in subsection 3.4.2. 


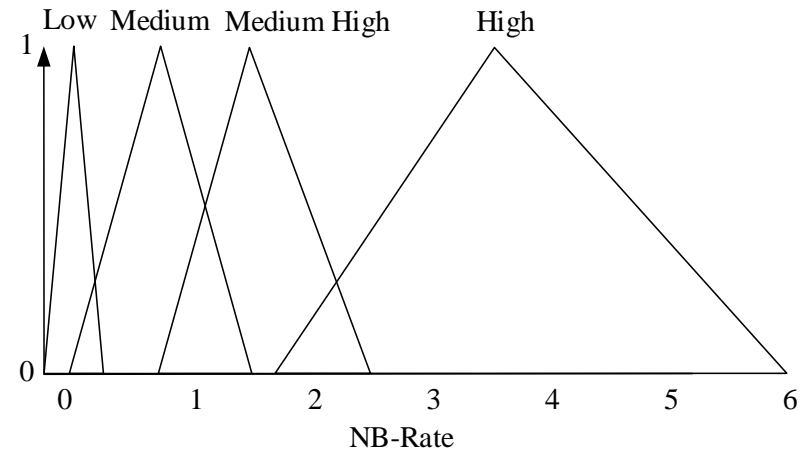

(a) $\mathrm{NB}_{\text {Rate }}$

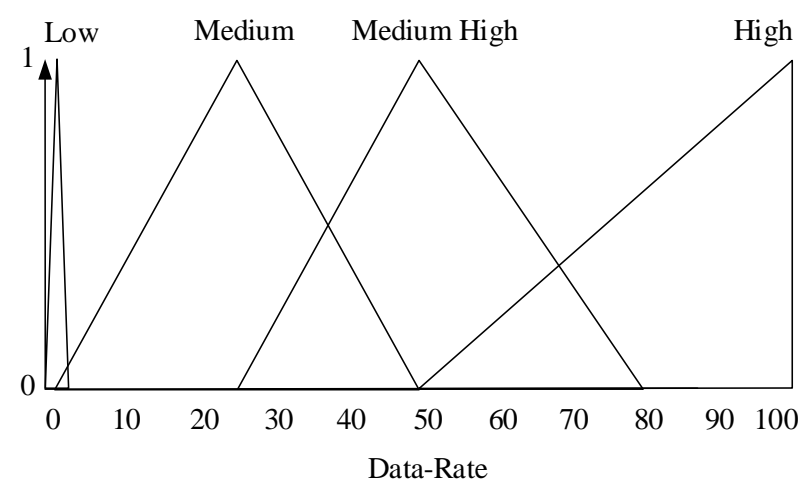

(b) Data-Rate

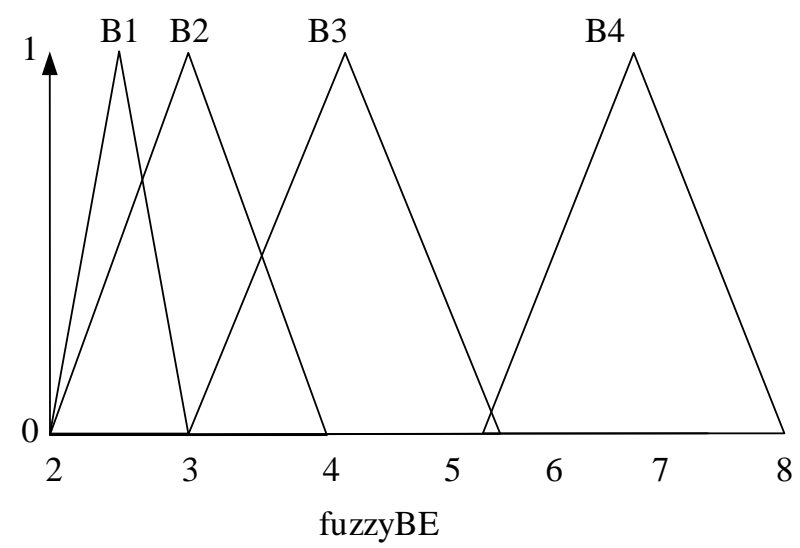

(c) fuzzyBE

Figure 3.5: The tuned membership functions 
Table 3.2: Communication specifications of Sensor nodes used in the simulation $[2,119]$.

\begin{tabular}{lccc}
\hline Sensor Node & ECG & Respiratory Rate & Motion Sensor \\
\hline $\begin{array}{l}\text { Traffic } \\
\text { Generation }\end{array}$ & Constant & Constant & Poisson \\
$\begin{array}{l}\text { Distribution } \\
\text { Data Rate }\end{array}$ & $156.25 \mathrm{Bps}$ & $15 \mathrm{Bps}$ & $64 \mathrm{Bps}$ \\
\hline \hline Sensor Node & Temperature & Heart Rate (HR) & Blood Pressure (BP) \\
\hline $\begin{array}{l}\text { Traffic } \\
\text { Generation }\end{array}$ & Poisson & Constant & Constant \\
Distribution & & & $512 \mathrm{Bps}$ \\
Data Rate & $12.5 \mathrm{Bps}$ & $20 \mathrm{Bps}$ & \\
\hline
\end{tabular}

\subsubsection{Simulation Environment}

In this research, we chose to use OMNeT++ 4.4 as our simulation tool [5]. $\mathrm{OMNeT}++$ is an open source network simulator framework with support for a wide variety of communication protocols. Our simulation is set up in a star-based WBAN with a single WBAN coordinator. To evaluate network conditions in different traffic loads, the number of sensors will increase ranging from two to nine. The simulation scenarios studied here are heterogeneous since we use up to six different types of medical sensors with different data rates. A summary of different sensors and their communication features can be found in Table 3.2 (i.e. 3-lead ECG, respiratory rate, blood pressure, heart rate, motion and temperature sensors). Although these sensors only represent a small portion of all available medical sensors, they are the most frequently used in many simulation studies [119]. To produce reliable results, each simulation scenario is evaluated independently 30 times. The averages of these runs will be presented as the simulation results. 
All body sensors are randomly placed in a $2 \times 2 \mathrm{~m}^{2}$ region with a WBAN coordinator in the centre. For simplicity, sensor nodes are initiated with the same amount of battery, i.e. $5500 \mathrm{mAh}$. Our simulation uses the IEEE 802.15.4 standard upper frequency band at $2.4 \mathrm{GHz}$, which is an important and commonly available frequency band in healthcare, with the standardised data rate of $250 \mathrm{~Kb} / \mathrm{s}$ and the maximum payload size of 102 bytes. The scenarios, which are considered by our simulation, depend on the frequency band of $2.4 \mathrm{GHz}$, which is suitable for body surface to body surface communication under two possible conditions, namely Line-of-Sight (LoS) propagation and Non-Line-of-Sight (NLoS) propagation.

In order to create a simulation environment that closely approximates real communication situations, we used the log-normal shadowing model [147] to build the channel model. Existing research suggests that, in WBANs, the log-normal shadowing model can better capture the smallscale fading than the traditional Rayleigh and Ricean distributions. The path loss exponent is set to three which is the exponent along the front of the human body. In this research, " $\delta$ " in (3.2) is set to 0.85 . This setting comes out of a trial and error process. We found that, by using it, NB-rate always presents a good indication of channel condition in our simulated WBAN.

\subsubsection{Simulation Results}

In this subsection, the results obtained from the simulation are presented. The metrics, which are utilised to assess the reliability and performance of the network, are:

- Packet Delivery Ratio (PDR): the ratio of the number of sent data packets to the number of delivered data packets as follow:

$$
P D R=\frac{\text { Number of Packets Received by Coordinator }}{\text { Total Number of Packets Sent by all Sensor Nodes }}
$$


- Collision Rate: the average number of data packet collisions over a communication channel.

- MAC Throughput: the average quantity of data frames that can be delivered successfully over a communication channel at the MAC sub-layer.

- Packet end-to-end delay: the average time taken by a data packet to arrive at the coordinator. Measurement of the packet delay starts from the time when data frames enter the MAC sub-layer.

\section{Packet Delivery Ratio and Collision Rate}

In this chapter, the reliability of WBAN upon using CLFB is examined based on PDR and Number of Collisions. Table 3.3 compares PDR achieved by CLFB with four competing algorithms, i.e. IEEE 802.15.4, ACS [163], $\mathrm{D}^{2} \mathrm{MAC}$ [107] and another competing algorithm [31], which to facilitate our discussion, will be called "NB-Step". As it is shown in Table 3.3, when there are two and three nodes in the network, PDR is nearly $100 \%$ and there are no statistically significant differences among the different algorithms. While the number of nodes becomes larger, PDR starts to deviate. For example, when there are nine nodes, PDR for the five algorithms will be 0.63 (IEEE 802.15.4), 0.63 (ACS), 0.82 (D2MAC), 0.81 (NB-step) and 0.77 (CLFB), respectively. With the help of the t-test, CLFB is shown to be statistically more reliable (i.e. higher PDR) than IEEE 802.15.4 and ACS.

Although, CLFB can significantly improve the communication reliability in comparison to IEEE 802.15.4 and ACS, the results show $\mathrm{D}^{2} \mathrm{MAC}$ and NBstep can manage to achieve higher PDR than CLFB. This happens because they impose significantly longer backoffs on CSMA /CA. As a result the packet delay increases substantially. Different from these two competing algorithms, CLFB can keep the delay close to the level obtained in IEEE 802.15.4. In the meantime, it can still achieve higher PDR. 
After investigating PDR, the collision rate is also considered. Generally, by increasing the number of nodes, the PDR will decrease in all algorithms. This is because of the increasing number of collisions on the network, as shown in Table 3.4. Statistically, t-test shows that CLFB can perform significantly better than IEEE 802.15.4 and ACS when more than two nodes are involved in the simulation. On the other hand when only two nodes are simulated, no collisions can be observed. Although the results show that $\mathrm{D}^{2} \mathrm{MAC}$ and NB-step achieved lower collisions, they sacrifice the delay hugely. We found that the real advantage of CLFB over D ${ }^{2}$ MAC and NBstep is due to its capability of decreasing collisions without noticeably increasing communication the delay of IEEE 802.15.4.

\section{MAC Throughput}

Table 3.5 presents the performance of the network in terms of the MAC throughput. T-test shows that CLFB statistically outperforms IEEE 802.15.4 and ACS when there are more than two sensor nodes in the network.

Meanwhile, when the network has nine nodes, statistically CLFB performs the same as $D^{2} \mathrm{MAC}$ and NB-step. Nevertheless, the simulation results show the throughputs of $\mathrm{D}^{2} \mathrm{MAC}$ and NB-step in other cases outperform CLFB. That happens because they can avoid more collisions by significantly increasing the backoff delay. However, long backoff delays may not be desirable for delay sensitive applications.

\section{Packet end-to-end delay}

As we mentioned earlier, the simulation results illustrated in Table 3.6 show that $\mathrm{D}^{2} \mathrm{MAC}$ and NB-step introduced significantly higher delays to the network than other algorithms. For example, our t-test analysis indicates that CLFB can manage to achieve markedly less delay than $\mathrm{D}^{2} \mathrm{MAC}$ and NBstep. In fact, the delay achieved by CLFB is statistically indistinguishable from that of IEEE 802.15.4 and ACS. As we further highlighted in Table 3.6, 


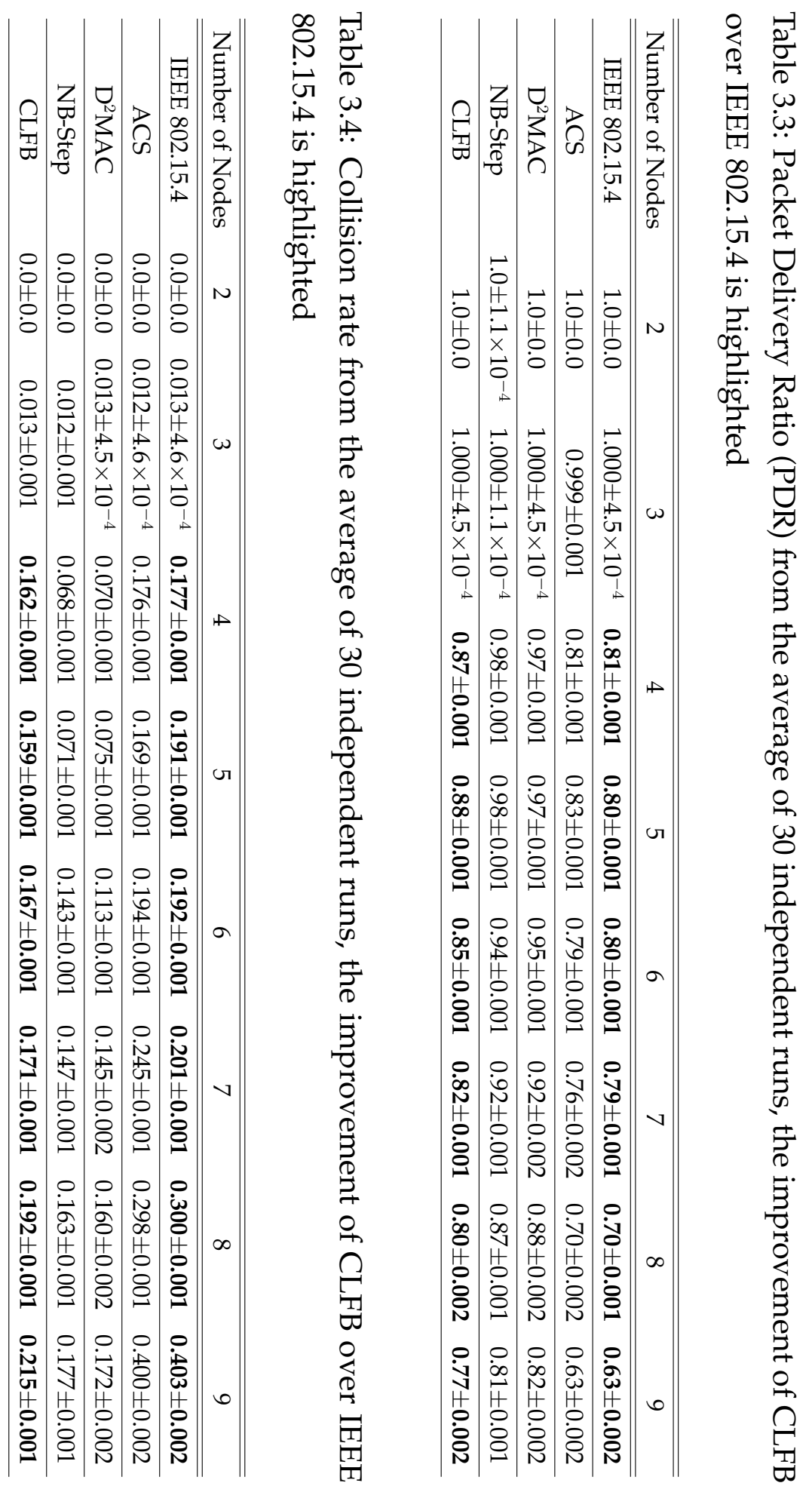


in some simulations, CLFB can even achieve slightly less delay than IEEE 802.15.4.

Based on the observations above, we can claim that the main strength of CLFB lies in the fact that it can statistically dominate the performance of IEEE 802.15.4 in terms of both the reliability (e.g. PDR) and performance (e.g. delay). Particularly, among all the competing algorithms, CLFB is the only algorithm that can outperform IEEE 802.15.4 in every metric. This leads us to believe that CLFB can achieve the best utilisation of the limited communication bandwidth so as to realize a good balance among all performance metrics.

\subsection{Chapter Summary}

In this chapter, we proposed the Cross-Layer Fuzzy logic based Backoff system (CLFB) to improve network reliability, i.e. Packet Delivery Ratio (PDR) and collision rate. We also aimed to improve the throughput in WBANs without increasing packet delay to a great extent. CLFB was designed by us to produce the Backoff Exponent (BE) by considering both the channel condition and the application data rate. This design brings higher levels of adaptability to WBANs. In addition, we also presented a manual approach to fine-tune the fuzzy membership functions in CLFB in order to enhance its effectiveness. By integrating our CLFB into the IEEE 802.15.4 MAC sub-layer, we successfully enhanced the competence of this IEEE standard for various WBAN-based applications. Moreover, this integration does not significantly change the underlying structure of the IEEE 802.15.4. Thus, backward compatibility is ensured. The results clearly showed that our CLFB achieved noticeable improvement in network reliability and performance. In the meantime, the packet delay was still maintained at a reasonably low level, consistent with the original standard.

One possible limitation of the current solution is its reliance on manual design for FLCs used in CLFB. Hence, in Chapter 4, we will consider using 


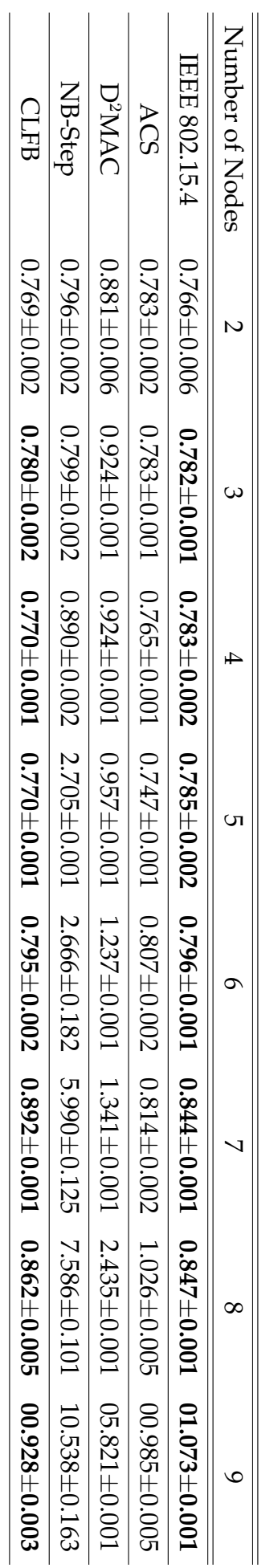

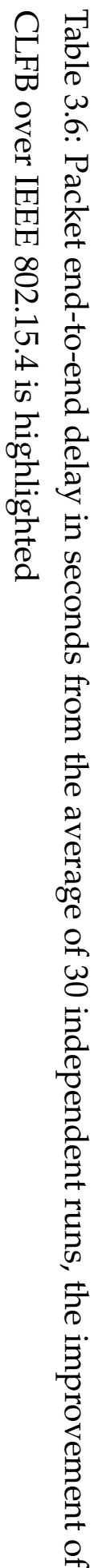

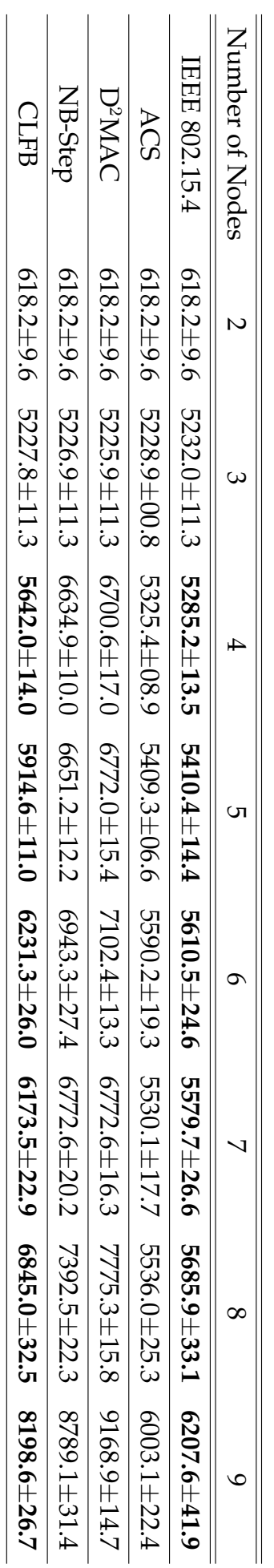

윰

بَ بَ

8

足

옥

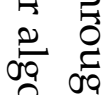

옹

突

क

के

ट.

它

$\begin{array}{cc}09 & 0 \\ 0 & 0 \\ 0 & 0 \\ 0 & 0\end{array}$

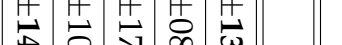

옹

官

0
0
0
0
0
0
0
0

웅

फ

5
2
0
0
0
0
0
0
2
2
5
5

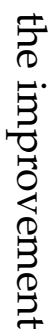


automatic design based on soft computing techniques such as Particle Swarm Optimisation (PSO) and Deferential Evolution (DE) algorithms, to effectively find suitable Membership Function (MFs) in FLCs used in CLFB.

Another possible limitation of the current solution is its focus primarily on re-engineering the standard slotted Carrier Sense Multiple Access with Collision Avoidance (CSMA/CA) in IEEE 802.15.4. Further performance and reliability improvement can potentially be achieved by combining CSMA/CA and Guarantee Time Slots (GTS). Our research shows that, by improving CSMA/CA alone, higher levels of network reliability and performance can be achieved. This technological improvement is complementary with use of GTS. The potential use of GTS will be considered in Chapter 5. 


\section{Chapter 4}

\section{Automatic Design of Fuzzy Logic Controller in Wireless Body Area Networks}

\subsection{Introduction}

In the previous chapter, we addressed the limitations of commonly used IEEE 802.15.4 in WBANs by proposing Cross-Layer Fuzzy Logic based Backoff (CLFB) to improve network reliability and performance. We improved network performance by relying primarily on manual approaches for designing Fuzzy Logic Controllers (FLCs) used in CLFB, even though, they are laborious and error-prone [84]. This issue has not received substantial attention in the literature. Hence, in this chapter, we will study the usefulness of automatic methods for systematic design of FLCs in WBANs.

Amongst various automatic techniques used to design FLCs, Evolutionary Computation (EC) techniques are very effective at handling noisy and multi-modal problems [45]. Due to this reason, EC-based automatic design of FLCs will be evaluated comprehensively in this research. In particular, in order to demonstrate that our approach can effectively work with commonly used EC techniques, we will examine the usefulness of two well- 
known EC techniques, i.e. Particle Swarm Optimization (PSO) [17, 65, 102, 52] and Differential Evolutionary (DE) [59, 27, 124, 36]. These two algorithms have been chosen because of our familiarity and their proven effectiveness $[43,80,157,102,59,52,113]$. However, we do not rule out the possibility of using other $\mathrm{EC}$ techniques.

In order to evolve useful FLCs by using EC techniques, four important technical issues must be addressed. The first issue focuses on the coding scheme which turns an arbitrary design of FLC into a candidate solution that can be evolved by EC techniques. In order to produce an effective design of FLCs, we jointly investigate the efficacy of three different coding schemes with varied levels of restrictions. Our research shows that less restricted coding schemes may help to improve the performance of WBANs. However, a good balance between performance and interpretability is essential in practice.

The second issue is to design a meaningful fitness function to guide the evolutionary process effectively. Our primary goal is to improve the reliability (i.e. PDR) and performance (i.e. throughput and packet delay) of WBANs. In this research, throughput is implicitly considered as it is indirectly improved by enhancing PDR. However, increasing PDR may lead to an increase in packet delay. Consequently, PDR is constrained by packet delay. It is intuitive to pursue PDR as the main objective and packet delay as a constraint. In our approach, whenever the packet delay of candidate FLCs is as good as IEEE 802.15.4, the fitness function will focus mainly on improving PDR. On the other hand, if there is a deterioration in packet delay in comparison to IEEE 802.15.4, the fitness function will be penalised to achieve a good balance between PDR and packet delay. In our experiments, we successfully show the usefulness and effectiveness of our fitness function for EC-based FLCs design.

The third issue to be studied in this chapter concentrates on different design targets in the context of WBANs. This research defines two alternative design targets. The first target is designing optimum FLCs to provide 
reliability and performance for a specific network configuration in WBAN. The second target is aimed at designing desirable FLCs for more general WBAN settings. To fulfil these design targets, we propose two methods to evaluate candidate FLCs. The first method evaluates candidate FLCs based in a single predefined network setting and is useful for the first target. The second method evaluates candidate FLCs through a combination of multiple varied network settings in an attempt to support the second target. Our experiments show the FLCs evolved for the first target can only perform well on a specific network setting. Any changes to this setting will have a negative impact on the effectiveness of the evolved FLCs. Moreover, we experimentally demonstrate that, although the effectiveness of designing FLCs by using the first evaluation method can outperform the second method, FLCs evolved through the second method can provide desirable reliability and performance for a wider range of WBAN settings. The second method hence brings the ability to add or remove patients sensor nodes and improves the overall applicability of our approach.

The fourth issue considers the efficiency of the EC-based design process. Since evaluating the fitness of any candidate FLC design is time-consuming (each run of PSO or DE takes 119 hours with our High-Performance Computing (HPC) facilities), the efficiency of the design process must be improved. We address this issue by utilising surrogate models to compensate for computation costs. In order to improve the accuracy, the surrogate model will be re-trained through online learning of each generation of fitness evaluation. After examining the usefulness of surrogate-assisted ECs, we found that, although the surrogate model slightly impedes effectiveness, it is still useful because the efficiency of the design process is significantly improved.

\subsubsection{Chapter Goals}

Soft computing techniques including fuzzy logic have been successfully applied to Wireless Body Area Networks (WBANs). However, most of the 
existing research works rely on manual design of the FLC. To address this issue, this chapter aims to propose an evolutionary approach to automate the design of FLCs for cross-layer medium access control in WBANs. The following are the chapter goals briefly:

1. Investigating the effectiveness of coding schemes to encode the candidate solutions. The coding scheme turns an arbitrary design of FLC into a candidate solution that can be evolved by ECs. In the literature, most of the research works only have studied domain-specific coding schemes. Researchers have also studied some general coding schemes. However, it is not clear which coding scheme is particularly useful for our design problem. Consequently, we study three alternative coding schemes with varied levels of restriction.

2. Designing a meaningful fitness function for leading the evolutionary design process to achieve a desirable balance between reliability and performance in WBANs. The primary design goal is improving the reliability of WBANs, while the performance of the network is remaining competitive with respect to IEEE 802.15.4.

3. Defining design targets based on different WBAN's scenarios to evaluate the fitness and improve the practicality of our approach.

4. Improving the efficiency and practicality of the design approach via surrogate models.

5. Investigating the usefulness of commonly used EC techniques to design FLCs through our approach.

6. Demonstrating the practical usefulness of our EC-based FLC design approach in WBANs by experimentally comparing it with several existing algorithms such as CSMA/CA in IEEE 802.15.4, Additional Carrier Sensing (ACS) [163], Dynamic Delayed Medium Access Control $\left(\mathrm{D}^{2} \mathrm{MAC}\right)$ [107] and a recent algorithm proposed by Chen et al. [31]. 
For the sake of simplicity, in this chapter, "IEEE 802.15.4" refers to the standard CSMA/CA algorithm.

\subsubsection{Chapter Organisation}

The remainder of this chapter is organised as follows. Section 4.2 proposes the EA-based FLCs design in WBANs. Section 4.3 reports and analyses the simulation results. Finally, in Section 4.4 the summary will be presented.

\subsection{Evolutionary Design of FLCs in WBANs}

In this section, we propose a general method to automatically design FLCs in CLFB in order to obtain a desirable balance between reliability and performance in WBANs. As reported extensively in the literature [159, 45, $59,11,47]$, EC techniques have been applied to automate the design of various aspects of FLCs. As shown in Figure 4.1, the focus of this chapter is primarily on designing the control parameters of all MFs in a FLC.

Particularly, in the context of WBANs, we identify four important technical issues for EC-based design of FLCs: (1) coding scheme, (2) fitness function, (3) evaluation method and (4) efficiency. These issues will be addressed respectively in the following subsections. To facilitate our study, in this chapter, PSO and DE have been selected to drive the automatic design process due to their proven effectiveness [43, 80, 157, 102, 59, 52]. It is important to note that our aim is to demonstrate that this approach can work well with many commonly used EC techniques. We choose PSO and DE in particular because of our familiarity, but do not rule out the possibility of using other EC techniques.

\subsubsection{Coding Scheme}

Most EC techniques operate primarily on a population of candidate solutions. Each candidate solution represents a separate FLC design and 


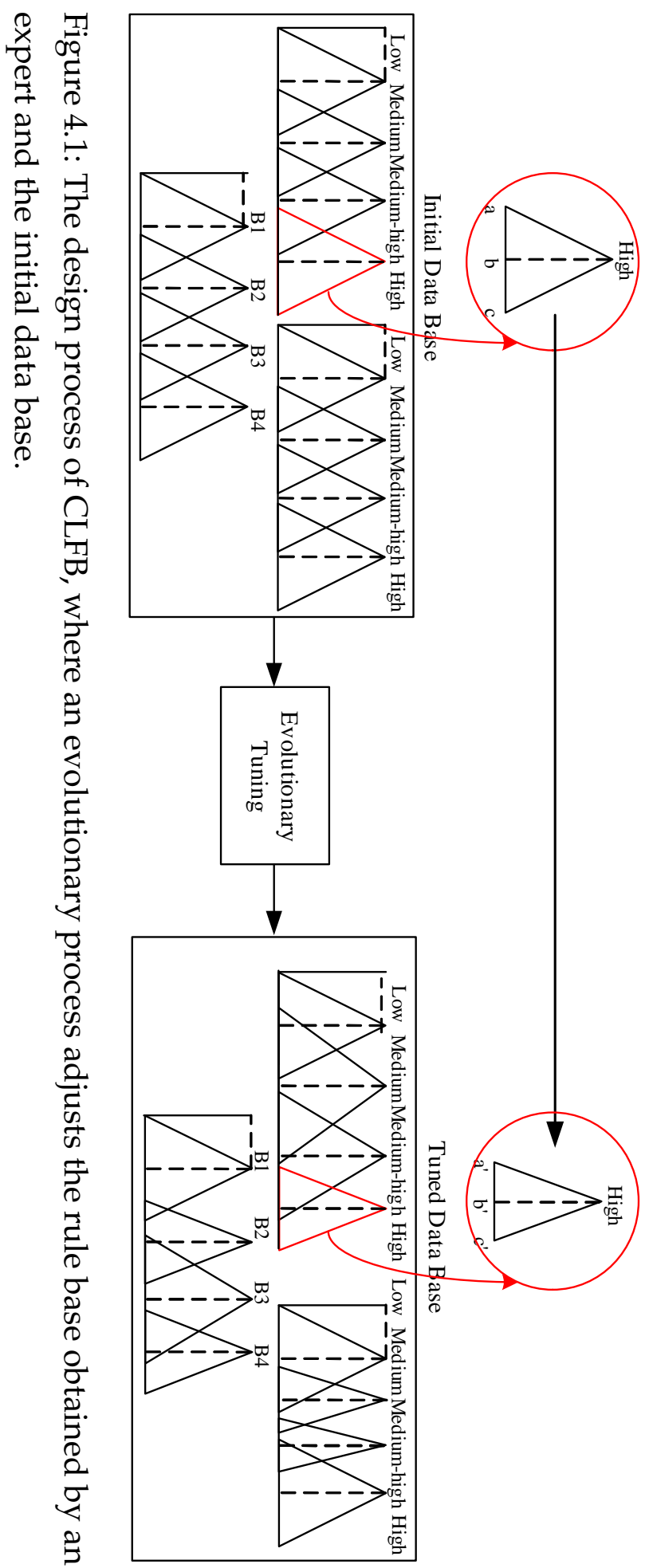


follows a specific coding scheme which encodes all control parameters for each MF through a vector of real numbers. In this vector, every three consecutive dimensions form an individual group of control parameters. For example, as depicted in Figure 4.2, the first group of parameters, which control the first MF, have been highlighted in red. Due to the use of triangular MFs, the first control parameter in any group $(a, b, c)$ must always be less than the middle-control parameter $(a<b)$, which subsequently must always be less than the third control parameter $(b<c)$.

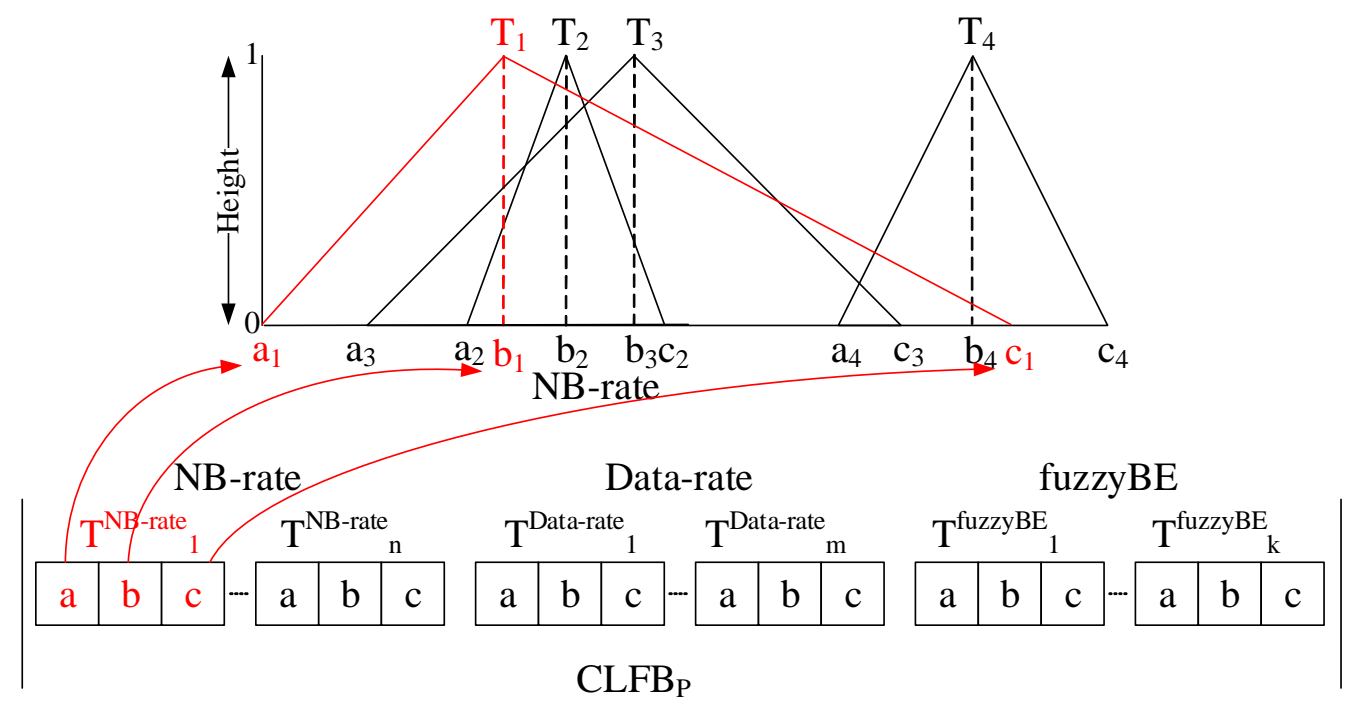

Figure 4.2: Unrestricted coding scheme (URCS) in which $T$ is the linguistic of each membership function.

As illustrated in Figure 4.2, the dimension of every candidate solution is directly related to the number of MFs. Because our FLC in Figure 3.3 has two input variables and one output variable, every variable is modelled by four MFs, and each MF includes three control parameters, the dimension of each candidate solution is $3 \times 4 \times 3=36$.

The coding scheme explained above gives a flexible method to encode candidate solutions. For convenience of discussion, we will call this coding scheme the UnRestricted Coding Scheme (URCS). As depicted in Figure 4.2, 
the domain of any MF in URCS can be completely covered by the domains of other MFs (e.g. the domain of MFs $T_{2}$ and $T_{3}$ is completely enclosed by the domain of MF $T_{1}$ ). Consequently, MFs cannot be easily distinguished for good interpretability. Hence, it is difficult to associate semantic (domainspecific) meanings to these MFs [178]. In order to design more interpretable FLCs, we will introduce two other coding schemes with different levels of restrictions. They are the Strictly Restricted Coding Scheme (SRCS) and the Moderately Restricted Coding Scheme (MRCS). In comparison with URCS, overlapping between different MFs is more strictly controlled in SRCS and MRCS.

SRCS imposes a stringent restriction on each MF. Particularly, as illustrated in Figure 4.3, the middle control parameter of each MF can be covered only by itself and not by any other MFs. Thus any MF can only partially overlap with its neighbouring MFs. In other words, any crisp input to our FLC in CLFB cannot be covered by more than two MFs simultaneously.

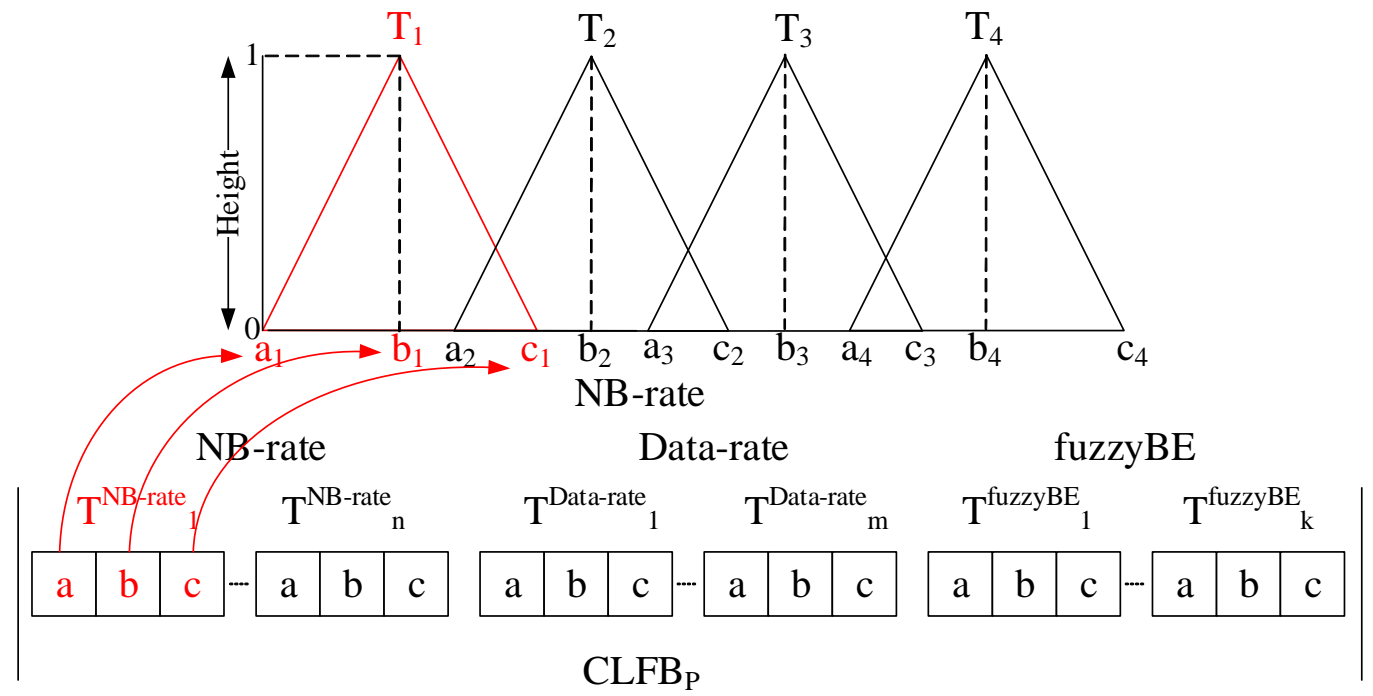

Figure 4.3: Strictly restricted coding scheme (SRCS) in which $T$ is the linguistic of each membership function.

Different from SRCS, MRCS presents a more flexible restriction by util- 
ising special Overlapping Control Factors (OCFs) in the candidate solution as shown in Figure 4.4. In particular, each membership function is associated with one OCF. OCF is used to decide (1) whether the right tail of the neighbouring MF to the left can cover its middle-control parameter (i.e. $\mathrm{OCF}<0.33$ ) or (2) whether the left tail of the neighbouring MF to the right can cover its middle-control parameter (i.e. $033 \leqslant \mathrm{OCF} \leqslant 0.66$ ). On the other hand, if $\mathrm{OCF}>0.66$, no neighbouring MFs will cover the middle-control parameter. Obviously, when MRCS is used, 12 new dimensions must be added to each candidate solution (due to the fact that we have $3 \times 4=12 \mathrm{MFs}$ in the FLC). Consequently, the total dimension of each candidate solution becomes $36+12=48$.

In the literature, most of the research works have only studied domainspecific coding schemes. Some researchers have also studied general coding schemes [23]. However, it is not clear which coding scheme is particularly useful for our design problem. Therefore, in this research, in order to discover an effective coding scheme, the three coding schemes with varied levels of restrictions will jointly be studied in the context of WBANs (refer to Section 4.3.)

\subsubsection{Fitness Function}

Fitness function is an essential part of our design approach. Through properly designed fitness functions, we hope to obtain a desirable balance between reliability and performance in WBANs. In order to measure reliability, PDR (widely used in the literature [119]) has been utilised in this chapter. PDR is calculated as the ratio of packets that are successfully delivered to the coordinator over the number of packets that have been sent out by all sensor nodes in a WBAN as below:

$$
P D R=\frac{\text { Number of Packets Received by Coordinator }}{\text { Total Number of Packets Sent by all Sensor Nodes }}
$$

Obviously, when PDR is high, critical data will experience fewer collisions during transmission in WBANs. To achieve this objective, we define 


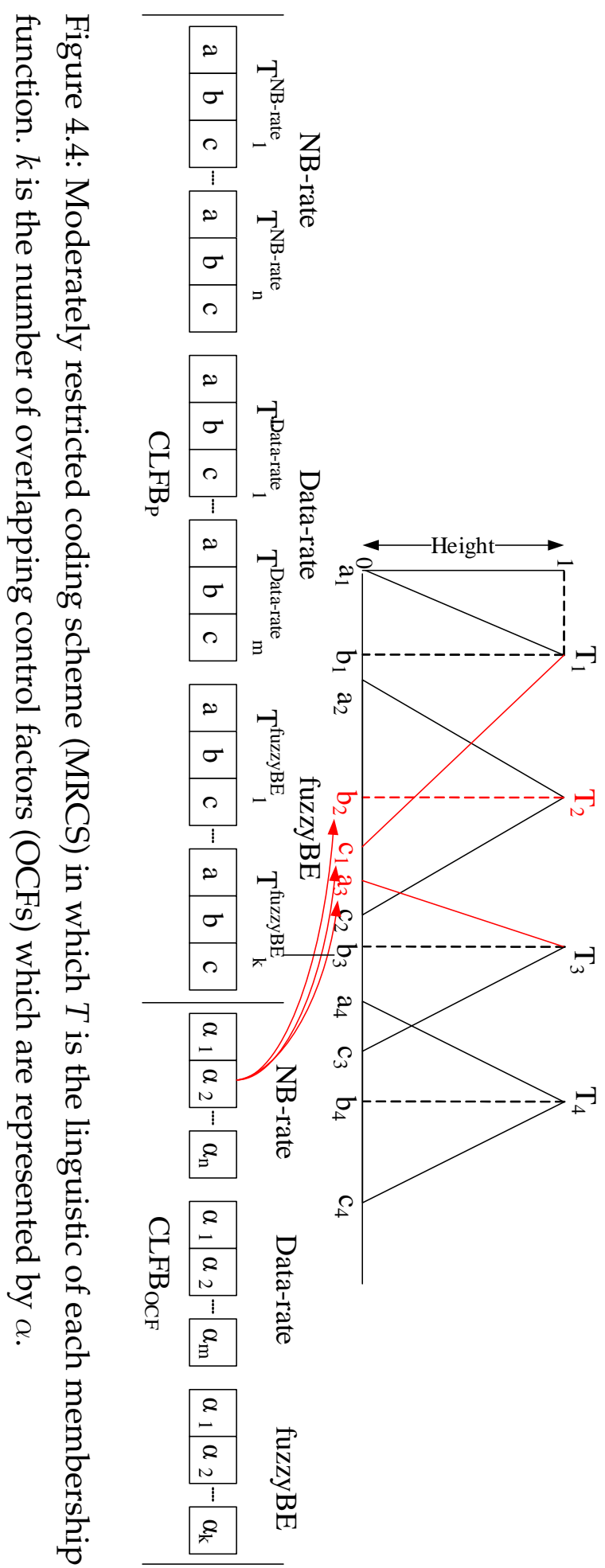


$\triangle P D R$ as follows:

$$
\triangle P D R=P D R_{C L F B}-P D R_{s t d}
$$

where $\mathrm{PDR}_{C L F B}$ and $\mathrm{PDR}_{s t d}$ refer to PDRs obtained by utilising CLFB and IEEE 802.15.4 respectively. Whenever $\triangle P D R>0$, the reliability achieved by CLFB is considered better than that of IEEE 802.15.4.

Besides reliability, it is also highly desirable to improve the performance of WBANs. Performance can be measured by two metrics, which are packet delay and network throughput. Since enhancement in throughput is realised by improving PDR, throughput is implicitly considered in this research. Consequently, the main focus of this chapter is on packet delay. $\Delta$ Delay is subsequently defined as follows:

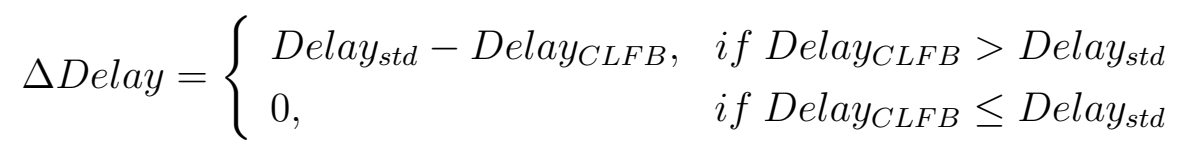

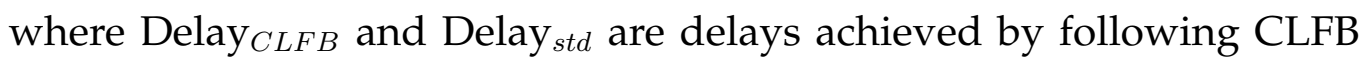
and IEEE 802.15.4 respectively. Since the packet delay in IEEE 802.15.4 is generally considered acceptable for many WBANs applications [20], instead of maximising $\triangle$ Delay, we want CLFB to achieve the same level of delay as IEEE 802.15.4. Therefore, when Delay D $L F B>$ Delaystd $_{\text {st }}$ (4.3), the delay achieved by FLC in CLFB is considered as less desirable and $\triangle$ Delay becomes negative. On the other hand, if Delay ${ }_{C L F B} \leq$ Delay $_{\text {std, }}$, this time CLFB has performed well enough and $\Delta$ Delay will be fixed at zero. Due to (4.3), $\Delta$ Delay behaves essentially as a constraint. When the delay becomes reasonably short, the main focus of our design process will be primarily on maximising PDR.

As explained above, $\triangle P D R$ in (4.2) is the main objective to be optimised, and $\Delta$ Delay in (4.3) serves as a constraint for the purpose of keeping the delay achieved by FLC in CLFB as competitive as IEEE 802.15.4. Consequently, the fitness function is defined as follows:

$$
F=\Delta P D R+\lambda \times \Delta \text { Delay }
$$


where $\lambda$ is a coefficient. As a simple example, when $P D R_{C L F B}=0.50$ and $P D R_{s t d}=0.75$ then $\triangle P D R=0.50-0.75=-0.25$. In this case, if $\Delta$ Delay $=0$, the fitness function $F$ in (4.4) becomes -0.25 . This means that the packet delay achieved by the candidate FLC is as good as IEEE 802.15.4 and $\triangle P D R$ can be improved without any penalty. This chapter aims at finding a proper coefficient to obtain a desirable trade-off between PDR and delay. The particular effect of $\lambda$ will be further studied in Section 4.3.

\subsubsection{Fitness Evaluation Method}

In this chapter, two methods are studied to evaluate the fitness of each candidate solution guided by two different design targets. The first design target $\left(\mathrm{T}_{1}\right)$ is to design FLCs that function effectively on a specific network setting. For example, in a healthcare application for monitoring an elderly individual who has lately been sent home following recovery from a cardiac surgical procedure, a pre-defined WBAN setting with a three-lead ECG, blood pressure (BP) and heart rate (HR) sensors is required [2]. Therefore, our aim is to improve reliability and performance in this particular setting. The second target $\left(\mathrm{T}_{2}\right)$ is to design FLCs that are able to work consistently well across a wide range of network settings. Target $T_{2}$ aims to improve the general applicability of FLCs. To distinguish design targets, every result upon using our CLFB based on targets $T_{1}$ and $T_{2}$ will be refer to as $C L F B_{T_{1}}$ and $\mathrm{CLFB}_{T_{2}}$ respectively.

In association with the two design targets, we consider two different evaluation methods. The first method evaluates the candidate solution based on a single pre-defined WBAN setting. The second evaluation method utilises multiple pre-determined WBAN settings to jointly evaluate the same candidate solution. Therefore, in the second approach, the averaged $\triangle P D R$ and $\triangle$ Delay over multiple network settings is utilised to determine the fitness of the candidate according to (4.2), (4.3) and (4.4). In both evaluation methods, every network setting is tested for five independent runs. For detailed information, please refer to Subsection 4.3.5. 
In this research we are not employing any overfitting control techniques while designing FLCs. However, we have experimentally demonstrated the effectiveness of designing FLCs through EC-based approach to improve the reliability and performance of varied WBAN's settings. In the future work, we will take advantage of using overfitting control techniques [35].

\subsubsection{Surrogate Assisted ECs for Designing FLCs}

In this subsection, we investigate the usefulness of surrogate models to improve the efficiency of EC-based design of FLCs in WBANs. EC techniques usually require a large number of fitness function evaluations before converging to a good FLC design [74]. Unfortunately, fitness evaluations may often become costly. Such situations typically occur when EC techniques are employed to solve expensive problems, such as the time-consuming simulation of WBANs introduced in Subsection 4.2.2 and 4.2.3. To address this efficiency issue, this chapter utilises a surrogate model to approximate the fitness of a certain proportion of candidate FLC designs evolved by EC techniques.

Various methods have been introduced for constructing surrogate models $[75,74]$. In particular, the widely used methods in design engineering include Kriging models, feed-forward Multi-Layer Perceptrons (MLPs) and Radial Basis Function Networks (RBFNs) [76]. Comparison has been made by some researchers to examine the effectiveness of different surrogate models $[22,73,74]$. However, because the performance might be problem dependent, it is difficult to choose the best model for any specific design tasks [74]. Since the focus of this research is only on investigating the usefulness of surrogate models for improving the efficiency of the design process, we have identified three commonly used criteria while building our surrogate model: simplicity, accuracy, and direct support for online learning.

Simplicity and accuracy are widely addressed in the literature [74]. Obviously, simple models, which are easy to implement and use, are desirable. 
However, a simple model (e.g. low order polynomial models) might perform poorly in our high-dimensional design method. Therefore, in order to make a reasonably good balance between accuracy and simplicity, we have selected MLPs to approximate high-dimensional fitness function. From our preliminary study, we found that MLP can achieve reasonable accuracy with limited sample data.

MLP also supports the third design criterion since it can be learned online during the design process. This feature helps the model accuracy, so that it can be more reliably used to guide EC-based design [74]. However, we are not excluding the possibility of using other surrogate models. Figure 4.5 shows the MLP with one hidden layer that approximates the fitness for each candidate FLC design. The input variables of our MLP are the control parameters of MFs determined by the coding schemes in Subsection 4.2.1.

As shown further in Figure 4.6, the whole population of the first generation is evaluated based on the real fitness function. A dataset of sample fitness values is created as a result. It is subsequently used to train the MLP model. Afterwards, the MLP is utilised to replace the real fitness function for evaluating a certain proportion of candidate solutions in the population. Meanwhile, in order to improve its accuracy, our MLP is re-trained based on the newly added fitness samples obtained from evaluating the remaining candidate solutions by using the real fitness function.

Following the idea described above, during every generation of the EC techniques, a number of controlled individuals $(\eta)$ in the population (NP in total) are chosen to be re-evaluated through network simulations. There are two typical strategies to select individuals for re-evaluation [76]. The first strategy is to select controlled individuals completely randomly. The second strategy is to select the best $\eta$ individuals (with the highest fitness values) as the controlled individuals. Intuitively, good individuals are more important since they tend to have stronger influence on future generations $[76,75]$. Therefore, the second strategy is utilised in our experiments. Also, the $\eta$ selected controlled individuals and their "real" fitness values will be 


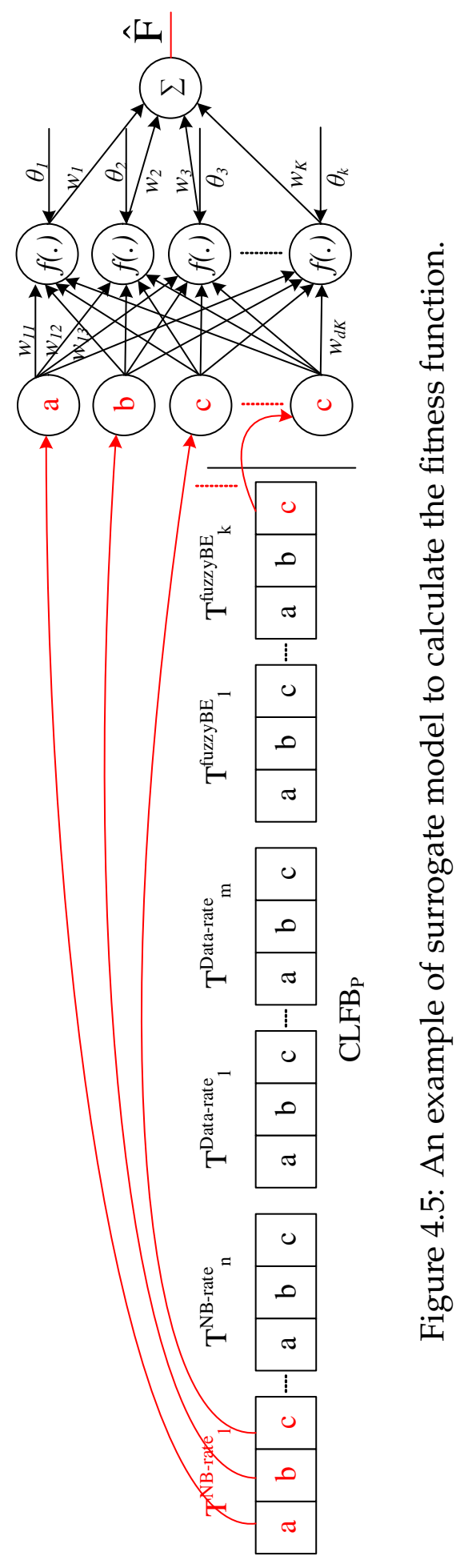




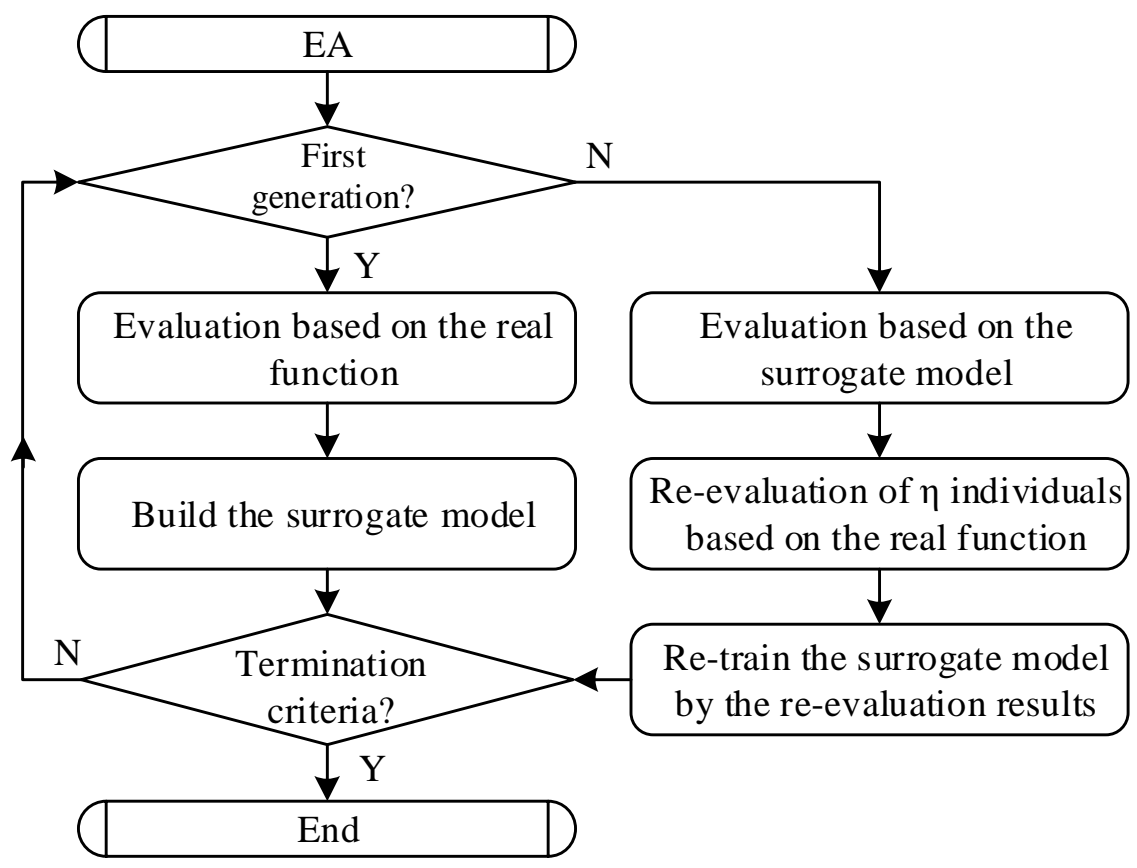

Figure 4.6: An example of surrogate model to calculate the fitness function.

exploited to re-train our MLP incrementally.

\subsection{Simulation Implementation and Results}

In this section, we describe the simulation settings for WBANs in Subsection 4.3.1, settings of the EC techniques parameters in Subsection 4.3.2, and the evaluation metrics in Subsection 4.3.3. Afterwards, the simulation results will be reported in Subsections 4.3.4 and 4.3.5. Subsection 4.3.4 presents the results of designing FLCs to achieve target $T_{1}$, i.e. FLCs for specific network settings. Subsection 4.3.5 analyses the simulation results obtained from designing FLCs with target $T_{2}$ in mind, i.e. FLCs for multiple network settings.

In Subsection 4.3.4, firstly, in order to discover proper coefficients for our fitness function in (4.4), we study ten different coefficients. Afterwards, to 
show that our approach can work well with commonly used EC techniques, we present experiments on two well-known EC techniques, i.e. PSO and DE. Then we particularly investigate the effectiveness of three coding schemes. To improve the efficiency of the design process further, the usefulness of surrogate models will be investigated next. Finally, through comprehensive simulation studies, we conclude that our EC-based approach for FLC design can outperform many competing algorithms in the literature, including IEEE 802.15.4, ACS, and $\mathrm{D}^{2} \mathrm{MAC}$, as well as a recently proposed algorithm [31] termed "NB-Step".

In Subsection 4.3.5, firstly, we define multiple scenarios for automatic design of FLCs for target $T_{2}$. Similar to Subsection 4.3.4, three coding schemes will be compared. Then the effectiveness and efficiency of using surrogate models will be investigated. Finally, the simulation studies on several testing scenarios will be conducted to demonstrate that our EC-based approach for FLC design can outperform many competing algorithms across many different network configurations.

\subsubsection{Simulation Environment for WBANs}

In order to simulate WBAN, we have utilised OMNeT++ network simulator version 4.4.1 [5]. Following typical settings in the literature [119], the maximum data rate is $250 \mathrm{~Kb} / \mathrm{s}$ with frequency of $2.4 \mathrm{GHz}$. In our simulation, all sensor nodes are randomly deployed within a $2 \times 2 \mathrm{~m}^{2}$ space with a single WBAN coordinator using the star topology. The network simulation time for our experiments is 600 seconds (longer simulation time does not appear to alter our major findings). We have used the log-normal shadowing model as the channel model. In comparison with the traditional Rayleigh and Ricean distributions, the log-normal shadowing model reproduces the small-scale fading in WBANs more accurately [147]. 


\subsubsection{Parameter Settings for Evolutionary Algorithms}

The aim of this research is to show that our approach can effectively work with different EC techniques. Therefore, two well-known EC techniques, i.e. PSO and DE with standard operators, are utilised to evolve effective FLC designs. We have no intention to introduce any changes to PSO or DE which are beyond the scope of this chapter. For both PSO and DE, the population size is set to 50 . The maximum number of generations is set to 100. Under these settings, both PSO and DE are close to convergence after 100 generations, as evidenced in Figure 4.7.

Based on the common settings in the literature $[167,134]$, the fully connected topology is used for PSO. Meanwhile, $\omega=0.7298$ and $c_{1}=c_{2}=1.49618$ $[167,134]$. For DE, we choose the standard method DE/rand/1/bin, where Crossover Rate (Cr) and scaling factor (F) are set to 0.8 and 0.5 respectively [29].

To obtain reliable results, PSO and DE under the same settings are repeated 30 times with different starting seeds. In addition, the Analysis of Variance (ANOVA) test is performed to determine whether statistically significant differences in performance and reliability can be observed. Tukey's post-hoc analysis and t-test analysis are also utilised to realise the main source of difference.

\subsubsection{Evaluation Metrics}

To quantitatively compare the reliability and performance of WBANs, we employ four well-known metrics as follows:

- Packet Delivery Ratio (PDR): the ratio of the number of sent data packets to the number of delivered data packets as shown in (4.1).

- Collision Rate: the average number of data packet collisions over a communication channel. 


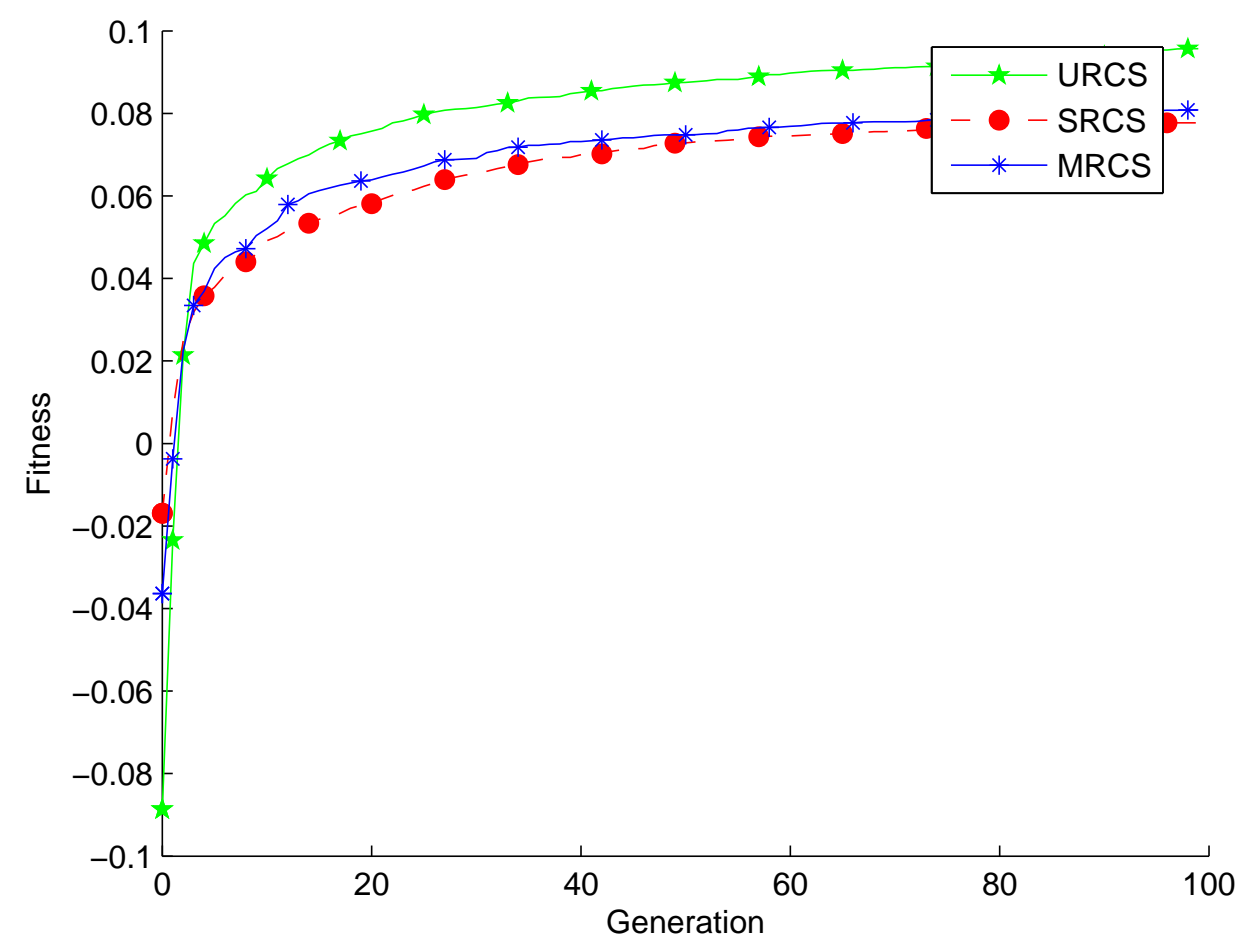

Figure 4.7: The PSO-based process for FLC design will converge after 100 generations. The results for all coding schemes are obtained by averaging over 30 independent tests. 
- MAC Throughput: the average quantity of data frames that can be delivered successfully over a communication channel at the MAC sub-layer.

- Packet end-to-end delay: the average time taken by a data packet to arrive at the coordinator. Measurement of the packet delay starts from the time when data frames enter the MAC sub-layer.

For a fair comparison with competing algorithms, each competing technology is run for 30 times independently on every simulation scenario. Again, the average results in reliability and performance will be used to compare with FLCs designed by our EC-based approach.

\subsubsection{Automatic Design of FLCs for Specific Network Set- tings (Target $\mathbf{T}_{1}$ )}

This subsection introduces a WBAN simulation scenario for target $T_{1}$. Afterwards, the effectiveness of different coding schemes in PSO and DE for FLC designs is statistically investigated. Then, the influence of varied coefficients (i.e. $\lambda$ ) in the fitness function (4.4) will be examined. The efficiency of the design process will further improved through using surrogate models, and finally the reliability and performance of CLFB achieved by FLCs designed in this research are compared with the competing algorithms.

\section{Network Simulation Scenario for Target $T_{1}$}

In this study, we consider a particular application where an elderly individual lives alone. She has lately been sent home following recovery from a procedure. She has a collection of sensors to monitor her health status. A WBAN is responsible for collecting and sharing all sensory information with her doctor via the Internet. This WBAN scenario contains three-lead ECG, EEG, respiratory rate and motion sensors as well as a smartphone 
coordinator. The communication features of these sensor nodes can be found in Table 4.1.

Table 4.1: Communication specifications of Sensor nodes used in the simulation $[2,119]$.

\begin{tabular}{|l|c|c|c|c|}
\hline Sensor Node & ECG & $\begin{array}{l}\text { Respiratory } \\
\text { Rate }\end{array}$ & $\begin{array}{l}\text { Motion } \\
\text { Sensor }\end{array}$ & $\begin{array}{l}\text { Heart } \\
\text { Rate (HR) }\end{array}$ \\
\hline $\begin{array}{l}\text { Traffic } \\
\text { Generation } \\
\text { Distribution }\end{array}$ & Constant & Constant & Poisson & Constant \\
\hline Data Rate & $156.25 \mathrm{Bps}$ & $15 \mathrm{Bps}$ & $64 \mathrm{Bps}$ & $20 \mathrm{Bps}$ \\
\hline \hline Sensor Node & Temperature & EEG & $\begin{array}{l}\text { Blood } \\
\text { Pressure (BP) }\end{array}$ & $\begin{array}{l}\text { Endoscope } \\
\text { Imaging }\end{array}$ \\
\hline $\begin{array}{l}\text { Traffic } \\
\text { Generation } \\
\text { Distribution }\end{array}$ & Poisson & Constant & Constant & Poisson \\
\hline Data Rate & $12.5 \mathrm{Bps}$ & $31.25 \mathrm{Bps}$ & $512 \mathrm{Bps}$ & $1538.46 \mathrm{Bps}$ \\
\hline
\end{tabular}

\section{PSO-based FLCs Design}

Using the WBAN scenario just described, the results from PSO for the three different coding schemes (i.e. URCS, SRCS and MRCS) and different coefficients (i.e. $\lambda$ ) are presented in Table 4.2. From the results, URCS generally outperforms the other two coding schemes, concerning both packet delay and PDR, which is confirmed by ANOVA test and Tukey's post-hoc analysis. For example, when $\lambda=2$ in Table 4.2, we found that URCS provide indistinguishable PDR and significantly less delay compared to MRCS and SRCS (p-values $<0.0001$ ). When $\lambda=2.5$, as another example, URCS statistically shows significantly higher PDR (p-values $<0.0001)$ and indistinguishable delay compared to the other coding schemes. 
Table 4.2: Results obtained by PSO in terms of PDR and packet delay in seconds (target $\left.T_{1}\right)$.

\begin{tabular}{|c|c|c|c|c|}
\hline$\lambda$ & PM & URCS & SRCS & MRCS \\
\hline \multirow{2}{*}{1.0} & PDR & $0.937 \pm 0.004$ & $0.920 \pm 0.002$ & $0.925 \pm 0.002$ \\
\cline { 2 - 5 } & Delay & $0.787 \pm 0.002$ & $0.783 \pm 0.002$ & $0.796 \pm 0.002$ \\
\hline \multirow{2}{*}{1.5} & PDR & $0.893 \pm 0.007$ & $0.904 \pm 0.003$ & $0.882 \pm 0.003$ \\
\cline { 2 - 5 } & Delay & $0.776 \pm 0.006$ & $0.780 \pm 0.002$ & $0.763 \pm 0.002$ \\
\hline \multirow{2}{*}{2.0} & PDR & $\mathbf{0 . 8 6 2} \pm \mathbf{0 . 0 0 6}$ & $\mathbf{0 . 8 7 0} \pm \mathbf{0 . 0 1 1}$ & $\mathbf{0 . 8 6 6} \pm \mathbf{0 . 0 1 1}$ \\
\cline { 2 - 5 } & Delay & $\mathbf{0 . 7 3 8} \pm \mathbf{0 . 0 0 4}$ & $\mathbf{0 . 7 5 0} \pm \mathbf{0 . 0 0 6}$ & $\mathbf{0 . 7 4 8} \pm \mathbf{0 . 0 0 6}$ \\
\hline \multirow{2}{*}{2.5} & PDR & $\mathbf{0 . 8 3 0} \pm \mathbf{0 . 0 0 6}$ & $\mathbf{0 . 8 0 9} \pm \mathbf{0 . 0 0 2}$ & $\mathbf{0 . 8 1 2} \pm \mathbf{0 . 0 0 3}$ \\
\cline { 2 - 5 } & Delay & $\mathbf{0 . 7 2 3} \pm \mathbf{0 . 0 0 2}$ & $\mathbf{0 . 7 2 2} \pm \mathbf{0 . 0 0 1}$ & $\mathbf{0 . 7 2 2} \pm \mathbf{0 . 0 0 1}$ \\
\hline \multirow{2}{*}{3.0} & PDR & $0.832 \pm 0.004$ & $0.802 \pm 0.002$ & $0.801 \pm 0.002$ \\
\cline { 2 - 5 } & Delay & $0.727 \pm 4.7 \times 10^{-4}$ & $0.727 \pm 5.0 \times 10^{-4}$ & $0.727 \pm 0.001$ \\
\hline \multirow{2}{*}{3.5} & PDR & $0.831 \pm 0.004$ & $0.800 \pm 0.002$ & $0.809 \pm 0.002$ \\
\cline { 2 - 5 } & Delay & $0.727 \pm 0.001$ & $0.727 \pm 0.001$ & $0.727 \pm 4.6 \times 10^{-4}$ \\
\hline \multirow{2}{*}{4.0} & PDR & $0.830 \pm 0.003$ & $0.800 \pm 0.002$ & $0.809 \pm 0.002$ \\
\cline { 2 - 5 } & Delay & $0.727 \pm 3.8 \times 10^{-4}$ & $0.727 \pm 0.001$ & $0.727 \pm 0.001$ \\
\hline \multirow{2}{*}{5.0} & PDR & $0.830 \pm 0.002$ & $0.816 \pm 0.001$ & $0.817 \pm 0.001$ \\
\cline { 2 - 5 } & Delay & $0.727 \pm 3.6 \times 10^{-4}$ & $0.727 \pm 3.4 \times 10^{-4}$ & $0.727 \pm 4.9 \times 10^{-4}$ \\
\hline \multirow{2}{*}{10.0} & PDR & $0.823 \pm 0.002$ & $0.814 \pm 0.002$ & $0.817 \pm 0.002$ \\
\cline { 2 - 5 } & Delay & $0.725 \pm 4.1 \times 10^{-4}$ & $0.725 \pm 3.4 \times 10^{-4}$ & $0.725 \pm 0.001$ \\
\hline \multirow{2}{*}{100.0} & PDR & $0.823 \pm 0.003$ & $0.813 \pm 0.005$ & $0.814 \pm 0.001$ \\
\cline { 2 - 5 } & Delay & $0.725 \pm 3.8 \times 10^{-4}$ & $0.725 \pm 0.001$ & $0.725 \pm 0.001$ \\
\hline \multirow{2}{*}{} & & & & \\
\hline
\end{tabular}


Figures 4.8, 4.9 and 4.10 show examples of FLCs designed through URCS, SRCS and MRCS respectively. As depicted in Figure 4.8, the domain of one MF in URCS can possibly be covered completely by the domains of other MFs. For example, the domain of MF for B2 in Figure 4.8c is completely enclosed by the domain of MF for B3. Consequently, the MFs in URCS cannot be easily distinguished for good interpretability. On the other hand, Figures 4.9 and 4.10 show that the MFs in SRCS and MRCS are completely distinguishable.

Another aspect of interpretability of FLCs is the coverage of fuzzy partitioning [9]. This means that the entire domain of a variable should be covered by the MFs generated, and every data point should belong to at least one of the corresponding fuzzy sets. However, the MFs designed through URCS might miss some points as highlighted in Figure 4.8b. On the other hand, the domains of all variables are covered completely by those MFs generated through SRCS and MRCS.

Despite the interpretability issues, the results in Table 4.2 demonstrate that the performance of URCS is better than SRCS and MRCS. Although historically more priority has been given to the performance, the importance of interpretability is also well recognised. Our simulation results show that our coding schemes (i.e. SRCS and MRCS) can provide a desirable trade-off between interpretability and performance in practice.

The above discussion suggests that SRCS and MRCS are more desirable for interpretability reasons. Moreover, the designed rules can also reveal some new information about WBANs. For example, when both SRCS and MRCS are used, the MF for B1 is consistently designed as a narrow triangular at the left end, as shown in Figures 4.9c and 4.10c. In view of the rules $R^{(1)}, R^{(2)}$ and $R^{(3)}$ in Table 3.1, this observation implies that sensor nodes do not need to wait before packet transmission as long as the channel is not busy.

To provide a good balance between reliability and performance in the network, there is a need to determine suitable coefficients for the fitness 

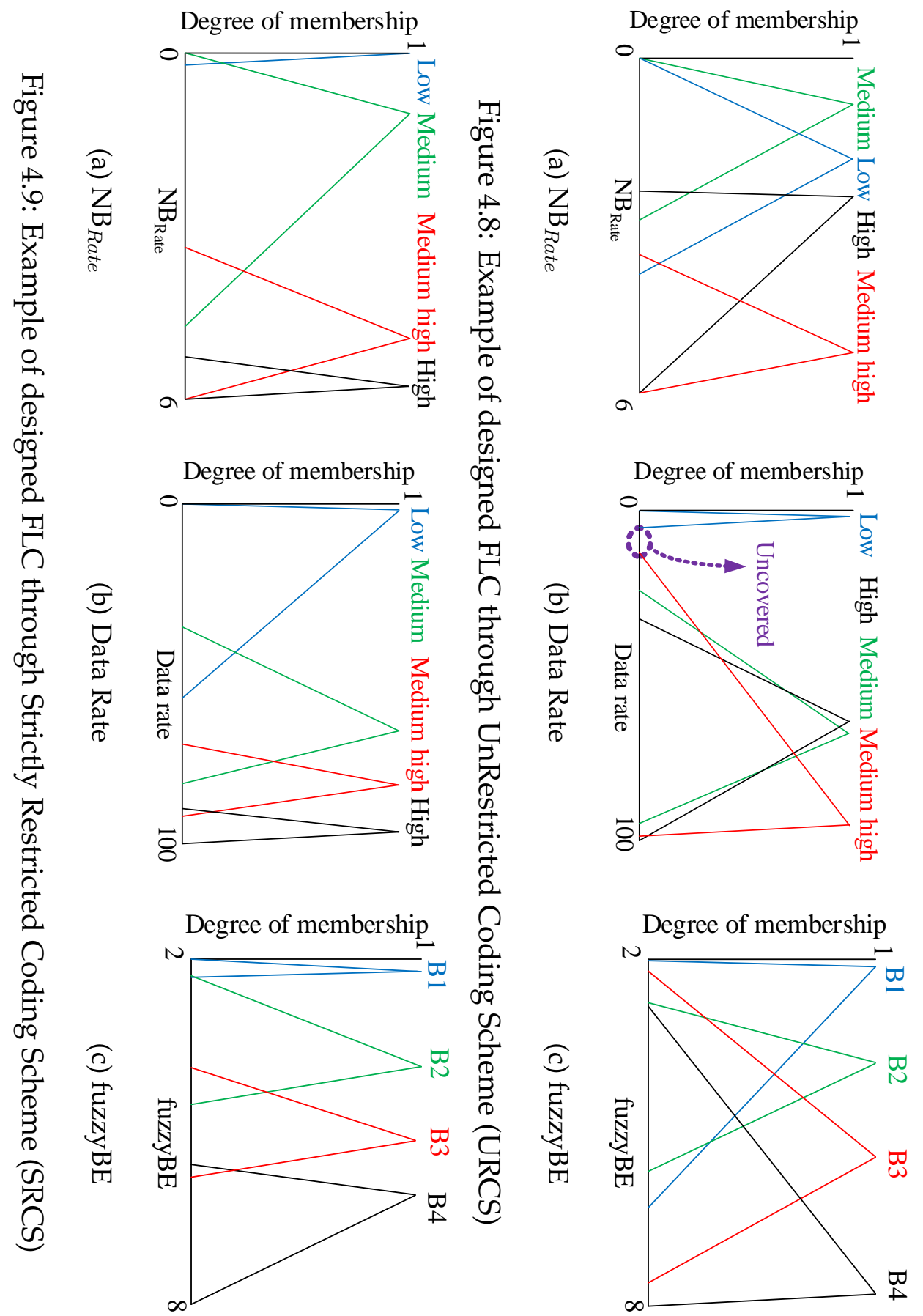


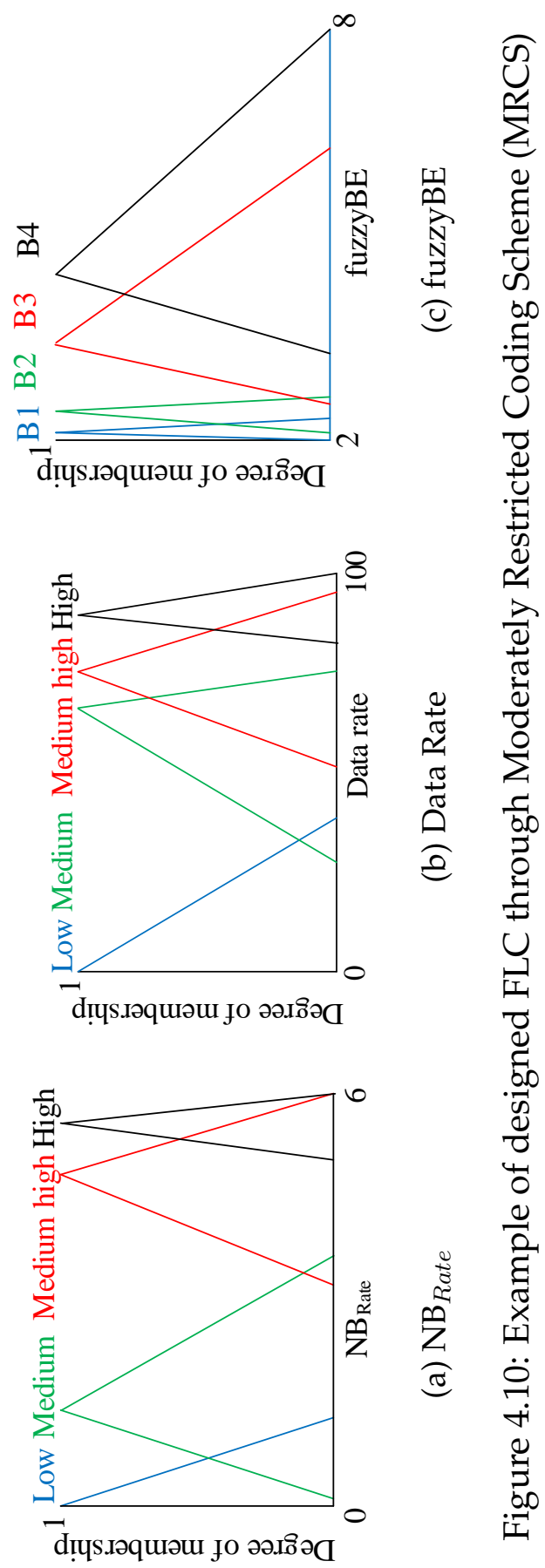


function in (4.4). Therefore, different coefficient for $\Delta$ Delay, i.e. $\lambda$, have been investigated. As evidenced in Table 4.2, we also found that with larger $\lambda$ (e.g. $\lambda>2.5$ ), our EC-based design puts huge attention on keeping the packet delay at a low level (which may not be really necessary). On the other hand, PDR seems to become more influential when $\lambda$ becomes smaller (e.g. $\lambda<2.0$ ). In fact, we have performed a separate one-way ANOVA analysis over all different coefficients for each coding scheme. The results show that, when $\lambda<2.0$, the performance of $\mathrm{CLFB}_{T_{1}}$ will change significantly in delay. Specifically, as shown in Table 4.2, when $\lambda$ changes from 2.0 to 1.5 , delay is increased by 0.038 seconds. On the other hand, when $\lambda>2.0$, the change on delay is always less than 0.015 seconds. Consequently, we will select two coefficients (i.e. $\lambda=2.0$ and $\lambda=2.5$ ) for the rest of our experiments. These two coefficients, which are bolded in Table 4.2, can provide a reasonable balance between PDR and delay. Regardless of which EAs will be used in practice, the design goal remains the same. Hence, only these two coefficients will be considered for the remaining of this subsection.

\section{DE-based FLC Design}

Besides PSO, this chapter also studies the usefulness of DE to determine whether our approach can easily work with different EC techniques. It is obviously not necessary to repeat the experiments above. We hence have utilised only the two specific coefficients identified in the previous paragraph. Moreover, as designing FLCs through EC techniques is highly time-consuming (every run of PSO or DE takes 119 hours with our HPC facilities), this enables us to save considerable computation time. The corresponding results are summarised in Table 4.3.

Although PSO can perform slightly better with URCS than other coding schemes, the one-way ANOVA test and Tukey's post-hoc analysis suggest that, upon using DE, generally no coding scheme is clearly more effective. We notice that FLCs designed through PSO with URCS are statistically more 
effective than those designed through DE, in terms of both PDR and packet delay. Particularly, separate t-tests with respect to each coding scheme and coefficient show significant differences between PSO with URCS and DE with URCS. On the other hand, t-test analysis mostly suggests no significant performance differences between between PSO and DE upon using SRCS and MRCS. There is only one exception: when $\lambda=2.5$. In this case PSO with MRCS outperforms DE. As a result, our statistical analysis suggests that PSO can design FLCs more effectively than DE.

Table 4.3: Results obtained by DE in terms of PDR and packet delay (seconds).

\begin{tabular}{|l|c|c|c|c|}
\hline$\lambda$ & PM & URCS & SRCS & MRCS \\
\hline \multirow{2}{*}{2.0} & PDR & $0.843 \pm 0.006$ & $0.835 \pm 0.006$ & $0.847 \pm 0.009$ \\
\cline { 2 - 5 } & Delay & $0.740 \pm 0.003$ & $0.734 \pm 0.003$ & $0.740 \pm 0.005$ \\
\hline \multirow{2}{*}{2.5} & PDR & $0.822 \pm 0.007$ & $0.816 \pm 0.005$ & $0.814 \pm 0.004$ \\
\cline { 2 - 5 } & Delay & $0.731 \pm 0.003$ & $0.727 \pm 0.002$ & $0.728 \pm 0.002$ \\
\hline
\end{tabular}

\section{Surrogate Assisted EC-based FLC Design}

Since it is highly time-consuming to design FLCs by using EC techniques, we want to study the usefulness of surrogate models to improve the efficiency of the design process. In this research we have used MLP to build our surrogate model and performed experiments on both PSO and DE. We have considered different coding schemes, i.e. URCS, SRCS and MRCS, as well as three different settings for $(\eta)$. The results are reported in Table 4.4.

As depicted in Table 4.4, when $\lambda=2.0$, FLCs designed through surrogateassisted PSO with MRCS and SRCS (marked by ${ }^{\ddagger}$ ) can outperform FLCs that follow URCS. For example, when $\eta=50 \%$, one way ANOVA test and Tukey's post-hoc analysis report that, while their PDRs are indistinguishable, MRCS and SRCS provide significantly less delay than URCS $(\mathrm{p}<0.0001)$. On the other hand, if $\lambda=2.5$, designing FLCs through surrogate assisted PSO 
with URCS (marked by ${ }^{\ddagger}$ ) can outperform the other coding schemes. For instance, when $\lambda=2.5$ and $\eta=75 \%$, one way ANOVA test and Tukey's posthoc analysis show, while packet delay is indistinguishable, URCS provides significantly higher PDR $(\mathrm{p}<0.0001)$ than other coding schemes. Table 4.4 also presents the simulation results for surrogate-assisted DE. Statistical analysis indicates that no coding scheme is more effective in terms of both packet delay and PDR.

As mentioned in Subsection 4.2.4, in order to improve the accuracy of the surrogate model, in every iteration, the best $\eta$ individuals of the whole population are re-evaluated. Consistent with common intuition, we found that surrogate-assisted ECs with high $\eta$ (e.g. $\eta=75 \%$ ) are sometimes more effective. For example, if $\lambda=2.5$ and URCS is used, surrogate-assisted PSO with $\eta=75 \%$ offers significantly higher PDR than the results obtained when $\eta=25 \%$. Meanwhile, packet delay is indistinguishable. However, mostly no significant differences can be identified across the three different settings of $\eta$.

In view of the results reported in Tables 4.2, 4.3 and 4.4, we believe surrogate-assisted design can manage to achieve very good performance while significantly improving the efficiency of the design process as shown in Table 4.5. Although sometimes more effective FLCs can be identified without using surrogate models, surrogate-assisted PSO and DE can achieve more effective results than IEEE 802.15.4 and other competing algorithms (see Subsubsection 4.3.4). The simulation results also suggest that our fitness function with $\lambda=2.5$ can effectively guide the design of FLCs. Moreover, we have discovered that surrogate-assisted ECs with $\eta=25 \%$ are both efficient and effective. In view of the above and by further considering the need to design easily interpretable FLCs, we suggest that surrogate-assisted ECs with $\lambda=2.5, \eta=25 \%$ and MRCS (bolded in Table 4.4) present a suitable choice for practical use. 
Table 4.4: Results obtained by surrogate model for PSO and DE in terms of PDR and packet delay (seconds).

\begin{tabular}{|c|c|c|c|c|c|c|c|c|}
\hline \multirow{2}{*}{$\eta$} & & \multirow{2}{*}{ PM } & \multicolumn{3}{|c|}{ PSO } & \multicolumn{3}{|c|}{ DE } \\
\hline & & & URCS & SRCS & MRCS & URCS & SRCS & MRCS \\
\hline \multirow{4}{*}{$75 \%$} & \multirow[t]{2}{*}{2.0} & PDR & $\begin{array}{c}0.837 \\
\pm 0.014\end{array}$ & $\begin{array}{c}0.849 \\
\pm 0.006^{\ddagger}\end{array}$ & $\begin{array}{c}0.854 \\
\pm 0.011^{\ddagger}\end{array}$ & $\begin{array}{c}0.852 \\
\pm 0.008\end{array}$ & $\begin{array}{c}0.842 \\
\pm 0.013\end{array}$ & $\begin{array}{c}0.849 \\
\pm 0.015\end{array}$ \\
\hline & & Delay & $\begin{array}{c}0.777 \\
\pm 0.010\end{array}$ & $\begin{array}{c}0.727 \\
\pm 0.006^{\ddagger}\end{array}$ & $\begin{array}{c}0.740 \\
\pm 0.011^{\ddagger}\end{array}$ & $\begin{array}{c}0.745 \\
\pm 0.005\end{array}$ & $\begin{array}{c}0.758 \\
\pm 0.014\end{array}$ & $\begin{array}{c}0.769 \\
\pm 0.012\end{array}$ \\
\hline & \multirow[t]{2}{*}{2.5} & PDR & $\begin{array}{c}0.861 \\
\pm 0.003^{\ddagger \S}\end{array}$ & $\begin{array}{c}0.847 \\
\pm 0.003^{\S}\end{array}$ & $\begin{array}{c}0.851 \\
\pm 0.005^{\S}\end{array}$ & $\begin{array}{c}0.827 \\
\pm 0.006\end{array}$ & $\begin{array}{c}0.810 \\
\pm 0.004\end{array}$ & $\begin{array}{c}0.810 \\
\pm 0.005\end{array}$ \\
\hline & & Delay & $\begin{array}{c}0.719 \\
\pm 0.002^{\ddagger \S} \\
\end{array}$ & $\begin{array}{c}0.718 \\
\pm 0.002^{\S}\end{array}$ & $\begin{array}{c}0.719 \\
\pm 0.002^{\S}\end{array}$ & $\begin{array}{c}0.733 \\
\pm 0.003 \\
\end{array}$ & $\begin{array}{c}0.724 \\
\pm 0.002 \\
\end{array}$ & $\begin{array}{c}0.725 \\
\pm 0.002 \\
\end{array}$ \\
\hline \multirow{4}{*}{$50 \%$} & \multirow[t]{2}{*}{2.0} & PDR & $\begin{array}{c}0.846 \\
\pm 0.016\end{array}$ & $\begin{array}{c}0.847 \\
\pm 0.008^{\ddagger}\end{array}$ & $\begin{array}{c}0.848 \\
\pm 0.011^{\ddagger}\end{array}$ & $\begin{array}{c}0.849 \\
\pm 0.013\end{array}$ & $\begin{array}{c}0.831 \\
\pm 0.006 \\
\end{array}$ & $\begin{array}{c}0.841 \\
\pm 0.014 \\
\end{array}$ \\
\hline & & Delay & $\begin{array}{c}0.779 \\
\pm 0.010\end{array}$ & $\begin{array}{c}0.732 \\
\pm 0.008^{\ddagger}\end{array}$ & $\begin{array}{c}0.749 \\
\pm 0.012\end{array}$ & $\begin{array}{c}0.748 \\
\pm 0.009\end{array}$ & $\begin{array}{c}0.748 \\
\pm 0.007\end{array}$ & $\begin{array}{c}0.764 \\
\pm 0.008\end{array}$ \\
\hline & \multirow[t]{2}{*}{2.5} & PDR & $\begin{array}{c}0.856 \\
\pm 0.005^{\ddagger \S}\end{array}$ & $\begin{array}{c}0.849 \\
\pm 0.003\end{array}$ & $\begin{array}{c}0.855 \\
\pm 0.005^{\S}\end{array}$ & $\begin{array}{c}0.832 \\
\pm 0.006\end{array}$ & $\begin{array}{c}0.808 \\
\pm 0.004\end{array}$ & $\begin{array}{c}0.811 \\
\pm 0.005\end{array}$ \\
\hline & & Delay & $\begin{array}{c}0.722 \\
\pm 0.004^{\ddagger \S}\end{array}$ & $\begin{array}{c}0.721 \\
\pm 0.001^{\S}\end{array}$ & $\begin{array}{c}0.720 \\
\pm 0.002^{\S}\end{array}$ & $\begin{array}{c}0.736 \\
\pm 0.003\end{array}$ & $\begin{array}{c}0.724 \\
\pm 0.002\end{array}$ & $\begin{array}{c}0.727 \\
\pm 0.002 \\
\end{array}$ \\
\hline \multirow{4}{*}{$25 \%$} & \multirow[t]{2}{*}{2.0} & PDR & $\begin{array}{c}0.844 \\
\pm 0.015\end{array}$ & $\begin{array}{c}0.845 \\
\pm 0.008^{\ddagger}\end{array}$ & $\begin{array}{c}0.852 \\
\pm 0.013\end{array}$ & $\begin{array}{c}0.857 \\
\pm 0.013\end{array}$ & $\begin{array}{c}0.830 \\
\pm 0.009\end{array}$ & $\begin{array}{c}0.829 \\
\pm 0.011\end{array}$ \\
\hline & & Delay & $\begin{array}{c}0.760 \\
\pm 0.010\end{array}$ & $\begin{array}{c}0.725 \\
\pm 0.007^{\ddagger}\end{array}$ & $\begin{array}{c}0.750 \\
\pm 0.014\end{array}$ & $\begin{array}{c}0.757 \\
\pm 0.010\end{array}$ & $\begin{array}{c}0.748 \\
\pm 0.007\end{array}$ & $\begin{array}{c}0.754 \\
\pm 0.006\end{array}$ \\
\hline & \multirow[t]{2}{*}{2.5} & PDR & $\begin{array}{c}0.853 \\
\pm 0.004 \ddagger \S\end{array}$ & $\begin{array}{c}0.843 \\
\pm 0.004^{\S}\end{array}$ & $\begin{array}{c}0.844 \\
\pm 0.004^{\S}\end{array}$ & $\begin{array}{c}0.829 \\
\pm 0.006\end{array}$ & $\begin{array}{c}0.809 \\
\pm 0.007\end{array}$ & $\begin{array}{c}0.809 \\
\pm 0.007\end{array}$ \\
\hline & & Delay & $\begin{array}{c}0.719 \\
\pm 0.002\end{array}$ & $\begin{array}{c}0.720 \\
\pm 0.002^{\S}\end{array}$ & $\begin{array}{c}\mathbf{0 . 7 1 9} \\
\pm \mathbf{0 . 0 0 2}\end{array}$ & $\begin{array}{c}0.735 \\
\pm 0.003\end{array}$ & $\begin{array}{c}0.729 \\
\pm 0.002\end{array}$ & $\begin{array}{c}0.729 \\
\pm 0.003\end{array}$ \\
\hline
\end{tabular}

$\ddagger$ indicates coding schemes that outperform others indicates surrogate-assisted ECs that outperform EC techniques without using surrogate models 
Table 4.5: Average computation time of PSO and DE with our HPC facilities over 30 independent runs.

\begin{tabular}{|c|l|c|}
\hline \multicolumn{2}{|c|}{} & Time in Hours \\
\hline \multirow{4}{*}{ DE } & Without Surrogate Assistance & $119.782 \pm 0.289$ \\
\cline { 2 - 3 } & Surrogate Assisted (75\%) & $93.308 \pm 0.683$ \\
\cline { 2 - 3 } & Surrogate Assisted (50\%) & $66.903 \pm 2.311$ \\
\cline { 2 - 3 } & Surrogate Assisted (25\%) & $30.481 \pm 0.283$ \\
\hline \multirow{4}{*}{ PSO } & Without Surrogate Assistance & $118.937 \pm 0.736$ \\
\cline { 2 - 3 } & Surrogate Assisted (75\%) & $92.642 \pm 0.947$ \\
\cline { 2 - 3 } & Surrogate Assisted (50\%) & $65.457 \pm 5.271$ \\
\cline { 2 - 3 } & Surrogate Assisted (25\%) & $30.173 \pm 0.491$ \\
\hline
\end{tabular}

Comparison between EC-based FLC Design (Target $\mathrm{T}_{1}$ ) and Competing Algorithms

In order to measure the effectiveness of our automatic FLC design, we compare it with recent competing algorithms, i.e. IEEE 802.15.4, ACS, $\mathrm{D}^{2} \mathrm{MAC}$ and NB-step. For a good compromise between effectiveness and interpretability as we mentioned in Subsection 4.2.1, surrogate-assisted PSO design with $\lambda=2.5, \eta=25 \%$ and MRCS are compared with these competing algorithms.

As presented in Table 4.6, $\mathrm{CLFB}_{T_{1}}$ outperforms IEEE 802.15.4, ACS, $\mathrm{D}^{2} \mathrm{MAC}$ and NB-step in terms of both PDR and packet delay. The one-way ANOVAs give significant differences with $p$-value $<0.0001$. The corresponding Tukey's post-hoc analysis further confirms our conclusion. Specifically, PDR achieved by $\mathrm{CLFB}_{T_{1}}$ is significantly higher than IEEE 802.15.4, ACS, $\mathrm{D}^{2} \mathrm{MAC}$ and NB-step without any negative impact on packet delay.

As mentioned in Subsection 4.2.2, the other design objective is to improve throughput which is realised implicitly by enhancing PDR. Table 4.6 further reports WBAN throughput upon using each algorithm. Statistical analysis confirms that $\mathrm{CLFB}_{T_{1}}$ achieves significantly higher throughput 
than the other algorithms. Moreover, $\mathrm{CLFB}_{T_{1}}$ can significantly reduce the collision rate in comparison to all competing algorithms.

Table 4.6: Results obtained by competing algorithms, i.e. IEEE 802.15.4, $\mathrm{D}^{2} \mathrm{MAC}$ and NB-step.

\begin{tabular}{|c|c|c|c|c|}
\hline & PDR & Delay (seconds) & Throughput (bps) & Collision rate \\
\hline IEEE 802.15.4 & $0.751 \pm 0.003$ & $0.725 \pm 0.003$ & $1925.678 \pm 11.150$ & $0.370 \pm 0.003$ \\
\hline ACS & $0.691 \pm 0.002$ & $\mathbf{0 . 6 8 1} \pm \mathbf{0 . 0 0 3}$ & $1783.331 \pm 10.988$ & $0.410 \pm 0.002$ \\
\hline D $^{2}$ MAC & $0.826 \pm 0.003$ & $0.760 \pm 0.002$ & $2101.920 \pm 12.496$ & $0.329 \pm 0.002$ \\
\hline NB-step & $0.825 \pm 0.002$ & $0.760 \pm 0.003$ & $2098.524 \pm 12.114$ & $0.329 \pm 0.002$ \\
\hline CLFB $_{T_{1}}$ & $\mathbf{0 . 8 4 4} \pm \mathbf{0 . 0 0 4}$ & $\mathbf{0 . 7 1 9} \pm \mathbf{0 . 0 0 2}$ & $\mathbf{2 2 0 9 . 7 7 6} \pm \mathbf{1 4 . 9 6 6}$ & $\mathbf{0 . 2 7 1} \pm \mathbf{0 . 0 0 4}$ \\
\hline
\end{tabular}

\section{Summary of Automatic Design of FLCs for Target $T_{1}$}

In this subsection, we have succeeded in (1) effectively designing FLCs based on ECs for target $T_{1}$ and (2) improving significantly the efficiency of the design process with the help of surrogate models. Although our statistical analysis suggests that PSO can sometimes slightly outperform DE, it has been shown that both of them can be effectively utilised to design FLCs. We have also studied the usefulness of three coding schemes, i.e. URCS, SRCS and MRCS, and concluded that, although URCS is generally more effective than MRCS, FLC designs through MRCS are more interpretable. Moreover, We have showed that with $\eta=25 \%$, the surrogate model can improve the efficiency of the design process significantly while keeping the accuracy at an acceptable level. We have also demonstrated that the fitness function with $\lambda=2.5$ should be employed to satisfy both the reliability and performance requirements in WBANs. Finally, we statistically confirmed the effectiveness of $\mathrm{CLFB}_{T_{1}}$ by comparing it to other competing algorithms. The results show that sensor nodes using $\mathrm{CLFB}_{T_{1}}$ can provide significantly better reliability and performance than competing algorithms such as IEEE 802.15.4, ACS, D²MAC and NB-step. 
Due to improvements in effectiveness as mentioned above, we believe that the time spent on designing FLCs through our approach is worthwhile. Moreover, once $\mathrm{CLFB}_{T_{1}}$ is designed, it can be easily embedded into each sensor node and repeatedly used for different WBAN applications.

\subsubsection{Automatic Design of FLCs for Multiple Network Set- tings (Target $\mathbf{T}_{2}$ )}

In this subsection WBAN simulation settings for design target $\mathrm{T}_{2}$ will be explained first. Accordingly, we statistically study the effectiveness of different coding schemes with respect to both ECs and surrogate-assisted ECs. Finally, the reliability and performance of $\mathrm{CLFB}_{T_{2}}$ are compared with the competing algorithms.

\section{Network Simulation Scenario for Target $\mathbf{T}_{2}$}

FLC designs for target $T_{1}$ may not produce a desirable balance between reliability and performance over a wide range of WBAN settings. Therefore, as explained in Subsection 4.2.3, we also have the aim to design FLCs based on target $\mathrm{T}_{2}$ (i.e. enhancing reliability and performance over multiple WBAN's settings). For this purpose, we have built separate training and testing scenarios. In particular, five different network settings described in Table 4.7 are used for training. Again, Table 4.1 is used to determine the communication features of each sensor node. Table 4.8 summarises all testing scenarios for this study. Specifically, to test the generalisability of FLC designs, we have considered up to eight different sensors during testing. All these sensors are commonly studied in the literature [2, 119]. Meanwhile, two ECG sensors are included in testing scenario 9 in Table 4.8 in order to examine WBAN with high traffic load. 
Table 4.7: WBAN settings for designing FLCs regarding target $\mathrm{T}_{2}$.

\begin{tabular}{|c|l|l|}
\hline Scenario & No of sensors & WBAN setting \\
\hline 1 & Three sensors & Temperature, ECG, respiratory rate \\
\hline 2 & Four sensors & Temperature, ECG, respiratory rate and HR \\
\hline 3 & Six sensors & $\begin{array}{l}\text { Temperature, ECG, EEG, respiratory rate, HR } \\
\text { and BP }\end{array}$ \\
\hline 4 & Seven sensors & $\begin{array}{l}\text { Temperature, ECG, EEG, respiratory rate, HR, BP } \\
\text { and Motion }\end{array}$ \\
\hline 5 & Eight sensors & $\begin{array}{l}\text { Temperature, ECG, EEG, respiratory rate, HR, BP, } \\
\text { Motion and Endoscope Imaging }\end{array}$ \\
\hline
\end{tabular}

Table 4.8: WBAN settings for testing FLCs designed regarding target $\mathrm{T}_{2}$.

\begin{tabular}{|c|l|l|}
\hline Scenario & No of sensors & WBAN setting \\
\hline 1 & One sensor & Temperature \\
\hline 2 & Two sensors & Temperature and ECG \\
\hline 3 & Three sensors & Temperature, ECG and respiratory rate \\
\hline 4 & Four sensors & Temperature, ECG, respiratory rate and HR \\
\hline 5 & Five sensors & Temperature, ECG, respiratory rate, HR and EEG \\
\hline 6 & Six sensors & $\begin{array}{l}\text { Temperature, ECG, EEG, respiratory rate, HR, } \\
\text { and BP }\end{array}$ \\
\hline 7 & Seven sensors & $\begin{array}{l}\text { Temperature, ECG, EEG, respiratory rate, HR, BP } \\
\text { and Motion }\end{array}$ \\
\hline 8 & Eight sensors & $\begin{array}{l}\text { Temperature, ECG, EEG, respiratory rate, HR, BP, } \\
\text { Motion and Endoscope Imaging }\end{array}$ \\
\hline 9 & Nine sensors & $\begin{array}{l}\text { Temperature, ECG, EEG, respiratory rate, HR, BP, } \\
\text { Motion, Endoscope Imaging and ECG }\end{array}$ \\
\hline
\end{tabular}




\section{PSO-based and Surrogate-Assisted PSO-based FLC Design}

Since Subsection 4.3.4 confirms that both PSO and DE are effective for designing FLCs, we decide to only use PSO to design FLCs for target $\mathrm{T}_{2}$. Subsequently, the training results from PSO for the three different coding schemes, i.e. URCS, SRCS and MRCS, are presented in Table 4.9. From the results, we found that the differences among the three coding schemes generally are statistically indistinguishable. However, in training scenarios seven and eight, PSO with URCS outperforms other coding schemes (improved PDR with compatible packet delay).

In Subsection 4.3.4, we have shown that surrogate-assisted ECs with $\eta=25 \%$ are efficient and effective for designing FLCs. Consequently, we also examine surrogate-assisted PSO with $\eta=25 \%$ for design target $\mathrm{T}_{2}$. The training results presented in Table 4.9 clearly show that there is no significant difference between PSO and surrogate-assisted PSO in terms of effectiveness, regardless of the coding scheme. On the other hand, the computation time for designing FLC through PSO and surrogate-assisted $\mathrm{PSO}$ is $491.96 \pm 5.42$ and $148.30 \pm 7.47$ hours, respectively. Since there is an almost $70 \%$ saving of computation time upon using surrogate-assisted PSO and it is as effective as PSO, we think the best choice again is to design FLCs through surrogate-assisted PSO. It should be mentioned that the computation time for designing FLCs is totally worthwhile since the designed FLCs can be repeatedly used in many WBAN applications, and enhance the effectiveness of the network significantly.

\section{Comparison between PSO-based FLC Design (Target $\mathrm{T}_{2}$ ) and Compet- ing Algorithms}

After comparing $\mathrm{CLFB}_{T_{2}}$ with competing algorithms including $\mathrm{CLFB}_{T_{1}}$ on the testing results (see Tables 4.10, 4.11, 4.12 and 4.13), we found that only $\mathrm{CLFB}_{T_{2}}$ can clearly outperform IEEE 802.15.4 in both PDR and packet delay. In other words, $\mathrm{CLFB}_{T_{2}}$ is the only algorithm which can provide higher 
Table 4.9: Training results obtained by PSO and surrogate-assisted PSO in terms of PDR and packet delay in seconds $(\lambda=2.5)$.

\begin{tabular}{|c|c|c|c|c|c|c|c|}
\hline \multirow{2}{*}{$\begin{array}{l}\text { Training } \\
\text { Scenario }\end{array}$} & \multirow{2}{*}{ PM } & \multicolumn{3}{|c|}{ PSO } & \multicolumn{3}{|c|}{ Surrogate-Assisted PSO } \\
\hline & & URCS & SRCS & MRCS & URCS & SRCS & MRCS \\
\hline \multirow{4}{*}{1} & PDP & 0.844 & 0.844 & 0.844 & 0.844 & 0.841 & 0.843 \\
\hline & ID & \pm 0.002 & \pm 0.002 & \pm 0.002 & \pm 0.002 & \pm 0.002 & \pm 0.001 \\
\hline & Dolay & 0.260 & 0.260 & 0.260 & 0.260 & 0.258 & 0.259 \\
\hline & Delay & \pm 0.001 & \pm 0.001 & \pm 0.001 & \pm 0.001 & \pm 0.001 & \pm 0.001 \\
\hline \multirow{4}{*}{2} & מחס & 0.860 & 0.856 & 0.860 & 0.860 & 0.849 & 0.852 \\
\hline & IDI & \pm 0.001 & \pm 0.004 & \pm 0.001 & \pm 0.001 & \pm 0.004 & \pm 0.004 \\
\hline & Dolo & 0.271 & 0.270 & 0.272 & 0.272 & 0.266 & 0.268 \\
\hline & Deray & \pm 0.001 & \pm 0.002 & \pm 0.001 & \pm 0.001 & \pm 0.003 & \pm 0.003 \\
\hline \multirow{4}{*}{3} & PDR & 0.519 & 0.512 & 0.512 & 0.514 & 0.511 & 0.512 \\
\hline & ID & \pm 0.002 & \pm 0.001 & \pm 0.001 & \pm 0.002 & \pm 0.001 & \pm 0.002 \\
\hline & & 0.303 & 0.298 & 0.299 & 0.301 & 0.299 & 0.299 \\
\hline & Delay & \pm 0.002 & \pm 0.001 & \pm 0.002 & \pm 0.002 & \pm 0.002 & \pm 0.002 \\
\hline \multirow{4}{*}{4} & $\mathrm{D}$ & 0.489 & 0.485 & 0.484 & 0.485 & 0.484 & 0.485 \\
\hline & IDN & \pm 0.002 & \pm 0.001 & \pm 0.001 & \pm 0.002 & \pm 0.001 & \pm 0.002 \\
\hline & Delay & 0.309 & 0.308 & 0.308 & 0.308 & 0.310 & 0.311 \\
\hline & Deray & \pm 0.001 & \pm 0.002 & \pm 0.001 & \pm 0.001 & \pm 0.002 & \pm 0.002 \\
\hline \multirow{4}{*}{5} & PDR & 0.474 & 0.471 & 0.471 & 0.471 & 0.471 & 0.471 \\
\hline & I & \pm 0.002 & \pm 0.001 & \pm 0.001 & \pm 0.002 & \pm 0.002 & \pm 0.002 \\
\hline & & 0.308 & 0.309 & 0.311 & 0.309 & 0.313 & 0.313 \\
\hline & Delay & \pm 0.002 & \pm 0.002 & \pm 0.002 & \pm 0.003 & \pm 0.004 & \pm 0.003 \\
\hline
\end{tabular}


PDR and throughput than IEEE 802.15.4 without incurring longer delay. Moreover, it produces less collision rate than IEEE 802.15.4. Although $\mathrm{CLFB}_{T_{2}}$ can outperform other competing algorithms from this perspective, it does not always produce the highest level of reliability, however.

Table 4.10 shows that PDR is almost $100 \%$ when the network consists of only two nodes including the coordinator. When the traffic load increases by adding more sensor nodes, PDR starts to decrease. Table 4.10 shows that $\mathrm{CLFB}_{T_{1}}, \mathrm{D}^{2} \mathrm{MAC}$ and NB-step can manage to achieve higher PDR than $\mathrm{CLFB}_{T_{2}}$ in some scenarios (e.g. scenarios 3,4 and 5). This achieved by imposing significantly longer backoffs. On the other hand, the results clearly show $\mathrm{CLFB}_{T_{2}}$ is more reliable than IEEE 802.15.4 and ACS without imposing longer backoffs on sensor nodes.

Generally, by increasing the number of nodes, PDR will decrease in all algorithms. This is because of the increasing number of collisions on the network, as shown in Table 4.11. In addition, $\mathrm{CLFB}_{T_{1}}, \mathrm{D}^{2} \mathrm{MAC}$ and NB-step can achieve less collision rate than $\mathrm{CLFB}_{T_{2}}$. However, they force the sensor nodes to increase their backoff delay to avoid collisions. On the other hand, $\mathrm{CLFB}_{T_{2}}$ can perform much better than IEEE 802.15.4 and ACS in term of collision rate without sacrificing communication delay.

As mentioned before, MAC throughput of the network presented in Table 4.12 is indirectly improved by increasing PDR in WBANs. Consequently, $\mathrm{CLFB}_{T_{1}}, \mathrm{D}^{2} \mathrm{MAC}$ and NB-step can outperform $\mathrm{CLFB}_{T_{2}}$ in terms of throughput in some scenarios (e.g. scenarios 3, 4 and 5). Nevertheless, our statistical analysis shows that $\mathrm{CLFB}_{T_{2}}$ statistically outperforms IEEE 802.15.4 and ACS when there are more than two sensor nodes in the network.

Although the results show $\mathrm{CLFB}_{T_{1}}, \mathrm{D}^{2} \mathrm{MAC}$ and NB-step can sometimes achieve higher PDR and throughput than $\mathrm{CLFB}_{T_{2}}$, it is important to note that they sacrifice the packet delay hugely which means they are undesirable for delay sensitive WBAN applications. For example, in scenario 7, $\mathrm{CLFB}_{T_{1}}, \mathrm{D}^{2} \mathrm{MAC}$ and NB-step introduce significantly longer delay 


\begin{tabular}{|c|c|c|c|c|c|c|}
\hline$a$ & \begin{tabular}{ll}
$\stackrel{+}{+}$ & $\overline{8}$ \\
\multirow{2}{*}{} & 0 \\
0 & 0
\end{tabular} & 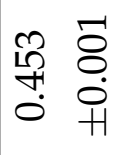 & 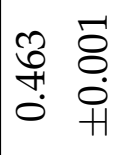 & \begin{tabular}{ll}
$\hat{1}$ & $\overline{8}$ \\
\multirow{2}{*}{} & 0 \\
0 & 0 \\
0 & +1
\end{tabular} & $\mid \begin{array}{ll}8 & \overline{8} \\
10 & 8 \\
0 & 0 \\
0 & +1\end{array}$ & $\begin{array}{ll}m & \overline{8} \\
\infty & 8 \\
+1 & 0 \\
0 & +\end{array}$ \\
\hline$\infty$ & 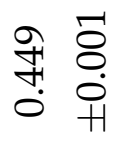 & 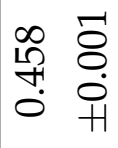 & $\begin{array}{ll}12 & 5 \\
0 & 8 \\
+1 & 0 \\
0 & +1\end{array}$ & $\begin{array}{ll}7 & 5 \\
0 & 8 \\
+1 & 0 \\
0 & +1\end{array}$ & $\begin{array}{ll}0 & 5 \\
0 & 8 \\
10 & 0 \\
0 & +\end{array}$ & \begin{tabular}{ll}
8 & 8 \\
\multirow{2}{*}{} & 8 \\
0 & 0 \\
0 & 0
\end{tabular} \\
\hline$\Lambda$ & \begin{tabular}{ll}
$\Re$ & $\overline{8}$ \\
\multirow{f}{*}{} & 0 \\
0 & 0 \\
0 & +1
\end{tabular} & 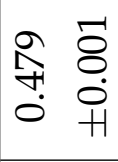 & 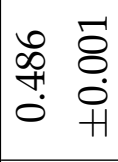 & 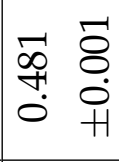 & 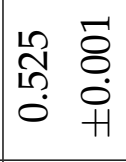 & $\begin{array}{ll}1 & - \\
0 & 8 \\
10 & 0 \\
0 & +\end{array}$ \\
\hline 6 & 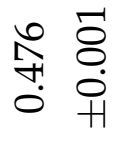 & 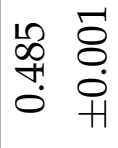 & $\begin{array}{ll}0 & \overline{8} \\
10 & 8 \\
0 & 0 \\
0 & +1\end{array}$ & $\begin{array}{ll}\overrightarrow{7} & \overline{8} \\
10 & 0 \\
0 & 0 \\
0 & +1\end{array}$ & $\mid \begin{array}{ll}n & 5 \\
10 & 8 \\
10 & 0 \\
0 & +\end{array}$ & $\begin{array}{ll}\sim & \overline{8} \\
\text { in } & 0 \\
0 & 0 \\
0 & +\end{array}$ \\
\hline n & 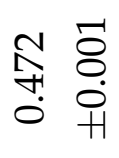 & \begin{tabular}{ll}
8 & $\overline{8}$ \\
$\infty$ & 8 \\
\multirow{+}{+1}{} & 0 \\
0 & +1
\end{tabular} & 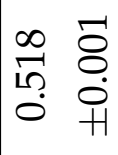 & 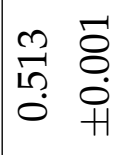 & $\begin{array}{ll}10 & 5 \\
10 & 8 \\
10 & 0 \\
0 & +1\end{array}$ & $\begin{array}{ll}\infty & -1 \\
0 & 8 \\
10 & 0 \\
0 & +\end{array}$ \\
\hline H & $\begin{array}{ll}\overrightarrow{0} & \overline{8} \\
\infty & 0 \\
0 & 0 \\
0 & +\end{array}$ & $\begin{array}{ll}0 & \overline{8} \\
0 & 0 \\
0 & 0 \\
0 & +1\end{array}$ & $\begin{array}{ll}\infty & \overline{8} \\
\infty & 8 \\
\infty & 0 \\
0 & 0 \\
0 & +1\end{array}$ & $\begin{array}{ll}0 & 5 \\
\infty & 8 \\
\infty & 0 \\
0 & 0 \\
0 & +1\end{array}$ & $\begin{array}{ll}N & \overline{8} \\
\infty & 8 \\
0 & 0 \\
0 & +\end{array}$ & $\begin{array}{ll}0 & \overline{8} \\
0 & 8 \\
\infty & 0 \\
0 & +\end{array}$ \\
\hline$n$ & $\begin{array}{ll}0 & \overline{8} \\
\infty & 8 \\
0 & 0 \\
0 & +\end{array}$ & 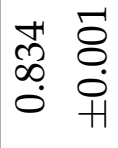 & \begin{tabular}{ll}
$\infty$ & \multicolumn{1}{c}{} \\
$\infty$ & 0 \\
$\infty$ & 0 \\
0 & +
\end{tabular} & $\begin{array}{ll}10 & \overline{8} \\
\infty & 0 \\
\infty & 0 \\
0 & +\end{array}$ & 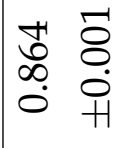 & 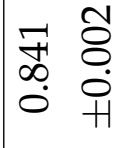 \\
\hline$N$ & 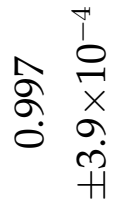 & 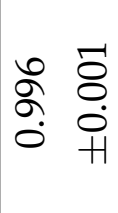 & 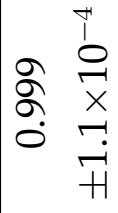 & 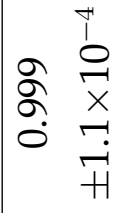 & $\begin{array}{ll}\hat{\sigma} & \overline{8} \\
\sigma & \circ \\
0 & 0 \\
0 & +\end{array}$ & 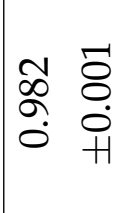 \\
\hline$r$ & \begin{tabular}{ll}
8 & 8 \\
8 & 8 \\
\hdashline & 0 \\
- & +1
\end{tabular} & $\begin{array}{ll}8 & 8 \\
\varnothing & 8 \\
- & 0 \\
-1\end{array}$ & 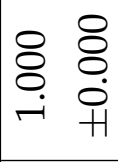 & $\begin{array}{ll}8 & 8 \\
8 & 8 \\
- & \stackrel{+}{+}\end{array}$ & \begin{tabular}{ll}
8 & 8 \\
8 & 8 \\
\hdashline & 0 \\
- & 0
\end{tabular} & \begin{tabular}{ll}
8 & 8 \\
8 & 8 \\
\hdashline & $\stackrel{0}{+1}$
\end{tabular} \\
\hline 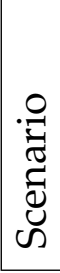 & 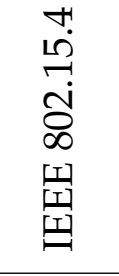 & $\int_{4}^{\infty}$ & 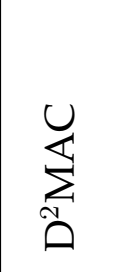 & $\begin{array}{l}\frac{0}{0} \\
\dot{p} \\
n \\
n^{2}\end{array}$ & 包 & $\stackrel{\hat{N}^{N}}{\tilde{U}}$ \\
\hline
\end{tabular}




\begin{tabular}{|c|c|c|c|c|c|c|c|}
\hline 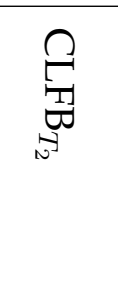 & $\underset{7}{7}$ & 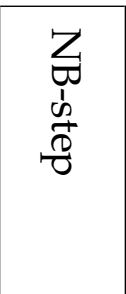 & $\begin{array}{l}\nabla_{N} \\
\sum^{3} \\
3\end{array}$ & () & & 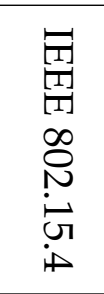 & 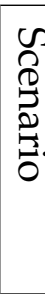 \\
\hline $\begin{array}{ll}+ & 0 \\
0 & 0 \\
8 & 8 \\
8 & 8\end{array}$ & $\begin{array}{ll} & 0 \\
0 & 8 \\
8 & 8 \\
8 & 8\end{array}$ & $\begin{array}{ll} & 0 \\
\circ & \circ \\
8 & 8 \\
8 & 8\end{array}$ & $\begin{array}{ll}+ & 0 \\
0 & 8 \\
8 & 8 \\
8 & 8\end{array}$ & $\begin{array}{l}+ \\
0 \\
8 \\
8 \\
8\end{array}$ & & $\begin{array}{l}14 \\
\stackrel{0}{8} \\
8 \\
8\end{array}$ & $\mapsto$ \\
\hline 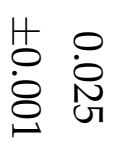 & $\begin{array}{ll}H & 0 \\
0 & 0 \\
8 & N \\
\wp & 0\end{array}$ & $\begin{array}{ll}+ & 0 \\
0 & 0 \\
\odot & \wp \\
\ominus & \infty\end{array}$ & 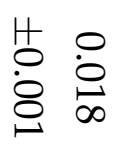 & $\begin{array}{ll}1 & 5 \\
0 & 0 \\
8 & 1 \\
8\end{array}$ & & $\begin{array}{ll} & + \\
0 & \circ \\
8 & 0 \\
0 & 0\end{array}$ & $N$ \\
\hline 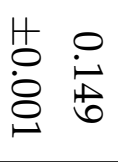 & 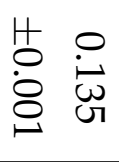 & 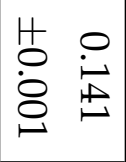 & 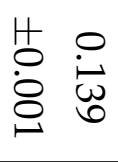 & 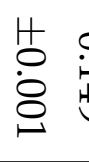 & 苨 & 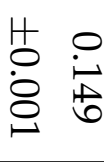 & $\omega$ \\
\hline 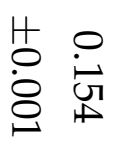 & $\begin{array}{l}\stackrel{+}{\circ} \stackrel{\circ}{\ominus} \\
\stackrel{\omega}{\ominus}\end{array}$ & 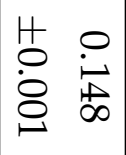 & $\begin{array}{ll}+ & 0 \\
\circ & 0 \\
8 & \text { 占 }\end{array}$ & $\begin{array}{l}1+ \\
8 \\
8 \\
8\end{array}$ & & 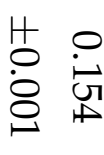 & $\mathbb{A}$ \\
\hline 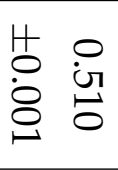 & 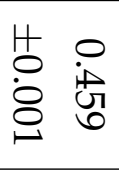 & $\begin{array}{ll} & 0 \\
0 & 0 \\
\varnothing & \oplus \\
\ominus & \oplus\end{array}$ & 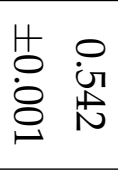 & $\begin{array}{l}+ \\
0 \\
8 \\
\varnothing\end{array}$ & $\begin{array}{l}0 \\
\stackrel{i}{i} \\
\omega \\
\omega\end{array}$ & 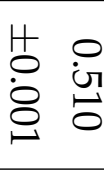 & $v$ \\
\hline 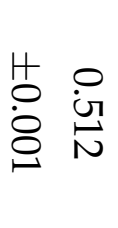 & $\begin{array}{ll}+ & \circ \\
0 & \circ \\
8 & 8 \\
\ominus & 8\end{array}$ & \begin{tabular}{ll}
+ & 0 \\
0 & 0 \\
8 & \multirow{1}{N}{} \\
$\ominus$ & 1
\end{tabular} & $\begin{array}{ll}+ & 0 \\
0 & 0 \\
8 & 0 \\
\ominus & \oplus\end{array}$ & 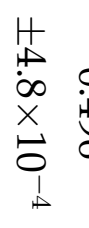 & $\begin{array}{l}0 \\
0 \\
0\end{array}$ & 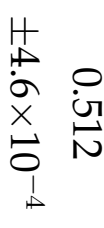 & $\sigma$ \\
\hline 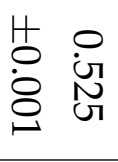 & 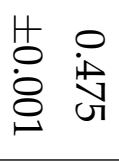 & $\begin{array}{ll}+ & 0 \\
0 & 0 \\
8 & 8 \\
\circ & 8\end{array}$ & $\begin{array}{ll}+ & 0 \\
0 & 0 \\
8 & 0 \\
\circ & 8\end{array}$ & $\begin{array}{l}+ \\
0 \\
\varnothing \\
\ominus\end{array}$ & 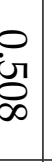 & $\begin{array}{ll} & + \\
0 & 0 \\
\text { in } & \text { ज }\end{array}$ & $V$ \\
\hline 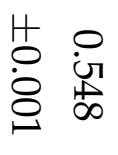 & 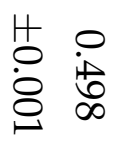 & 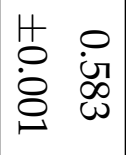 & 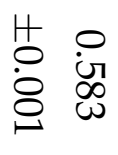 & \begin{tabular}{ll} 
& + \\
0 & \multicolumn{1}{c}{} \\
8 & 1 \\
0 & 1
\end{tabular} & : & 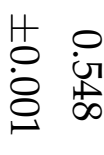 & $\infty$ \\
\hline 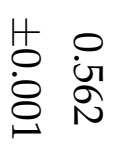 & $\begin{array}{ll} & 0 \\
0 & 0 \\
\text { ஸึ } & \end{array}$ & $\begin{array}{ll}+ & 0 \\
0 & \text { نु } \\
8 & 0 \\
\wp & N\end{array}$ & 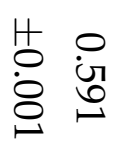 & $\begin{array}{l}+4 \\
0 \\
8 \\
8 \\
\end{array}$ & $\begin{array}{l}\circ \\
\dot{0} \\
\notin\end{array}$ & 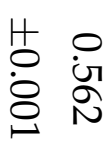 & 6 \\
\hline
\end{tabular}




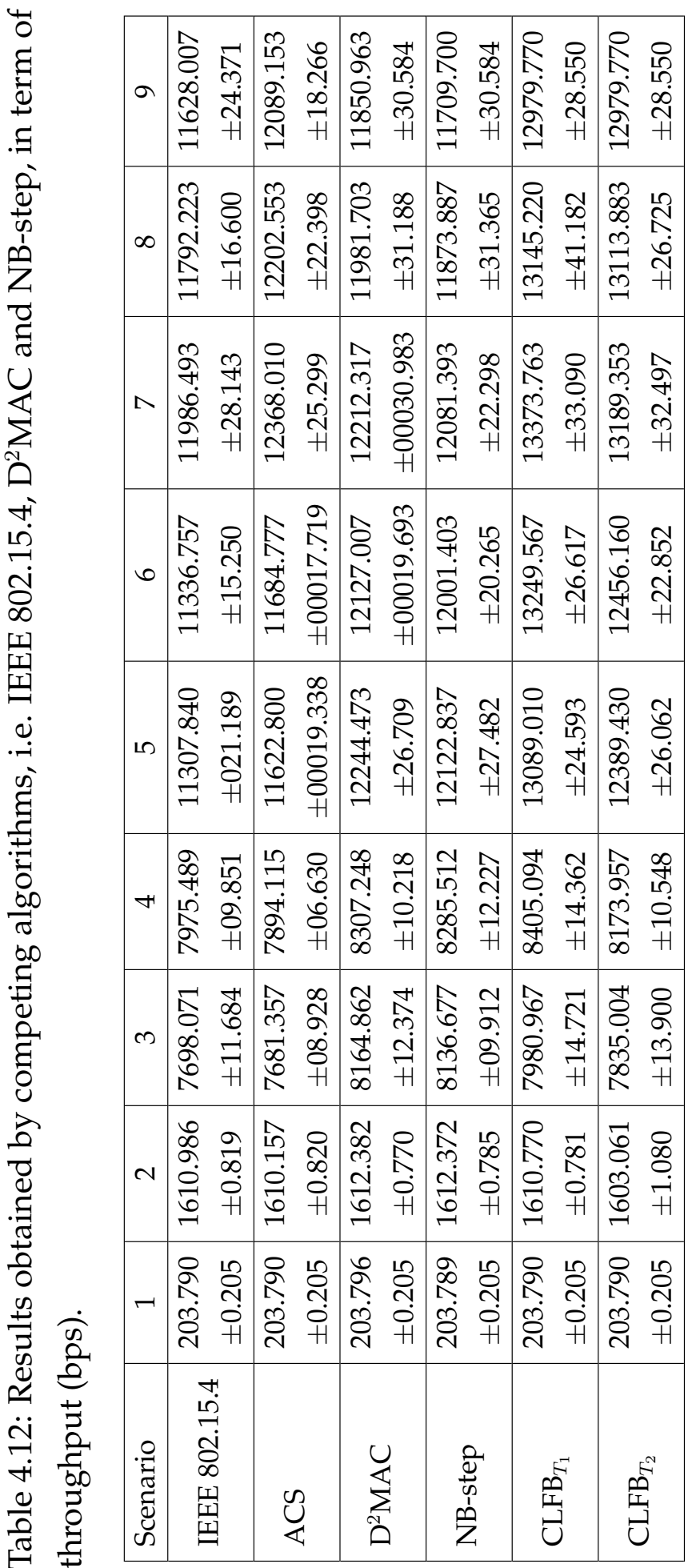


than IEEE 802.15.4, as shown in Table 4.13. Unlike the competing approaches, $\mathrm{CLFB}_{T_{2}}$ can keep the packet delay close to the level obtained in IEEE 802.15.4. Particularly, ANOVA and Tukey's post-hoc analysis indicate that the delay achieved by $\mathrm{CLFB}_{T_{2}}$ is statistically indistinguishable from that of IEEE 802.15.4 and ACS. Moreover, $\mathrm{CLFB}_{T_{2}}$ can manage to achieve significantly less delay than $\mathrm{CLFB}_{T_{1}}, \mathrm{D}^{2} \mathrm{MAC}$ and NB-step.

\section{Summary of Automatic Design of FLCs for Target $\mathrm{T}_{2}$}

In this subsection, we have successfully designed FLCs for target $T_{2}$ by using both PSO and surrogate-assisted PSO. We found that surrogateassisted PSO is more desirable in view of both efficiency and effectiveness. Our simulation results further revealed the main strength of $\mathrm{CLFB}_{T_{2}}$ which is its effectiveness. These results also demonstrated that $\mathrm{CLFB}_{T_{2}}$ is the only method that can significantly outperform IEEE 802.15.4 concerning both reliability and performance. It is worthwhile to note that, besides its effectiveness and efficiency, $\mathrm{CLFB}_{T_{2}}$ also provides general applicability. This means that during practical use, patients can add or remove any specific sensor nodes and the network remains effective.

\subsection{Chapter Summary}

In this chapter, we have proposed an EC-based approach for fully automated design of Fuzzy Logic Controllers (FLCs) for Cross-Layer Fuzzy Logic based Backoff (CLFB) in WBANs. In order to design FLCs effectively, we have studied the influence of three different coding schemes on the effectiveness and interpretability of the FLCs designed. This chapter also defined a fitness function with aim of achieving high network reliability and performance in WBANs with respect to two separate design targets. The first target is designing effective FLCs (i.e. $\mathrm{CLFB}_{T_{1}}$ ) to provide reliability and performance for a specific WBAN configuration. On the other hand, the second target aims to design desirable FLCs (i.e. CLFB ${ }_{T_{2}}$ ) for 


\begin{tabular}{|c|c|c|c|c|c|}
\hline 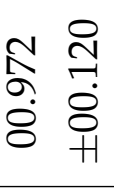 & 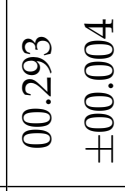 & 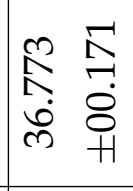 & 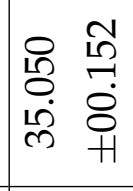 & 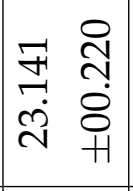 & 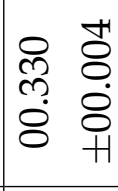 \\
\hline 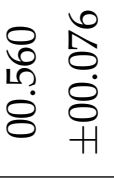 & 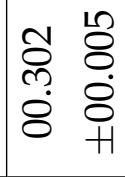 & 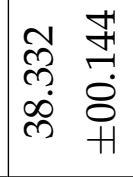 & 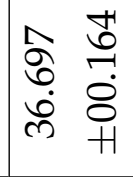 & 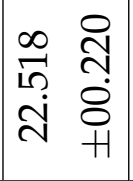 & 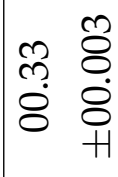 \\
\hline 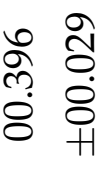 & 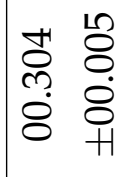 & 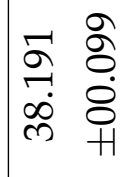 & 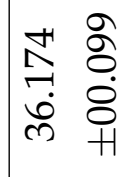 & 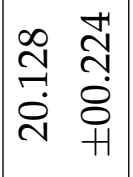 & 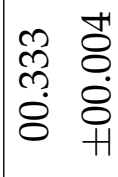 \\
\hline 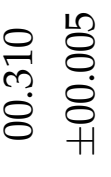 & 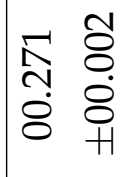 & 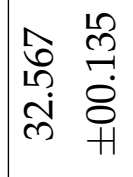 & 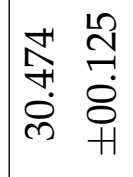 & 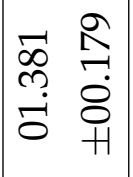 & 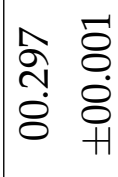 \\
\hline 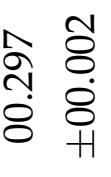 & $\begin{array}{ll}0 & 0 \\
0 & 8 \\
\text { ํ} & 0 \\
\dot{8} & 8 \\
0 & +1\end{array}$ & 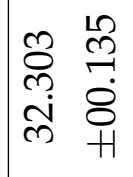 & 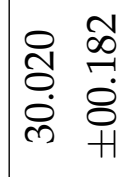 & 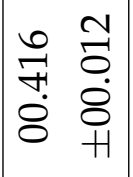 & 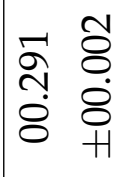 \\
\hline 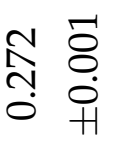 & $\begin{array}{ll}\hat{N} & \overline{8} \\
\stackrel{0}{0} & 0 \\
0 & +\end{array}$ & $\begin{array}{ll}0 & \overrightarrow{8} \\
\grave{0} & \stackrel{8}{0} \\
0 & 0\end{array}$ & 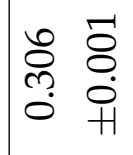 & $\left|\begin{array}{cc}-1 & 5 \\
0 & 8 \\
0 & 0 \\
0 & 0 \\
0 & H\end{array}\right|$ & 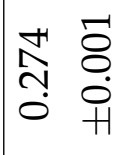 \\
\hline 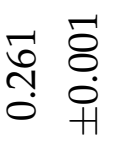 & $\begin{array}{ll}\stackrel{n}{0} & \overline{8} \\
& 0 \\
0 & 0\end{array}$ & \begin{tabular}{ll}
0 & \multicolumn{1}{c}{} \\
$\dddot{\theta}$ & 0 \\
0 & 0 \\
0 & +
\end{tabular} & 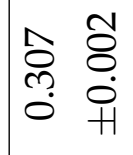 & $\left|\begin{array}{ll}0 & -1 \\
0 & 0 \\
& 0 \\
0 & +1\end{array}\right|$ & 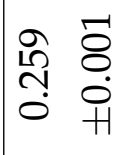 \\
\hline 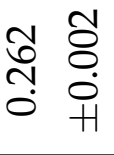 & 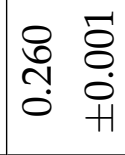 & 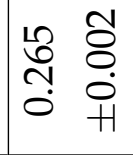 & 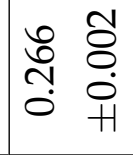 & 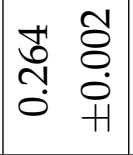 & 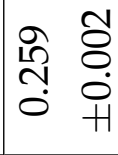 \\
\hline 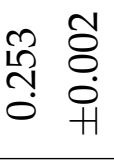 & 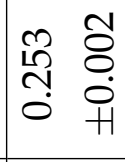 & $\begin{array}{ll}\hat{N} & \overline{8} \\
& 0 \\
0 & +\end{array}$ & 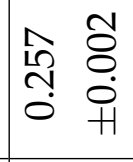 & $\left|\begin{array}{ll}0 & -1 \\
\stackrel{0}{0} & 0 \\
0 & 0 \\
0 & +1\end{array}\right|$ & 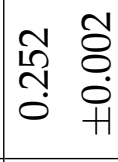 \\
\hline 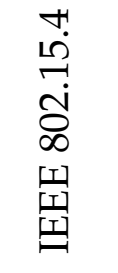 & $\int_{4}^{\infty}$ & 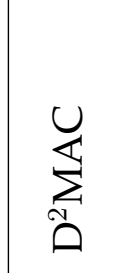 & $\begin{array}{l}\frac{0}{0} \\
\frac{1}{p} \\
n \\
Z\end{array}$ & $\stackrel{\tilde{D}^{-1}}{u}$ & 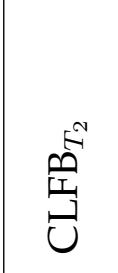 \\
\hline
\end{tabular}


more general WBAN settings.

To achieve our design targets, we have successfully utilised surrogate models to improve the efficiency of the design process. Moreover, we have examined the usefulness of our approach with the help of two wellknown EC techniques, i.e. Particle Swarm Optimization (PSO) and Differential Evolutionary (DE). We have shown that PSO slightly outperformed DE for our design problems. Moreover, we have confirmed that $\mathrm{CLFB}_{T_{1}}$ can achieve significantly better reliability and performance than IEEE 802.15.4, ACS, D 2 MAC and NB-step. We have also experimentally shown that only $\mathrm{CLFB}_{T_{2}}$ can outperform IEEE 802.15.4 in terms of both PDR and packet delay for a wider range of communication scenarios.

It is worthwhile mentioning that, during the whole design process, we have introduced neither specific mechanism nor significant changes to IEEE 802.15.4. FLCs are fuzzy rules that are simple and easy to process. Once FLCs for CLFB have been designed through our automatic approach, they can be easily integrated into sensor nodes as long as they are compatible with IEEE 802.15.4 without any major changes.

As mentioned before, we have primarily focused on Carrier Sense Multiple Access with Collision Avoidance (CSMA/CA) in IEEE 802.15.4. Further performance and reliability improvement in WBANs can potentially be achieved by combining CSMA/CA with Time Division Multiple Access (TDMA). This essentially requires us to simultaneously design a hierarchical system of FLCs. For this purpose, in Chapter 5, we plan to develop new cooperative coevolution design techniques to further improve the usefulness of WBANs in practice. 


\section{Chapter 5}

\section{Cooperative Design of Two Level Fuzzy Logic Controllers for Medium Access Control in Wireless Body Area Networks}

\subsection{Introduction}

In Chapter 3, we have developed the Cross-Layer Fuzzy logic based Backoff controller (CLFB) to control channel access at the sensor level and improve network reliability and performance in IEEE 802.15.4. Nevertheless, it has been shown that the coordinator level control can also play an important role to improve the reliability and performance of the network [135, 64].

The coordinator in IEEE 802.15.4 manages the GTS mechanism, which can guarantee the reliability and performance of data delivery by allocating a specific time slot within a superframe for data transmissions. However, IEEE 802.15.4 is limited in allocating GTS to the number of nodes as only up to seven GTSs can be allocated during each superframe. This means that many sensor nodes may not enjoy the benefits of accessing a guaranteed service. This limitation might be worsened because of the use of 
the inflexible First-Come, First-Served (FCFS) GTS allocation policy and the waste of the allocated resources. For example, a node with a low data rate that has been allocated a GTS may use it only partially (when the amount of guaranteed bandwidth is higher than its data rate). This leads to underutilisation of the GTS bandwidth resources. In other words, the coordinator, regardless of data rate requirement and traffic congestion, allocates GTS to the requested devices based on FCFS. Therefore, a high data rate sensor may not receive the GTS allocation and a low data rate sensor might underutilise the allocated resources.

To address above mention limitation, some research efforts have been performed to optimised the GTS allocation. For example, Huang et al. [64] proposed an Adaptive GTS Allocation (AGA) method to prioritise GTS allocations in a dynamic fashion based on recent GTS usage feedback with the consideration of low latency and fairness. Zhou et al. [177] proposed Fuzzy Control Medium Access (FCMA) to prioritise GTS allocation based on message criticality and application data rate. However, none of these algorithms has considered either the channel condition while allocating GTS or simultaneously improving both CSMA/CA and GTS through a control strategy to improve reliability and performance of the network.

This chapter proposes a Cooperative Cross Layer Fuzzy Medium Access Control (CoCLF-MAC), which uses FLCs both at the sensor level and the coordinator level to cooperatively improve the quality of the IEEE 802.15.4based MAC in the context of WBANs. Specifically, the coordinator employs FLCs for prioritising GTS allocation by using cross-layer information such as channel condition and application data rate. Meanwhile, sensor nodes utilise CLFB to control channel access.

In Chapter 4, we have shown that careful design of FLCs is essential for successful communications in WBANs. Due to inherent interactions between sensor nodes and the coordinator, FLCs used by sensor nodes and the coordinator must cooperatively work together to achieve desirable results. Consequently, both FLCs need to be designed simultaneously. 
Meanwhile, designing two-level control schemes will significantly increase the complexity of the design process and potentially affect the effectiveness of the designed FLCs. Designing two FLCs jointly will obviously increase the design complexity. To address this issue, following the divideand-conquer strategy, Potter and Jong [126] proposed the CCEA algorithm. In their algorithm, a problem is decomposed into several smaller subcomponents and each sub-component can be evolved through a separate Genetic Algorithm (GA) sub-population. A few years later, Bergh and Engelbrecht [156] proposed Cooperative Particle Swarm Optimiser (CPSO) that can perform much better than the original PSO on several benchmark optimisation problems. This is achieved by using multiple sub-populations to optimise different components of the solution vector cooperatively. In this research, we decide to adopt a standard CPSO because of its proven effectiveness [156] and our familiarity.

In order to use CPSO effectively, one major issue to be addressed in this thesis is on the choice of collaborators from one sub-population while evaluating the candidate FLC design from the other sub-population of CPSO. Typically researchers prefer to choose the best fit solution from alternative sub-populations $[126,156,161]$. However, as a result of using this greedy method, CCEAs and CPSO could fall easily into local optima [156, 161]. Therefore, some researchers have selected an alternative method which involves two candidate solutions, i.e. the best and a random candidate solution $[92,161]$. In this method, both selected collaborators are evaluated with the current candidate solution and the higher fitness determines the fitness of the current candidate solution [156, 161]. Although these methods are commonly used by researchers, they are designed to tackle general optimisation problems. Therefore, in this research, we propose three new collaborator selection schemes based on our insight into efficient communication in WBANs and employ the network knowledge to judge the suitability of any potential collaborators. 


\subsubsection{Chapter Goals}

Most of the existing research works considered only single-level FLCs. In this chapter, we propose a two-level control scheme at both the sensor level and the coordinator level to improve both the reliability and performance of MAC in WBANs. We also propose to use CPSO to automate the design of our two-level control scheme. Following are the chapter goals briefly:

1. Extending IEEE 802.15.4 to facilitate a two-level cross-layer FLC to take advantage of both distributed and centralised mechanisms, i.e at both sensor level and coordinator level, to cooperatively enhance reliability and performance of WBANs.

2. Automatically designing multilevel FLCs for cross-layer MAC involving sensor nodes and the coordinator through CPSO.

3. Introducing new methods of collaborator selection in CPSO by exploiting the specific characteristics of the design problem to improve the algorithm's practical usefulness.

4. Demonstrating the practical usefulness of our CPSO-based multilevel FLC design approach in WBANs by experimentally comparing it with several existing algorithms such as IEEE 802.15.4 [3], AGA [64], FCMA [177], CLFB [115] and $D^{2}$ MAC [107].

\subsubsection{Chapter Organisation}

The remainder of this chapter is organised as follows. Section 5.2 proposes a multilevel cross-layer FLC for prioritising GTS allocations. Section 5.3 presents the CPSO-based FLCs design in WBANs. Section 5.4 reports and analyses the simulation results. Finally, in Section 5.5 the summary will be presented. 


\subsection{A Cross-Layer GTS Allocation Mechanism us- ing Fuzzy Logic}

In this chapter, we aim at proposing CoCLF-MAC which is a two-level control scheme at both the sensor level and the coordinator level, to improve IEEE 802.15.4 in the context of WBANs. As shown in Fig. 5.1, sensor-level FLC is used by sensors to adaptively adjust the backoff exponent, called fuzzyBE, to avoid collisions based on $\mathrm{NB}_{\text {Rate }}$ and the application data rate. Being the moving average of the Number of Backoffs (NB) in IEEE 802.15.4, $\mathrm{NB}_{\text {Rate }}$ serves as a good indication of the channel condition. On the other hand, the application data rate is used to regulate the delay based on the channel access frequency.

At the coordinator-level, Cross-Layer Fuzzy logic GTS Allocation (CLFGA) is utilised to prioritise the requests for transmissions through GTS based on both the channel collision rate and application data rate. Instead of serving each request according to the simple FCFS order [3], the coordinator assigns communication bandwidth to sensor nodes by following their respective fuzzyGTSpriority.

As presented in Algorithm 2, CLFGA adaptively prioritises GTS allocation based on different channel conditions and application requirements. CLFGA utilises a FLC to adaptively assign priorities, so-called fuzzyGTSpriority, to sensor nodes, that are in need of GTS allocations. This assignment is based on recent network conditions experienced by the coordinator and application requirements of sensor nodes.

After prioritising GTS allocation for all sensor nodes, the coordinator adequately allocates GTS resources to sensor nodes. This allocation is based on the priority numbers, GTS capacity and length of the superframe. As presented in Algorithm 2, CLFGA first confirms there are enough GTS resources to be allocated. As we strictly follow IEEE 802.15.4 MAC protocol, CLFGA allocates GTS whenever the following criteria are satisfied: 


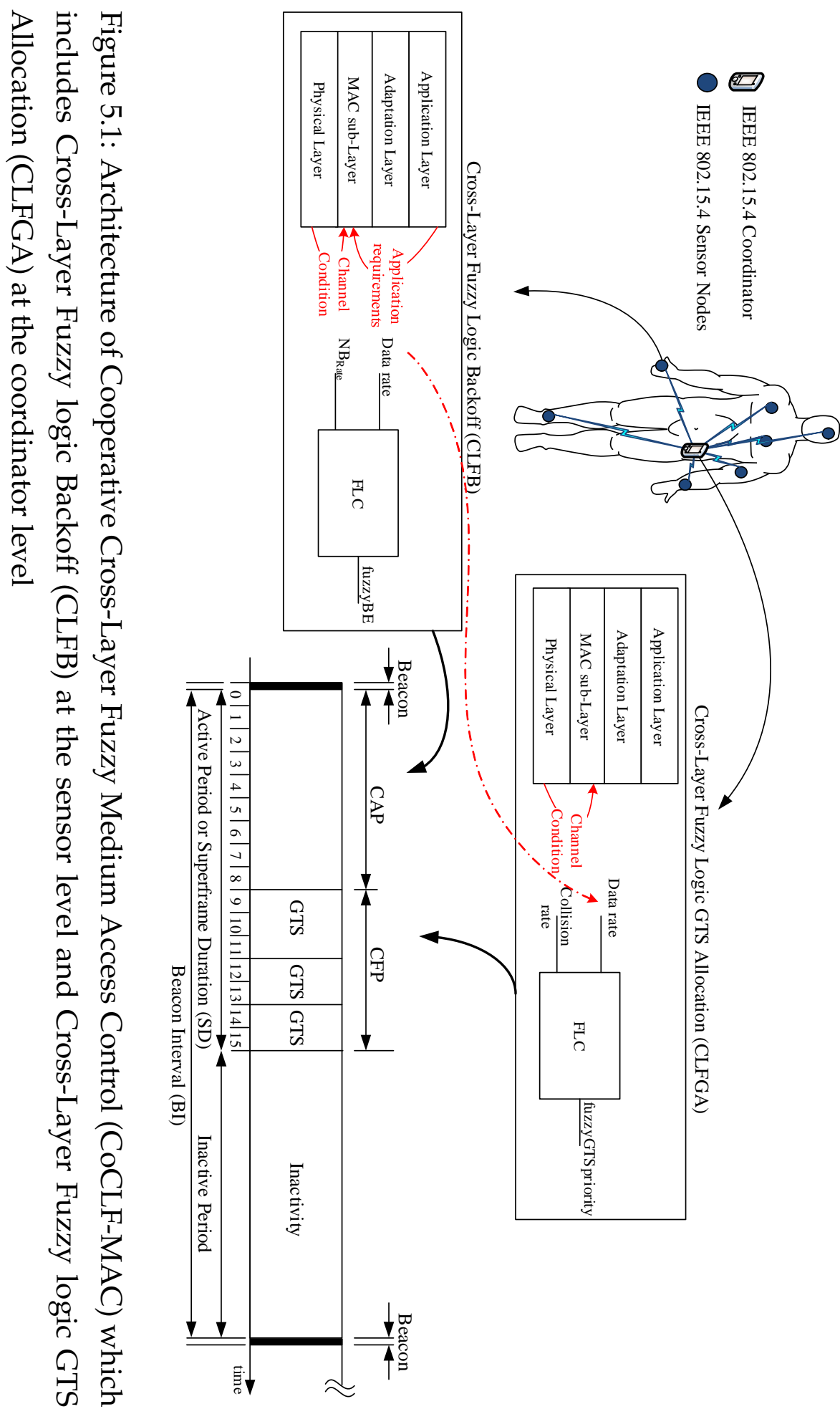


define : Assume that there are $\mathrm{N}$ sensor nodes in a WBAN

Each sensor node sends a GTS allocation request to the coordinator The coordinator allocates GTS to sensor nodes ;

for each sensor node $i \in[1 . . N]$ do

Calculate fuzzyGT Spriority by $_{i}$ the designed FLC based on channel condition and application data rate;

end

while The GTS capacity is not overloaded do

Find sensor node $k$ which has the highest fuzzyGTSpriority;

GTS will be assigned to the sensor node $k$ in the current superframe;

end

Algorithm 2: The pseudocode of Cross-Layer Fuzzy logic GTS Allocation (CLFGA) algorithms.

1. The maximum number of GTS slots to be allocated to devices is seven.

2. The minimum length of a CAP shall be aMinCAPLength. The increase in the total GTS period shall not result in reduction of the CAP length to less than aMinCAPLength.

If both criteria above are satisfied, CLFGA is able to allocate GTS resources to sensor nodes with higher fuzzyGTSpriority (s).

Based on the above discussion, the FLC in CLFGA utilises two input variables, i.e. collision rate and data rate, which are shown in Fig 5.2 and will be described in more detail below. The standard Mamdani fuzzy system will be used for inferencing in our FLC [100].

\subsubsection{Fuzzy Input and Output Variables}

The first input variable of the FLC in CLFGA is collision rate which is measured as follows:

$$
\text { Collision rate }=\frac{\text { Total number of collided signals }}{\text { Total number of recieved signals }}
$$




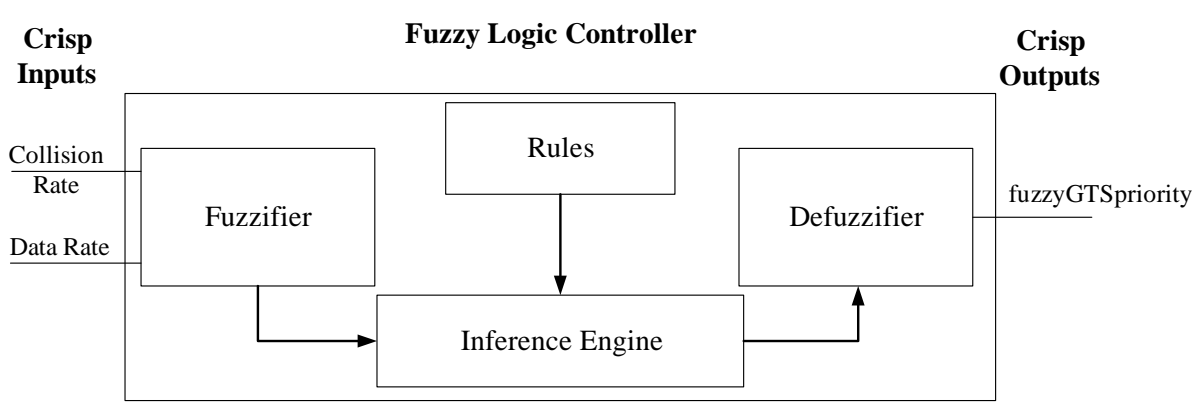

Figure 5.2: Architecture of fuzzy logic controller for CLFGA.

As wireless communication uses a shared medium, if more than one device tries to communicate simultaneously, collisions will happen. If collisions happen, the coordinator will detect the collided signals and abort communication. Therefore, collision rate, which is calculated by the coordinator, is a direct indication of the channel condition in the recent past. Clearly the increase in collision rate indicates high traffic workload in WBANs. Similarly, whenever traffic workload in the network is reduced, collision rate decreases. Collision rate therefore helps to balance the traffic workload between the CFP and CAP. When the CAP traffic load is light, there is no need to allocate too many GTS resources for sensor nodes. Too much dedicated bandwidth for GTS usage leads to resource wastage and to the degradation of the overall system performance. Instead, the GTS bandwidth should be saved for contention-based accesses in CAP. Following Chapter 3, collision rate is fuzzified into four fuzzy levels, namely low, medium, medium-high and high.

The second input variable of our FLC in Fig 5.2 is the data rate of each sensor node. This should be taken into account to adaptively prioritise GTS allocations. For example, nodes with high data rate tend to access the channel more frequently and should be assigned higher priorities. This is important for reducing collisions and packet loss.

In a WBAN, the data rate can vary significantly across different types of nodes. In order to cope with different network settings, the data rate is 
normalised in CLFB within the interval of [1...100]. The normalised data rate is further partitioned into four different fuzzy levels, i.e. low, medium, medium-high and high.

The output from our FLC in Figure 5.2 is the fuzzyGTSpriority. In order to have a reasonable level of accuracy, fuzzyGTSpriority is decomposed into four separate fuzzy levels, which are P1, P2, P3 and P4. Similar to the FLC in CLFB, the centre of gravity is used to defuzzify fuzzyGTSpriority and produce a crisp value.

Moreover, for simplicity and efficiency, similar to CLFB, we use the triangular shaped membership function for all input and output variables. Examples of such membership functions are illustrated in Figure 4.4. In this research, the control parameters of these membership functions, e.g. a, $\mathrm{b}$ and $\mathrm{c}$ in Figure 4.4, are automatically designed by CPSO. In this research, we design membership functions of our FLCs to effectively prioritise GTS allocations and adaptively produce backoff delays. Therefore, GTS and CSMA/CA can consistently work to cooperatively improve network reliability and performance.

\subsubsection{Fuzzy Logic Rules}

In this study, there are 16 different rule antecedents (i.e. 4 fuzzy levels of collision rate $\times 4$ fuzzy levels of data rate). Consequently, our FLC in CLFGA comprises 16 separate fuzzy rules. Each rule follows a basic structure as shown below:

$$
\begin{aligned}
R^{(n)} & : \text { IF Collision - } \text { Rate }_{n} \text { is } a_{1}^{i} \\
& \text { and Data - Rate } \text { is }_{2}^{i} \\
& \text { THEN fuzzyGTSPriority } \text { TS }_{n}{ }^{i}
\end{aligned}
$$

A summary of all fuzzy rules in CLFGA is presented in Table 5.1. According to this table, whenever collision rate is low, our FLC will consistently suggest the lowest priority $P 1$, e.g. fuzzy rules $R^{(1)}, R^{(2)}, R^{(3)}$ and $\mathrm{R}^{(4)}$ in Table 5.1. This helps to prevent deteriorating network performance 
by not allocating too many GTS resources to sensor nodes. In the case that collision rate is at a Medium level, our FLC will recommend moderate fuzzyGTSpriority, e.g. $\mathrm{R}^{(6)}$ and $\mathrm{R}^{(7)}$. Meanwhile nodes with high data rates will receive higher priority, e.g. $\mathrm{R}^{(8)}$. The node with a Low data rate still receives lowest priority to prevent wasting GTS resources, e.g. $\mathrm{R}^{(5)}$. Furthermore, if the channel becomes Medium-High, the priorities of corresponding rules, i.e. $\mathrm{R}^{(10)}$ and $\mathrm{R}^{(11)}$, will be adjusted to $\mathrm{P} 3$. For the same reason, the nodes with Low data rate receive lower priority, i.e. $\mathrm{R}^{(9)}$, and the node with a High data-rate will have the highest priority, i.e. P4. Finally when traffic workload is High, our FLC will produce the highest priority P4 for $\mathrm{R}^{(15)}$ and $\mathbf{R}^{(16)}$. The nodes with Low and Medium data rate will still receive relatively lower priority, i.e. P3.

Table 5.1: Fuzzy Logic rules for CLFGA.

\begin{tabular}{|l|c|c|c|c|}
\hline \multirow{2}{*}{ Collision Rate } & \multicolumn{4}{|c|}{ Data Rate } \\
\cline { 2 - 5 } & Low & Medium & Medium High & High \\
\hline Low & $R^{(1)}: P 1$ & $R^{(2)}: P 1$ & $R^{(3)}: P 1$ & $R^{(4)}: P 1$ \\
\hline Medium & $R^{(5)}: P 1$ & $R^{(6)}: P 2$ & $R^{(7)}: P 2$ & $R^{(8)}: P 3$ \\
\hline Medium High & $R^{(9)}: P 2$ & $R^{(10)}: P 3$ & $R^{(11)}: P 3$ & $R^{(12)}: P 4$ \\
\hline High & $R^{(13)}: P 3$ & $R^{(14)}: P 3$ & $R^{(15)}: P 4$ & $R^{(16)}: P 4$ \\
\hline
\end{tabular}

\subsection{Evolutionary Design of Multilevel FLCs in WBANs}

In this section, we propose a general method to automatically design FLCs for our two-level control scheme as shown in Fig. 5.1. As reported extensively in the literature [159, 45, 59, 11,47], evolutionary computation techniques have been successfully applied to automate the design of various aspects of FLCs and are considered highly suitable for tackling our 
research problems. Specifically, we focus on designing the control parameters of all MFs through CPSO.

In this research, we take advantage of the modularity nature of our design problem. Particularly, we use two sub-populations in CPSO: the first sub-population contains individuals that encode FLCs in CLFB to be used at the sensor level, and the second sub-population contains individuals that encode the FLCs in CLFGA to be used at the coordinator level.

In the context of WBANs, we can identify four important technical issues for CPSO-based design of FLCs:

1. Coding scheme: Each candidate solution in CPSO represents a separate FLC design and must follow a specific coding scheme which encodes all control parameters for each MF through a vector of real numbers. In Chapter 4, we have studied the influence of different coding schemes on the effectiveness and interpretability of the FLCs designed. We experimentally showed that Moderately Restricted Coding Scheme (MRCS), as illustrated in Fig. 4.4, can provide both good effectiveness and interpretability. Consequently, in this chapter, we utilise MRCS to encode candidate FLCs at both control levels.

2. Fitness function: Fitness function is an essential part of our design approach. Through properly designed fitness functions, we can obtain a desirable balance between reliability and performance in WBANs. Our main design goal is to optimise the reliability of WBANs (measured by PDR). On the other hand, the performance of the network has to remain competitive with respect to IEEE 802.15.4. In WBANs, throughput can be improved directly through increasing PDR. However, increasing PDR may lead to an increase in packet delay. We therefore decide to pursue PDR as the main objective and packet delay as a soft constraint, the violation of which will be penalised through the fitness function. Consequently, we have defined the 
fitness function as follows:

$$
F=\Delta P D R+\lambda \times \Delta \text { Delay }
$$

where $\lambda$ is a coefficient. In chapter 4 , we have studied the particular effect of $\lambda$ and found that a proper value for $\lambda$ to obtain a desirable trade-off between PDR and delay is 2.5.

3. Collaborator selection: In CPSO, as each candidate FLC is part of the whole solution, while evaluating the fitness of any candidate FLC from one sub-population, we need to select a candidate FLC from the other sub-population as a collaborator. In this research, we will try to exploit our network knowledge to select collaborators more effectively (refer to Subsection 5.3.2).

4. Evaluation method: In order to ensure the practicality of our approach, we have introduced two different evaluation targets. The first target is designing effective FLCs to provide reliability and performance for a specific WBAN configuration. The second target aims to design desirable FLCs for more general WBAN settings.

\subsubsection{Multilevel FLC Design based on CPSO}

CPSO [156], which is explained in Algorithm 3, decomposes candidate FLCs into two sub-populations of s-dimensions, one for CLFB and one for CLFGA. In order to evaluate the fitness value of each candidate FLC of corresponding sub-population, CPSO builds a collaborator vector $\tilde{y}$. As shown in Figure 5.3, $\tilde{y}$ is built by selecting candidate FLCs from corresponding sub-population as collaborators. Function $b\left(j, P_{j} . x_{i}\right)$ is called to evaluate the $i$ th candidate FLC in the $j$ th sub-population. This function returns an $n$-dimensional vector as the whole solution containing $\tilde{y}$ with its $j$ th component replaced by $P_{j} . x_{i}$. The idea is to evaluate how well $P_{j} . x_{i}$ "cooperates" with another sub-population. 
define $: b(j, z) \equiv\left(P_{1} \cdot \tilde{\mathrm{y}}, \ldots, P_{j-1} \cdot \tilde{\mathrm{y}}, z, P_{j+1} \cdot \tilde{\mathrm{y}}, \ldots, P_{K} \cdot \tilde{\mathrm{y}}\right)$

Create and initialise $(K=2)$ sub-populations, i.e. candidate FLCs for CLFB and CLFGA, each with $s$ dimensions (where $n=K \times s$ );

The $j$ th sub-population is denoted as $P_{j}, j \in[1 . .2]$;

repeat

for each sub-popualtion $P_{j} \in[1 . . K]$ do

for each candidate FLC $i \in[1 . . s]$ do

if $f\left(b\left(j, P_{j} \cdot x_{i}\right)\right)<f\left(b\left(j, P_{j} \cdot\right.\right.$ pbest $\left.\left._{i}\right)\right)$ then

$P_{j}$.pbest $t_{i} \leftarrow P_{j} . x_{i} ;$

end

if $f\left(b\left(j, P_{j} . p b e s t_{j}\right)\right)<f\left(b\left(j, P_{j} . g b e s t\right)\right)$ then $P_{j}$.gbest $\leftarrow P_{j}$. pbest ${ }_{j} ;$

end

end

Perform PSO updates, i.e. velocity and position, on $P_{j}$;

end

until termination condition is true;

Algorithm 3: The pseudocode of the CPSO algorithm for designing our multilevel FLCs. $P_{j} . x_{i}$ denotes the current position of the $i$ th candidate FLC of the $j$ th sub-population. $P_{j}$.pbest $t_{i}$ is the personal best of the $i$ th candidate FLC of the $j$ th sub-population. The $j$ th of the $K$ sub-population has a global best particle $P_{j}$.gbest. 
The original CPSO chooses the best individuals from other sub-populations as collaborators. However, this approach is greedy and might fall in local optima [161]. Therefore, in the following subsection, we study different approaches to choose collaborators to cooperate with the other sub-population (or sub-populations) for improved design performance.

\subsubsection{Collaborator Selection Method}

A candidate solution in CPSO can not be evaluated from a single population in isolation, because each candidate solution represents only a part of the whole solution. Therefore, collaborators must be chosen from other sub-populations to assess fitness. Particularly, in each generation, CPSO evaluates each candidate solution of every sub-population by combining them with collaborators from other sub-populations. Similarly, CPSO follows the procedure for all the remaining sub-populations as shown in Fig 5.3.

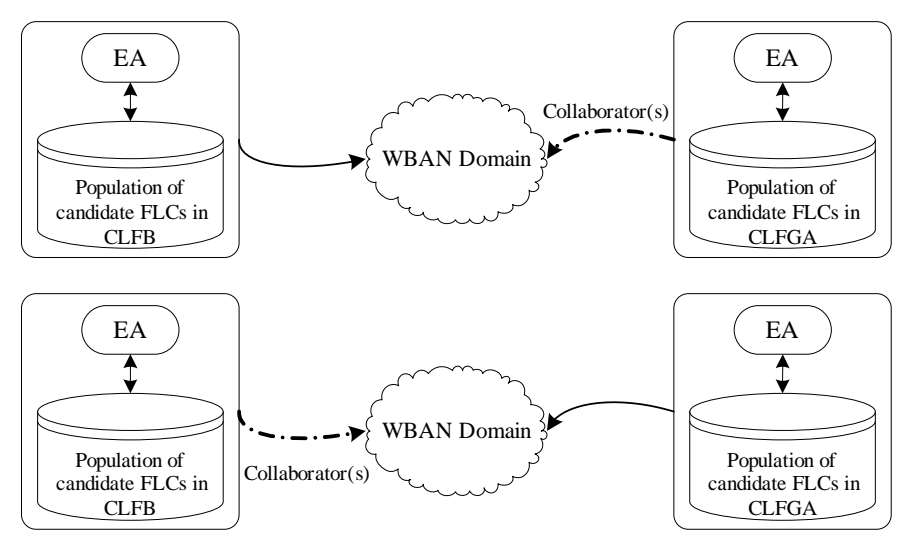

Figure 5.3: Coevolutionary model of two populations

There are two straightforward ways to select collaborators from other sub-populations: (1) select the fittest candidate solution or gBest; and (2) select a random candidate solution. The former is greedy and the latter is explorative [161]. Although these methods are commonly used $[161,126]$, they are designed to tackle general optimisation problems. It 
is interesting to exploit the unique characteristic of our design problem to find more suitable collaborators. In following, we will explain two alternative approaches, which use expert knowledge to find more effective collaborators.

\section{Similarity of shared input variable}

In our design problem, we suggest using possible correlations between two FLCs, which can be useful to find a potential collaborator. As shown in Fig 5.1, data rate is the common input variable for the both FLCs. Moreover, rules, which are designed for FLCs in CLFB and CLFGA, treat data rate almost in the same manner. Particularly, in CLFB, whenever data rate increases from low to high, FLC suggests increasing backoff delays accordingly. Similarly, in CLFGA, when data rate increases from low to high, FLC also suggests increasing the priority of GTS allocation to the corresponding sensor node. Therefore, we suggest exploiting the similarity of data rate MFs to find more suitable collaborators. Specifically, CPSO calculates similarities between the current candidate solution and candidate solutions from the alternative sub-population and assigns a similarity degree to each potential collaborator. Afterwards, CPSO selects a more similar candidate FLC from the the other sub-population as a collaborator. To calculate the similarity degree, as illustrated in Fig 5.4, we first measure the Euclidean distances between control points in each MF as follows:

$$
\left\{\begin{array}{l}
m_{i j}=\sqrt{\left(a_{i j}-b_{i j}\right)^{2}}=\left\|a_{i j}-b_{i j}\right\|, \quad \text { where } i=1,2 \\
n_{i j}=\sqrt{\left(c_{i j}-b_{i j}\right)^{2}}=\left\|c_{i j}-b_{i j}\right\|, \quad \text { and } j=1,2,3,4
\end{array}\right.
$$

where $i$ is the candidate solution number and $j$ is the number of MFs.

Afterwards, the similarities between each MF in the current candidate solution and its corresponding MF in the potential collaborator are measured by Membership Function Similarity Degree (MFSD) as below:

$$
\text { MFSD }=\sqrt{\left(x_{k j}-x_{h j}\right)^{2}+\left(m_{k j}-m_{h j}\right)^{2}+\left(n_{k j}-n_{h j}\right)^{2}} \text {, where } k \neq h
$$



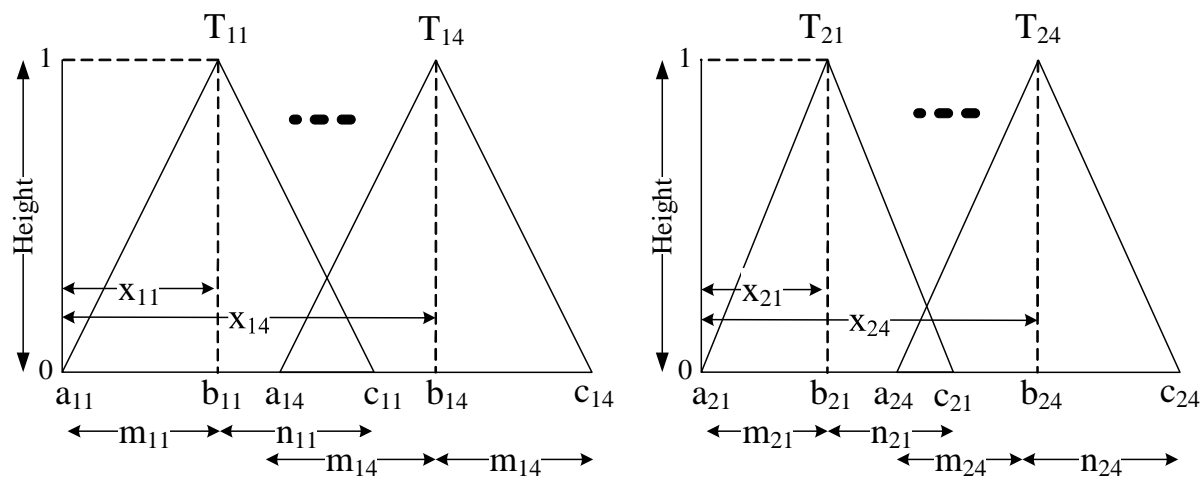

Figure 5.4: An example of similarity measurements

where $k$ is MF in the current candidate solution and $h$ is the corresponding MF of the potential collaborator. Finally, the Similarity Degree (SD) between the current candidate solution and the potential collaborator from another sub-population is measured as follows:

$$
S D=\sqrt{\frac{1}{j} \sum_{i=1}^{j}\left(M F S D_{i}-\overline{M F S D}\right)^{2}} \text {, where } \overline{M F S D}=\frac{\sum_{i=1}^{j} M F S D_{i}}{j}
$$

where SD suggests that two MFs are more similar to each other when their $\mathrm{SD}$ is smaller.

\section{Network inspired Collaborator Selection (NiCS)}

The previous method of selecting a collaborator was based on similarity of shared rules between two FLCs. However, this decision might become more precise with help of the deeper knowledge of experts. Therefore, we introduce Network inspired Collaborator Selection (NiCS) to select collaborators more effectively. Based on our experience of effective communication in WBANs, the criteria for collaborator selection have been summarised in Table 5.2 and 5.3. These criteria define the support of MFs for both sensor-level and coordinator-level FLCs. The basic idea is to ensure that 
Table 5.2: Collaborator selection criteria for sensor-level FLC design

\begin{tabular}{|l|c|c|c|c|}
\hline \multirow{2}{*}{$\begin{array}{l}\text { Input and } \\
\text { output variable }\end{array}$} & \multicolumn{4}{|c|}{ Support of membership function } \\
\cline { 2 - 5 } & Low/B1 & Medium/B2 & Medium High/B3 & High/B4 \\
\hline $\mathrm{NB}_{\text {Rate }}$ & {$[0 \ldots 1)$} & {$[0 \ldots 3]$} & {$[1 \ldots . .5]$} & {$[4 \ldots 6]$} \\
\hline Data rate & {$[1 \ldots 50]$} & {$[10 \ldots 75]$} & {$[35 \ldots 100)$} & {$[50 \ldots 100]$} \\
\hline fuzzyBE & {$[2 \ldots . .5]$} & {$[3 \ldots 6]$} & {$[5 \ldots .7 .5]$} & {$[6 \ldots 8]$} \\
\hline
\end{tabular}

Table 5.3: Collaborator selection criteria for coordinator-level FLC design

\begin{tabular}{|l|c|c|c|c|}
\hline \multirow{2}{*}{$\begin{array}{l}\text { Input and } \\
\text { output variable }\end{array}$} & \multicolumn{4}{|c|}{ Support of membership function } \\
\cline { 2 - 5 } & Low/P1 & Medium/P2 & Medium Hig/P3 & High/P4 \\
\hline Collision rate & {$[0 \ldots 30]$} & $(0 \ldots 80]$ & {$[40 \ldots 100)$} & {$[70 \ldots 100]$} \\
\hline Data rate & {$[1 \ldots 40]$} & {$[10 \ldots 60]$} & {$[35 \ldots 90]$} & {$[50 \ldots 100]$} \\
\hline fuzzyGTSpriority & {$[0 \ldots 30]$} & $(0 \ldots 70]$ & {$[30 \ldots 100)$} & {$[80 \ldots 100]$} \\
\hline
\end{tabular}

the support of partnering FLCs always falls into the right range which may help to promote a more effective collaboration.

According to Table 5.2, while CPSO is evolving FLCs at the coordinator level, we need to carefully select a FLC collaborator at the sensor level. For example, in view of rules $\mathrm{R}^{(1)}, \mathrm{R}^{(2)}$ and $\mathrm{R}^{(3)}$ in Table 3.1, the support of B1 MF for fuzzyBE is set to [2..5] in Table 5.2. Consequently, sensor nodes do not need to wait before packet transmission as long as the channel is not busy. The rules in Table 3.1 also suggest that, when the channel is highly congested, sensor nodes must apply backoff delay long enough to prevent collisions. Consequently, the support of $\mathrm{B} 4 \mathrm{MF}$ is in the range of [6..8].

As an another example, the support of Low MF for $N B_{\text {rate }}$ in Table 5.2 is in the range of [0..1). This means that the channel is mostly free and highly likely a sensor node can send its packet within the current Contention Window (CW). On the other hand, High MF shows an extremely busy channel and its support falls in [4...6].

Similarly, selection criteria in Table 5.3 guide the selection of effective 
collaborators at the coordinator level to evolve FLCs in sensor level. For example, in view of the rules $R^{(1)}, R^{(2)}$ and $R^{(3)}$ in Table 5.1, the support of P1 MF shown in Table 5.3 implies that, as long as the channel is not busy, the coordinator does not need to assign communication bandwidth to the requested sensor nodes immediately. On the other hand, based on the rules $\mathrm{R}^{(12)}, \mathrm{R}^{(15)}$ and $\mathrm{R}^{(16)}$ in Table 5.1, the support of P4 MF suggests that the coordinator needs to assign the channel to the requested sensor nodes immediately to prevent collisions when the channel is highly congested. As another example, the support of Low MF for collision rate falls in [0..30], implying that the coordinator believes that the channel is fairly clear and will therefore assign the lowest priority to any requested sensor nodes.

As explained in Algorithm 4, if a candidate FLC meets these criteria, it could be considered as a potential collaborator. Otherwise, the collaborator can be chosen either randomly or as the fittest FLC candidate (gBest). In our new approach, we can select the candidate FLC from the pool of potential collaborators (NiCSpool) in two ways:

1. $\mathrm{NiCS_{gBest }}$ : if the fittest solution is within the potential collaborators, it is selected as the collaborator to evaluate the candidate solution; otherwise, the collaborator will be randomly selected from NiCSpool.

2. $\mathrm{NiCS}_{\text {random }}$ : randomly select a collaborator from the potential collaborators, regardless of whether the fittest selection is within NiCSpool.

In the literature, most of the research works have only studied general collaborator selection methods [161]. However, it is not clear which collaborator selection method is particularly useful for our design problem. Therefore, in this research, in order to discover an effective collaborator selection method, five collaborator selection methods will jointly be studied in the context of WBANs (refer to Section 5.4.) 
define : NiCSpool is a pool of potential collaborators for each sub-population, i.e. candidate FLCs for CLFB and CLFGA;

The $j$ th sub-population is denoted as $P_{j}, j \in[1 . .2]$;

for each sub-population $j \in[1 . .2]$ do

Copy potential collaborators into $\mathrm{NiCSpool}_{j}$ based on criteria in

Tables 5.2 and 5.3;

if $\mathrm{NiCSpool}_{j}>0$ then

if $\mathrm{NiCSgBest==enable} \mathrm{and} P_{j}$. gbest $\in N i C$ Spool $_{j}$ then

$P_{j} \cdot \tilde{\mathrm{y}}=P_{j}$. gbest;

else

| randomly choose $P_{j} \cdot \tilde{y}$ from $\mathrm{NiCSpool}_{j}$;

end

else

if $\mathrm{NiCSgBest==enable}$ then

$P_{j} . \tilde{\mathrm{y}}=P_{j}$. gbest;

else

| randomly choose $P_{j} . \tilde{y}$ from sub-population $j t h$;

end

end

end

Algorithm 4: The pseudocode of the NiCS algorithm. $P_{j} . \tilde{y}$ denotes the selected collaborator in the $j$ th sub-population. The $j$ th sub-population has a global best particle $P_{j}$. gbest 


\subsubsection{Fitness Evaluation Method}

In this research, two methods have been studied to evaluate the fitness of each candidate solution guided by two different design targets [115]. The first design target $\left(\mathrm{T}_{1}\right)$ is to design FLCs that function effectively on a specific network setting. The second target $\left(\mathrm{T}_{2}\right)$ is to design FLCs that are able to work consistently well across a wide range of network settings. To distinguish design targets, every result upon using our CoCLF-MAC based on targets $T_{1}$ and $T_{2}$ will be referred to as CoCLF-MAC $T_{T_{1}}$ and CoCLF$\mathrm{MAC}_{T_{2}}$ respectively.

Consequently, we consider two different evaluation methods to support these two design targets. The first method evaluates the candidate solution based on a single pre-defined WBAN setting. The second evaluation method utilises multiple pre-determined WBAN settings to jointly evaluate the same candidate solution. In the second approach, to determine the fitness of the candidate solution, the averaged $\triangle P D R$ and $\triangle$ Delay over multiple network settings will be utilised [115]. In both evaluation methods, every network setting will be tested for five independent runs. For detailed information, please refer to Subsection 5.4.5.

In this research we are not employing any overfitting control techniques while designing FLCs. However, we have experimentally demonstrated the effectiveness of designing FLCs through CPSO-based approach to improve the reliability and performance of varied WBAN's settings. In the future work, we will take advantage of using overfitting control techniques [35].

\subsection{Simulation Implementation and Results}

In this section, we describe the simulation settings for WBANs in Subsection 5.4.1, the CPSO parameters settings in Subsection 5.4.2, and the evaluation metrics in Subsection 5.4.3. Afterwards, the simulation results will be reported in two subsections. Particularly, in Subsection 5.4.4, the re- 
sults will be presented for designing our multilevel FLC to achieve target $T_{1}$, i.e. multilevel FLCs for specific network settings. We will particularly investigate the effectiveness of different collaborator selections. Subsection 5.4.5 analyses the simulation results obtained from designing our multilevel FLC with target $T_{2}$, i.e. multilevel FLCs for multiple network settings. Through comprehensive simulation studies, we can finally conclude in this section that our EC-based approach for multilevel FLC design can outperform many competing algorithms in the literature including, IEEE 802.15.4 [3], CLFB [115], D²MAC [107], AGA+ IEEE 802.15.4 [64], AGA+CLFB [64, 115] and FCMA [177].

\subsubsection{Simulation Environment for WBAN}

In order to simulate WBAN, we have utilised OMNeT++ network simulator version 4.4.1 [5]. Following typical settings in the literature [119], the maximum data rate is $250 \mathrm{~Kb} / \mathrm{s}$ with frequency of $2.4 \mathrm{GHz}$. In our simulation, all sensor nodes are randomly deployed within a $2 \times 2 \mathrm{~m}^{2}$ space with a single WBAN coordinator using the star topology. The network simulation time for our experiments is 600 seconds (longer simulation time does not appear to alter our major findings). We have used the log-normal shadowing model as the channel model. In comparison with the traditional Rayleigh and Ricean distributions, the log-normal shadowing model reproduces the small-scale fading in WBANs more accurately [147].

\subsubsection{Parameter Settings for Evolutionary Algorithms}

To design two-level FLCs, the size of each sub-population in CPSO is set to 50. The maximum number of generations is set to 100 . Under these settings, CPSO is close to convergence after 50 generations. Meanwhile, the fully connected topology is used for CPSO and $\omega=0.7298$ and $c_{1}=c_{2}=1.49618$ [167]. To have reliable results, CPSO under the same settings is repeated 30 times with different starting seeds. 


\subsubsection{Evaluation Metrics}

To quantitatively compare the reliability and performance of WBANs, we employ four well-known metrics which are PDR, collision rate, throughput and Packet delay.

For a fair comparison with competing algorithms, each competing technology is run for 30 times independently. Again, the average results in reliability and performance is used to compare with FLCs designed by our CPSO-based approach.

In addition, the Analysis of Variance (ANOVA) test is performed to determine whether statistically significant differences in performance and reliability can be observed. Tukey's post-hoc analysis and t-test analysis are also utilised to realise the main source of difference.

\subsubsection{Automatic Design of Multilevel FLCs for Specific Net- work Settings (Target $T_{1}$ )}

This subsection introduces a WBAN simulation scenario for target $\mathrm{T}_{1}$. Afterwards, the effectiveness of five different collaborator selections in CPSO for FLC designs is statistically investigated. Finally the reliability and performance of CLFB and CLFGA achieved by FLCs designed in this research are compared with the competing algorithms.

\section{Network Simulation Scenario for Target $T_{1}$}

Similar to Chapter 4, we consider a specific WBAN scenario where an elderly individual lives alone. She has recently had a procedure. A collection of wireless sensors are attached to her body to monitor her health status. All sensory information is collected and sent to her doctor by a WBAN through the Internet. This WBAN scenario contains three-lead ECG, EEG, respiratory rate and motion sensors as well as a smartphone coordinator. The communication features of these sensor nodes can be found in Table 


\section{4 .}

Table 5.4: Communication specifications of Sensor nodes used in the simulation $[2,119]$.

\begin{tabular}{|l|c|c|c|c|}
\hline Sensor Node & ECG & Respiratory Rate & Motion Sensor & Heart Rate (HR) \\
\hline $\begin{array}{l}\text { Traffic } \\
\text { Generation } \\
\text { Distribution }\end{array}$ & Constant & Constant & Poisson & Constant \\
\hline Data Rate & $156.25 \mathrm{Bps}$ & $15 \mathrm{Bps}$ & $64 \mathrm{Bps}$ & $20 \mathrm{Bps}$ \\
\hline \hline Sensor Node & Temperature & EEG & Blood Pressure (BP) & Endoscope Imaging \\
\hline $\begin{array}{l}\text { Traffic } \\
\text { Generation } \\
\text { Distribution }\end{array}$ & Poisson & Constant & Constant & Poisson \\
\hline Data Rate & $12.5 \mathrm{Bps}$ & $31.25 \mathrm{Bps}$ & $512 \mathrm{Bps}$ & $1538.46 \mathrm{Bps}$ \\
\hline
\end{tabular}

\section{Collaborator selection}

In this research, we explore five different collaborator selection methods, i.e.

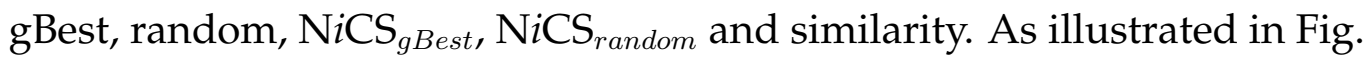
5.5 , the results show that CPSO achieved consistently good results after 100 generations (5000 evaluations) with respect to all collaborator selection methods. Moreover, Fig. 5.5 shows that greedy methods for collaborator selection can be more effective than less greedy selection methods. For example, the gBest collaborator selection method is more effective than the random one. Furthermore, the simulation results confirm that our selection methods, i.e. $\mathrm{NiCS}_{\text {gBest }}$ and $\mathrm{NiCS}$ random, can enhance the effectiveness of the collaborator selection. For example, the $\mathrm{NiCS}_{g B e s t}$ collaborator selection method is more effective than gBest during the first 10 generations in terms of both PDR and packet delay, statistically confirmed by ANOVA and Tukey's post-hoc analysis (p-value $<0.05)$. Similarly, $\mathrm{NiCS}$ random is also more effective than the random collaborator selection method in our design problem. We have also introduced another greedy collaborator selection method based on similarity of shared input variables between two FLCs, 
i.e. CLFB and CLFGA. Although this selection method is more effective than random collaborator selection, it cannot outperform $\mathrm{NiCS}_{\text {random. }}$. Generally, since CPSO with $\mathrm{NiCS}_{g B e s t}$ achieved reasonably good results after 10 generations, whenever there is lack of computation power and time, $\mathrm{NiCS}_{g B e s t}$ is more desirable than other selection methods.

\section{Comparison between CPSO-based FLC Design and Competing Algo- rithms}

In order to demonstrate the effectiveness of CoCLF-MAC $T_{1}$, we have compared it with several cutting-edge algorithms, i.e. IEEE 802.15.4 [3], CLFB [115], D²MAC [107], AGA+ IEEE 802.15.4 [64], AGA+CLFB [64, 115] and FCMA [177]. Among these algorithms, CLFB and $D^{2}$ MAC use one-level control and the rest, i.e. IEEE 802.15.4, AGA+ IEEE 802.15.4, AGA+CLFB and FCMA, use two-level control. As presented in Table 5.5, CoCLF$\mathrm{MAC}_{T_{1}}$ significantly outperforms IEEE 802.15.4, CLFB, D²MAC, AGA+ IEEE 802.15.4, AGA+CLFB and FCMA in terms of both PDR and packet delay. The one-way ANOVAs give significant differences with $\mathrm{p}$-value $<0.0001$. The corresponding Tukey's post-hoc analysis further confirms our conclusion. Specifically, while PDR achieved by CoCLF-MAC $T_{T_{1}}$ is significantly higher than IEEE 802.15.4, CLFB, D²MAC, AGA+ IEEE 802.15.4, $\mathrm{AGA}+\mathrm{CLFB}$ and FCMA, packet delay achieved by CoCLF-MAC $T_{T_{1}}$ is also significantly lower than the rest of the algorithms.

Table 5.5 further reports the WBAN throughput upon using each algorithm. Statistical analysis confirms once again that CoCLF-MAC $T_{1}$ achieves significantly higher throughput than the rest of the algorithms. Moreover, it can also significantly reduce the collision rate in comparison to all competing algorithms. 


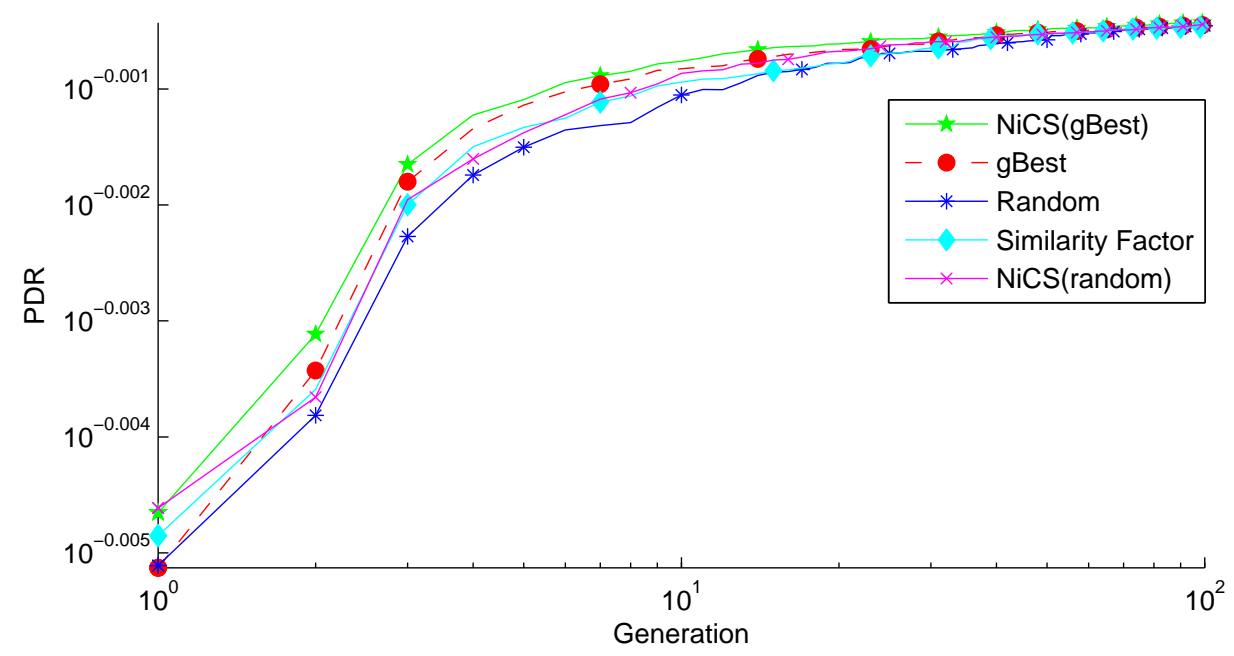

(a) PDR

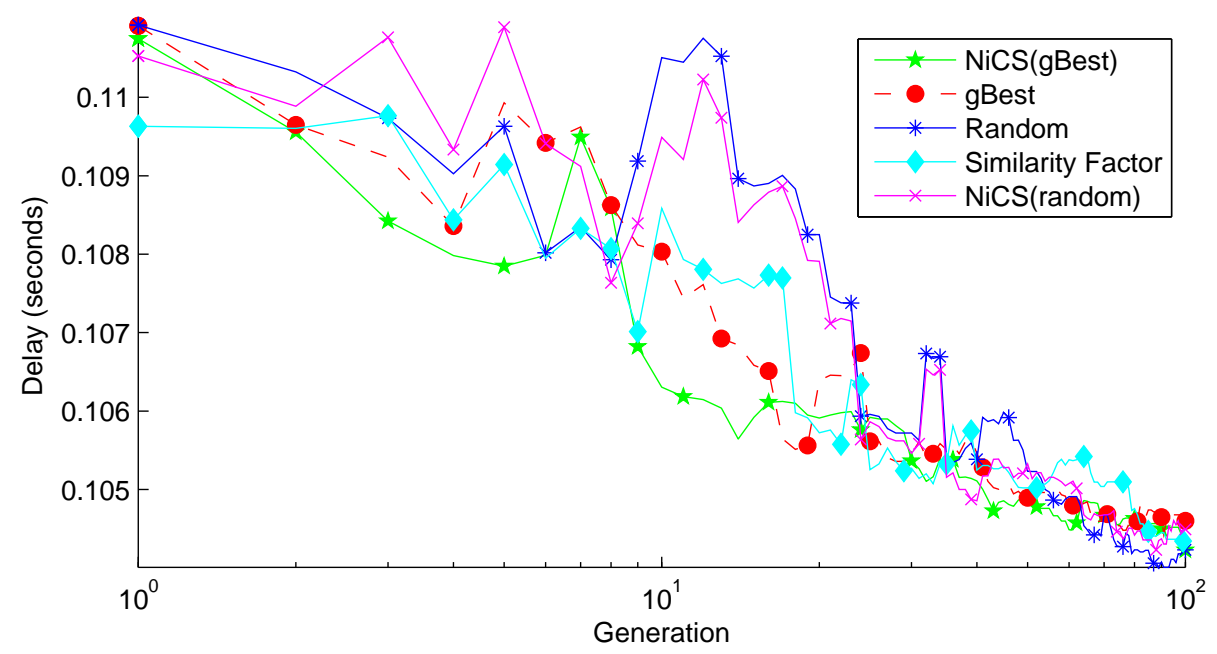

(b) Delay

Figure 5.5: The CPSO-based process for FLC design after 100 generations. The results for all collaborator selection methods are obtained by averaging over 30 independent tests in a four-sensor-node scenario. 
Table 5.5: Comparison of CoCLF-MAC $T_{1}$ and competing algorithms, in terms of PDR and packet delay (seconds), throughput (bps) and Collision rate.

\begin{tabular}{|l|l|l|l|c|}
\hline & PDR & Delay & Throughput & Collision rate \\
\hline IEEE 802.15.4 [3] & $0.831 \pm 0.005$ & $0.168 \pm 0.007$ & $09594.17 \pm 32.93$ & $0.322 \pm 0.014$ \\
\hline D $^{2}$ MAC [107] & $0.845 \pm 0.004$ & $0.271 \pm 0.015$ & $09601.40 \pm 34.75$ & $0.310 \pm 0.014$ \\
\hline CLFB [115] & $0.828 \pm 0.002$ & $0.229 \pm 0.003$ & $09570.21 \pm 16.03$ & $0.331 \pm 0.006$ \\
\hline $\begin{array}{l}\text { AGA [64] } \\
+ \text { IEEE 802.15.4 }\end{array}$ & $0.903 \pm 0.005$ & $0.162 \pm 0.121$ & $10742.47 \pm 72.76$ & $0.162 \pm 0.011$ \\
\hline $\begin{array}{l}\text { AGA[64] } \\
+ \text { CLFB[115] }\end{array}$ & $0.941 \pm 0.002$ & $0.149 \pm 0.002$ & $11061.79 \pm 56.47$ & $0.138 \pm 0.007$ \\
\hline FCMA [177] & $0.924 \pm 4.3 \times 10^{-4}$ & $0.151 \pm 0.001$ & $10906.71 \pm 34.27$ & $0.141 \pm 0.003$ \\
\hline CoCLF-MAC $_{T_{1}}$ & $\mathbf{0 . 9 9 9} \pm \mathbf{2 . 4} \times \mathbf{1 0}^{-5}$ & $\mathbf{0 . 1 0 4} \pm \mathbf{0 . 0 0 1}$ & $\mathbf{1 1 9 7 6 . 0 3} \pm \mathbf{4 9 . 2 0}$ & $\mathbf{0 . 0 0 8} \pm \mathbf{0 . 0 0 5}$ \\
\hline
\end{tabular}

\subsubsection{Automatic Design of Multilevel FLCs for Multiple Network Settings (Target $\mathrm{T}_{2}$ )}

In this subsection WBAN simulation settings for design target $\mathrm{T}_{2}$ will be explained. Accordingly, we statistically study the effectiveness of different collaborator selection methods. Finally, the reliability and performance of CoCLF-MAC $T_{T_{2}}$ are compared with the competing algorithms.

\section{Network Simulation Scenario for Target $\mathrm{T}_{2}$}

Since multilevel FLC designs for target $\mathrm{T}_{1}$ may not produce a desirable balance between reliability and performance over a wide range of WBAN settings, we also aim to design multilevel FLCs based on target $T_{2}$ (i.e. enhancing reliability and performance over multiple WBAN's settings). For this purpose, we have built separate training and testing scenarios. Similar to Chapter 4, five different network settings described in Table 5.6 are used for training. Furthermore, Table 5.7 summarises all testing scenarios for this study. Specifically, to test the generalisability of multilevel FLC designs, we have considered up to eight different sensors during testing. All sensors are 
Table 5.6: WBAN settings for designing Multilevel FLCs regarding target $\mathrm{T}_{2}$.

\begin{tabular}{|c|l|l|}
\hline Scenario & No of sensors & WBAN setting \\
\hline 1 & Three sensors & Temperature, ECG, respiratory rate \\
\hline 2 & Four sensors & Temperature, ECG, respiratory rate and HR \\
\hline 3 & Six sensors & Temperature, ECG, EEG, respiratory rate, HR, and BP \\
\hline 4 & Seven sensors & $\begin{array}{l}\text { Temperature, ECG, EEG, respiratory rate, HR, BP and } \\
\text { Motion }\end{array}$ \\
\hline 5 & Eight sensors & $\begin{array}{l}\text { Temperature, ECG, EEG, respiratory rate, HR, BP, Motion } \\
\text { and Endoscope Imaging }\end{array}$ \\
\hline
\end{tabular}

commonly studied in the literature $[2,119]$. Meanwhile, two ECG sensors are included in testing scenario 9 in Table 5.7 in order to examine WBAN with high traffic load.

\section{CPSO-based FLC Design}

In this section, the training results from CPSO through the five different collaborator selection methods, i.e. gBest, random, $\mathrm{NiCS}_{\text {gBest }}, \mathrm{NiCS}_{\text {random }}$ and similarity, are presented in Table 5.8. The results in Table 5.8 show that CPSO can be more effective through greedy methods in our design problem. For example, the gBest collaborator selection method is more effective than the random one. From the results, we found that $\mathrm{NiCS}_{g B e s t}$ generally outperforms other collaborator selection methods. Specifically, the results clearly show that, except scenarios one and two, CPSO through $\mathrm{NiCS}_{g \text { Best }}$ is more effective than other collaborator selection methods, statistically confirmed by ANOVA and Tukey's post-hoc analysis (p-value $<0.05)$. Furthermore, the design process through our selection method, i.e. $\mathrm{NiCS}_{\text {random, }}$ is more effective than random collaborator selection method. Moreover, we found that the differences between $\mathrm{NiCS}_{\text {andom }}$ and collaborator selection method based on similarity of shared input variables are statistically indistinguishable. 
Table 5.7: WBAN settings for testing Multilevel FLCs designed regarding target $\mathrm{T}_{2}$.

\begin{tabular}{|c|l|l|}
\hline Scenario & No of sensors & WBAN setting \\
\hline 1 & One sensor & Temperature \\
\hline 2 & Two sensors & Temperature and ECG \\
\hline 3 & Three sensors & Temperature, ECG and respiratory rate \\
\hline 4 & Four sensors & Temperature, ECG, respiratory rate and HR \\
\hline 5 & Five sensors & Temperature, ECG, respiratory rate, HR and EEG \\
\hline 6 & Six sensors & $\begin{array}{l}\text { Temperature, ECG, EEG, respiratory rate, HR, } \\
\text { and BP }\end{array}$ \\
\hline 7 & Seven sensors & $\begin{array}{l}\text { Temperature, ECG, EEG, respiratory rate, HR, BP } \\
\text { and Motion }\end{array}$ \\
\hline 8 & Eight sensors & $\begin{array}{l}\text { Temperature, ECG, EEG, respiratory rate, HR, BP, } \\
\text { Motion and Endoscope Imaging }\end{array}$ \\
\hline 9 & Nine sensors & $\begin{array}{l}\text { Temperature, ECG, EEG, respiratory rate, HR, BP, } \\
\text { Motion, Endoscope Imaging and ECG }\end{array}$ \\
\hline 10 & Nine sensors & $\begin{array}{l}\text { Temperature, ECG, EEG, respiratory rate, HR, BP, } \\
\text { Motion, Endoscope Imaging, ECG and ECG }\end{array}$ \\
\hline
\end{tabular}

\section{Comparison between CPSO-based Multilevel FLC Design (Target $\mathrm{T}_{2}$ ) and Competing Algorithms}

After comparing CoCLF-MAC $T_{2}$ with competing algorithms including CoCLF-MAC $_{T_{1}}$ on the testing results (see Tables 5.9, 5.10, 5.11 and 5.12), we found that CoCLF-MAC $T_{T_{2}}$ can generally outperform IEEE 802.15.4 and other competing algorithms in both PDR and packet delay. In other words, CoCLF-MAC $T_{2}$ is the only algorithm which can provide higher PDR and throughput than others without incurring longer delay. Moreover, it produces a lower collision rate than others.

Table 5.9 shows that PDR is almost $100 \%$ when the network consists of only two nodes including the coordinator. When the traffic load increases by adding more sensor nodes, PDR starts to decrease. Table 5.9 shows that CoCLF-MAC $T_{T_{2}}$ can manage to achieve higher PDR than IEEE 802.15.4, AGA+IEEE 802.15.4, AGA+CLFB, FCMA and CoCLF-MAC $T_{T_{1}}$, except in 
Table 5.8: Training results obtained by CPSO in terms of PDR and packet delay in seconds $(\lambda=2.5)$

\begin{tabular}{|c|c|c|c|c|c|c|}
\hline $\begin{array}{l}\text { Training } \\
\text { Scenario }\end{array}$ & PM & gBest & Random & $\mathrm{NiCS}_{g B e s t}$ & $\mathrm{NiCS_{ \text {random } }}$ & Similarity Factor \\
\hline \multirow{4}{*}{1} & \multirow{2}{*}{ PDR } & 0.985 & 0.984 & 0.987 & 0.988 & 0.978 \\
\hline & & \pm 0.001 & \pm 0.002 & \pm 0.002 & \pm 0.001 & \pm 0.003 \\
\hline & \multirow{2}{*}{ Delay } & 0.111 & 0.111 & 0.111 & 0.111 & 0.112 \\
\hline & & \pm 0.001 & \pm 0.001 & \pm 0.001 & \pm 0.001 & \pm 0.001 \\
\hline \multirow{4}{*}{2} & \multirow{2}{*}{ PDR } & 0.978 & 0.979 & 0.982 & 0.982 & 0.978 \\
\hline & & \pm 0.001 & \pm 0.004 & \pm 0.001 & \pm 0.003 & \pm 0.004 \\
\hline & \multirow{2}{*}{ Delay } & 0.112 & 0.112 & 0.111 & 0.111 & 0.112 \\
\hline & & \pm 0.001 & \pm 0.002 & \pm 0.001 & \pm 0.001 & \pm 0.001 \\
\hline \multirow{4}{*}{3} & \multirow{2}{*}{ PDR } & 0.589 & 0.562 & 0.648 & 0.566 & 0.575 \\
\hline & & \pm 0.002 & \pm 0.001 & \pm 0.001 & \pm 0.002 & \pm 0.003 \\
\hline & \multirow{2}{*}{ Delay } & 3.042 & 3.127 & 2.530 & 3.056 & 3.134 \\
\hline & & \pm 0.002 & \pm 0.001 & \pm 0.002 & \pm 0.004 & \pm 0.004 \\
\hline \multirow{4}{*}{4} & \multirow{2}{*}{ PDR } & 0.454 & 0.449 & 0.535 & 0.454 & 0.454 \\
\hline & & \pm 0.002 & \pm 0.001 & \pm 0.001 & \pm 0.006 & \pm 0.004 \\
\hline & \multirow{2}{*}{ Delay } & 4.167 & 4.134 & 3.170 & 4.039 & 4.019 \\
\hline & & \pm 0.001 & \pm 0.003 & \pm 0.001 & \pm 0.004 & \pm 0.003 \\
\hline \multirow{4}{*}{5} & \multirow{2}{*}{ PDR } & 0.384 & 0.367 & 0.407 & 0.376 & 0.383 \\
\hline & & \pm 0.002 & \pm 0.002 & \pm 0.001 & \pm 0.002 & \pm 0.004 \\
\hline & \multirow{2}{*}{ Delay } & 4.546 & 4.736 & 4.158 & 4.641 & 4.598 \\
\hline & & \pm 0.002 & \pm 0.005 & \pm 0.002 & \pm 0.003 & \pm 0.004 \\
\hline
\end{tabular}


scenarios 3 and 5. In these two scenarios, the competing algorithms could manage to achieve higher PDR by imposing significantly longer backoffs. In other words, the results clearly show CoCLF-MAC $T_{T_{2}}$ is more reliable than IEEE 802.15.4 without imposing longer backoffs on sensor nodes.

Generally, by increasing the number of nodes, PDR will decrease in all algorithms. This is because of the increasing number of collisions on the network, as shown in Table 5.10. CoCLF-MAC $T_{2}$ can achieve a lower collision rate than AGA+IEEE 802.15.4, AGA+CLFB, FCMA and CoCLF$\mathrm{MAC}_{T_{1}}$, except in scenarios 3 and 5. However, in scenarios 3 and 5, long backoff delays are applied to avoid collisions. On the other hand, CoCLF$\mathrm{MAC}_{T_{2}}$ can perform much better than IEEE 802.15.4 in term of collision rate without sacrificing communication delay.

As mentioned before, MAC throughput of the network presented in Table 5.11 is indirectly improved by increasing PDR in WBANs. Consequently, CoCLF-MAC $_{T_{1}}, \mathrm{AGA}+\mathrm{CLFB}$ and FCMA can outperform CoCLF-MAC $T_{T_{2}}$ in terms of throughput in some scenarios (e.g. scenarios 3 and 5). Meanwhile, our statistical analysis shows that CoCLF-MAC $T_{2}$ statistically outperforms IEEE 802.15.4 when there are more than two sensor nodes in the network.

Increasing the number of nodes lead to an increase in the network congestion. Accordingly, IEEE 802.15.4 increases the backoff delays to reduce the collision rate. Inducing longer backoff delays result in longer packet delays in the network as shown in Table 5.12. Although the results show CoCLF-MAC $T_{1}, \mathrm{AGA}+\mathrm{CLFB}$ and FCMA can occasionally achieve higher PDR and throughput than CoCLF-MAC $T_{T_{2}}$, it is important to note that they sacrifice the packet delay hugely, which means they are undesirable for delay sensitive WBAN applications. For example, in scenario 5, CoCLF-MAC $T_{1}$ and FCMA introduce significantly longer delay than CoCLF-MAC $_{T_{2}}$, as shown in Table 5.12. In other words, CoCLF-MAC $T_{T_{2}}$ can outperform IEEE 802.15.4, AGA+IEEE 802.15.4, AGA+CLFB, FCMA and CoCLF-MAC $T_{1}$ in term of the packet delay. Particularly, ANOVA and Tukey's post-hoc analysis indicate that the delay achieved by CoCLF- 
$\mathrm{MAC}_{T_{2}}$ is statistically significantly less than IEEE 802.15.4, AGA+IEEE 802.15.4, AGA+CLFB, FCMA and CoCLF-MAC $T_{T_{1}}$, when there are more than two sensor nodes in the network.

\subsection{Chapter Summary}

In this chapter, we proposed an EC-based approach to automatically design Fuzzy Logic Controllers (FLCs) for Cooperative Cross Layer Fuzzy MAC (CoCLF$\mathrm{MAC}$ ) in WBANs. Different from many works in the literature that utilised FLCs only at the sensor level, CoCLF-MAC explored and successfully demonstrated the importance of utilising FLCs both at the sensor level and the coordinator level. We also utilised two separate design targets to achieve high network reliability and performance in WBANs. The first target is designing effective multilevel FLCs (i.e. CoCLF-MAC $T_{1}$ ) to provide reliability and performance for a specific WBAN setting. On the other hand, the second target aims to design desirable multilevel FLCs (i.e. CoCLF$\mathrm{MAC}_{T_{2}}$ ) for more general WBAN configurations.

Meanwhile, we examined the usefulness of the well-know Cooperative PSO (CPSO) with five different collaborator selection methods. We showed that CPSO can successfully design both the sensor and coordinator control levels jointly to improve reliability and performance of WBANs. We also confirmed that our proposed collaborator selection methods is more effective than conventional collaborator selection methods in design multilevel FLCs in WBANs.

Moreover, we have confirmed that CoCLF-MAC $T_{T_{1}}$ can achieve significantly better reliability and performance than cutting-edge algorithms such as IEEE 802.15.4, AGA+ IEEE 802.15.4, AGA+CLFB, FCMA, CLFB and $\mathrm{D}^{2} \mathrm{MAC}$. We have also experimentally shown that only CoCLF-MAC $T_{T_{2}}$ can outperform IEEE 802.15.4 in terms of both PDR and packet delay for a wider range of communication scenarios.

It is worthwhile mentioning that, during the whole design process, we 


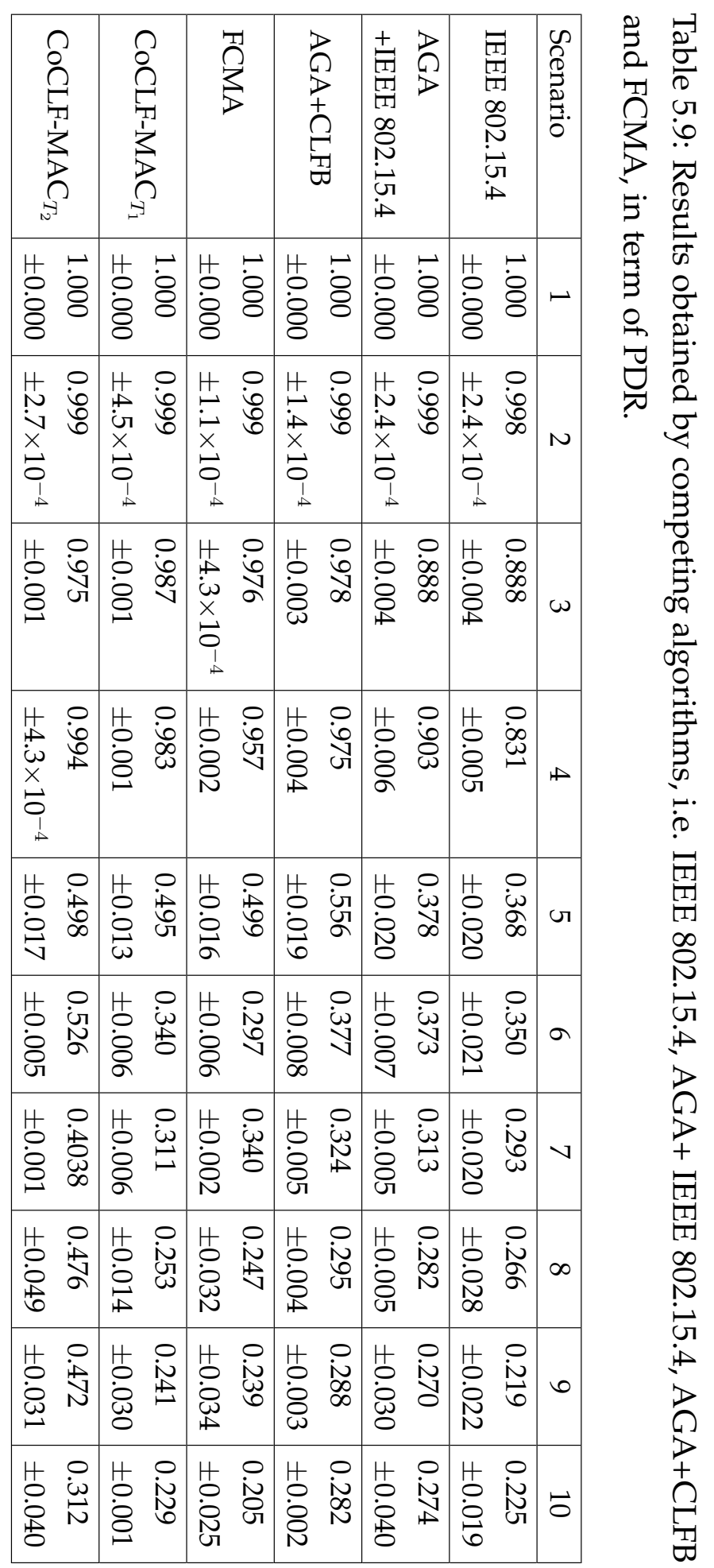




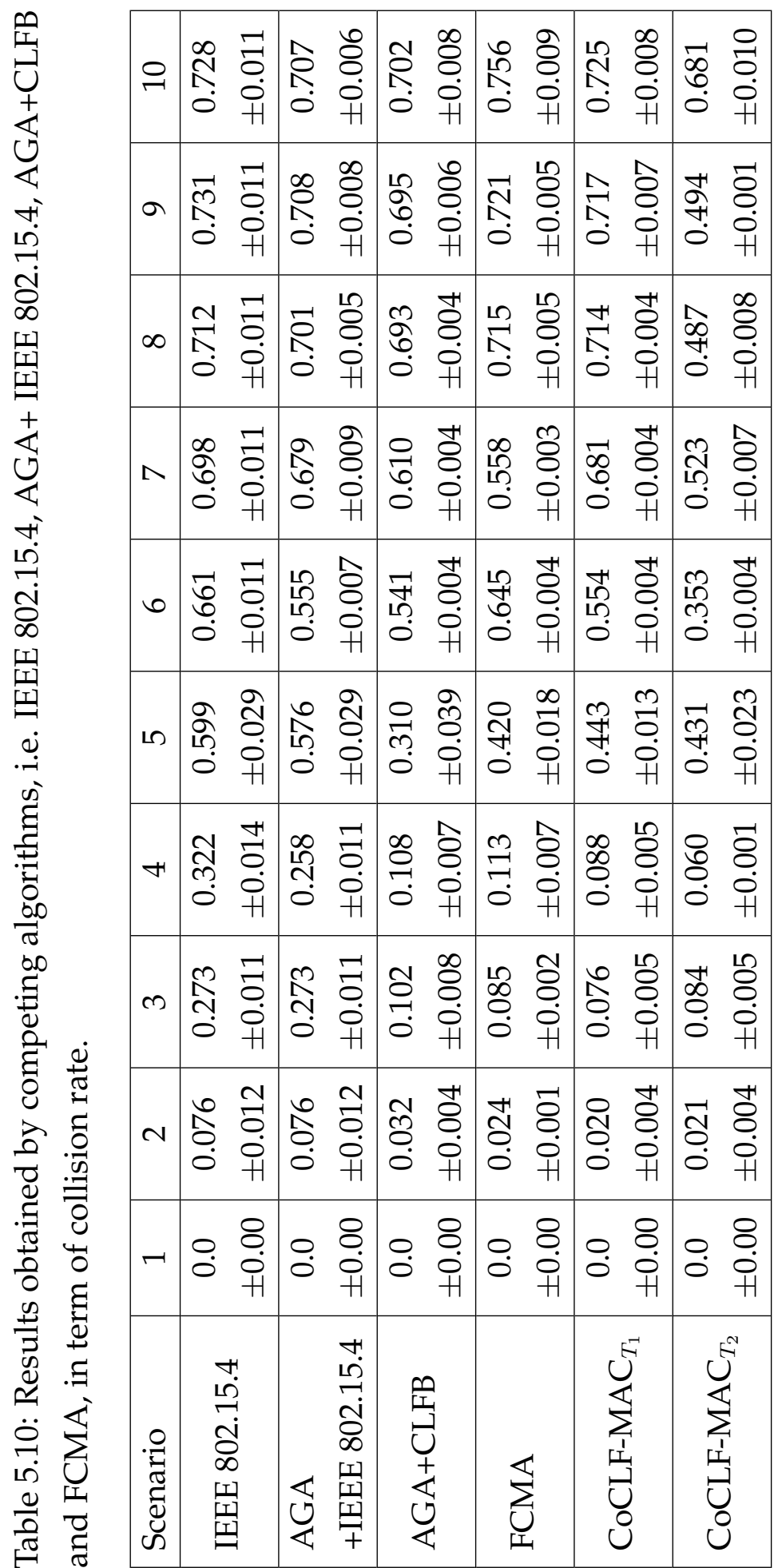




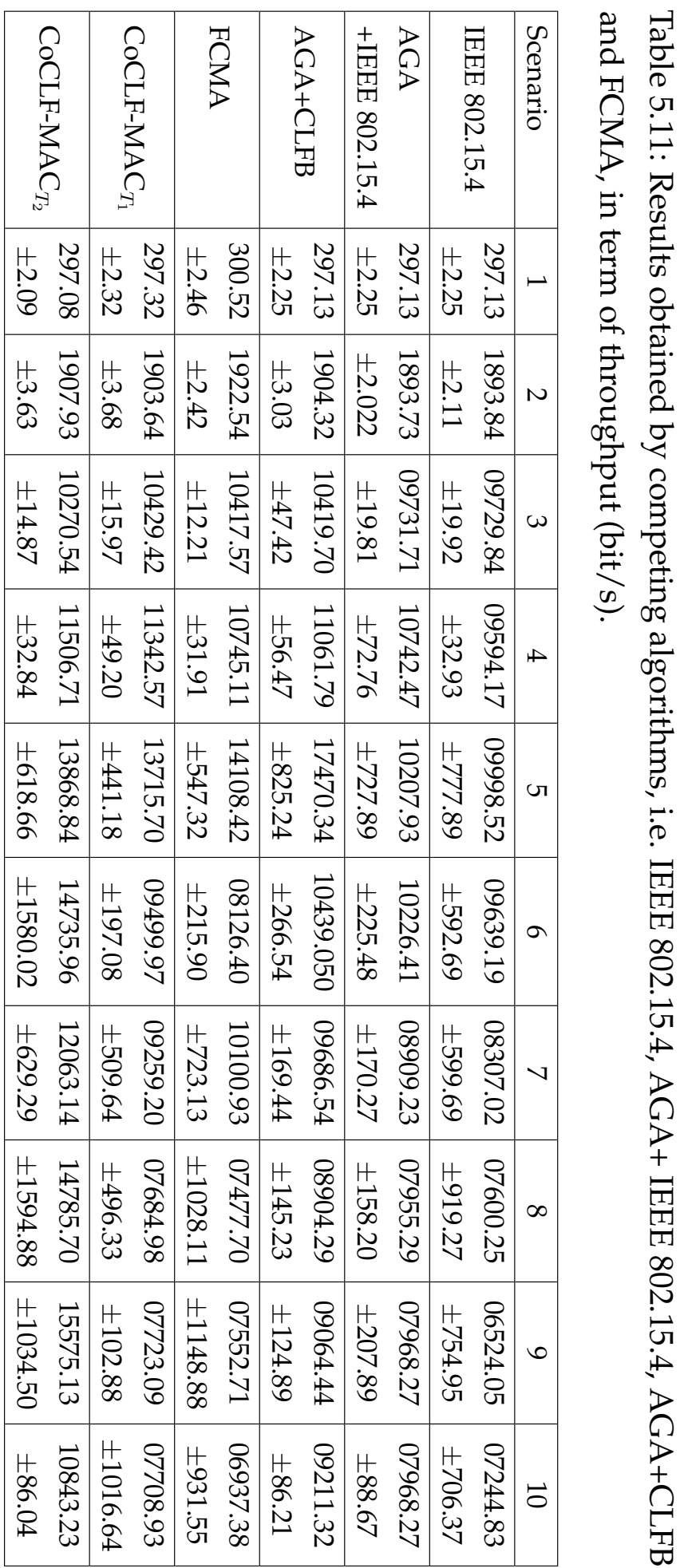




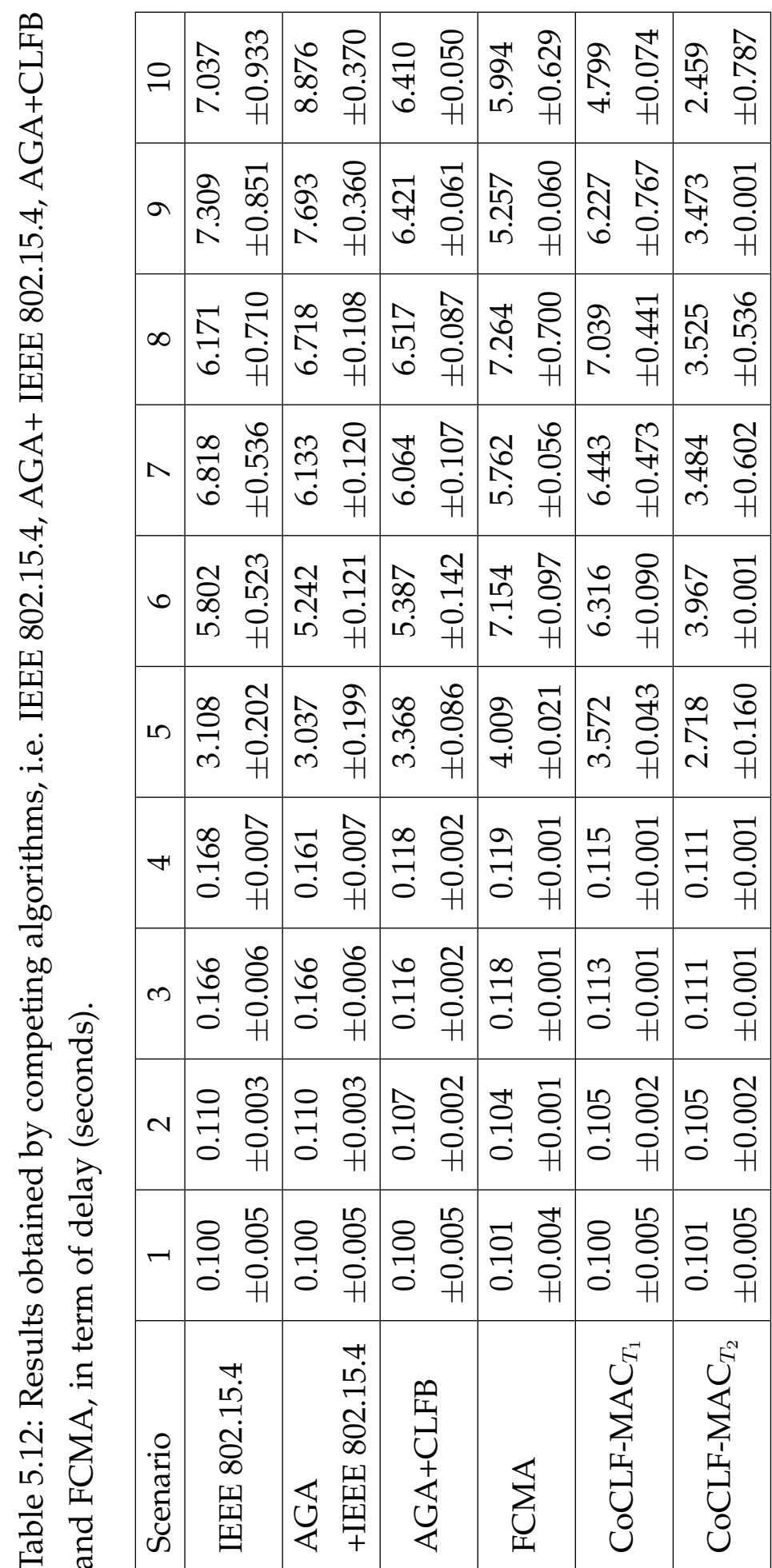


have introduced neither specific mechanism nor significant changes to IEEE 802.15.4. FLCs are fuzzy rules that are simple and easy to process. Once FLCs for CoCLF-MAC have been designed through our cooperative automatic approach, they can be easily integrated into sensor nodes and the coordinator as long as they are compatible with IEEE 802.15.4 without any major changes. 


\section{Chapter 6}

\section{Conclusions}

This chapter highlights the achieved objectives, main conclusions, and future research directions. The overall goal of this thesis is to investigate cross-layer fuzzy logic controllers to improve reliability and performance of MAC protocols in WBANs. This goal was successfully achieved by (1) effectively using cross-layer based FLCs to improve reliability and performance of IEEE 802.15.4 based MAC protocols, (2) developing evolutionary approaches for automatic design of FLCs for cross-layer MAC in WBANs in order to assure a reasonable balance between reliability and performance, and (3) developing a two-level cross-layer FLC scheme for IEEE 802.15.4 based MAC protocols to take advantages of both distributed and centralised mechanisms, i.e at both sensor level and coordinator level, to cooperatively enhance channel quality.

The proposed design approaches were evaluated and compared with related state-of-the-art algorithms. The results showed that our approaches in this thesis are highly competitive and significantly outperformed the related state-of-the-art algorithms.

The remainder of this chapter is organised as follows: Section 6.1 provides achieved objectives of each chapter. Section 6.2 presents conclusions and discussion of each chapter. Section 6.3 highlights future research directions. 


\subsection{Achieved Research Objectives}

This thesis has successfully achieved its three main objectives as follows:

1. This thesis developed a Cross-Layer Fuzzy logic based Backoff (CLFB) scheme to control contention based channel access. This new scheme provides a desirable balance between reliability and performance by introducing a distributed cross-layer FLC architecture to improve exponential backoff algorithm based on channel condition and application requirements. Particularly, CLFB uses the recent channel condition to predict desirable backoff exponent in the CSMA/CA algorithm. It also employs application data rates to balance between the waiting time and the channel condition. Furthermore, a manual approach has been adopted to design the FLC in our CLFB. The simulation results clearly showed that CLFB significantly outperformed the competing algorithms, including IEEE 802.15.4, Additional Carrier Sensing (ACS) [163] and Dynamic delayed Medium Access Control ( $\mathrm{D}^{2} \mathrm{MAC}$ ) [107] as well as a recently proposed algorithm [31] termed "NB-Step", in terms of reliability and performance without having negative impact on packet delays. A part of this objective has been published in [113].

2. This thesis developed an automatic EC-based approach for effective and efficient design of FLCs for MAC in WBANs. With the goal of improving network reliability while keeping the communication delay at a low level, we have defined three coding schemes and evaluated their usefulness during the proposed evolutionary design process. We have also examined the influence of fitness functions that measure the effectiveness of each possible FLC design in order to achieve a good balance between network reliability and performance. Moreover, we have utilized surrogate models to improve the efficiency of the design process. In consideration of practical usefulness, we have 
further identified two main design targets. The first target is to design effective FLCs for a specific network configuration. The second target focuses on designing FLCs to function effectively across a wide range of network settings. Through a comprehensive set of experiments, we have finally shown that our proposed approach for designing FLCs clearly outperformed several recently proposed algorithms for media access control in WBANs, including IEEE 802.15.4, ACS [163], $\mathrm{D}^{2} \mathrm{MAC}$ [107] and NB-Step [31]. A part of this objective has been published in $[115,114]$.

3. This thesis developed a Cooperative Cross Layer Fuzzy MAC controller (CoCLF-MAC) which employs a two-level cross-layer FLC scheme for IEEE 802.15.4 based MAC protocols. The new scheme takes the advantage of both distributed and centralised mechanisms, at both the sensor level and coordinator level to effectively enhance the reliability and performance of WBANs. Particularly, the CLFB at the sensor level controls the contention based channel access, and Cross-Layer Fuzzy logic based GTS Allocation controller (CLFGA) at the coordinator level controls contention free channel access.

This thesis also developed an automatic approach for cooperative and effective design of our two-level FLC by using Cooperative CoEvolutionary Algorithms (CCEAs) in particular Cooperative PSO (CPSO). For this purpose, we proposed a new collaborator selection scheme based on our insight into efficient communication in WBANs and employed the network knowledge to select suitable collaborators in CPSO. We found that our new collaborator selection method can clearly improve the effectiveness of the design process. We finally experimentally showed that our proposed approach for designing two-level FLCs can clearly outperform several recently proposed algorithms for media access control in WBANs, including IEEE 802.15.4 [3], CLFB, D²MAC [107], Adaptive GTS Allocation (AGA)+ 
IEEE 802.15.4 [64], AGA+CLFB [64, 115] and Fuzzy Control Medium Access (FCMA) [177]. A part of this achieved has been published in [116].

\subsection{Main Conclusions}

Overall, this thesis finds that cross-layer FLCs can effectively improve the communication quality in IEEE 802.15.4 based WBANs. This thesis also introduces evolutionary approaches to automatically and effectively design our proposed FLCs in order to assure a reasonable balance between reliability and performance. These new approaches are experimentally evaluated and shown to outperform several state-of-the-art algorithms.

\subsubsection{Cross-Layer Fuzzy Logic based Backoff}

Chapter 3 introduced a cross-layer FLC for IEEE 802.14.4 based MACs, called Cross-Layer Fuzzy logic based Backoff system (CLFB), to improve both network reliability and performance. Contrary to existing algorithms, which increase either the WBAN reliability or performance, the new approach explicitly considers both of the two essential requirements. Particularly, in consideration of channel condition and application requirements, CLFB can improve reliability and performance without increasing packet delay. By integrating our CLFB into the IEEE 802.15.4 MAC sub-layer, we successfully enhanced the competence of this IEEE standard for various WBAN-based applications. Moreover, FLCs are some simple and easy to process fuzzy rules. Therefore, once FLCs are designed, they can be easily integrated into sensor nodes without increasing the communication complexity. Since CLFB focuses on tuning the parameter of the exponential backoff algorithm in IEEE 802.15.4, it minimises the modifications to the standard and does not introduce new communication overheads. This chapter also experimentally demonstrated the effectiveness of CLFB over 
several cutting-edge algorithms in terms of both network reliability and performance.

\subsubsection{Automatic Design of Fuzzy Logic Controllers}

Chapter 4 introduced a new EA-based approach for fully automated design of FLCs for CLFB in WBANs. This chapter introduced our approach through five steps: 1) encoding the candidate solutions, 2) defining the fitness function, 3) defining the fitness evaluation method, 4) applying EAs to find suitable candidate solution that maximises the fitness function, and finally 5) utilizing surrogate models to improve the efficiency of the evolutionary design process.

1. Encoding the candidate solutions: This step of the design process focuses on the coding scheme. The coding scheme turns an arbitrary design of FLC into a candidate solution that can be evolved by EAs. In order to produce an effective design of FLCs, we jointly investigate the efficacy of three different coding schemes, namely UnRestricted Coding Scheme (URCS), Strictly Restricted Coding Scheme (SRCS) and the Moderately Restricted Coding Scheme (MRCS), with varied levels of restrictions. Despite interpretability issues, we showed that the performance of URCS is better than SRCS and MRCS. Although historically more priority has been given to the performance, the importance of interpretability is also well recognised. We demonstrated that our coding schemes (i.e. SRCS and MRCS) can provide a desirable trade-off between interpretability and performance in practice.

2. Fitness function: In this step of the design process, we have designed a meaningful fitness function to guide the evolutionary design process. Our main design goal is to optimise the reliability of WBANs (measured by PDR). On the other hand, the performance of the network also has to be competitive to IEEE 802.15.4. In this research, 
throughput is considered indirectly through enhancing PDR. However, increasing PDR may lead to an increase in packet delay. Consequently, PDR is constrained to packet delay. We, therefore, have to pursue PDR as the main objective and packet delay as a constrained the violation of which will be penalised in the fitness function.

3. Fitness evaluation method: In order to improve the practicality of our approach, we have defined two evaluation methods in regard to two different design targets. The first design target is to design FLCs that function effectively on a specific network setting. The second target is to design FLCs that are able to work consistently well across a wide range of network settings.

4. Applying ECs: In the literature, it has been frequently shown that EC techniques are very effective at handling noisy and multi-modal problems. Due to this reason, EC-based automatic design of FLCs is evaluated comprehensively in this research. It is worth noticing that our approach is flexible to work with many EC techniques. Therefore, in order to demonstrate that our approach can effectively work with different EC techniques, we have successfully investigated the overall usefulness of the approach through two well-known EC techniques, i.e. PSO and DE.

5. Surrogate-assisted ECs: Since evaluating the fitness of any candidate FLC design is time-consuming, this thesis also improved the efficiency of the design process through surrogate models. It is worthwhile to mention that, although the computation time still seems high after using the surrogate model, the designed FLCs will actually be used repeatedly in many WBAN applications. In other words, we need to design FLCs only once and reuse them for many applications.

The practicality of the approach for designing FLCs demands for three different requirements: effectiveness, efficiency and general applicability. 
Based on the reasons given below we believe that our approach is highly practical.

1. Effectiveness: We have successfully demonstrated, through experimental results, that our approach can design FLCs effectively to provide both reliability and performance on WBANs. Particularly, we have compared CLFB using our automated designed FLCs to other state-of-art algorithms. The comparison shows that FLCs design through our approach can outperform other competing algorithms in terms of both reliability and performance.

2. Efficiency: The computation time of designing FLCs is considered as the efficiency of the approach. We have enhanced the efficiency of the approach through surrogate models. In this way, we have experimentally shown that our automatic design approach significantly improves the efficiency of the design process.

3. General applicability: We introduced two design targets. The first target is for patients who have a specific WBAN configuration. The second target focuses on multiple WBAN configurations. Although the designed rules based on the second target in a specific WBAN setting might not as effective as the designed rules based on the first target, the second target can provide a reasonably good balance between PDR and delay for a wide range of WBAN settings. That means this is not necessary to re-design rules from scratch for every different network setting. In this way, a specific sensor node can be added or removed without significantly degrading reliability and performance of the WBAN. 


\subsubsection{Cooperative Design of Two Level Fuzzy Logic Con- trollers}

Chapter 5 introduced a two-level cross-layer FLC, which is called Cooperative Cross Layer Fuzzy MAC controller (CoCLF-MAC), for high quality communication in IEEE 802.15.4-based WBANs. Specifically, CLFB at the sensor level aims at optimising the performance of exponential backoff algorithm. Meanwhile, our Cross-Layer Fuzzy logic based GTS Allocation controller (CLFGA) at the coordinator level prioritises GTS allocation based on channel condition and application data rate. The chapter finds that the proposed CoCLF-MAC has potential to significantly improve reliability and performance of the IEEE 802.15.4-based MAC in the context of WBANs.

To cooperatively and automatically design FLCs at both the sensor level and coordinator level, a CCEA-approach has been introduced in this chapter. This chapter finds that collaborator selection can significantly affect the effectiveness of CCEAs in the design process. Therefore, we introduce two collaborator selection methods based on our knowledge for effective communication in WBANs. It has been shown that the proposed collaborator selection methods can clearly improve the effectiveness of the design process.

This chapter also has studied the practicality of the design approach as follows:

1. Effectiveness: The experimental results showed that our automatic approach to design FLCs in CoCLF-MAC can significantly outperform other cutting-edge algorithms in terms of both network reliability and performance.

2. General applicability: CoCLF-MAC has been designed based on two design targets: (1) a specific WBAN configuration (2) over multiple WBAN configurations. Both targets have been achieved successfully, confirming the practical usefulness of the proposed approach. 


\subsection{Future Works}

This section suggests a direction of future research works.

\subsubsection{IEEE 802.15.6}

Since most WBANs are often supported by IEEE 802.15.4 compliant chip set $[91,83]$, this thesis has focused on improving IEEE 802.15.4. Throughout, we have shown that cross-layer FLCs can effectively improve the reliability and performance of IEEE 802.15.4 based WBANs. However, the IEEE 802.15 task group 6 [4] recently introduced IEEE 802.15.6 to reduce low energy consumption and improve reliable communication in, on, or around human body. Although IEEE 802.15.6 outlines the basic elements needed to provide interoperability among different devices [140], it does not consider a complete MAC protocol that is suitable for WBANs. For instance, it provides packet format and message exchange protocols. However, this standard does not consider perceived policies regarding the channel condition history and application requirements, resulting in an incomplete MAC protocol. In order to consider the channel condition history and application requirements, it is important to also investigate using cross-layer FLCs in IEEE 802.15.6 to improve reliability and performance of the network.

\subsubsection{Multi-Objective EC Techniques}

In this thesis, we focused on improving network reliability and performance in WBANs through a single optimisation objective. The objective focuses on optimising the reliability of WBANs (measured by PDR). As a result, throughput can be increased directly through increasing PDR. On the other hand, the performance of the network has to remain competitive with respect to IEEE 802.15.4. However, increasing PDR may lead to an increase in packet delay. We therefore decided to pursue PDR as the main objective and packet delay as a soft constraint, the violation of which would be 
penalised through the fitness function.

Rather than optimising one objective, in the future, we can consider the use of Evolutionary Multi-Objective (EMO) algorithms such as, Nondominated Sorting Genetic Algorithm II (NSGA-II) [37] and Strength Pareto Evolutionary Algorithm 2 (SPEA-2) [180]. Through EMO algorithms, we can pursue simultaneously many different compromises over two conflicting objectives, i.e. PDR and packet delays. Selecting the most suitable FLC design over a group of Pareto-optimal designs suggests another interesting research question to be investigated.

\subsubsection{Surrogate-assisted Cooperative Coevolution}

Since fitness evaluations may often become costly and EC techniques usually require a large number of fitness function evaluations before converging to a good FLC design [74], it is necessary to utilise a surrogate model for approximating the fitness of candidate to increase the practicality of our design approach. Therefore, in this thesis, we have investigated the usefulness of surrogate models to improve the efficiency of conventional EC-based design of FLCs for CLFB in WBANs. We experimentally showed that, there is an almost $70 \%$ saving of computation time upon using surrogate models and they are as effective as design approach without surrogate models. Nevertheless, the computation time for our cooperative coevolutionary design of multilevel FLCs for CoCLF-MAC in WBANs is $489.96 \pm 6.42$ hours. In order to improve the efficiency and practicality of our cooperative coevolutionary design process, it is important to use surrogate models in future approach.

Furthermore, in this thesis, we use have used Multi-Layer Perceptrons (MLPs) to build our surrogate model. Nevertheless, other models approximation models can also be utilised in our design problem. For example, statistical learning methods such as Gaussian processes can provide accuracy information such as confidence intervals for the estimated values. On the other hand, the computation cost of Radial Basis Function (RBF) models 
are less expensive than Gaussian processes. To build an effective surrogate model to assist EC-based design of FLCs, it is important to study different approximation models in our design problem in the future.

\subsubsection{Real Life Experiments}

In this thesis, we confirmed the effectiveness of our schemes through network simulations based on $\mathrm{OMNeT}++$. Nevertheless, our simulations do not always reflect real-world scenarios precisely. In order to evaluate our proposed schemes such as CLFB and CoCLF-MAC, in the future, we are interested in examining them in real-world testbed. Real-world evaluation is challenging due to three requirements: (1) Medical and Human Ethics Committee (HEC) approvals, which are needed to conduct tests on patients. (2) Selection of representative patients and test scenarios. (3) Finally, equipment preparation and implementation require funding. Due to these reasons, we could not manage to perform real-life tests in this thesis. However, we ae planning to seek funding to evaluate our proposed schemes through real-life tests, in the future.

\subsection{Final Remarks}

The Human population took thousands of generations to reach 2.3 billion in 1945. However, in one human lifetime, it went to 6.4 billion in 2005 and the demographic expectation is over 9 billion in 2050 [153]. This puts pressure on food, water and vulnerable natural resources, health care demand and medical treatment. Hence there is a growing need for more scalable and more inexpensive healthcare solutions [106]. Advances in sensor networking have opened up new opportunities in healthcare systems by introducing Wireless Body Area Networks (WBANs). This thesis has improved quality of communication in WBANs based on the current standard (i.e. IEEE 802.15.4). We hope this research could result in long-term benefits of using WBANs including earlier crisis detection and 
help to improve quality of life for people. 


\section{Bibliography}

[1] Fuzzy Logic in Process Control. Springer Berlin Heidelberg, Berlin, Heidelberg, 2005, pp. 97-123.

[2] Health informatics - PoC medical device communication - part 00101: Guide-guidelines for the use of RF wireless technology. IEEE Std 11073-00101-2008 (2008), 1-99.

[3] IEEE Standard for local and metropolitan area networks-part 15.4: Low-Rate Wireless Personal Area Networks (LR-WPANs). IEEE Std 802.15.4-2011 (Revision of IEEE Std 802.15.4-2006) (Sept 2011), 1-314.

[4] IEEE standard for local and metropolitan area networks - part 15.6: Wireless body area networks. IEEE Std 802.15.6-2012 (2012), 1-271.

[5] OMNeT++ network simulation framework, December 2014.

[6] Ai, T. J., And Kachitvichyanukul, V. Particle swarm optimization and two solution representations for solving the capacitated vehicle routing problem. Comput. Ind. Eng. 56, 1 (Feb. 2009), 380-387.

[7] Alba, E., Garcia-Nieto, J., Jourdan, L., And Talbi, E. G. Gene selection in cancer classification using $\mathrm{pso} / \mathrm{svm}$ and ga/svm hybrid algorithms. In 2007 IEEE Congress on Evolutionary Computation (Sept 2007), pp. 284-290. 
[8] ALBALT, M., AND NASIR, Q. Adaptive backoff algorithm for ieee 802.11 mac protoco. International Journal of Communications, Network and System Sciences 2, 4 (2009), 300-317.

[9] Alonso, J. M., Castiello, C., And Mencar, C. Interpretability of Fuzzy Systems: Current Research Trends and Prospects. Springer Berlin Heidelberg, 2015, pp. 219-237.

[10] AlRashidi, M. R., AND El-Hawary, M. E. A survey of particle swarm optimization applications in electric power systems. IEEE Transactions on Evolutionary Computation 13, 4 (Aug 2009), 913-918.

[11] Bachache, N., AND Wen, J. Design fuzzy logic controller by particle swarm optimization for wind turbine. In Advances in Swarm Intelligence, Y. Tan, Y. Shi, and H. Mo, Eds., vol. 7928 of Lecture Notes in Computer Science. Springer Berlin Heidelberg, 2013, pp. 152-159.

[12] BACK, T., HAMmel, U., AND SChWefel, H. P. Evolutionary computation: comments on the history and current state. IEEE Transactions on Evolutionary Computation 1, 1 (Apr 1997), 3-17.

[13] BALDO, N., AND ZORZI, M. Fuzzy logic for cross-layer optimization in cognitive radio networks. IEEE Communications Magazine 46, 4 (April 2008), 64-71.

[14] Baturone, I., Moreno-Velo, F. J., Sanchez-Solano, S., AND Ollero, A. Automatic design of fuzzy controllers for car-like autonomous robots. IEEE Transactions on Fuzzy Systems 12, 4 (Aug 2004), $447-465$.

[15] Berthold, F., Ravey, A., Blunier, B., Bouquain, D., Williamson, S., AND Miraoui, A. Design and development of a smart control strategy for plug-in hybrid vehicles including vehicle-to-home functionality. IEEE Transactions on Transportation Electrification 1, 2 (Aug 2015), 168-177. 
[16] BeYer, H.-G., AND SCHWefel, H.-P. Evolution strategies \&ndash;a comprehensive introduction. 3-52.

[17] BiNGÜL, Z., AND KARAHAN, O. A fuzzy logic controller tuned with PSO for 2 DOF robot trajectory control. Expert Systems with Applications 38, 1 (2011), 1017 - 1031.

[18] Bouallégue, S., Haggge, J., Ayadi, M., and Benrejeb, M. Pidtype fuzzy logic controller tuning based on particle swarm optimization. Engineering Applications of Artificial Intelligence 25, 3 (2012), 484 493.

[19] Bouarroudj, N., Boukhetala, D., DJARI, A., Rais, Y., AND BENLAHBIB, B. Flc based gaussian membership functions tuned by pso and ga for mppt of photovoltaic system: A comparative study. In 2017 6th International Conference on Systems and Control (ICSC) (May 2017), pp. 317-322.

[20] Bradai, N., Fourati, L. C., AND KAMOUN, L. Investigation and performance analysis of $\{\mathrm{MAC}\}$ protocols for $\{\mathrm{WBAN}\}$ networks. Journal of Network and Computer Applications 46 (2014), 362 - 373.

[21] BuLL, L. On model-based evolutionary computation. Soft Computing 3, 2 (Sep 1999), 76-82.

[22] Carpenter, W. C., And Barthelemy, J. F. M. A comparison of polynomial approximations and artificial neural nets as response surfaces. Structural optimization 5, 3 (1993), 166-174.

[23] Casillas, J., Cordon, O., Del Jesus, M., and Herrera, F. Genetic tuning of fuzzy rule deep structures preserving interpretability and its interaction with fuzzy rule set reduction. IEEE Transactions on Fuzzy Systems 13, 1 (Feb 2005), 13-29. 
[24] Cavalcanti, D., Schmitt, R., And Soomro, A. Performance analysis of 802.15.4 and 802.11e for body sensor network applications. In 4th International Workshop on Wearable and Implantable Body Sensor Networks (BSN 2007), S. Leonhardt, T. Falck, and P. Mähönen, Eds., vol. 13 of IFMBE Proceedings. Springer Berlin Heidelberg, 2007, pp. 914.

[25] Cavalcanti, D., Schmitt, R., And Soomro, A. Performance Analysis of 802.15.4 and 802.11e for Body Sensor Network Applications. Springer Berlin Heidelberg, Berlin, Heidelberg, 2007, pp. 9-14.

[26] Cavallari, R., Martelli, F., Rosini, R., Buratti, C., And VerDONE, R. A survey on wireless body area networks: Technologies and design challenges. IEEE Communications Surveys Tutorials 16, 3 (Third 2014), 1635-1657.

[27] CHANG, C., AND XU, D. Differential evolution based tuning of fuzzy automatic train operation for mass rapid transit system. IEE Proceedings Electric Power Applications 147, 3 (May 2000), 206-212.

[28] CHEN, C.-L., AND CHANG, M.-H. Optimal design of fuzzy slidingmode control: A comparative study. Fuzzy Sets and Systems 93, 1 (1998), $37-48$.

[29] Chen, G., Low, C. P., AND YAnG, Z. Preserving and exploiting genetic diversity in evolutionary programming algorithms. IEEE Transactions on Evolutionary Computation 13, 3 (June 2009).

[30] Chen, M., Gonzalez, S., Vasilakos, A., CaO, H., And LeunG, V. C. Body area networks: A survey. Mobile Networks and Applications 16, 2 (2011), 171-193.

[31] Chen, M., ZHU, X., AND ZHU, H. Service adaptively medium access control algorithm based on fuzzy logical for energy harvesting wireless sensor networks. Journal of Networks 9, 9 (2014). 
[32] Ching-Chang, W., Hou-Yi, W., And Shih-An, L. PSO-based motion fuzzy controller design for mobile robots. International Journal of Fuzzy Systems 10, 1 (2008), $284-292$.

[33] Chu, H.-T., HuAnG, C.-C., LiAn, Z.-H., AND Tsai, J. J. P. A ubiquitous warning system for asthma-inducement. In IEEE International Conference on Sensor Networks, Ubiquitous, and Trustworthy Computing (SUTC'06) (June 2006), vol. 2.

[34] Cordón, O., Herrera, F., Hoffmann, F., And Magdalena, L. Fuzzy Rule-Based Systems. WORLD SCIENTIFIC, 2001, pp. 1-46.

[35] DABHI, V. K., AND ChaUdhary, S. Empirical modeling using genetic programming: a survey of issues and approaches. Natural Computing 14, 2 (2015), 303-330.

[36] Das, S., AND Suganthan, P. Differential evolution: A survey of the state-of-the-art. IEEE Transactions on Evolutionary Computation 15, 1 (Feb 2011), 4-31.

[37] Deb, K., Pratap, A., Agarwal, S., And Meyarivan, T. A fast and elitist multiobjective genetic algorithm: Nsga-ii. IEEE Transactions on Evolutionary Computation 6, 2 (Apr 2002), 182-197.

[38] Dorigo, M., Birattari, M., And Stutzle, T. Ant colony optimization. IEEE Computational Intelligence Magazine 1, 4 (Nov 2006), 28-39.

[39] Dorigo, M., AND Di CARO, G. New ideas in optimization. McGrawHill Ltd., UK, Maidenhead, UK, England, 1999, ch. The Ant Colony Optimization Meta-heuristic, pp. 11-32.

[40] Dorigo, M., ANd StÜtzle, T. Ant Colony Optimization. Bradford Company, Scituate, MA, USA, 2004. 
[41] Eberhart, R., AND Kennedy, J. A new optimizer using particle swarm theory. In Proceedings of the Sixth International Symposium on Micro Machine and Human Science (MHS'95) (Oct 1995), pp. 39-43.

[42] EibEn, A. E., AND SMITH, J. E. Introduction to Evolutionary Computing. Springer Berlin Heidelberg, Berlin, Heidelberg, 2015.

[43] Elbeltagi, E., Hegazy, T., And Grierson, D. Comparison among five evolutionary-based optimization algorithms. Adv. Eng. Inform. 19, 1 (2005), 43-53.

[44] Engelbrecht, A. P. Computational Intelligence: An Introduction, 2nd ed. Wiley Publishing, 2007.

[45] Espinosa, J., VANDeWAlle, J., AND Wertz, V. Constructing fuzzy models from input-output data. In Fuzzy Logic, Identification and Predictive Control, Advances in Industrial Control. Springer London, 2005, pp. 21-58.

[46] FenG, G. A survey on analysis and design of model-based fuzzy control systems. IEEE Transactions on Fuzzy Systems 14, 5 (Oct 2006), 676-697.

[47] Fleming, P., AND Purshouse, R. Evolutionary algorithms in control systems engineering: a survey. Control Engineering Practice 10, 11 (2002), 1223 - 1241.

[48] FOGEL, L. J. Intelligence Through Simulated Evolution: Forty Years of Evolutionary Programming. John Wiley \& Sons, Inc., New York, NY, USA, 1999.

[49] Francesco, M. D., Anastasi, G., Conti, M., Das, S. K., AND NERI, V. Reliability and energy-efficiency in ieee 802.15.4/zigbee sensor networks: An adaptive and cross-layer approach. IEEE Journal on Selected Areas in Communications 29, 8 (September 2011), 1508-1524. 
[50] Fulcher, J. Computational Intelligence: An Introduction. Springer Berlin Heidelberg, Berlin, Heidelberg, 2008, pp. 3-78.

[51] G. KO, J., H. CHO, Y., AND KIM, H. Performance evaluation of ieee 802.15.4 mac with different backoff ranges in wireless sensor networks. In 2006 10th IEEE Singapore International Conference on Communication Systems (Oct 2006), pp. 1-5.

[52] Garcia-Galan, S., Prado, R., And Munoz Exposito, J. Swarm fuzzy systems: Knowledge acquisition in fuzzy systems and its applications in grid computing. IEEE Transactions on Knowledge and Data Engineering 26, 7 (July 2014), 1791-1804.

[53] Golmie, N., CYPher, D., And Rebala, O. Performance analysis of low rate wireless technologies for medical applications. Computer Communications 28, 10 (2005), 1266-1275.

[54] Golmie, N., CYPher, D., And Rebala, O. Performance analysis of low rate wireless technologies for medical applications. Computer Communications 28, 10 (2005), 1266 - 1275. Performance issues of Wireless LANs, PANs and ad hoc networks.

[55] GRÄNING, L., JiN, Y., AND SENDHOFF, B. Efficient evolutionary optimization using individual-based evolution control and neural networks: A comparative study. In European Symposium on Artificial Neural Networks (2005), pp. 273-278.

[56] GRÄNING, L., JIN, Y., AND SENDHOFF, B. Individual-based Management of Meta-models for Evolutionary Optimization with Application to Three-Dimensional Blade Optimization. Springer Berlin Heidelberg, Berlin, Heidelberg, 2007, pp. 225-250.

[57] Guo, Y., Li, W., Mileham, A., AND Owen, G. Applications of particle swarm optimisation in integrated process planning and schedul- 
ing. Robotics and Computer-Integrated Manufacturing 25, 2 (2009), 280 288.

[58] HA, J. Y., Kim, T. H., PARK, H. S., CHOI, S., AND KWON, W.-H. An enhanced CSMA-CA algorithm for IEEE 802.15.4 LR-WPANs. IEEE Communications Letters 11, 5 (May 2007), 461-463.

[59] Hachicha, N., Jarboui, B., AND SiArry, P. A fuzzy logic control using a differential evolution algorithm aimed at modelling the financial market dynamics. Information Sciences 181, 1 (2011), 79 - 91.

[60] Han, H., Su, C.-Y., And Stepanenko, Y. Adaptive control of a class of nonlinear systems with nonlinearly parameterized fuzzy approximators. IEEE Transactions on Fuzzy Systems 9, 2 (Apr 2001), 315-323.

[61] Hanson, M. A., Powell JR., H. C., Barth, A. T., Ringgenberg, K., CAlhoun, B. H., AYlor, J. H., AND LACH, J. Body area sensor networks: Challenges and opportunities. Computer 42, 1 (Jan. 2009), $58-65$.

[62] Holland, J. H. Adaptation in Natural and Artificial Systems: An Introductory Analysis with Applications to Biology, Control and Artificial Intelligence. MIT Press, Cambridge, MA, USA, 1992.

[63] Holland, J. H., Booker, L. B., Colombetti, M., Dorigo, M., GoldberG, D. E., Forrest, S., RiOlo, R. L., SMith, R. E., LANZI, P. L., STOLZMANN, W., AND Wilson, S. W. What Is a Learning Classifier System? Springer Berlin Heidelberg, Berlin, Heidelberg, 2000, pp. 3-32.

[64] Huang, Y. K., PAng, A. C., AND Hung, H. N. An adaptive gts allocation scheme for ieee 802.15.4. IEEE Transactions on Parallel and Distributed Systems 19, 5 (May 2008), 641-651. 
[65] Hurel, J., Mandow, A., AND Garcia-Cerezo, A. Tuning a fuzzy controller by particle swarm optimization for an active suspension system. In 38th Annual Conference on IEEE Industrial Electronics Society (IECON'12) (Oct 2012), pp. 2524-2529.

[66] HusBand, P. Distributed coevolutionary genetic algorithms for multicriteria and multi-constraint optimisation. Springer Berlin Heidelberg, Berlin, Heidelberg, 1994.

[67] HÜSKen, M., Jin, Y., AND SendHOFF, B. Structure optimization of neural networks for evolutionary design optimization. Soft Computing 9, 1 (Jan 2005), 21-28.

[68] Hussein, A. A., AND Ali, M. H. Fuzzy logic controlled variable resistor for suppressing gic in transformers. IET Generation, Transmission Distribution 11, 6 (2017), 1494-1501.

[69] IbÁÑ̃zZ, O., CORdón, O., AND DAMAS, S. A cooperative coevolutionary approach dealing with the skull-face overlay uncertainty in forensic identification by craniofacial superimposition. Soft Computing 16, 5 (2012), 797-808.

[70] ICHIHASHI, H., AND TOKUNAGA, M. Neuro-fuzzy optimal control of backing up a trailer truck. In IEEE International Conference on Neural Networks (1993), pp. 306-311 vol.1.

[71] IoAnnou, P. A., AND Sun, J. Robust Adaptive Control. Prentice-Hall, Inc., Upper Saddle River, NJ, USA, 1995.

[72] Istepanian, R. S. H., Jovanov, E., AND ZhanG, Y. T. Guest editorial introduction to the special section on $\mathrm{m}$-health: Beyond seamless mobility and global wireless health-care connectivity. IEEE Transactions on Information Technology in Biomedicine 8 (2004). 
[73] Jin, R., CHEN, W., AND Simpson, T. Comparative studies of metamodelling techniques under multiple modelling criteria. Structural and Multidisciplinary Optimization 23, 1 (2001), 1-13.

[74] JIN, Y. A comprehensive survey of fitness approximation in evolutionary computation. Soft Computing 9, 1 (2005), 3-12.

[75] JIN, Y. Surrogate-assisted evolutionary computation: Recent advances and future challenges. Swarm and Evolutionary Computation 1, 2 (2011), $61-70$.

[76] JiN, Y., Olhofer, M., AND SENDHOFF, B. A framework for evolutionary optimization with approximate fitness functions. IEEE Transactions on Evolutionary Computation 6, 5 (Oct 2002), 481-494.

[77] Jin, Y., OlHOFER, M., AND SENDHOFF, B. A framework for evolutionary optimization with approximate fitness functions. Trans. Evol. Comp 6, 5 (Oct. 2002), 481-494.

[78] Johansen, T. A., Shorten, R., And Murray-Smith, R. On the interpretation and identification of dynamic takagi-sugeno fuzzy models. IEEE Transactions on Fuzzy Systems 8, 3 (Jun 2000), 297-313.

[79] Jovanov, E., Milenkovic, A., Otto, C., And de Groen, P. C. A wireless body area network of intelligent motion sensors for computer assisted physical rehabilitation. Journal of NeuroEngineering and Rehabilitation 2, 1 (2005), 6.

[80] KaChitvichyanukul, V. Comparison of three evolutionary algorithms: Ga, pso, and de. Industrial Engineering and Management Systems 11, 3 (2012), 215-223.

[81] Kennedy, J., AND Eberhart, R. Particle swarm optimization. In Neural Networks, 1995. Proceedings., IEEE International Conference on (Nov 1995), vol. 4, pp. 1942-1948 vol.4. 
[82] Kennedy, J., Eberhart, R. C., AND ShI, Y. Swarm Intelligence. The Morgan Kaufmann Series in Artificial Intelligence. Morgan Kaufmann, San Francisco, 2001.

[83] Khanafer, M., Guennoun, M., And Mouftah, H. A survey of beacon-enabled ieee 802.15.4 mac protocols in wireless sensor networks. IEEE Communications Surveys Tutorials 16, 2 (Second 2014), $856-876$.

[84] Kim, J., MoON, Y., AND Zeigler, B. Designing fuzzy net controllers using genetic algorithms. IEEE Control Systems 15, 3 (Jun 1995), 66-72.

[85] King, P., And Mamdani, E. The application of fuzzy control systems to industrial processes. Automatica 13, 3 (1977), 235 - 242.

[86] KoubÂA, A., Alves, M., Tovar, E., And Cunha, A. An implicit gts allocation mechanism in ieee 802.15.4 for time-sensitive wireless sensor networks: Theory and practice. Real-Time Systems 39, 1-3 (2008), 169-204.

[87] KozA, J. R. Genetic Programming: On the Programming of Computers by Means of Natural Selection. MIT Press, Cambridge, MA, USA, 1992.

[88] KozA, J. R. Introduction to genetic programming: Tutorial. In Proceedings of the 10th Annual Conference Companion on Genetic and Evolutionary Computation (New York, NY, USA, 2008), GECCO '08, ACM, pp. 2299-2338.

[89] KRAMES, E. Implantable devices for pain control: spinal cord stimulation and intrathecal therapies. Best Practice \& Research Clinical Anaesthesiology 16, 4 (2002), 619 - 649.

[90] Krstic, M., Kokotovic, P. V., And Kanellakopoulos, I. Nonlinear and Adaptive Control Design, 1st ed. John Wiley \& Sons, Inc., New York, NY, USA, 1995. 
[91] Latré, B., Braem, B., Moerman, I., Blondia, C., and DeMEESTER, P. A survey on wireless body area networks. Wireless Networks 17, 1 (2011), 1-18.

[92] LI, X., AND YAO, X. Tackling high dimensional nonseparable optimization problems by cooperatively coevolving particle swarms. In 2009 IEEE Congress on Evolutionary Computation (May 2009), pp. 15461553.

[93] LI, X., AND YAO, X. Cooperatively coevolving particle swarms for large scale optimization. IEEE Transactions on Evolutionary Computation 16, 2 (April 2012), 210-224.

[94] LIANG, Q., AND WANG, L. Event detection in wireless sensor networks using fuzzy logic system. In IEEE International Conference on Computational Intelligence for Homeland Security and Personal Safety (March 2005), pp. 52-55.

[95] LiN, S.-C., AND CHEN, Y.-Y. Design of self-learning fuzzy sliding mode controllers based on genetic algorithms. Fuzzy Sets and Systems 86, 2 (1997), $139-153$.

[96] Liu, B. D., Chen, C. Y., AND TsaO, J. Y. Design of adaptive fuzzy logic controller based on linguistic-hedge concepts and genetic algorithms. IEEE Transactions on Systems, Man, and Cybernetics, Part B: Cybernetics 31, 1 (Feb 2001), 32-53.

[97] Loshchilov, I., Schoenauer, M., And Sebag, M. Intensive surrogate model exploitation in self-adaptive surrogate-assisted cmaes (saacm-es). In Proceedings of the 15th Annual Conference on Genetic and Evolutionary Computation (2013), GECCO '13, ACM, pp. 439-446.

[98] Lu, G., KRishnamachari, B., And Raghavendra, C. S. An adaptive energy-efficient and low-latency mac for data gathering in 
wireless sensor networks. In 18th International Parallel and Distributed Processing Symposium, 2004. Proceedings. (April 2004), pp. 224-.

[99] Martinez-Soto, R., Castillo, O., Aguilar, L., and Melin, P. Fuzzy logic controllers optimization using genetic algorithms and particle swarm optimization. In Advances in Soft Computing, G. Sidorov, A. Hernndez Aguirre, and C. Reyes Garca, Eds., vol. 6438 of Lecture Notes in Computer Science. Springer Berlin Heidelberg, 2010, pp. $475-486$.

[100] Mendel, J. M. Uncertain Rule-Based Fuzzy Logic Systems: Introduction and New Directions, 1st ed. Prentice Hall, 2001.

[101] Mhemed, R., Aslam, N., Phillips, W., and Comeau, F. An energy efficient fuzzy logic cluster formation protocol in wireless sensor networks. Procedia Computer Science 10 (2012), 255 - 262.

[102] Milla, F., Saez, D., Cortes, C., And Cipriano, A. Bus-stop control strategies based on fuzzy rules for the operation of a public transport system. IEEE Transactions on Intelligent Transportation Systems 13, 3 (Sept 2012), 1394-1403.

[103] Mitchell, T. M. Machine Learning, 1 ed. McGraw-Hill, Inc., New York, NY, USA, 1997.

[104] MitRA, S., AND HAYASHI, Y. Neuro-fuzzy rule generation: survey in soft computing framework. IEEE Transactions on Neural Networks 11, 3 (May 2000), 748-768.

[105] MotA, F., AND Gomide, F. Fuzzy clustering in fitness estimation models for genetic algorithms and applications. In 2006 IEEE International Conference on Fuzzy Systems (2006), pp. 1388-1395.

[106] Moulton, B., Chen, J., Croucher, G., Lal, S., LaWrence, E., MAHENDRAN, L., AND VARIS, A. Ambulatory health monitoring 
and remote sensing systems to be used by outpatients and elders at home: User-related design considerations. In 2009 11th International Conference on e-Health Networking, Applications and Services (Healthcom) (Dec 2009), pp. 48-53.

[107] MouzehKesh, N., Zia, T., SHAfigh, S., AND ZHeNG, L. D²MAC: Dynamic delayed medium access control (MAC) protocol with fuzzy technique for wireless body area networks. In IEEE International Conference on Body Sensor Networks (BSN) (May 2013), pp. 1-6.

[108] Mouzehkesh, N., Zia, T., Shafigh, S., And Zheng, L. Dynamic backoff scheduling of low data rate applications in wireless body area networks. Wireless Networks 21, 8 (Nov. 2015), 2571-2592.

[109] Movassaghi, S., Abolhasan, M., Lipman, J., Smith, D., AND JAMALIPOUR, A. Wireless body area networks: A survey. IEEE Communications Surveys Tutorials 16, 3 (2014), 1658-1686.

[110] NA, C., YANG, Y., AND Mishra, A. An optimal gts scheduling algorithm for time-sensitive transactions in ieee 802.15.4 networks. Computer Networks 52, 13 (2008), 2543 - 2557.

[111] NABI, A., AND SINGH, N. A. Particle swarm optimization of fuzzy logic controller for voltage sag improvement. In 2016 3rd International Conference on Advanced Computing and Communication Systems (ICACCS) (Jan 2016), vol. 01, pp. 1-5.

[112] Natsheh, E., AND Buragga, K. A. Comparison between conventional and fuzzy logic pid controllers for controlling dc motors. International Journal of Computer Science 7, 5 (Sep 2010), 128-134.

[113] Nekooei, S. M., Chen, G., And Rayudu, R. K. A fuzzy logic based cross-layer mechanism for medium access control in wban. In IEEE 26th Annual International Symposium on Personal, Indoor, and Mobile Radio Communications (PIMRC) (Aug 2015), pp. 1094-1099. 
[114] Nekooei, S. M., Chen, G., And Rayudu, R. K. Evolutionary design of fuzzy logic controllers for medium access control in wban. In IEEE Congress on Evolutionary Computation (IEEE CEC) (July 2016), pp. 2821-2828.

[115] NeKooei, S. M., Chen, G., And Rayudu, R. K. Automatic design of fuzzy logic controllers for medium access control in wireless body area networks an evolutionary approach. Applied Soft Computing 56 (2017), $245-261$.

[116] Nekooei, S. M., Chen, G., AND Rayudu, R. K. Cooperative design of two level fuzzy logic controllers for medium access control in wireless body area networks. In International Conference on Simulated Evolution and Learning (SEAL2017) (November 2017).

[117] NekooeI, S. M., AND MAnZuri-Shalmani, M. T. Location finding in wireless sensor network based on soft computing methods. In 2011 International Conference on Control, Automation and Systems Engineering (CASE) (July 2011), pp. 1-5.

[118] OH, S.-K., Kim, W.-D., AND Pedrycz, W. Design of optimized cascade fuzzy controller based on differential evolution: Simulation studies and practical insights. Engineering Applications of Artificial Intelligence 25, 3 (2012), $520-532$.

[119] Otal, B., Alonso, L., AND Verikoukis, C. Highly reliable energysaving mac for wireless body sensor networks in healthcare systems. IEEE Journal on Selected Areas in Communications 27, 4 (2009), 553-565.

[120] Otal, B., Alonso, L., AND Verikoukis, C. Design and analysis of an energy-saving distributed MAC mechanism for wireless body sensor networks. EURASIP Journal on Wireless Communications and Networking 27, 4 (2010), 1-13. 
[121] PARK, S., AND JAYARAMAN, S. Enhancing the quality of life through wearable technology. IEEE Engineering in Medicine and Biology Magazine 22 (2003).

[122] Parno, M. D., Hemker, T., And Fowler, K. R. Applicability of surrogates to improve efficiency of particle swarm optimization for simulation-based problems. Engineering Optimization 44, 5 (2012), 521-535.

[123] PeDRyCZ, W. An identification algorithm in fuzzy relational systems. Fuzzy Sets and Systems 13, 2 (1984), 153 - 167.

[124] Pishkenari, H. N., Mahboobi, S., And Alasty, A. Optimum synthesis of fuzzy logic controller for trajectory tracking by differential evolution. Scientia Iranica 18, 2 (2011), 261 - 267.

[125] Poli, R., Kennedy, J., And Blackwell, T. Particle swarm optimization. Swarm Intelligence 1, 1 (2007), 33-57.

[126] PotTer, M. A., AND Jong, K. A. D. A cooperative coevolutionary approach to function optimization. In Proceedings of the International Conference on Evolutionary Computation. The Third Conference on Parallel Problem Solving from Nature: Parallel Problem Solving from Nature (London, UK, UK, 1994), PPSN III, Springer-Verlag, pp. 249-257.

[127] Praveen, C., And Duvigneau, R. Low cost PSO using metamodels and inexact pre-evaluation: Application to aerodynamic shape design. Computer Methods in Applied Mechanics and Engineering 198, 912 (2009), 1087 - 1096.

[128] Price, K., StORn, R. M., And LAMPInen, J. A. Differential Evolution: A Practical Approach to Global Optimization (Natural Computing Series). Springer-Verlag New York, Inc., Secaucus, NJ, USA, 2005. 
[129] Regis, R. G. Particle swarm with radial basis function surrogates for expensive black-box optimization. Journal of Computational Science 5, 1 (2014), $12-23$.

[130] Reusens, E., Joseph, W., Vermeeren, G., Kurup, D., And MARTENS, L. Real human body measurements, model, and simulations of a $2.45 \mathrm{ghz}$ wireless body area network communication channel. In 2008 5th International Summer School and Symposium on Medical Devices and Biosensors (June 2008), pp. 149-152.

[131] Reusens, E., Joseph, W., Vermeeren, G., Kurup, D., AND MARTENS, L. Real human body measurements, model, and simulations of a $2.45 \mathrm{ghz}$ wireless body area network communication channel. In ISSS-MDBS 2008. 5th International Summer School and Symposium on Medical Devices and Biosensors (June 2008), pp. 149-152.

[132] Reusens, E., Joseph, W., Vermeeren, G., And Martens, L. On-Body Measurements and Characterization of Wireless Communication Channel for Arm and Torso of Human. Springer Berlin Heidelberg, Berlin, Heidelberg, 2007, pp. 264-269.

[133] RoberTS, M. E., AND Claridge, E. Cooperative Coevolution of Image Feature Construction and Object Detection. Springer Berlin Heidelberg, 2004, pp. 902-911.

[134] SHI, Y., AND EBERHART, R. A modified particle swarm optimizer. In Evolutionary Computation Proceedings (May 1998), pp. 69-73.

[135] Shrestha, B., Hossain, E., Camorlinga, S., Krishnamoorthy, R., AND NIYATO, D. An optimization-based gts allocation scheme for ieee 802.15.4 mac with application to wireless body-area sensor networks. In 2010 IEEE International Conference on Communications (ICC) (2010), pp. 1-6. 
[136] SHU, H., AND Liang, Q. Spectrum efficient coding scheme for correlated non-binary sources in wireless sensor networks. In IEEE Military Communications Conference (Oct 2005), pp. 911-917 Vol. 2.

[137] SHU, H., LiAnG, Q., AND GAO, J. Wireless sensor network lifetime analysis using interval type-2 fuzzy logic systems. IEEE Transactions on Fuzzy Systems 16, 2 (Apr. 2008), 416-427.

[138] Shuai, L., AND WeI, S. Design of Fuzzy Logic Controller Based on Differential Evolution Algorithm. Springer Berlin Heidelberg, Berlin, Heidelberg, 2014, pp. 18-25.

[139] Smith, D., Miniutti, D., And Hanlen, L. Characterization of the body-area propagation channel for monitoring a subject sleeping. IEEE Transactions on Antennas and Propagation 59, 11 (2011), 4388-4392.

[140] Smith, D. B., Miniutti, D., AND Hanlen, L. W. Characterization of the body-area propagation channel for monitoring a subject sleeping. IEEE Transactions on Antennas and Propagation 59, 11 (Nov 2011), 4388-4392.

[141] STANFORD, V. Using pervasive computing to deliver elder care. IEEE Pervasive Computing 1, 1 (Jan. 2002), 10-13.

[142] Stoica, A., Klimeck, G., Salazar-Lazaro, C., Keymeulen, D., AND THAKOOR, A. Evolutionary design of electronic devices and circuits. In Proceedings of the 1999 Congress on Evolutionary Computation (CEC) (1999), vol. 2, p. 1278 Vol. 2.

[143] STONIER, R. J., AND YOUNG, N. Co-evolutionary learning and hierarchical fuzzy control for the inverted pendulum. In The 2003 Congress on Evolutionary Computation (CEC'3) (Dec 2003), vol. 1, pp. 467-473 Vol.1. 
[144] Storn, R., AND PRice, K. Differential evolution - a simple and efficient heuristic for global optimization over continuous spaces. Journal of Global Optimization 11, 4 (1997), 341-359.

[145] Sugeno, M. On stability of fuzzy systems expressed by fuzzy rules with singleton consequents. IEEE Transactions on Fuzzy Systems 7, 2 (Apr 1999), 201-224.

[146] TAKagi, T., AND Sugeno, M. Fuzzy identification of systems and its applications to modeling and control. IEEE Transactions on Systems, Man, and Cybernetics SMC-15, 1 (Jan 1985), 116-132.

[147] Taparugssanagorn, A., Rabbachin, A., HämÄläinen, M., SALORANTA, J., IINATTI, J., AND MEMBER, S. A review of channel modelling for wireless body area network in wireless medical communications. In The 11th International Symposium on Wireless Personal Multimedia Communications (2008), WPMC '8.

[148] Tidjani, F. S., Hamadi, A., Chandra, A., Pillay, P., And NDTOUNGOU, A. Optimization of standalone microgrid considering active damping technique and smart power management using fuzzy logic supervisor. IEEE Transactions on Smart Grid 8, 1 (2017), 475-484.

[149] Tuan, H. D., Apkarian, P., Narikiyo, T., And Yamamoto, Y. Parameterized linear matrix inequality techniques in fuzzy control system design. IEEE Transactions on Fuzzy Systems 9, 2 (Apr 2001), 324-332.

[150] TušAR, T., AND FILIPIČ, B. Differential Evolution versus Genetic Algorithms in Multiobjective Optimization. Springer Berlin Heidelberg, Berlin, Heidelberg, 2007, pp. 257-271.

[151] Ullah, S., Higgins, H., Braem, B., Latre, B., Blondia, C., Moerman, I., SAleEM, S., RAHMAN, Z., AND KWAK, K. S. A 
comprehensive survey of wireless body area networks. Journal of Medical Systems 36, 3 (2012), 1065-1094.

[152] Ullah, S., Shen, B., Islam, S. M. R., KHAN, P., SAleEM, S., AND KWAK, K. S. A study of mac protocols for wbans. Sensors (Basel, Switzerland) 10, 1 (2010), 128-145.

[153] UN. World Population Prospects The 2015 Revision: Key Findings and Advance Tables. United Nations, 2015.

[154] UTKIN, V. I. Sliding Modes in Control and Optimization. Springer Berlin Heidelberg, Berlin, Heidelberg, 1992.

[155] VAN DAM, T., AND LANGENDOEN, K. An adaptive energy-efficient mac protocol for wireless sensor networks. In Proceedings of the 1st International Conference on Embedded Networked Sensor Systems (2003), SenSys '03, ACM, pp. 171-180.

[156] VAn Den Bergh, F., AND Engelbrecht, A. P. A cooperative approach to particle swarm optimization. IEEE Transactions on Evolutionary Computation 8, 3 (2004), 225-239.

[157] Vesterstrom, J., AND Thomsen, R. A comparative study of differential evolution, particle swarm optimization, and evolutionary algorithms on numerical benchmark problems. In Evolutionary Computation, 2004. CEC2004. Congress on (June 2004), vol. 2, pp. 1980-1987 Vol.2.

[158] WANG, D., TAN, D., AND LIU, L. Particle swarm optimization algorithm: an overview. Soft Computing (2017), 1-22.

[159] Wang, L. X. Adaptive Fuzzy Systems and Control: Design and Stability Analysis. Prentice-Hall, Inc., 1994. 
[160] WANG, L. X., AND Mendel, J. M. Fuzzy basis functions, universal approximation, and orthogonal least-squares learning. Trans. Neur. Netw. 3, 5 (Sept. 1992), 807-814.

[161] Wiegand, R. P., Liles, W. C., AND JonG, K. A. D. An empirical analysis of collaboration methods in cooperative coevolutionary algorithms. In Proceedings of the 3rd Annual Conference on Genetic and Evolutionary Computation (2001), GECCO'01, pp. 1235-1242.

[162] Winters, J. M., WANG, Y., AND Winters, J. M. Wearable sensors and telerehabilitation. IEEE Engineering in Medicine and Biology Magazine 22, 3 (May 2003), 56-65.

[163] WONG, C. M., AND LEE, B. H. An improvement of slotted CSMA/CA algorithm in IEEE 802.15.4 medium access layer. Wirel. Pers. Commun. 63, 4 (2012), 807-822.

[164] XiA, F., HAO, R., CAO, Y., AND XUE, L. A survey of adaptive and real-time protocols based on ieee 802.15.4. International Journal of Distributed Sensor Networks 7, 1 (2011), 212737.

[165] XIA, F., AND RAHIM, A. IEEE 802.15.4 Based Adaptive MAC Protocols. Springer, Berlin, Heidelberg, 2015, pp. 53-68.

[166] XiaO, J., XiaO, J. Z., XI, N., Tummala, R. L., AND Mukherjee, R. Fuzzy controller for wall-climbing microrobots. IEEE Transactions on Fuzzy Systems 12, 4 (Aug 2004), 466-480.

[167] Xue, B., ZHANG, M., AND BROWNe, W. Particle swarm optimization for feature selection in classification: A multi-objective approach. IEEE Transactions on Cybernetics 43, 6 (Dec 2013), 1656-1671.

[168] YAger, R. R., AND FileV, D. P. Essentials of Fuzzy Modeling and Control. Wiley-Interscience, New York, NY, USA, 1994. 
[169] YANG, D., XU, Y., WANG, H., ZHENG, T., ZHANG, H., ZHANG, H., AND GIDLUND, M. Assignment of segmented slots enabling reliable real-time transmission in industrial wireless sensor networks. IEEE Transactions on Industrial Electronics 62, 6 (June 2015), 3966-3977.

[170] YanG, S. X., LI, H., MenG, M. Q. H., And LiU, P. X. An embedded fuzzy controller for a behavior-based mobile robot with guaranteed performance. IEEE Transactions on Fuzzy Systems 12, 4 (Aug 2004), 436-446.

[171] Ye, W., HEIDEMANN, J., AND EsTRIN, D. An energy-efficient mac protocol for wireless sensor networks. In Twenty-First Annual Joint Conference of the IEEE Computer and Communications Societies (2002), vol. 3, pp. 1567-1576 vol.3.

[172] Yin, H., Zhou, W., Li, M., MA, C., AND ZhaO, C. An adaptive fuzzy logic-based energy management strategy on battery/ultracapacitor hybrid electric vehicles. IEEE Transactions on Transportation Electrification 2, 3 (Sept 2016), 300-311.

[173] Yun, S., LeE, J., ChunG, W., Kim, E., AND Kim, S. A soft computing approach to localization in wireless sensor networks. Expert Systems with Applications 36, 4 (2009), 7552 - 7561.

[174] ZADEH, L. Fuzzy sets. Information and Control 8, 3 (1965), 338 - 353.

[175] ZADEH, L. A. Outline of a new approach to the analysis of complex systems and decision processes. IEEE Transactions on Systems, Man, and Cybernetics SMC-3, 1 (Jan 1973), 28-44.

[176] ZHAO, L., AND LiANG, Q. Fuzzy deployment for wireless sensor networks. In IEEE International Conference on Computational Intelligence for Homeland Security and Personal Safety (March 2005), pp. 79-83. 
[177] ZhOU, J., GuO, A., XU, J., AND SU, S. An optimal fuzzy control medium access in wireless body area networks. Neurocomputing 142 (2014), 107 - 114. \{SI\} Computational Intelligence Techniques for New Product Development.

[178] ZHOU, S. M., AND GAN, J. Q. Low-level interpretability and highlevel interpretability: A unified view of data-driven interpretable fuzzy system modelling. Fuzzy Sets Syst. 159, 23 (Dec. 2008), 30913131.

[179] Zinober, A. S. I. Variable Structure and Lyapunov Control. Springer Berlin Heidelberg, Berlin, Heidelberg, 1994.

[180] Zitzler, E., LaumanNS, M., AND ThIELE, L. Spea2: Improving the strength pareto evolutionary algorithm for multiobjective optimization. In Evolutionary Methods for Design, Optimisation, and Control (2002), CIMNE, Barcelona, Spain, pp. 95-100. 


\section{Appendix A}

\section{High performance Computation}

NeSI provides High Performance Computing (HPC) platforms in New Zealand. The specifications of platforms are summarised in Table A.1.

Table A.1: The specifications of High Performance Computing (HPC) platforms

\begin{tabular}{|l|l|l|}
\hline Platform & Hardware & Operating Environment \\
\hline \multirow{5}{*}{ P575/POWER6 } & - 108 nodes each with 32 cores & - AIX \\
FitzRoy & (3,456 4.7 GHz processor cores) & - GPFS high performance parallel \\
& with each node having either 64 or & file system \\
& RAM in total. & - LoadLeveler workload scheduler \\
& - DDR InfiniBand interconnect & and TSM-SM Hierarchical Storage \\
& between nodes & Management (HSM) \\
\hline \multirow{5}{*}{ Intel cluster } & - 6,000+ cores of Intel based x86 & \\
& processors (IvyBridge/SandyBridge & - Linux \\
& /Westmere) operating at 2.7-2.8 GHz & - GPFS high performance parallel \\
& with approximately 8 GB RAM per core & file system \\
& K20X models) & - SLURM workload scheduler \\
& - 4 Intel Xeon Phi devices & and TSM-SM Hierarchical Storage \\
& (Intel Phi 5110P) QDR Infiniband & Management (HSM) \\
& interconnect & \\
\hline
\end{tabular}

